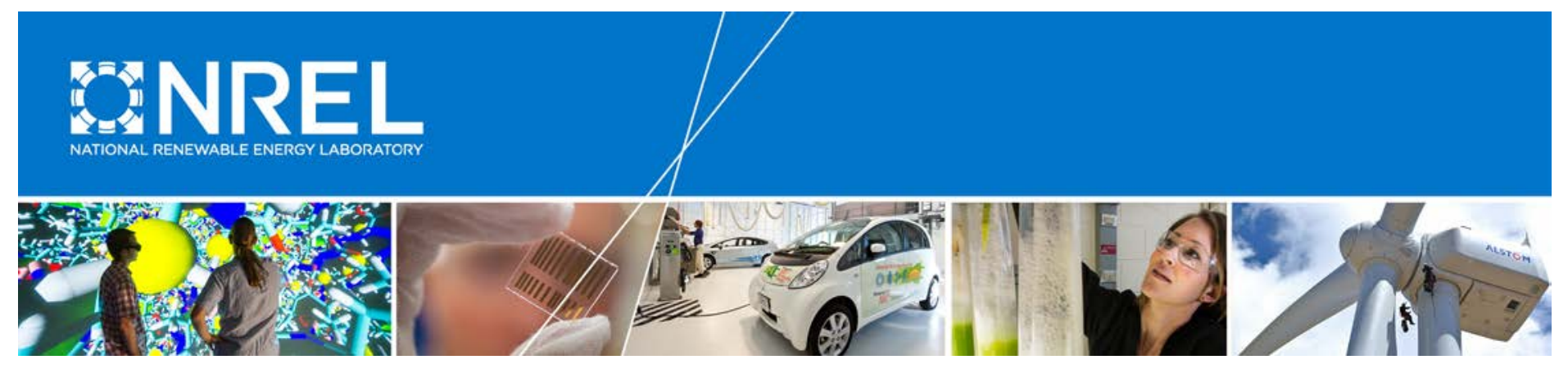

\title{
Eastern Renewable Generation Integration Study
}

Aaron Bloom ${ }^{1}$, Aaron Townsend ${ }^{1}$, David Palchak ${ }^{1}$, Joshua Novacheck ${ }^{1}$, Jack King ${ }^{2}$, Clayton Barrows ${ }^{1}$, Eduardo Ibanez $^{3}$, Matthew O'Connell ${ }^{1}$, Gary Jordan ${ }^{3}$, Billy Roberts ${ }^{1}$, Caroline Draxl ${ }^{1}$, and Kenny Gruchalla ${ }^{1}$

${ }^{1}$ National Renewable Energy Laboratory ${ }^{2} \operatorname{RePPAE} L L C$

${ }^{3} G E$

Executive Summary

NREL is a national laboratory of the U.S. Department of Energy Office of Energy Efficiency \& Renewable Energy Operated by the Alliance for Sustainable Energy, LLC

This report is available at no cost from the National Renewable Energy Laboratory (NREL) at www.nrel.gov/publications.

Technical Report

NREL/TP-6A20-64472

August 2016

Contract No. DE-AC36-08G028308 


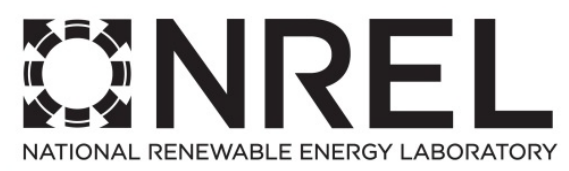

\section{Eastern Renewable Generation Integration Study}

Aaron Bloom ${ }^{1}$, Aaron Townsend ${ }^{1}$, David Palchak $^{1}$, Joshua Novacheck ${ }^{1}$, Jack King ${ }^{2}$, Clayton Barrows ${ }^{1}$, Eduardo Ibanez ${ }^{3}$, Matthew O'Connell ${ }^{1}$, Gary Jordan ${ }^{3}$, Billy Roberts ${ }^{1}$, Caroline Draxl ${ }^{1}$, and Kenny Gruchalla ${ }^{1}$

${ }^{1}$ National Renewable Energy Laboratory

${ }^{2} \operatorname{RePPAE}$ LLC

${ }^{3} \mathrm{GE}$

Prepared under Task No(s). WE14.8A01, SS13.3011, OE10.4010

Executive Summary

NREL is a national laboratory of the U.S. Department of Energy Office of Energy Efficiency \& Renewable Energy Operated by the Alliance for Sustainable Energy, LLC

This report is available at no cost from the National Renewable Energy Laboratory (NREL) at www.nrel.gov/publications.

National Renewable Energy Laboratory 15013 Denver West Parkway Golden, CO 80401

303-275-3000 • www.nrel.gov
Technical Report

NREL/TP-6A20-64472

August 2016

Contract No. DE-AC36-08GO28308 


\section{NOTICE}

This report was prepared as an account of work sponsored by an agency of the United States government. Neither the United States government nor any agency thereof, nor any of their employees, makes any warranty, express or implied, or assumes any legal liability or responsibility for the accuracy, completeness, or usefulness of any information, apparatus, product, or process disclosed, or represents that its use would not infringe privately owned rights. Reference herein to any specific commercial product, process, or service by trade name, trademark, manufacturer, or otherwise does not necessarily constitute or imply its endorsement, recommendation, or favoring by the United States government or any agency thereof. The views and opinions of authors expressed herein do not necessarily state or reflect those of the United States government or any agency thereof.

This report is available at no cost from the National Renewable Energy Laboratory (NREL) at www.nrel.gov/publications.

Available electronically at SciTech Connect http:/www.osti.gov/scitech

Available for a processing fee to U.S. Department of Energy and its contractors, in paper, from:

U.S. Department of Energy

Office of Scientific and Technical Information

P.O. Box 62

Oak Ridge, TN 37831-0062

OSTI http://www.osti.gov

Phone: 865.576.8401

Fax: 865.576.5728

Email: reports@osti.gov

Available for sale to the public, in paper, from:

U.S. Department of Commerce

National Technical Information Service

5301 Shawnee Road

Alexandria, VA 22312

NTIS http://www.ntis.gov

Phone: 800.553 .6847 or 703.605 .6000

Fax: 703.605.6900

Email: orders@ntis.gov 


\section{Acknowledgments}

The authors appreciate the sponsorship of the Department of Energy's (DOE) Office of Energy Efficiency and Renewable Energy Wind Program and Solar Energy Technologies Office as well as the Office of Electricity Delivery \& Energy Reliability's National Electricity Delivery Division, who made this work possible. Special thanks go to Charlton Clark, Caitlin Callaghan Guohui Yuan, Venkat Banunarayanan whose vision and guidance helped shape this study.

The author team and report benefited throughout from the oversight and guidance of a Technical Review Committee (TRC). Thanks to TRC members:

NextEra Energy

Great River Energy

Florida Power \& Light

Midcontinent Independent System Operator

Southwest Power Pool

Electric Power Research Institute

Wärtsilla

Solar Energy Industry Association

American Wind Energy Association

Oak Ridge National Laboratory

Edison Electric Institute

Manitoba Hydro

DOE

National Rural Electric Cooperative Association

PJM Interconnection

Utility Variable Generation Integration Group

Whiteley BPS Planning Ventures

EnerNex
Mark Ahlstrom

Jared Alholinna

Andrew Arana

Jordan Bakke

Jay Caspary

Erik Ela and Aidan Tuohy

Joseph Ferrari

Katherine Gensler

Michael Goggin

Stanton Hadley

Maryanne Hatch

David Jacobson

Kevin Lynn, Ookie Ma, and Larry Mansueti

Paul McCurley

Ken Schuyler

Charlie Smith

David Whiteley

Tom Mousseau 
A special thanks goes to all of the TRC members that helped us obtain data for our work, especially Stanton Hadley and David Whitely, for helping us coordinate our work and data with the Eastern Interconnection Planning Collaborative.

Finally, we appreciate the support and contributions of many NREL colleagues. A special thanks goes to Kara Clark, Michael Milligan, David Corbus, Paul Denholm, and Greg Brinkman. Important contributions and advice were also provided by Wes Jones, Dave Biagioni, Debra Lew (GE), Trieu Mai, David Mulchay, Kevin Regimbal, Brady Stoll, and Owen Zinaman. We would also like to acknowledge the impact that the Renewable Electricity Future Team had on the development of these advanced visualization tools, especially Anthony Lopez and Trieu Mai. Scott Gossett enlivened the story by providing editorial support. 


\section{List of Acronyms}

\begin{tabular}{|c|c|}
\hline $\mathrm{AC}$ & alternating current \\
\hline $\mathrm{AEO}$ & Annual Energy Outlook \\
\hline $\mathrm{CC}$ & combined cycle generator \\
\hline CEMS & Continuous Emissions Monitoring Systems \\
\hline CT & combustion turbine generator \\
\hline DOE & U.S. Department of Energy \\
\hline EI & Eastern Interconnection \\
\hline EIA & U.S. Energy Information Administration \\
\hline EIPC & Eastern Interconnection Planning Collaborative \\
\hline EMM & Electricity Market Module \\
\hline EPA & U.S. Environmental Protection Agency \\
\hline EPRI & Electric Power Research Institute \\
\hline ERAG & $\begin{array}{l}\text { Eastern Interconnection Reliability Assessment } \\
\text { Group }\end{array}$ \\
\hline ERGIS & Eastern Renewable Generation Integration Study \\
\hline EWITS & Eastern Wind Integration and Transmission Study \\
\hline FERC & Federal Energy Regulatory Commission \\
\hline FRCC & Florida Reliability Coordinating Council \\
\hline GADS & Generator Availability Data System \\
\hline HVDC & High voltage direct current \\
\hline ISO & independent system operator \\
\hline ISO-NE & Independent System Operator of New England \\
\hline MAE & mean absolute error \\
\hline MISO & Midcontinent Independent System Operator \\
\hline NEMS & National Energy Modeling System \\
\hline NERC & North American Electric Reliability Corporation \\
\hline NREL & National Renewable Energy Laboratory \\
\hline NYISO & New York Independent System Operator \\
\hline PJM & PJM Interconnection \\
\hline PV & photovoltaic \\
\hline ReEDS & Regional Energy Deployment System \\
\hline RMSE & root-mean-square error \\
\hline RPS & renewable portfolio standard \\
\hline RT & real-time \\
\hline RTO & regional transmission operator \\
\hline SEPA & Southeastern Power Administration \\
\hline SERC & SERC Reliability Corporation \\
\hline $\mathrm{SOCO}$ & Southern Company \\
\hline SPI & solar power index \\
\hline SPP & Southwest Power Pool \\
\hline SWPA & Southwestern Power Administration \\
\hline $\mathrm{TRC}$ & Technical Review Committee \\
\hline TVA & Tennessee Valley Authority \\
\hline UC\&ED & unit commitment and economic dispatch \\
\hline USACE & U.S. Army Corps of Engineers \\
\hline
\end{tabular}


UTC

VACAR

$\mathrm{VG}$

Western

WRF

WWSIS-2
Coordinated Universal Time

Virginia-Carolinas sub-region of SERC

variable generation

Western Area Power Administration

Weather Research and Forecasting

Western Wind and Solar Integration Study Phase II 


\section{Table of Contents}

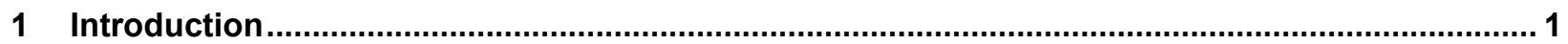

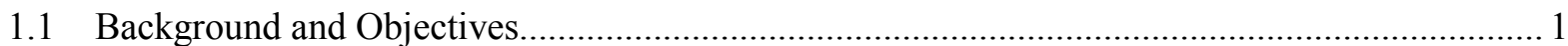

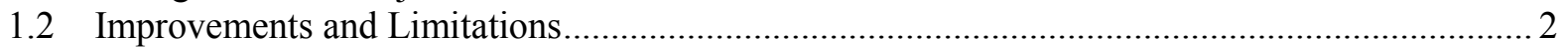

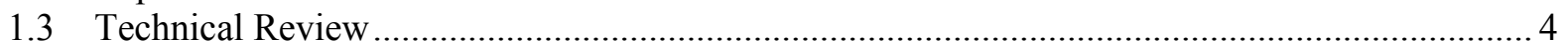

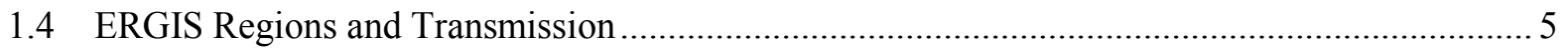

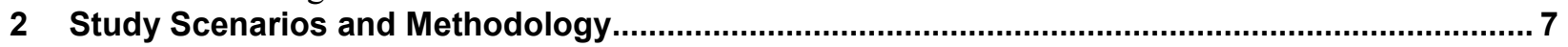

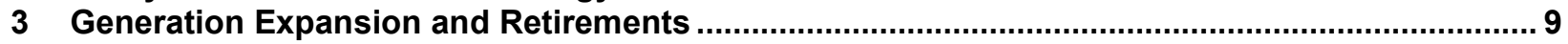

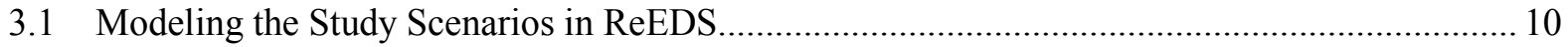

3.2 Thermal Fleet Retirement Assumptions and Results .......................................................... 11

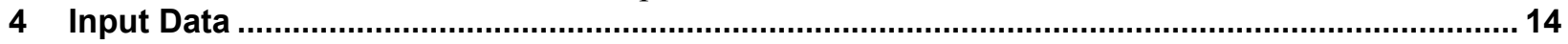

4.12010 Eastern Interconnection Model Development .............................................................. 14

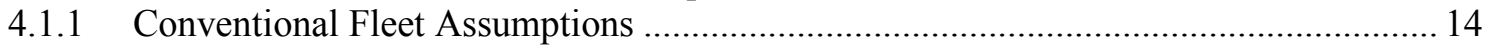

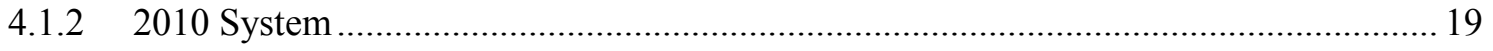

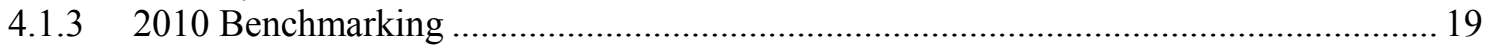

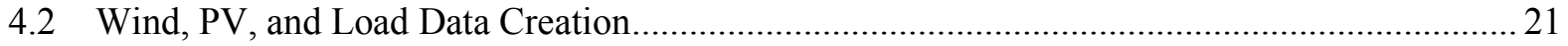

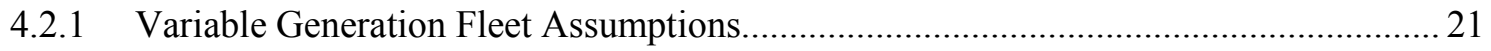

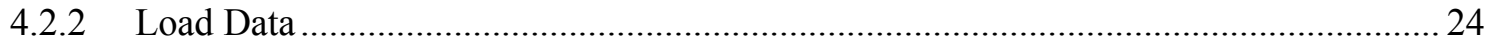

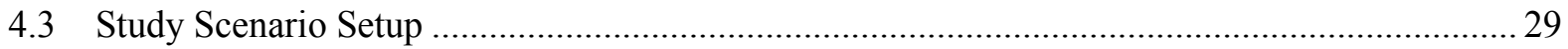

4.3.1 Conversion of ReEDS Thermal Fleet Results Into PLEXOS Generators ...................... 29

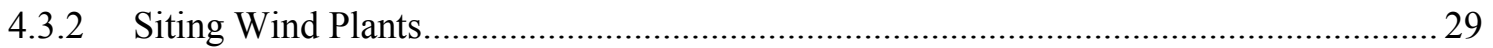

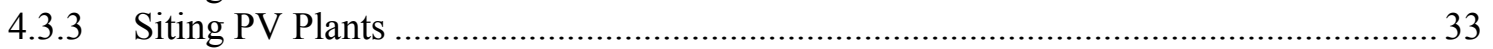

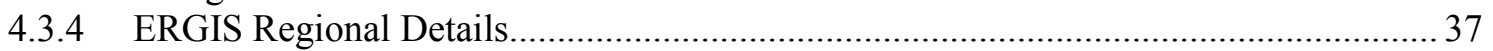

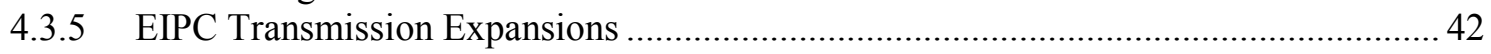

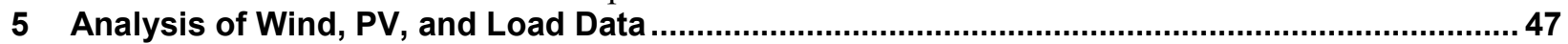

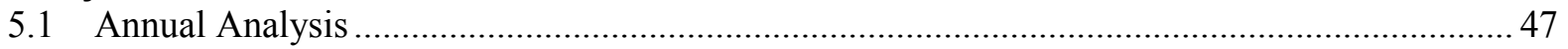

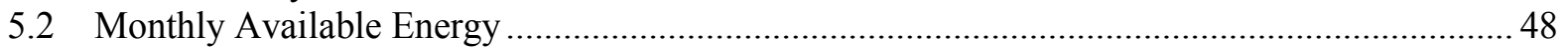

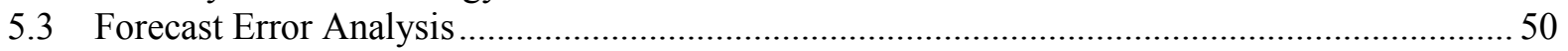

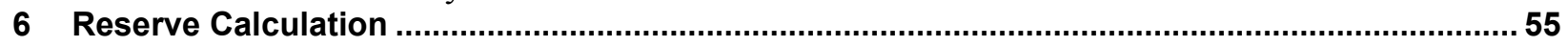

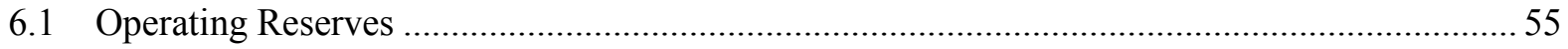

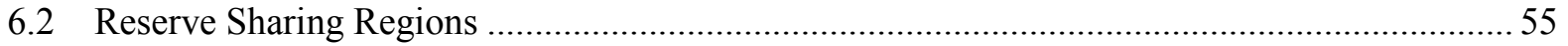

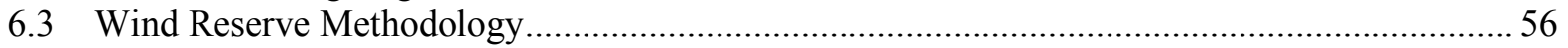

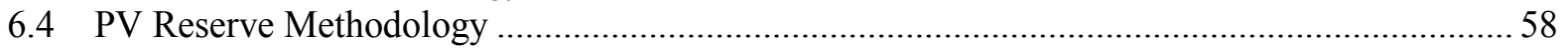

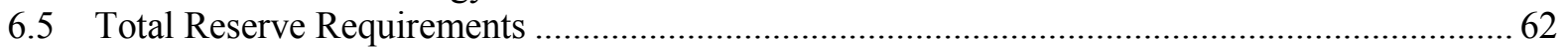

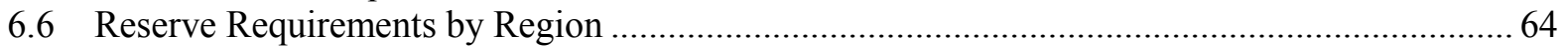

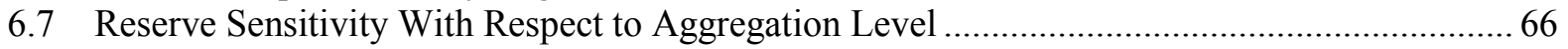

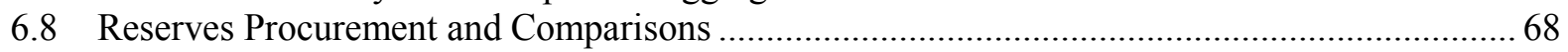

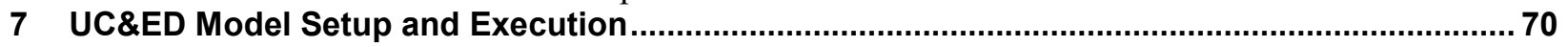

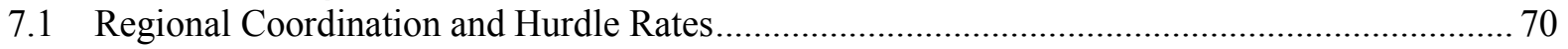

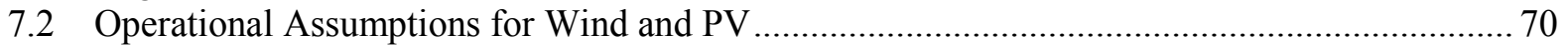

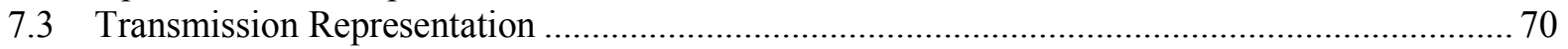

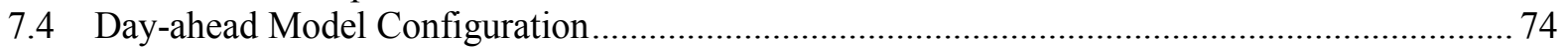

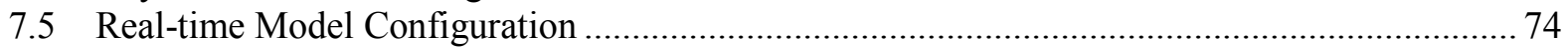

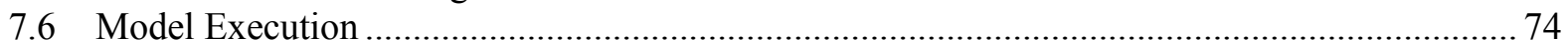

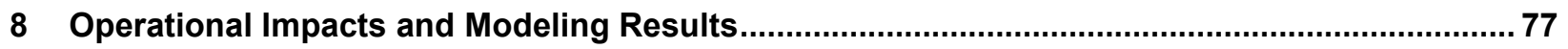

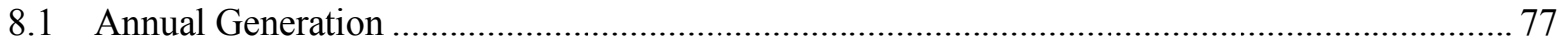

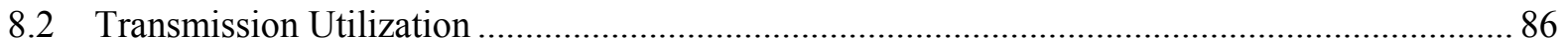

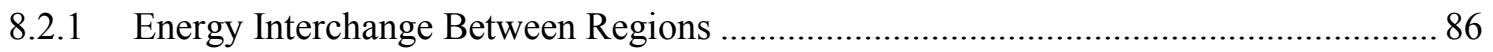

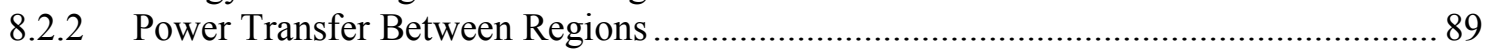

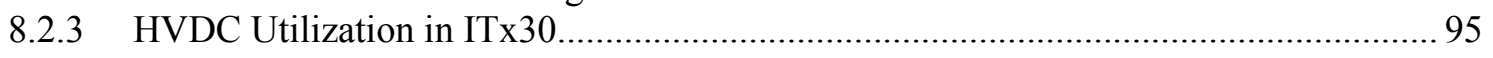

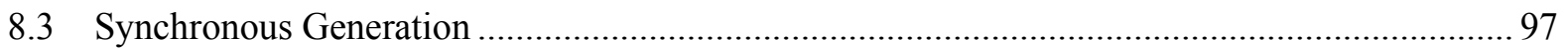




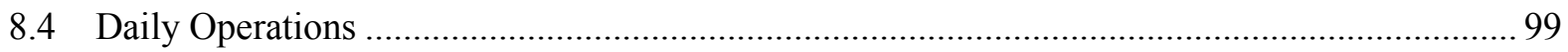

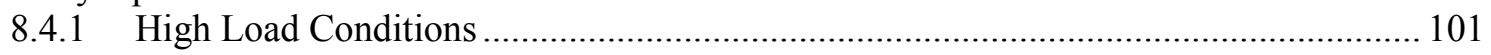

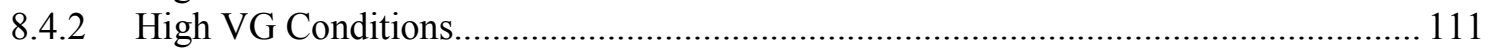

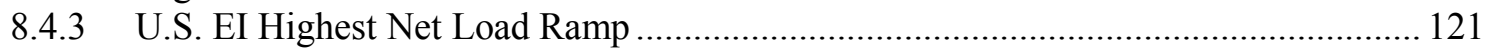

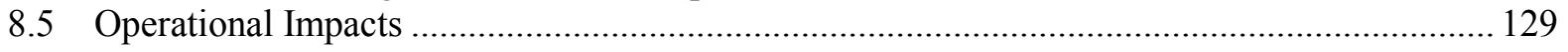

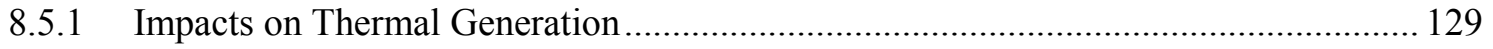

8.5.2 Generation from Hydro and Pumped Storage ........................................................... 135

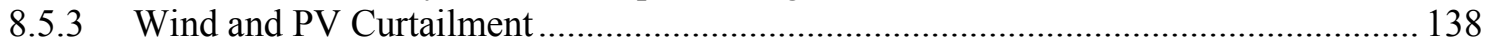

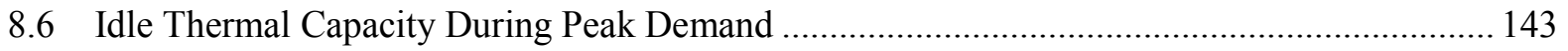

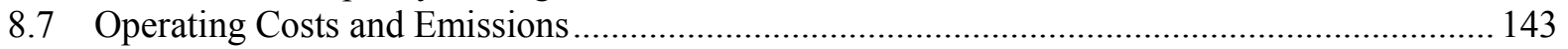

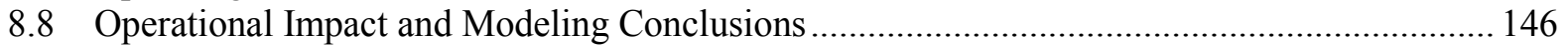

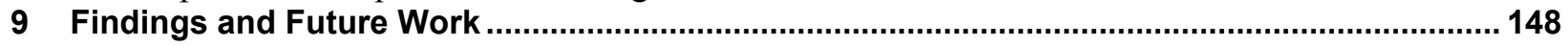

9.1 The operation of thermal and hydro generation changes as wind and PV increase. ................ 149

9.2 System operations at sunrise and sunset could follow different patterns................................. 150

9.3 Transmission flows will likely change more rapidly and more frequently with higher penetrations of wind and PV.

9.4 The operating practices of generators and transmission operators will be critical to realizing the total technical potential of the interconnection.

9.5 Advanced visualization tools are helpful for understanding spatially and temporally rich models. 156

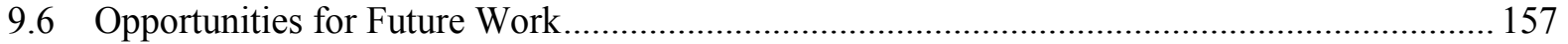

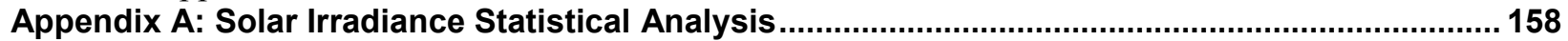

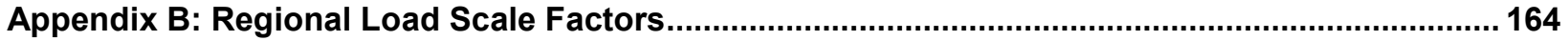

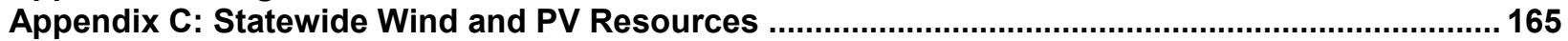

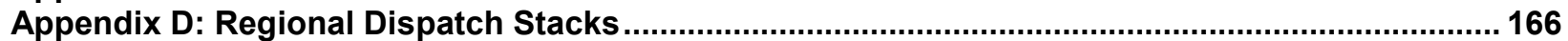

Appendix E: Regional real-time and day-ahead Commitment and Dispatch.................................190

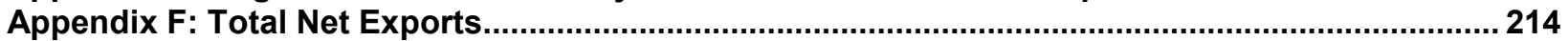

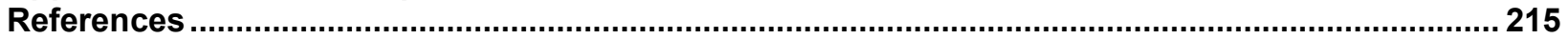




\section{List of Figures}

Figure 1. ERGIS Regions 5

Figure 2. Base transmission network of the Eastern Interconnection ................................................... 6

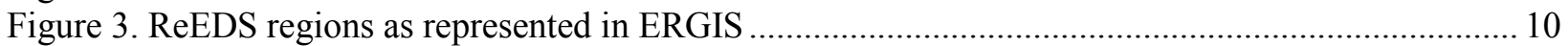

Figure 4. Distribution of full-load heat rates for coal-fired and gas-fired generators ............................... 15

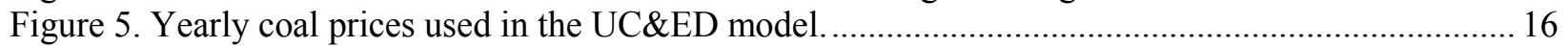

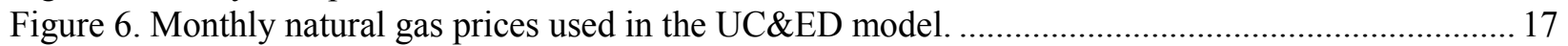

Figure 7. Example weekly hydro generation constraints for SEPA, SWPA, and USACE facilities .......... 18

Figure 8. Example monthly limits for Canadian hydro generators ...................................................... 19

Figure 9. Generation by fuel type and region in the benchmarking exercise compared to actual 2010 data

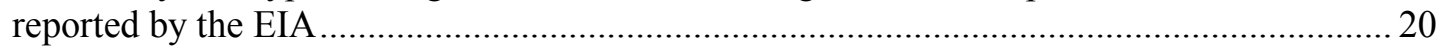

Figure 10. Map showing the net interchange patterns for the 2010 benchmarking exercise ..................... 21

Figure 11. Graph showing 5-minute wind generation data that was created from adding simulated noise to

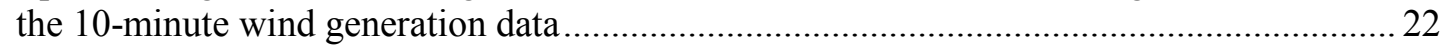

Figure 12. Filtering of high resolution data to get load variability ........................................................ 26

Figure 13. Comparison of various moving average window lengths on filter response for 8:30-10 a.m. for

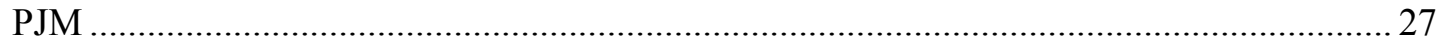

Figure 14. Variability results for high resolution datasets ......................................................... 28

Figure 15. Seasonal variability for Long Island, New York (LI), New York City, New York (NYC), ISO-

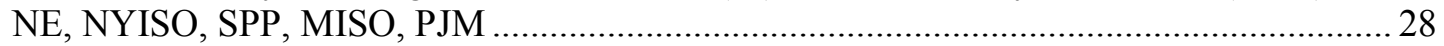

Figure 16. Installed wind capacity by state for each of the ERGIS scenarios: LowVG (first), RTx10

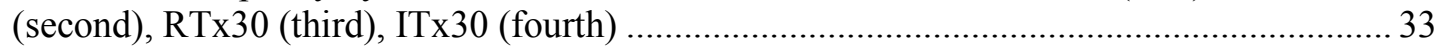

Figure 17. Maps of the installed PV capacity in the four ERGIS scenarios ............................................ 37

Figure 18. Map showing the EIPC Phase II Scenario 3 transmission expansion overlaid on the ERGIS regions. This expansion was used in the LowVG. ............................................................ 44

Figure 19. Map showing the EIPC Phase II Scenario 2 transmission expansion overlaid on the ERGIS regions. This expansion was used in RTx10 and RTx30 .................................................. 45

Figure 20. Map showing the EIPC Phase II Scenario 1 transmission expansion overlaid on the ERGIS

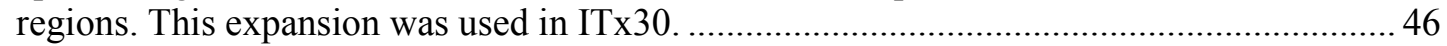

Figure 21. Duration plots for available energy from wind, PV, and total VG, and net load. Note that the yaxis scales are different on the each of these graphs.......................................................... 48

Figure 22. Monthly total VG available energy for the four scenarios. Note that the y-axis scales are

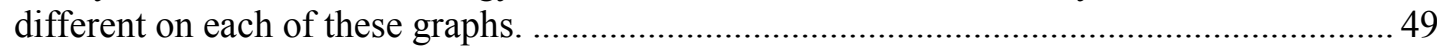

Figure 23. Wind, PV, and total VG available energy by month and scenario........................................ 49

Figure 24. Distribution of day-ahead wind forecast errors. Bins have widths of 2.5GW (left-hand plot) and 0.01 (right-hand plot)........................................................................................ 52

Figure 25. Distribution of day-ahead PV forecast errors. Bins have widths of 2.5GW (left-hand plot) and

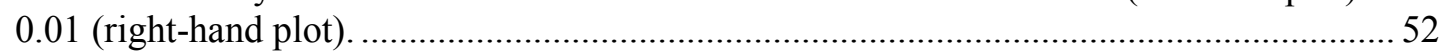

Figure 26. Distribution of total VG forecast errors. Bins have widths of 2.5GW (left-hand plot) and 0.01

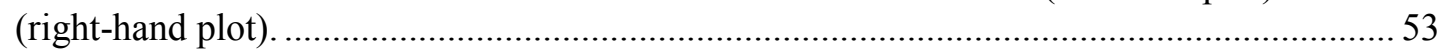

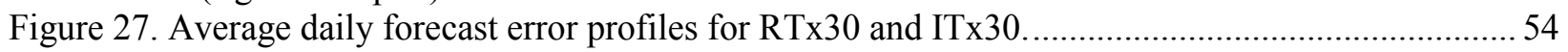

Figure 28. Regulation Reserve Sharing Regions .......................................................................... 56

Figure 29. Wind 10-minute forecast errors versus power output, with $95 \%$ confidence interval bands for

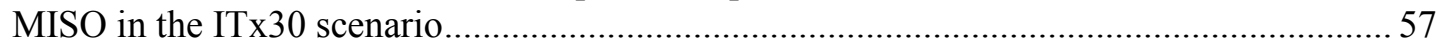

Figure 30. Wind power production and dynamic regulation reserve requirement for MISO in the ITx3058

Figure 31. Power and clear-sky power output and ramps for MISO in the ITx30 scenario ......................59

Figure 32. Graphical representation of short-term forecast for PV ................................................. 60

Figure 33. Ten-minute PV forecast error distribution for the persistence and the SPI-based methods for

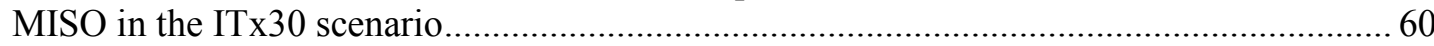


Figure 34. PV component of the regulation reserve requirements for the RTx30 and ITx30 scenarios for

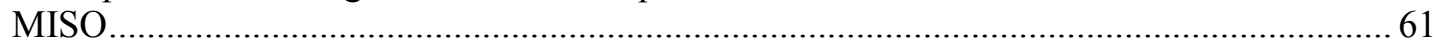

Figure 35. Dynamic PV regulation reserve requirements for MISO for the ITx30 scenario...................... 62

Figure 36. Power, regulation reserve for a few selected days in August, for MISO, by scenario .............. 64

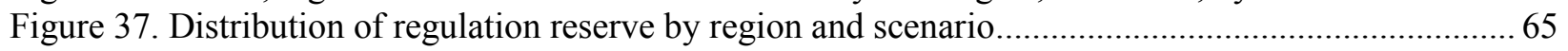

Figure 38. Regulation reserve duration curves by scenario and level of aggregation............................... 67

Figure 39. Map showing the ERGIS sub-regions, which were used as both transmission zones and load

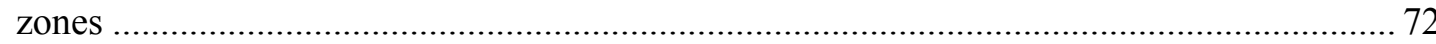

Figure 40. Map showing the transmission flowgates between the ERGIS sub-regions ........................... 73

Figure 41. Illustration of annual (blue) vs. decomposed (red) UC\&ED horizons ................................... 75

Figure 42. Computation times for annual simulations before and after partitioning and parallel

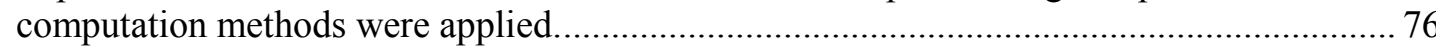

Figure 43. Annual generation by generator type for the U.S. Eastern Interconnection across all four

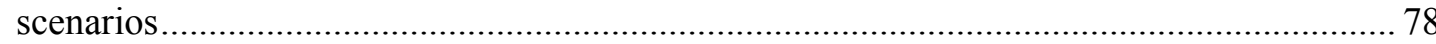

Figure 44. Differences between LowVG and other scenarios in annual generation by generator type for

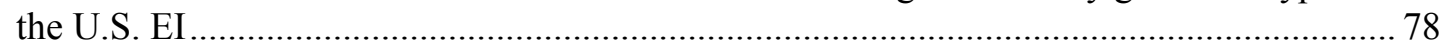

Figure 45. Instantaneous penetration of $\mathrm{VG}$ as a percent of total generation ........................................... 80

Figure 46. Duration curve of VG penetration for all scenarios for the U.S. EI ..................................... 81

Figure 47. Annual load and generation by generator type for all U.S. EI regions................................... 82

Figure 48. Difference in annual generation between LowVG and other scenarios, by generator type (note

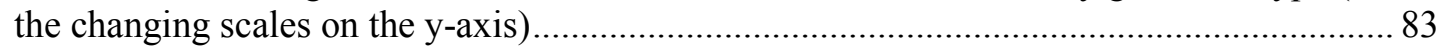

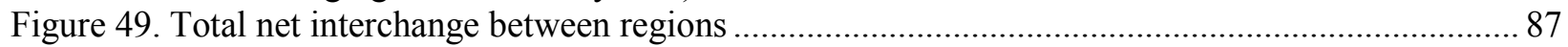

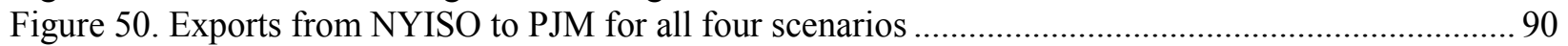

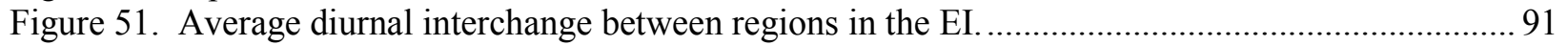

Figure 52. Annual duration curves of exports for select interfaces ...................................................... 93

Figure 53. Annual duration curves of exports for the FRCC-SERC interface....................................... 94

Figure 54. The ITx30 transmission expansion includes six HVDC lines connecting the MISO and SPP

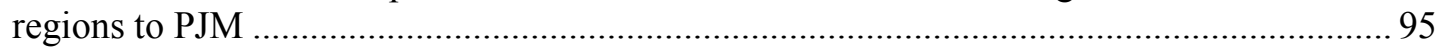

Figure 55. Energy transferred across select HVDC interties in ITx30 scenario ..................................... 96

Figure 56. Instantaneous VG penetration as a percent of online capacity ........................................... 98

Figure 57. Key for interpreting day-ahead and real-time dispatch and commitment figures ................. 100

Figure 59. System dispatch in each scenario for the EI during high load conditions............................ 104

Figure 60. Combined commitment and dispatch of the EI for PV, wind, CT/Gas boiler, CC, hydro, and

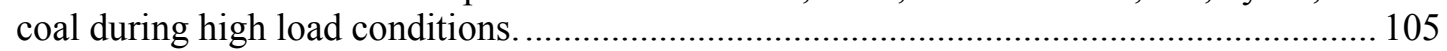

Figure 61. Combined commitment and dispatch of SPP for PV, wind, CT/Gas boiler, CC, hydro, and coal

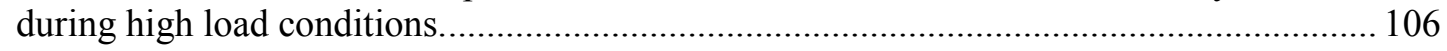

Figure 62. Combined commitment and dispatch of MISO for PV, wind, CT/Gas boiler, CC, hydro, and

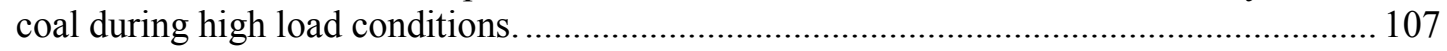

Figure 63. Combined commitment and dispatch of PJM for PV, wind, CT/Gas boiler, CC, hydro, and coal

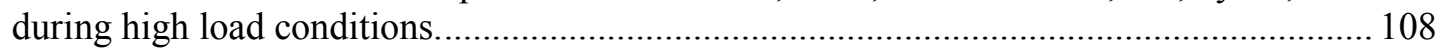

Figure 64. Combined commitment and dispatch of ISO-NE for PV, wind, CT/Gas boiler, CC, hydro, and coal during high load conditions. .................................................................................... 109

Figure 65. Combined commitment and dispatch of NYISO for PV, wind, CT/Gas boiler, CC, hydro, and

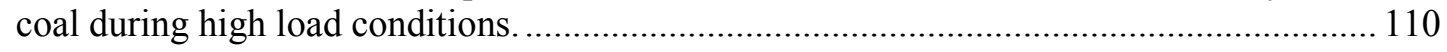

Figure 66. System dispatch in each scenario for FRCC during high load conditions.............................. 111

Figure 67. System dispatch in each scenario for the EI during high VG conditions. .............................. 114

Figure 68. Combined commitment and dispatch of the EI for PV, wind, CT/Gas boiler, CC, hydro, and

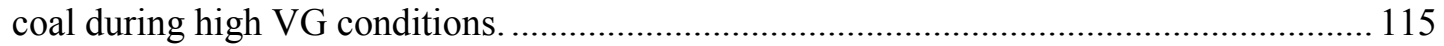

Figure 69. System dispatch in each scenario for MISO during high VG conditions.............................. 116

Figure 70. System dispatch in each scenario for PJM during high VG conditions................................. 117

Figure 71. System dispatch in each scenario for SERC during high VG conditions............................... 118 
Figure 72. System dispatch in each scenario for FRCC during high VG conditions............................. 119

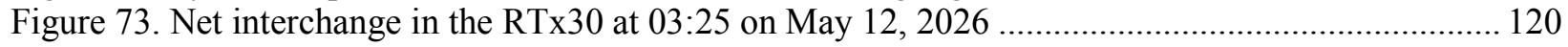

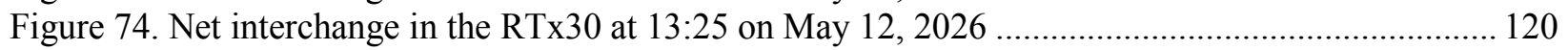

Figure 75. System dispatch in each scenario for the EI during high net load ramp conditions ............... 123

Figure 76. Combined commitment and dispatch of the EI for PV, wind, CT/Gas boiler, CC, hydro, and coal during high net load ramp conditions ...................................................................... 124

Figure 77. Combined commitment and dispatch of the MISO for PV, wind, CT/Gas boiler, CC, hydro, and coal during high net load ramp conditions ............................................................... 125

Figure 78. Combined commitment and dispatch of the PJM for PV, wind, CT/Gas boiler, CC, hydro, and coal during high net load ramp conditions ........................................................................ 126

Figure 79. Combined commitment and dispatch of the SPP for PV, wind, CT/Gas boiler, CC, hydro, and coal during high net load ramp conditions ..................................................................... 127

Figure 80. System dispatch and net interchange for the EI in all 4 scenarios at 12:00 November 24, 2026128

Figure 81. System dispatch and net interchange for the EI in all 4 scenarios at 14:25 on November 24,

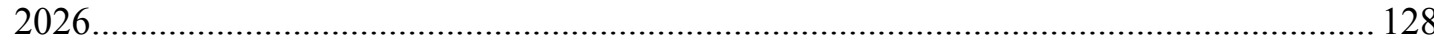

Figure 82. Capacity factors of thermal units for the total fleet in the U.S. EI (top), Average output of committed units (middle) and percent of online time at minimum generation (bottom) ..... 131

Figure 83. Number of ramps for the total U.S. EI and U.S. regional fleets divided by the energy produced 133

Figure 84. Capacity-starts (capacity in GW multiplied by number of starts, for each unit) for the U.S. EI generators by fuel type (top), and the average number of days online per start (bottom).... 134

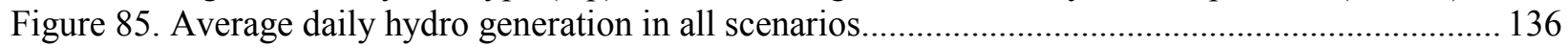

Figure 86. Average daily pumped hydro operation in all scenarios................................................... 137

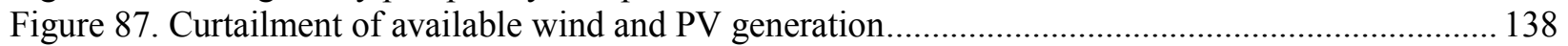

Figure 88. U.S. EI duration curve for wind and PV curtailment ..................................................... 139

Figure 89. Monthly VG curtailment profiles for each scenario ....................................................... 139

Figure 90. Annual average daily profiles of VG curtailment for the whole U.S. EI and for each U.S.

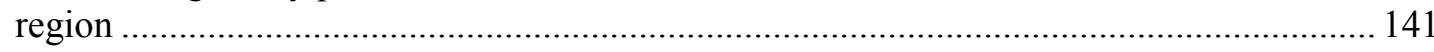

Figure 91. Seasonal average daily profiles of VG curtailment for the whole U.S. EI and for each U.S. EI

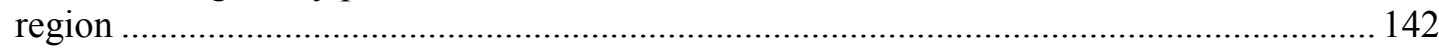

Figure 92. Annual operating costs for each of the scenarios ........................................................ 144

Figure 93. Carbon dioxide emissions for the U.S. EI in each scenario (left), and change in carbon dioxide

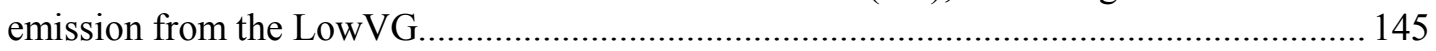

Figure 94. Capacity factors of thermal units for the total fleet in the U.S. EI (top), Average output of committed units (middle) and percent of online time at minimum generation (bottom) ..... 149

Figure 95. Dispatch for the U.S. EI during high wind and PV conditions.......................................... 151

Figure 96. Net interchange in the LowVG on May 12, 2026 at 13:25 .............................................. 153

Figure 97. Net interchange in the RTx30 on May 12, 2026 at 13:25 ................................................ 153

Figure 98. Day-ahead and real-time results for a period of high wind and PV generation...................... 155

Figure 99. Sample screen from "kaleidoscope" visualization tool, one of two tools developed for ERGIS156 


\section{List of Tables}

Table 1. Select Assumptions in Previous UC\&ED Studies of the Eastern Interconnection ......................... 3

Table 2. ERGIS Includes Four Scenarios with Different Levels of Wind, PV, and Transmission Capacity

Expansion. Values are the percent of annual generation. .................................................... 7

Table 3. Maximum Economic Lifetime of Thermal Plants in ReEDS ...................................................... 12

Table 4. U.S. EI Thermal Capacity Retired by ReEDS LowVG by 2026 ............................................ 12

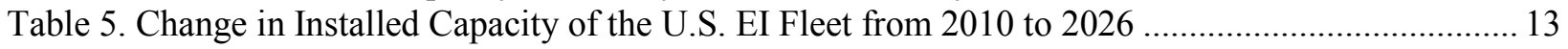

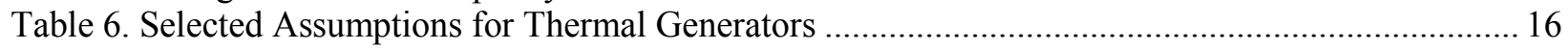

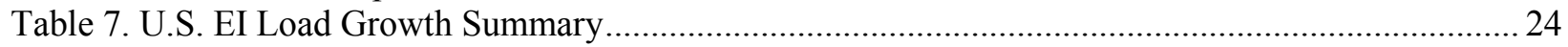

Table 8. Actual Load Datasets Used to Characterize Sub-hourly Load Variation .................................. 25

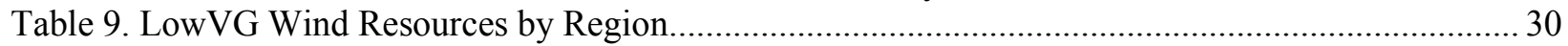

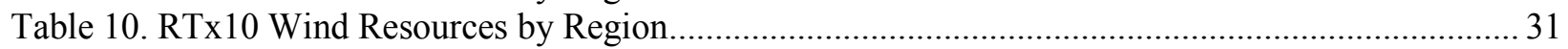

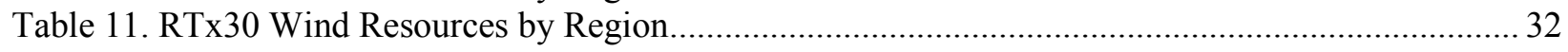

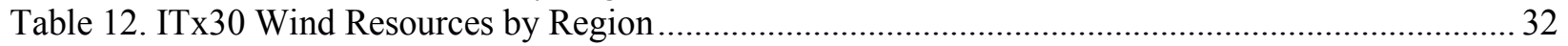

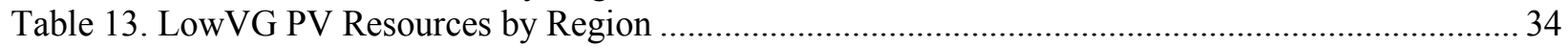

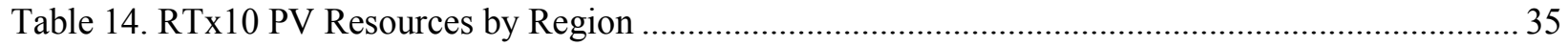

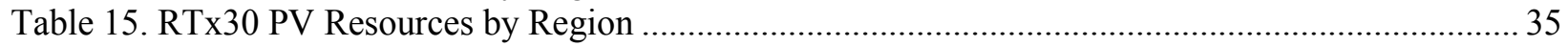

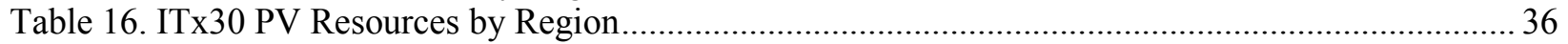

Table 17. Installed Capacity of the EI in 2010 and Each ERGIS Scenario .............................................. 38

Table 18. Installed Capacity of the Canadian Provinces in 2010 and Each ERGIS Scenario .................... 38

Table 19. Installed Capacity of FRCC in 2010 and Each ERGIS Scenario................................................ 39

Table 20. Installed Capacity of SERC in 2010 and Each ERGIS Scenario ................................................ 39

Table 21. Installed Capacity of MISO in 2010 and Each ERGIS Scenario ............................................... 40

Table 22. Installed Capacity of SPP in 2010 and Each ERGIS Scenario .................................................. 41

Table 23. Installed Capacity of PJM in 2010 and Each ERGIS Scenario................................................. 41

Table 24. Installed Capacity of NYISO in 2010 and Each ERGIS Scenario........................................... 42

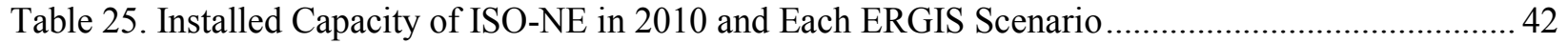

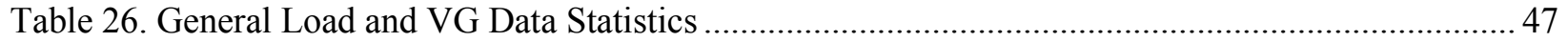

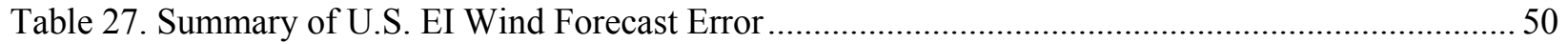

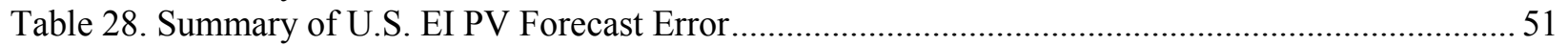

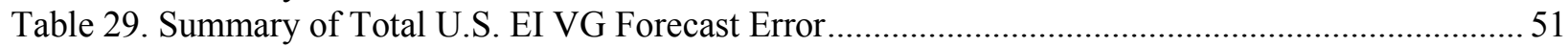

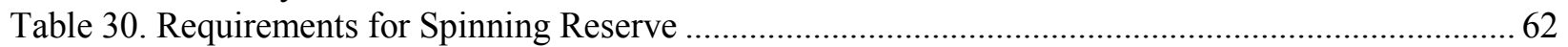

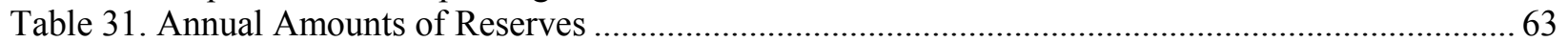

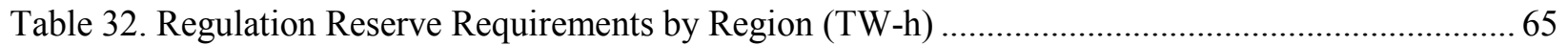

Table 33. Level of Aggregation Studied for Southern Regions............................................................ 66

Table 34. Total Reserve Requirements for the Five Selected Regions at Different Aggregation Levels

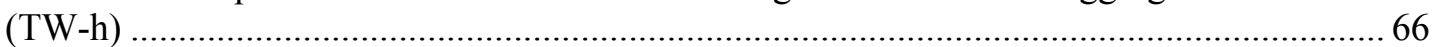

Table 35. Peak Load and Capacity of Regulating Reserves Held in each ERGIS Scenario as Compared to

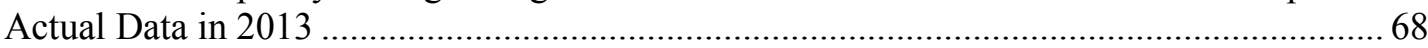

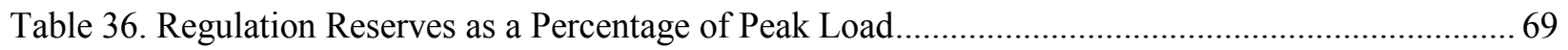

Table 37. Fuel Usage by Coal and Gas Generators in ERGIS Scenarios (quadrillion BTUs)................... 81

Table 38. Difference in Coal- and Gas-fueled Generation from LowVG for FRCC (TWh) ..................... 85

Table 39. Difference in Coal- and Gas-fueled Generation from LowVG for ISO-NE (TWh) .................. 85

Table 40. Difference in Coal- and Gas-fueled Generation from LowVG for MISO (TWh) ..................... 85

Table 41. Difference in Coal- and Gas-fueled Generation from LowVG for NYISO (TWh) ................... 85

Table 42. Difference in Coal- and Gas-fueled Generation from LowVG for PJM (TWh)....................... 86

Table 43. Difference in Coal- and Gas-fueled Generation from LowVG for SERC ................................ 86

Table 44. Difference in Coal- and Gas-fueled Generation from LowVG for SPP .................................. 86

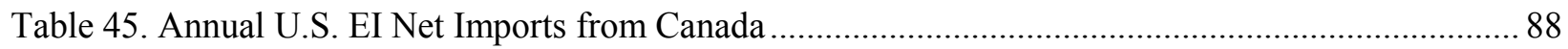

Table 46. Annual Imports and Exports for Each Region .................................................................. 88

xii 
Table 47. Daily Number of Changes in the Direction of Net Flow between Pairs of Regions.................. 89

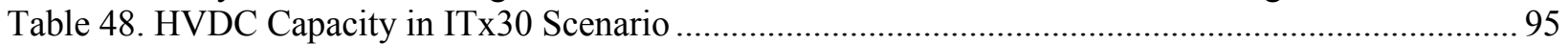

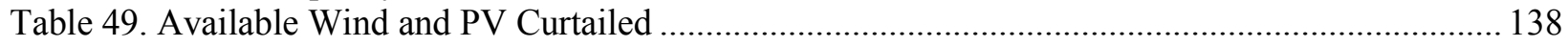

Table 50. Idle Thermal Capacity During Peak Demand in Each Scenario ........................................... 143

Table 51. Comparison of Operating Costs and Emissions with Increasing Wind and PV Generation..... 146

Table 52. Fit Parameters for the Regions Mapped in Figure 10 ......................................................... 161

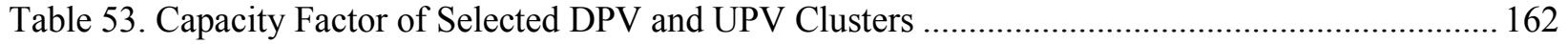

Table 54. Final Load Scale Factors for Each of the ERGIS Sub-Regions............................................. 164

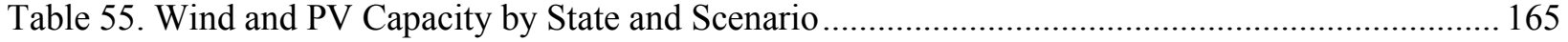

Table 56. Total net exports (TWh) between ERGIS macro-regions..................................................... 214 


\section{Introduction}

The Eastern Interconnection (EI) is one of the largest power systems in the world, and its size and complexity have historically made it difficult to study in high levels of detail in a modeling environment. In order to understand how this system might be impacted by high penetrations (30\% of total annual generation) of wind and solar photovoltaic (PV) ${ }^{1}$ during steady state operations, the National Renewable Energy Laboratory (NREL) and the U.S. Department of Energy (DOE) conducted the Eastern Renewable Generation Integration Study (ERGIS). This study investigates certain aspects of the reliability and economic efficiency problem faced by power system operators and planners. Specifically, the study models the ability to meet electricity demand at a 5-minute time interval by scheduling resources for known ramping events, while maintaining adequate reserves to meet random variation in supply and demand, and contingency events. To measure the ability to meet these requirements, a unit commitment and economic dispatch (UC\&ED) model is employed to simulate power system operations. The economic costs of managing this system are presented using production costs, a traditional UC\&ED metric that does not include any consideration of long-term fixed costs.

ERGIS simulated one year of power system operations to understand regional and sub-hourly impacts of wind and PV by developing a comprehensive UC\&ED model of the EI. In the analysis, it is shown that, under the study assumptions, generation from approximately $400 \mathrm{GW}$ of combined wind and PV capacity can be balanced on the transmission system at a 5-minute level. In order to address the significant computational burdens associated with a model of this detail we apply novel computing techniques to dramatically reduce simulation solve time while simultaneously increasing the resolution and fidelity of the analysis. Our results also indicate that high penetrations of wind and PV (collectively variable generation (VG)), significantly impact the operation of traditional generating resources and cause these resources to be used less frequently and operate across a broader output range because wind and PV have lower operating costs and variable output levels.

\subsection{Background and Objectives}

Wind and PV generation are the fastest growing electricity resources in the United States and in the U.S. EI. ${ }^{2}$ The U.S. Energy Information Administration (EIA) estimates that renewable electric sources accounted for more than $61 \%$ of all new U.S. electricity capacity installations in 2013, up from 57\% in 2008 and 4\% in 2004 (EIA 2015). The adoption of these technologies has been spurred by state renewable portfolio standards (RPS), federal policies affecting the tax structures of wind and PV projects, renewable technology advancements, and cost decreases.

ERGIS is an operational impacts study; it is not designed to identify the most optimal mix of generation and transmission, or analyze dynamic power system characteristics at a sub-5-minute timescale. A scenario-based approach is taken to understand system-wide operational impacts of high amounts of VG on the EI under different transmission grid configurations. Typical operational impacts investigated in this study include the impact on resource generation, VG

\footnotetext{
${ }^{1}$ Concentrating solar power (CSP) was not considered in this study.

${ }^{2}$ The U.S. EI refers only to the U.S. portion of the EI. Many parts of our analysis focus on the impacts to this area because expansion of wind and PV was limited to the U.S. in ERGIS, although the whole EI (including parts of Canada) is represented in our UC\&ED model.
} 
curtailment, ramping, cycling. ERGIS adopted four capacity expansion scenarios (developed in cooperation with the technical review committee (TRC)) and three transmission expansions (developed independently by the Eastern Interconnection Planning Collaborative (EIPC)) and then analyzed operation of the EI in those scenarios. The scenarios studied in this project are designed to allow comprehensive analysis of potential integration issues, and are not a forecast of wind and PV deployment based on the cost of those resources. ERGIS reflects considerable effort to model many existing operational constraints with respect to reserve products, interregional cooperation, and thermal and hydro plant capabilities.

We used several advanced modeling and computational techniques in ERGIS, to improve upon simplifying assumptions about power system operations that have been used in previous comparable analyses. A few of the common approximations used in UC\&ED models include zonal transmission, hourly simulation, simplified optimization heuristics, and generator aggregation. While many of these assumptions have been accepted based on historical necessity, there is growing concern that these simplifications may underestimate the impact of high penetrations of wind and PV generation. We advanced the state-of-the art by including a nodal transmission representation, simulation of 5-minute operations for an entire year, use of mixedinteger programming (MIP) and co-optimization of energy and ancillary services. In order to overcome computational constraints associated with increasing the temporal and spatial resolution of the study, NREL applied novel techniques developed by Barrows et al. (2014) to parallelize the simulations and dramatically reduce computational limitations.

NREL used two models for this study. The primary model was PLEXOS, a UC\&ED production cost simulation model (PLEXOS 2014). PLEXOS optimizes operation of the generators to minimize overall production cost while observing various constraints such as generator, reserve requirements, and transmission limits. PLEXOS was used to simulate an entire year of power system operations at a 5-minute temporal resolution and down to individual transmission buses. The second model was NREL's Regional Energy Deployment System (ReEDS) (Short et al. 2011). ReEDS is a capacity expansion model that optimizes expansion of all types of traditional generators while accurately representing the spatial and temporal availability of renewable generation technologies. ReEDS was used to determine the quantity and location of all generation additions and retirements in the study. The coordinated use of these models results in a detailed simulation of power system operations. To perform the power system modeling, NREL utilized wind and PV datasets created with meso-scale weather models (see Section 4.2).

The scenarios simulated in ReEDS or PLEXOS are not intended to be forecasts or predictions; rather, ReEDS and PLEXOS provide self-consistent frameworks to assess the potential impacts of different technology, market, and policy conditions on technology deployment and system operation.

\subsection{Improvements and Limitations}

The ERGIS study joins a growing list of VG integration studies that have examined part or all of the EI. One of the goals of ERGIS was to add enhanced simulation methods to increase confidence in the ability of integrating increased amounts of VG. To provide a brief overview of modeling enhancements, Table 1 lists several assumptions used in this study, as well as five previous studies: the Eastern Wind Integration and Transmission Study (EWITS), The Manitoba Hydro Wind Synergy Study (Bakke, Zhou, and Mudgal, n.d.), The Eastern Interconnection 
Planning Collaborative Phase 2 Report (EIPC 2012), the Minnesota Renewable Integration and Transmission Study (MRITS)(GE 2014b), and PJM Renewable Generation Integration Study (PRIS) (GE 2014a) projects. We reviewed published reports for each project and worked with TRC members and authors to verify the listed information. In aggregate, the improvements in the ERGIS study represent an increase in temporal, geographic, and technical fidelity. First, ERGIS expands the range of resources analyzed by simulating large-scale adoption of PV in addition to wind in the U.S. EI. This increases the number of generators on the system considerably, from previous studies, and thus the complexity of the UC\&ED. Next, the study narrows the temporal resolution to 5 minutes in order to understand the sub-hourly impact of these resources on system operations. This time resolution reflects the dispatch interval of existing regional transmission organizations and independent system operators (RTOs/ISOs). ERGIS also increases the spatial resolution of the model to include all synchronous components of the Eastern Interconnection and Québec Interconnection (collectively, EI), on a comparable basis. This increases the number of transmission facilities and generators in the model significantly. Previous work by Dean, Drayton, and Gallachóir (2014) indicate that sub-hourly modeling is an important factor in accurately characterizing the impact of wind on production costs and thermal plant operations. Further work by Stoll et al. (2016) analyzed the effects of optimization technique, temporal resolution, and intra-day commitment on several key metrics for integration studies. They found that operational metrics such as starts, time online, and ramping were significantly impacted by the use of continuous linear optimization and hourly temporal resolutions.

Table 1. Select Assumptions in Previous UC\&ED Studies of the Eastern Interconnection

\begin{tabular}{|c|c|c|c|c|c|c|}
\hline Study & 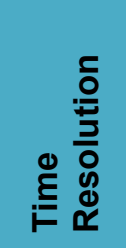 & 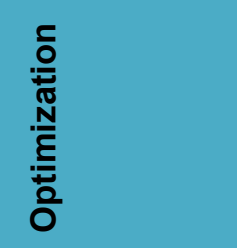 & 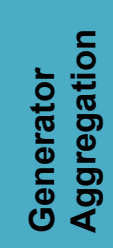 & 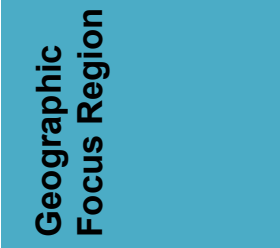 & क⿺ & 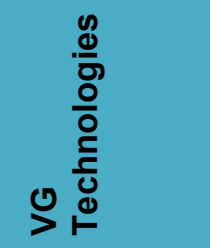 \\
\hline $\begin{array}{l}\text { EIPC } \\
\text { Phase } 2\end{array}$ & Hourly & $\begin{array}{l}\text { MIP } \\
\text { approximation }\end{array}$ & Yes & $\mathrm{El}$ & GE MAPS & Wind and $P V$ \\
\hline ERGIS & $\begin{array}{l}\text { Sub- } \\
\text { hourly }\end{array}$ & MIP & No & $\mathrm{El}$ & PLEXOS & Wind and $P V$ \\
\hline EWITS & Hourly & $\begin{array}{l}\text { MIP } \\
\text { approximation }\end{array}$ & No & $\begin{array}{l}\text { US EI, excluding } \\
\text { Florida }\end{array}$ & PROMOD & Wind \\
\hline $\begin{array}{l}\text { Manitoba } \\
\text { Synergy }\end{array}$ & $\begin{array}{l}\text { Sub- } \\
\text { hourly }\end{array}$ & $\begin{array}{l}\text { MIP } \\
\text { approximation }\end{array}$ & No & $\begin{array}{l}\text { El excluding } \\
\text { eastern Canada, } \\
\text { ISO-NE, and } \\
\text { Florida }\end{array}$ & PLEXOS & Wind \\
\hline MRITS & Hourly & $\begin{array}{l}\text { MIP } \\
\text { approximation }\end{array}$ & No & $\begin{array}{l}\text { El excluding } \\
\text { eastern Canada, } \\
\text { ISO-NE, and } \\
\text { Florida }\end{array}$ & PLEXOS & Wind and $P V$ \\
\hline PRIS & Hybrid & $\begin{array}{l}\text { MIP } \\
\text { approximation }\end{array}$ & No & EI-PJM focus & GE MAPS & Wind and $\mathrm{PV}$ \\
\hline
\end{tabular}


These improvements represent a substantial increase in computation burden, which required adoptions of new techniques described in Barrows et al. (2015) and also Section 7.6. Despite substantial improvements in the modeling framework and methods, which are explained in more detail later in the report, there are still several assumptions that create limitations to our modeling results. In particular, ERGIS assumes generating resources are co-optimized for energy and ancillary services (regulation and spinning/contingency reserves) across the whole EI according to assumed variable costs and forecasts for fuel prices. In reality, each region is optimizing individually with trades between regions, causing seams between the regions. Similarly, ERGIS only analyzes one meteorological year (2006) of operations and may not fully capture the range of possible extreme weather events. Regulation and spinning reserves are used in the study, but only to assure sufficient reserve capacity is available for operations; we do not simulate the activation of any reserves for contingencies or sub-5-minute variability. Further, the study does not constitute any analysis of N-1 contingencies nor does it evaluate operation in the sub-5minute and millisecond timescales; e.g. automated generator control, frequency response, or transient stability. The study conservatively assumed that hydroelectric generation is not used to provide reserves, as current constraints and practices for dam water use vary by region, with some regions using hydropower for reserves and others not.

The study also lacks consideration of long-term strategic economic behavior and relies on generalized unit-specific data. Specifically, ERGIS does not include detailed information about bilateral contracts, self-scheduling, and different operational practices in different regions. ERGIS ignores voltage and stability constraints included in an alternating current (AC) powerflow in favor of the simplified DC power flow model. The load assumptions used in the study had two notable limitations. First, load forecast data were not available so perfect hourly load forecasts were assumed. This means that load in the day ahead is exactly the same as the hourly real time load. However, the 5-minute real-time load varies based on the methods described in Section 4.2.2. Second, the study did not include any demand response. This is a conservative assumption because demand response is becoming increasingly common resource in the EI. Furthermore, the evaluation and implementation of future demand response resources was beyond the scope of this study. As an operational impact study, ERGIS does not include associated capital costs in its analysis. Instead of analyzing capital costs, we assumes certain generation and transmission futures and focuses the analysis on the ability of the system to operate with the assumed levels of wind and PV. Similarly, we do not evaluate the financial viability of generation entities as a result of studied scenarios. Additional uncertainties include the amount and location of wind and PV generation, transmission system additions, thermal generation retirements and additions, and natural gas and coal prices, all of which were used and modeled by ReEDS but are subject to uncertainty. Fuel for gas and coal generation was assumed to be sufficient to meet demand. Constraints on natural gas transportation may limit fuel availability. In addition, this analysis assumed wind and PV technology that was being widely deployed at the time this analysis began, and does not include recent and forecasted technology improvements, e.g. larger wind turbines.

\subsection{Technical Review}

NREL convened a TRC to assist in the development of assumptions and validation of methodology and findings. Consisting of representatives from every region in the interconnection, as well as other industry experts, the TRC met on a quarterly basis, for three 
years, to review the development of the wind and PV datasets, regional operational practices, thermal plant characteristics, setup and configuration of models, and transmission representation. Working groups met on four occasions to fine-tune assumptions regarding the Canadian system, thermal plant characteristics, hydropower plants, and transmission. A key role of the TRC on this project was to help ensure that assumptions and methods were accurately implemented in the model. The TRC played a critical role in guiding the study, analyzing study results, and reviewing this final report.

\subsection{ERGIS Regions and Transmission}

Figure 1 shows the geographic scope of ERGIS. The EI extends from Nova Scotia in the northeast and Florida in the south to eastern portions of New Mexico and Montana. The Québec Interconnection, which is asynchronous to the U.S. EI, is also included in the study because interchange with the Québec Interconnection heavily influences operations in the northeast United States. The footprint for each of the study regions was developed based on 2013 RTO/ISO regions and NERC regions for non-RTO/ISO regions. The base transmission configuration for the study area includes all transmission in-service in 2010, which is shown in Figure 2.

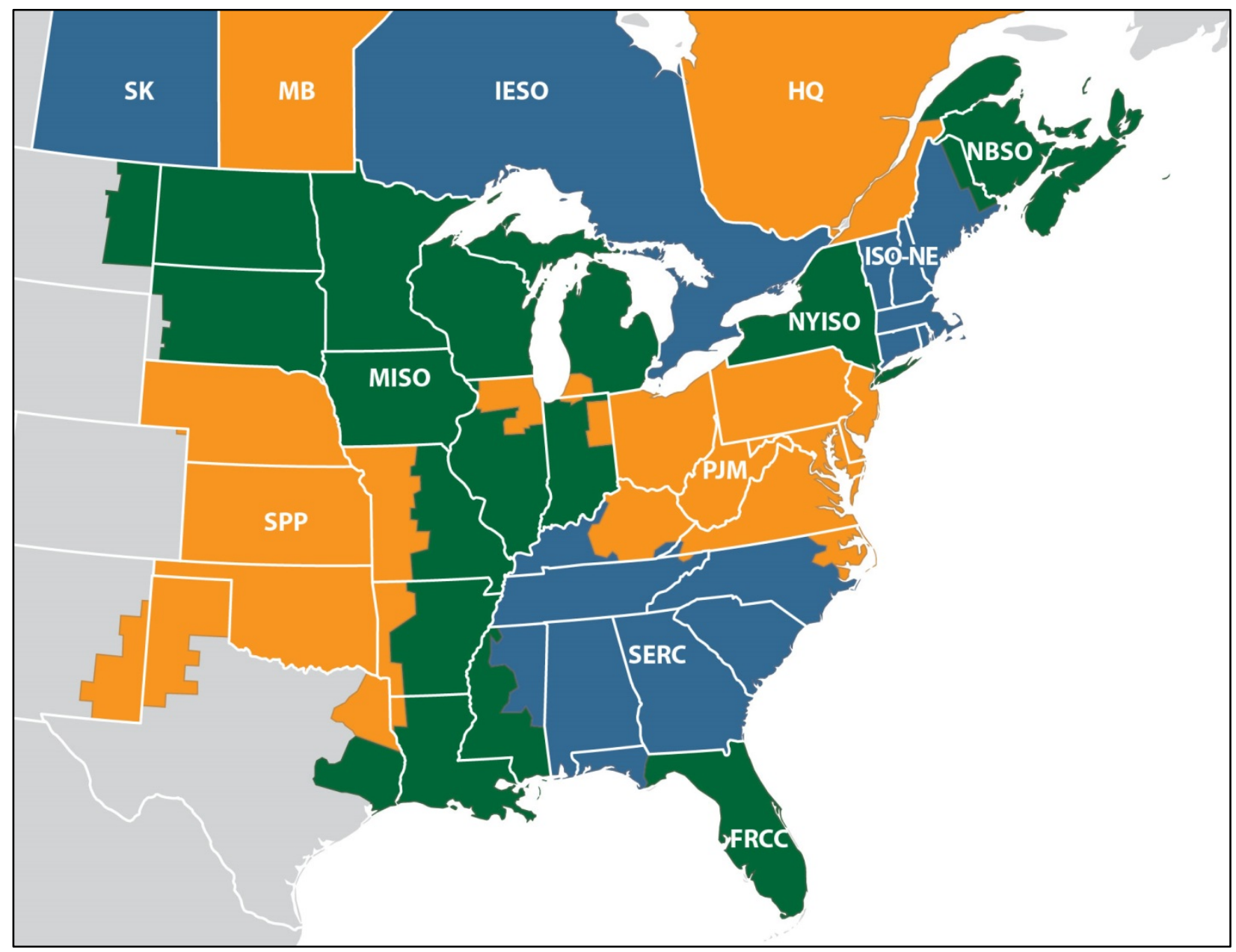

Figure 1. ERGIS Regions 


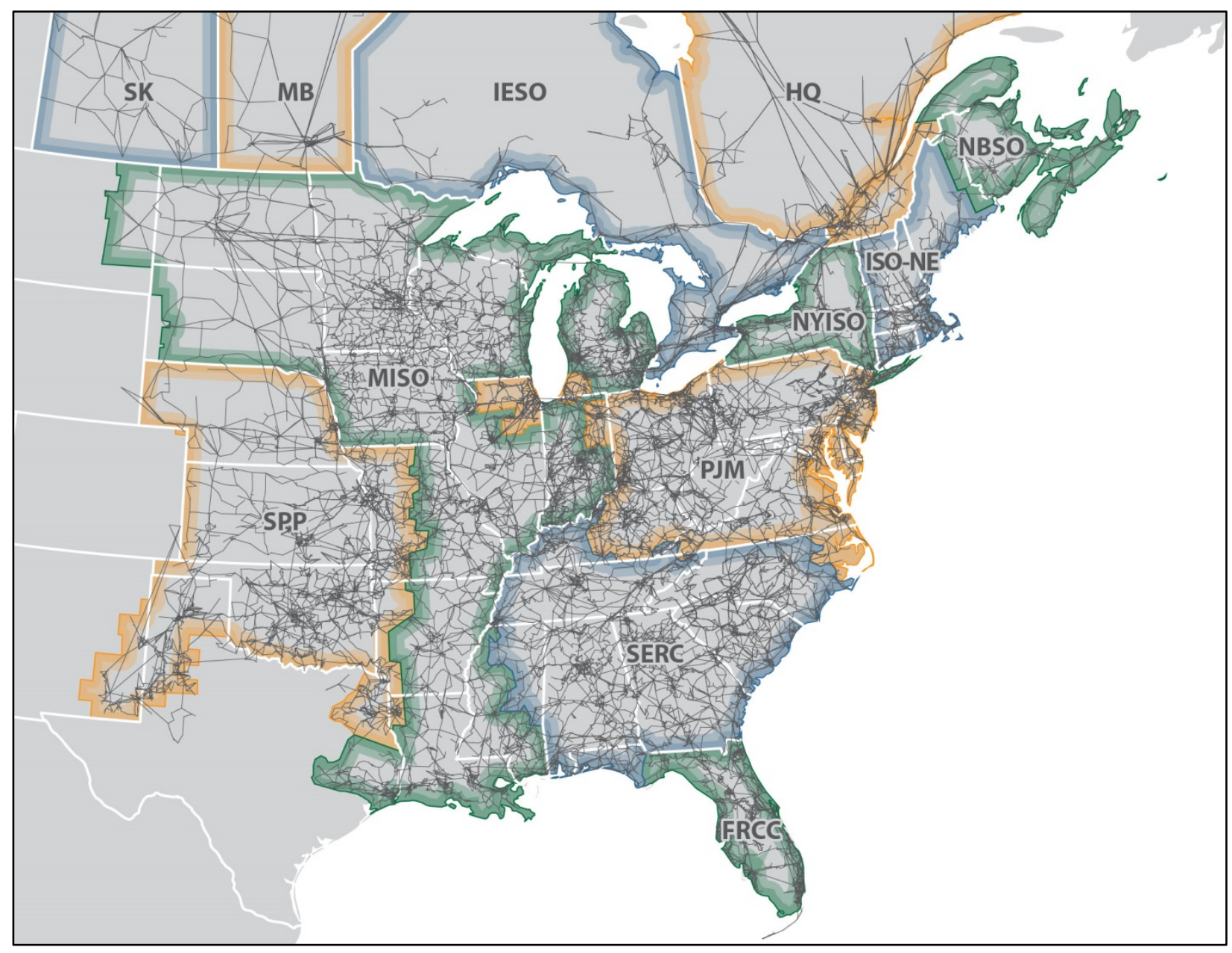

Figure 2. Base transmission network of the Eastern Interconnection 


\section{Study Scenarios and Methodology}

We developed the four study scenarios through a consensus process with the TRC. With their assistance, we identified a variety of qualitative characteristics to include in framing potential power system conditions in the next 10-15 years. All scenarios assume two key elements: (1) modest load growth and (2) significant retirements of coal and natural gas generation. These scenarios were developed to create bookend cases for studying $30 \%$ wind and PV that could reflect how inter-regional and regional policy decisions could drive wind and PV development. The qualitative characteristics of the four generation and transmission scenarios are outlined in Table 2.

Table 2. ERGIS Includes Four Scenarios with Different Levels of Wind, PV, and Transmission Capacity Expansion. Values are the percent of annual generation.

\begin{tabular}{|c|c|c|c|c|}
\hline Scenario & Wind & PV & Total $^{3}$ & Attributes \\
\hline LowVG & $3 \%$ & $0 \%$ & $3 \%$ & $\begin{array}{l}\text { - No new wind or PV generation installations after the } \\
\text { year } 2012 \text {. } \\
\text { - Minimal transmission expansion. }\end{array}$ \\
\hline $\begin{array}{l}\text { RTx10 } \\
\text { (Regional Transmission } \\
\text { and } 10 \% \text { VG) }\end{array}$ & $12 \%$ & $0.25 \%$ & $12 \%$ & $\begin{array}{l}\text { - An approximately } 10 \% \text { VG penetration as reflected in } \\
\text { state RPS and interconnection queues as of } 2012 .{ }^{4} \\
\text { - Intra-regional transmission expansion. }\end{array}$ \\
\hline $\begin{array}{l}\text { RTx30 } \\
\text { (Regional Transmission } \\
\text { and } 30 \% \text { VG) }\end{array}$ & $20 \%$ & $10 \%$ & $30 \%$ & $\begin{array}{l}\text { - Approximately } 30 \% \text { combined VG, with targets met } \\
\text { using within-region wind and PV resources. } \\
\text { - Identical transmission expansion to RTx } 10 \text {. }\end{array}$ \\
\hline $\begin{array}{l}\text { ITx30 } \\
\text { (Inter-regional } \\
\text { transmission and } 30 \% \\
\text { VG) }\end{array}$ & $25 \%$ & $5 \%$ & $30 \%$ & $\begin{array}{l}\text { - Approximately } 30 \% \text { combined VG, with an emphasis } \\
\text { on the best wind and PV resources in the U.S. El. } \\
\text { - Interregional transmission expansion with large high- } \\
\text { voltage direct current (HVDC) lines. }\end{array}$ \\
\hline
\end{tabular}

Moving from the four study scenarios to detailed operational impacts analysis required several additional steps. In order to implement the overarching guidance of the TRC, we:

1. Developed and benchmarked a model for the Eastern Interconnection, representing the year 2010.

2. Conducted a generation expansion with NREL's ReEDS model to implement the qualitative scenario requirements from the TRC (see Section 3).

3. Built scenario datasets for the forecast year 2026. This high-resolution dataset included 5minute load, wind, and PV generation and forecasts, regional reserve requirements and transmission expansions from the EIPC (see Section 4).

4. Used EI data and capacity expansion modeling results as inputs for operations modeling in the UC\&ED model, PLEXOS, to simulate future power system operations (model details are described in Section 4).

\footnotetext{
${ }^{3}$ Values displayed in this table are target penetration levels. After simulation, the annual penetration for the LowVG, RTx10, RTx30, and ITx30 were $3 \%, 11 \%, 28 \%$, and $29 \%$, respectively.

${ }^{4}$ For simplicity in naming conventions, the scenario acronym uses the number 10 instead of the actual 12.
} 
5. Analyzed the operations modeling results to identify a range of potential impacts for each study scenario.

The TRC continued its advisory role through the development of the data, tools, and inputs for the operations modeling. 


\section{Generation Expansion and Retirements}

ERGIS is an operational impact study, and in order to analyze the impact of VG on power system operations, an internally consistent methodology was necessary to determine the location, type and size of generation resources. The NREL ReEDS model was used to determine the generation fleet location and size for each scenario.

ReEDS is a capacity expansion model. It optimizes generation and transmission capacity in 2year time increments between 2010 and 2050 to determine the type and location of conventional and renewable resource development as well as a transmission infrastructure expansion for those installations. ${ }^{5}$ The model is designed to satisfy regional demand requirements and maintain grid system adequacy at least cost. The model also considers technology, resource, and policy constraints, including RPS. The model represents the contiguous United States with high spatial resolution, comprising 134 model balancing areas and 356 regions with distinct renewable power resource characteristics (of which 92 balancing areas and 248 renewable power regions are in the EI and the remainder are in WECC or ERCOT). This high spatial resolution is designed to represent the relative value of geographically and temporally constrained renewable power resources. ${ }^{6}$

The ReEDS modeling was completed before two important developments relevant to modeling the EI. The first development was changes to the boundaries of the MISO and SPP market regions, and the second was the EPA's announcement of the proposed Clean Power Plan. Therefore the ReEDS model assumed market regions as shown in Figure 3, and did not include any representation of the EPA's Clean Power Plan.

Because ERGIS adopted the transmission expansion from EIPC and because the study year is 2026, the only ReEDS results that were imported into the PLEXOS model were the cumulative generator retirements and expansion through 2026.

\footnotetext{
${ }^{5}$ The ReEDS transmission expansion was not used in the study scenarios in favor of the industry-vetted EIPC Phase II transmission expansions.

${ }^{6}$ The ReEDS model has been applied to an array of analyses, including U.S. DOE (2008), NREL (2012), U.S. DOE (2012), and U.S. DOE (2015). Documentation of the model is available in Short et al. (2011).
} 


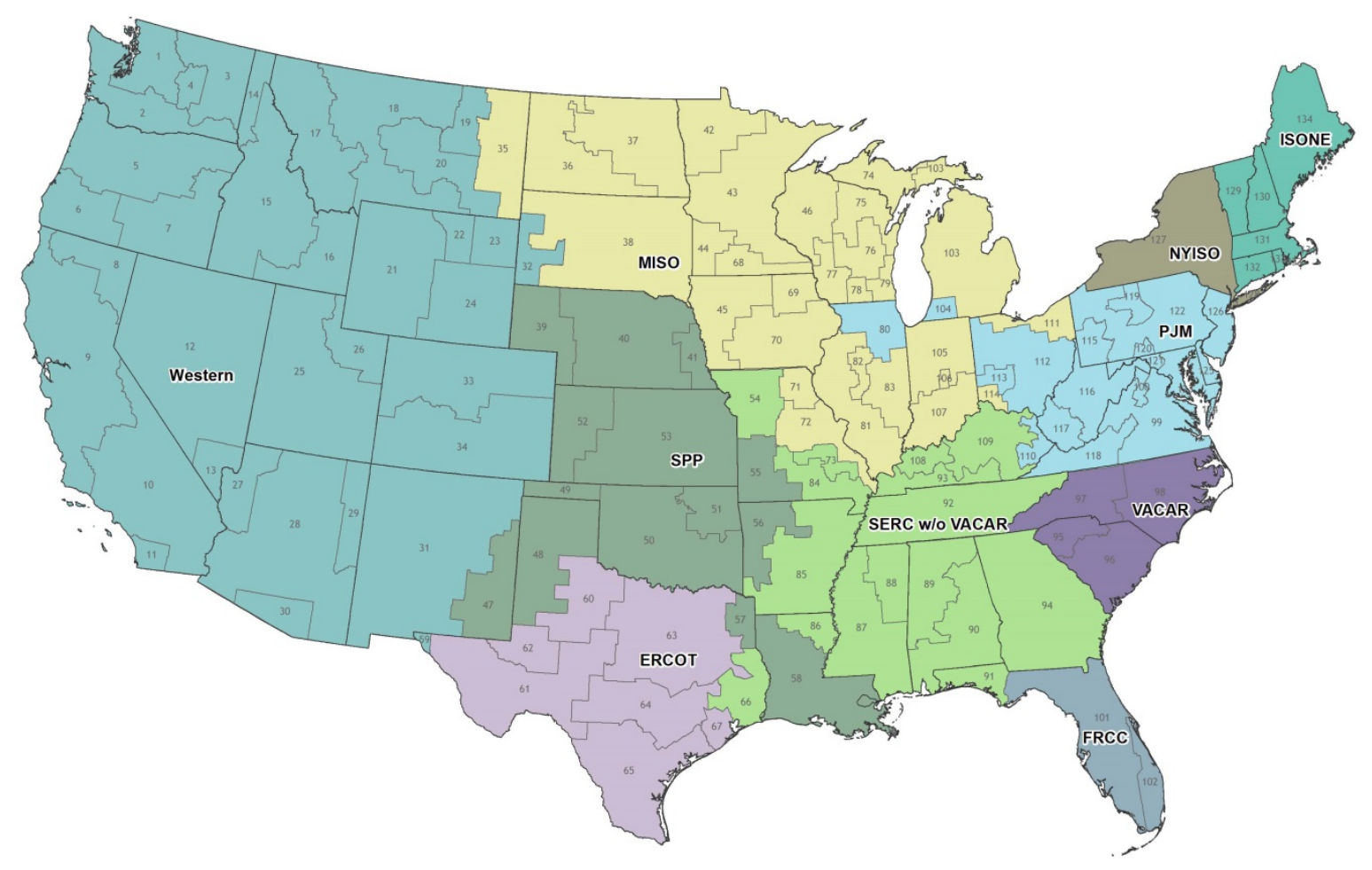

Figure 3. ReEDS regions as represented in ERGIS

\subsection{Modeling the Study Scenarios in ReEDS}

ReEDS was configured to meet wind and PV generation targets prescribed for the four study scenarios for the year 2026. The following paragraphs describe the study scenarios as modeled in ReEDS.

The LowVG scenario assumed no new builds of wind or PV after 2012, so only existing wind and PV as of 2012 was included in the 2026 generation fleet. As such, wind provides about 3\% of annual U.S. EI generation.

The RTx10 scenario assumed the state RPS requirements and interconnection queues as of 2010 were built out completely. The EIPC Phase II study developed requirements to reflect the 2010 existing state RPS requirements and interconnection queues (CRA International Inc. 2011), and the ERGIS study team elected to adopt these requirements for consistency.

The RTx30 scenario was designed to achieve 30\% wind and PV penetration in each of the EI geographic regions shown in Figure 3. The target mix of renewables varies between the regions due to differences in the quality of wind and PV resources. As such, regional exceptions were made for the SERC Reliability Corporation (SERC) and Florida Reliability Coordinating Council (FRCC) regions. Two adjustments to the capacity expansion constraints were made for SERC because of the small amount of wind resource data for SERC contained in the Eastern 
Wind Dataset. ${ }^{7}$ First, the historical Virginia-Carolinas sub-region of SERC (VACAR) was separated due to the availability of offshore wind resources on the Virginia-Carolina coast. Second, a requirement that $15 \%$ of the non-VACAR SERC load be met with wind imported from SPP was created. This import of wind from SPP was previously studied by the Electric Power Research Institute (EPRI) (EPRI 2011). The remainder of the SERC VG requirement was met with local PV resources. Similarly, because FRCC does not have wind resources in the NREL Eastern Wind Dataset, that region was allowed to fulfill its total regional 30\% requirement with PV. The RPS and interconnection queue requirements from RTx10 are also included in the RTx30. The final division of VG penetration was $20 \%$ wind and $10 \% \mathrm{PV}$ for the U.S. EI.

The ITx30 scenario was designed to achieve 30\% penetration with fewer constraints on wind and PV location, allowing higher-quality resources to be developed and delivered to other regions. The penetration for wind was 25\%, with 20\% land-based and 5\% offshore, while the PV penetration target was 5\%. Regional PV targets were set so that distributed PV adoption (which is not represented in ReEDS) could be represented. The distributed PV requirements are the same as for RTx30. In general, the wind resources in MISO and SPP are expanded in the ITx30 scenario because of the high quality of those resources and the lower capital costs of wind compared to PV.

\subsection{Thermal Fleet Retirement Assumptions and Results}

Retirements in ReEDS are determined in three ways: announced retirements, maximum generator lifetimes, and minimum utilization factors. Each of these retirement mechanisms are described in more detail below.

Announced retirements are provided as inputs to ReEDS and dominate the capacity reductions between 2010 and 2016. Assumptions regarding coal retirements were obtained from Saha $(2013)^{8}$ and contain about $45 \mathrm{GW}$ of coal capacity retirements in the U.S. EI. Announced retirements for other generating technologies were taken from ABB (2013) and contain about 30 $\mathrm{GW}$ of natural gas capacity retirements.

In addition to announced retirements, generators are assumed to have maximum economic lifetimes. Fossil fuel plant ages are derived from data reported by ABB (2013), ${ }^{9}$ and assumed lifetimes for these generators are shown in Table 3.

\footnotetext{
${ }^{7}$ The Eastern Wind Dataset is based on wind resource maps for 80 meters above ground, and did not include the potential use of taller wind turbines to capture wind resources higher above the ground. Since 2011, more than 1,000 turbines have been installed in the U.S. at 100 meter or greater hub heights, and there has been increased interest in the use of even taller turbines to take advantage of wind resources that are available at higher hub heights in regions such as the Southeast U.S. (DOE 2015).

${ }^{8}$ Due to ReEDS geospatial requirements, these data reflect announced retirements only (e.g., Saha 2013). Other estimated retirements (e.g., Patrylak et al. 2013; Brattle Group 2012; DiOrio et al. 2011) lack sufficient geospatial and temporal resolution to be incorporated into ReEDS but are addressed to a degree by overlaps with Saha (2013), and by the age-based and plant-utilization-based retirements.

${ }^{9} \mathrm{~A}$ single service life extension period was selected for nuclear units given the significant uncertainty in current nuclear plant lifetimes.
} 
Table 3. Maximum Economic Lifetime of Thermal Plants in ReEDS

\begin{tabular}{|l|c|}
\hline Plant Type & Assumed Economic Lifetime (Years) \\
\hline Coal < 100 MW & 65 \\
\hline Coal > 100 MW & 75 \\
\hline Natural Gas and Oil & 55 \\
\hline Nuclear & 60 \\
\hline
\end{tabular}

The final retirement mechanism in ReEDS is underutilization. Utilization-based retirements only apply to coal generators and are a proxy for economic-based considerations (i.e., insufficient revenue to support forward operation costs). Underutilization retirements are represented as capacity factor thresholds that trigger generation capacity to be retired. ReEDS' capacity factor thresholds increase over time: in 2020, the generation capacity factor threshold is $1 \%$, and the threshold increases by $5 \%$ per model solve year (i.e., every two years). This means the minimum capacity factor thresholds were $1 \%, 6 \%, 11 \%$, and $16 \%$ in $2020,2022,2024$, and 2026 , respectively.

Table 4 shows the retirements results from the ReEDS model for the LowVG scenario for coal, gas, and nuclear generators. In all scenarios, announced retirements result in about $45 \mathrm{GW}$ of coal retirements and $30 \mathrm{GW}$ of gas retirements by 2016. Age-based retirements cause an additional $16 \mathrm{GW}$ of coal retirements and $30 \mathrm{GW}$ of gas retirements by 2026 . Announced retirements of nuclear units in the U.S. EI (Kewaunee, Crystal River, Vermont Yankee, and Oyster Creek) total about 2.6 GW by 2020 .

Table 4. U.S. EI Thermal Capacity Retired by ReEDS LowVG by 2026

\begin{tabular}{|l|c|}
\hline Generator Type & Retired Capacity (GW) \\
\hline Nuclear & 2.6 \\
\hline Coal & 60.6 \\
\hline Gas & 59.7 \\
\hline
\end{tabular}

To minimize the number of differences between the scenarios, the thermal fleet changes from the LowVG were applied to the other scenarios, and only the VG fleet changes from the ReEDS analysis of the other scenarios were utilized in ERGIS. The LowVG had the lowest amount of $\mathrm{VG}$, so the thermal fleet is larger than is necessary for the other scenarios. Table 5 shows the change in installed capacity determined by the ReEDS analysis for each of the ERGIS scenarios from 2010 through 2026 . The additional installed thermal capacity may result in a system with more thermal generating capability than would be expected under market conditions. 
Table 5. Change in Installed Capacity of the U.S. EI Fleet from 2010 to 2026

\begin{tabular}{|l|r|r|r|r|}
\hline \multirow{2}{*}{$\begin{array}{l}\text { Generator } \\
\text { Type }\end{array}$} & \multicolumn{4}{|c|}{ Change in Installed Capacity (GW) } \\
\hline Nuclear & LowVG & RTx10 & RTx30 & ITx30 \\
\hline Coal & 0.9 & 0.9 & 0.9 & 0.9 \\
\hline Gas & -50.5 & -50.5 & -50.5 & -50.5 \\
\hline PV & 88.1 & 88.1 & 88.1 & 88.1 \\
\hline Wind & 0.7 & 5.1 & 194.1 & 133.9 \\
\hline & 10.6 & 150.8 & 209.7 & 261.0 \\
\hline
\end{tabular}




\section{Input Data}

To model the grid realistically, the UC\&ED model required extensive input datasets, which include generator and transmission constraints and other elements to capture the challenges of operating the electric grid. We assembled the necessary inputs, such as generator characteristics, transmission topology, and wind and PV generation profiles, under the guidance and review of the TRC. This section gives an overview of the data and tools used to build the UC\&ED model.

\subsection{Eastern Interconnection Model Development}

Studying future generation scenarios requires a model that has been vetted by industry and benchmarked against historical data. In this section, we detail the input assumptions made for a model of the EI in the year 2010. We then compare simulation results to historical observations from the same year. This process resulted in a suitable starting point for evaluating operations in the 2026 study year.

\subsubsection{Conventional Fleet Assumptions}

We assembled data and assumptions for the conventional generation (non-VG) fleet from a variety of sources. Each of these assumptions is described in this section.

The non-VG generator maximum capacities, locations, and generator technologies were provided by Energy Exemplar, the vendor of PLEXOS.

All units except nuclear units were assumed to be available for economic commitment and dispatch any time they were not on a planned or forced outage. Due to lack of information, no must-run, self-scheduling, or bilateral contract constraints were included. Nuclear units were assumed to operate at their maximum capacity at all times that they were not on a planned or forced outage. This is a conservative assumption, as nuclear plants in several countries and the Pacific Northwest have decades of successful flexible plant operation (EPRI 2014).

Full-load heat rates (i.e. heat rate at maximum operating capacity) for coal-fired and gas-fired generators were assigned to each generator in these categories so that the fleet-wide distribution of the heat rates matched the fleet-wide distribution of full-load heat rates found in a previous analysis of EPA Continuous Emissions Monitoring Systems (CEMS) data (Lew et al. 2012). Figure 4 shows the distribution of the full-load heat rates for the coal-fired and gas-fired generators. These heat rates were used for both the 2010 benchmarking of the EI model and the 2026 study scenarios. 

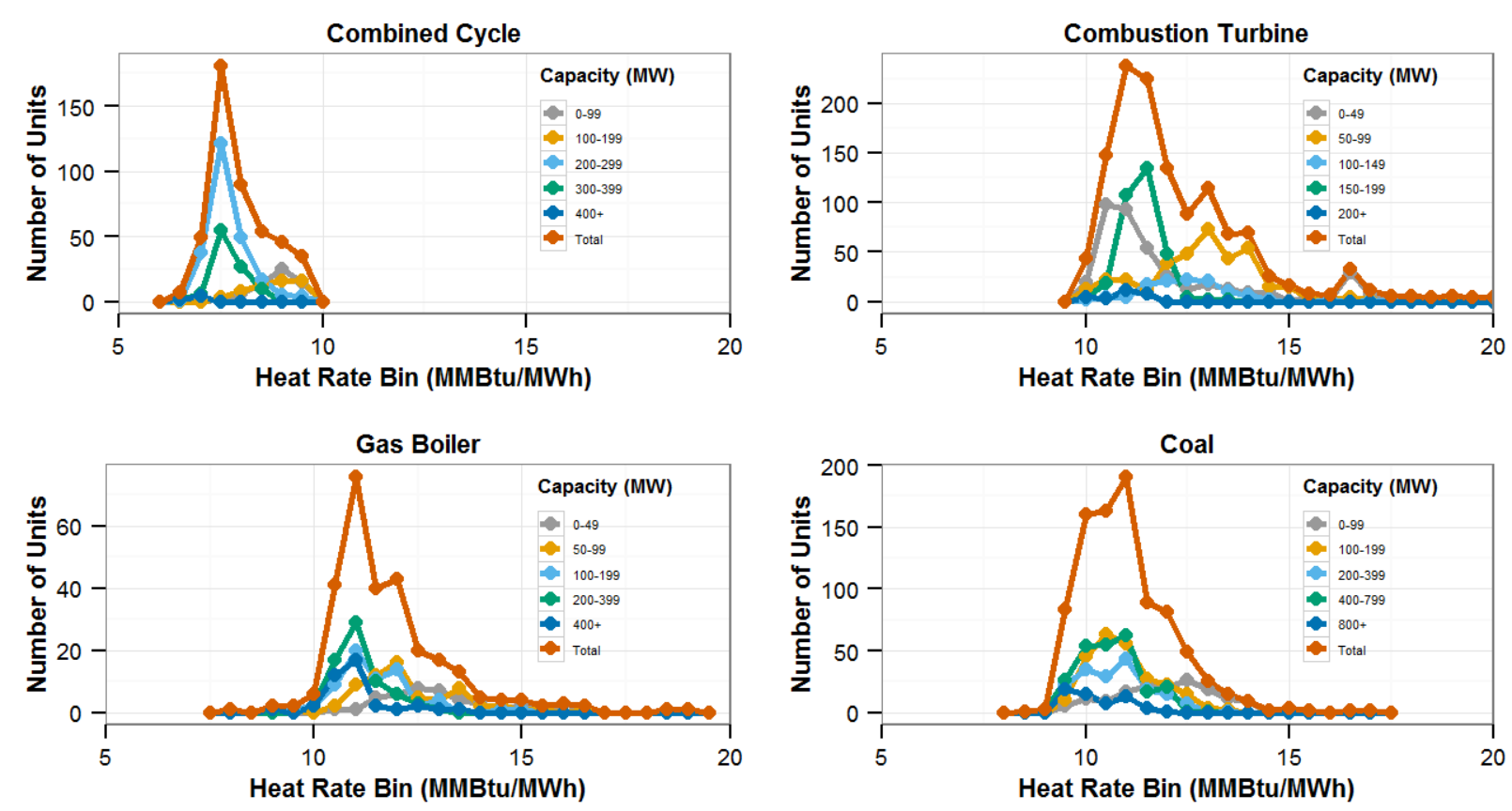

Figure 4. Distribution of full-load heat rates for coal-fired and gas-fired generators

Table 6 summarizes several assumptions about thermal generator properties, including minimum generation level, part-load heat rates (i.e. heat rates at operating points below maximum capacity), minimum up and down times, ramp rates, startup costs, and variable operations and maintenance costs. ${ }^{10}$

Several generator property assumptions were adopted from the EIPC Phase II (Eastern Interconnection Planning Collaborative 2102) with minor revisions. We adopted, without modification, part-load efficiency deratings and minimum up and down times. We modified the EIPC Phase II assumptions for the number of marginal heat rate blocks and the minimum generation level for CT generators. To reduce runtimes, a single marginal heat rate between the minimum and maximum generation level was used instead of two or three marginal heat rate blocks (part-load efficiency deratings were represented using non-zero no-load fuel consumption). In addition, to enforce proper commitment status of CT generators, the minimum generation level for CT generators was set at $60 \%$ of the CT's maximum capacity instead of the EIPC's assumption of $0 \%$.

Ramp rates were taken from Black and Veatch (2012), and startup costs and variable operations and maintenance (VO\&M) costs were taken from Kumar et al. (2012).

Forced outage rates, maintenance outage rates, and mean repair times for nuclear, coal, gas, oil, and hydroelectric generators were taken from North American Electric Reliability Corporation (NERC) Generator Availability Data System (GADS) data (GADS 2015). GADS provides detailed information by unit maximum capacity for most of the major generator types, and these data were applied to the ERGIS UC\&ED model.

\footnotetext{
${ }^{10} \mathrm{CT}$ and gas boiler technologies have different properties (as reported in the table) but because they have similar marginal costs they behave similarly in the model. For ease of reporting, the two technologies are combined into a single category referred to collectively as CT/Gas boilers.
} 
Table 6. Selected Assumptions for Thermal Generators

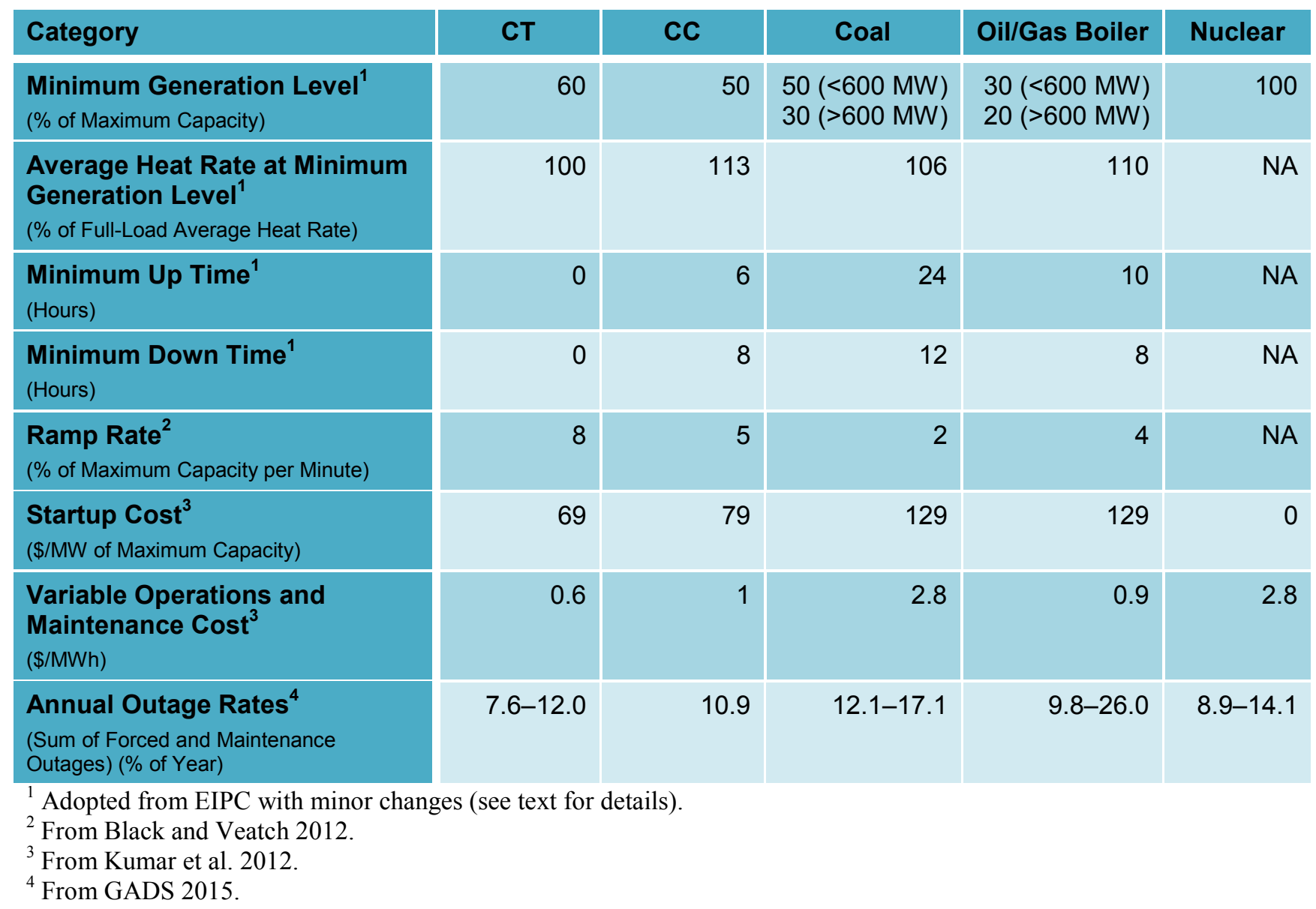

Fuel prices for the study were taken from EIA Annual Energy Outlook (AEO) 2014 reference case projections for 2026 (U.S. Energy Information Administration 2013). Monthly variation based on historical patterns was added to the natural gas prices. Prices for each of the AEO census regions for coal and natural gas are shown in Figure 5 and Figure 6, respectively. The unweighted average price of gas is $\$ 5.92 / \mathrm{MMBtu}$ and coal is $\$ 2.90 / \mathrm{MMBtu}$.

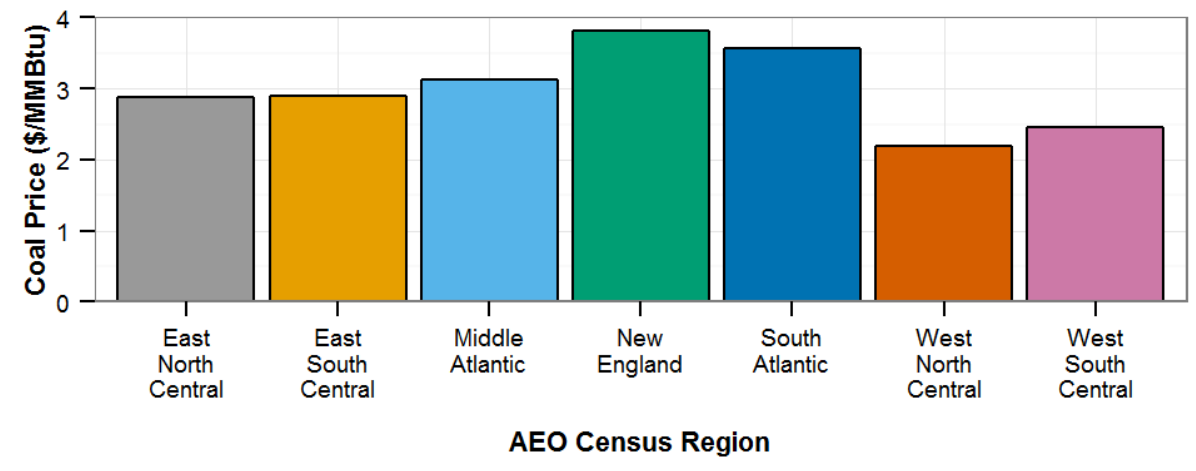

Figure 5. Yearly coal prices used in the UC\&ED model. 


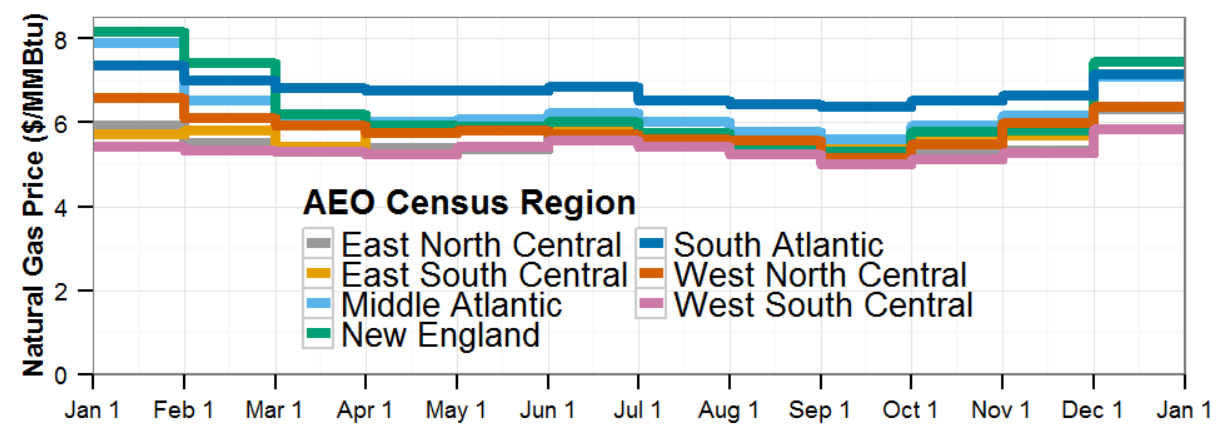

Figure 6. Monthly natural gas prices used in the UC\&ED model.

Modeling hydroelectric (hydro) resources in a UC\&ED model is typically more complex than modeling traditional thermal generators. This is because of additional constraints around water usage and the choice of when to use the (often limited) water. Most rivers and reservoirs are managed with multiple objectives beyond power production, such as recreation, flood control, ecological health, and water supply. Most of these constraints are not captured explicitly in large-scale power systems operations studies, including this one, although we made assumptions about availability based on historic generation profiles, reservoir levels, and river flows.

Hydro is approximately $10 \%$ of total installed capacity in the present-day EI and is much higher in some regions. ${ }^{11}$ This makes the modeling of hydro constraints important to accurately representing operations. NREL used historical data from annual reports of utilities, operating organizations, and the U.S. EIA to create limits on the amount of energy that each hydro generator can generate at different times of the year. NREL used these limits within PLEXOS to constrain the amount of energy a hydro unit was able to produce over a certain time period. The hydro units were able to dispatch energy economically within these constraints. In addition, all hydro generators were assigned a minimum generation level of $20 \%$, based on guidance from the TRC. This constraint is intended to represent non-power production constraints. NREL developed different types of hydro generation limits based on historical data. For the Southeastern Power Administration (SEPA), Southwestern Power Administration (SWPA), and certain U.S. Army Corps of Engineers (USACE) facilities within the U.S. EI, NREL obtained historical daily or weekly generation data (USACE 2015a and 2015b, TVA 2015). For other U.S. hydro facilities within the U.S. EI, NREL obtained actual monthly historical generation from the EIA (EIA 2015c). For Canadian hydro facilities, NREL obtained historical generation from Canadian sources (OPG 2015, Manitoba Hydro 2014, SaskPower 2012, IESO 2015, HydroQuébec 2012). Each of these types of generation limits is described in the following paragraphs.

NREL obtained the most detailed historical generation data from SEPA, SWPA, and USACE. This data included actual historical generation totals down to daily resolution. Interviews with representatives from these organizations indicated that water availability was typically budgeted

${ }^{11} \underline{\text { www.eia.gov/cneaf/electricity/page/eia860.html }}$,
$\underline{\text { http://www.hydroquebec.com/publications/en/docs/annual-report/annual-report-2014.pdf }}$ 
on a weekly basis. This allowed generation to be scheduled anytime within that week. Therefore, NREL created weekly generation constraints for these facilities from the historical weekly generation totals.

One example facility is USACE's Wolf Creek Dam in Kentucky. The facility's 2006 historical data indicates that its annual capacity factor was about $33 \%$; but its weekly capacity factor varied from a minimum of about $7 \%$ to a maximum of about $64 \%$. NREL created weekly energy constraints from the historical data, as shown in Figure 7.

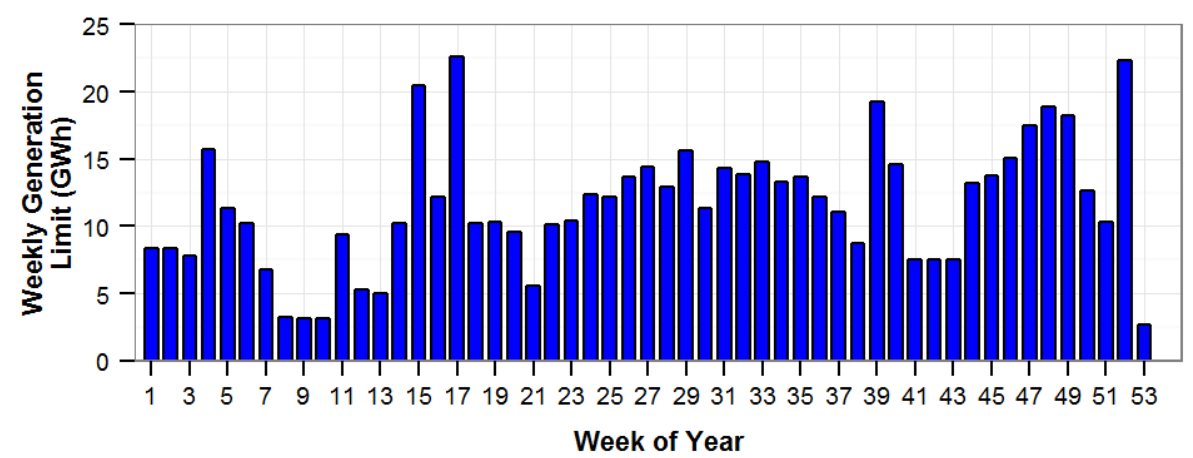

Figure 7. Example weekly hydro generation constraints for SEPA, SWPA, and USACE facilities

NREL obtained historical generation data for other U.S. hydro facilities from EIA-920 data. These data consist of historical monthly generation totals. NREL used the monthly generation totals for 2006 to create monthly generation limits for the non-SEPA, non-SWPA, non-USACE hydro facilities in the U.S. EI.

Canadian hydro generating resources are significant. In order to capture the constraints of hydro generators in Manitoba, New Brunswick, and Ontario, NREL found historical annual generation data for individual generators and then paired that data with historical stream flow data by month to create monthly generation limits. For hydro generators in Québec and Saskatchewan, NREL estimated annual generation for each unit based on reported total system generation and individual unit capacity and then paired the estimated annual generation data to historical stream flow data by month to create monthly generation limits. Figure 8 illustrates how annual generation was distributed into monthly limits in proportion to the monthly streamflow. 

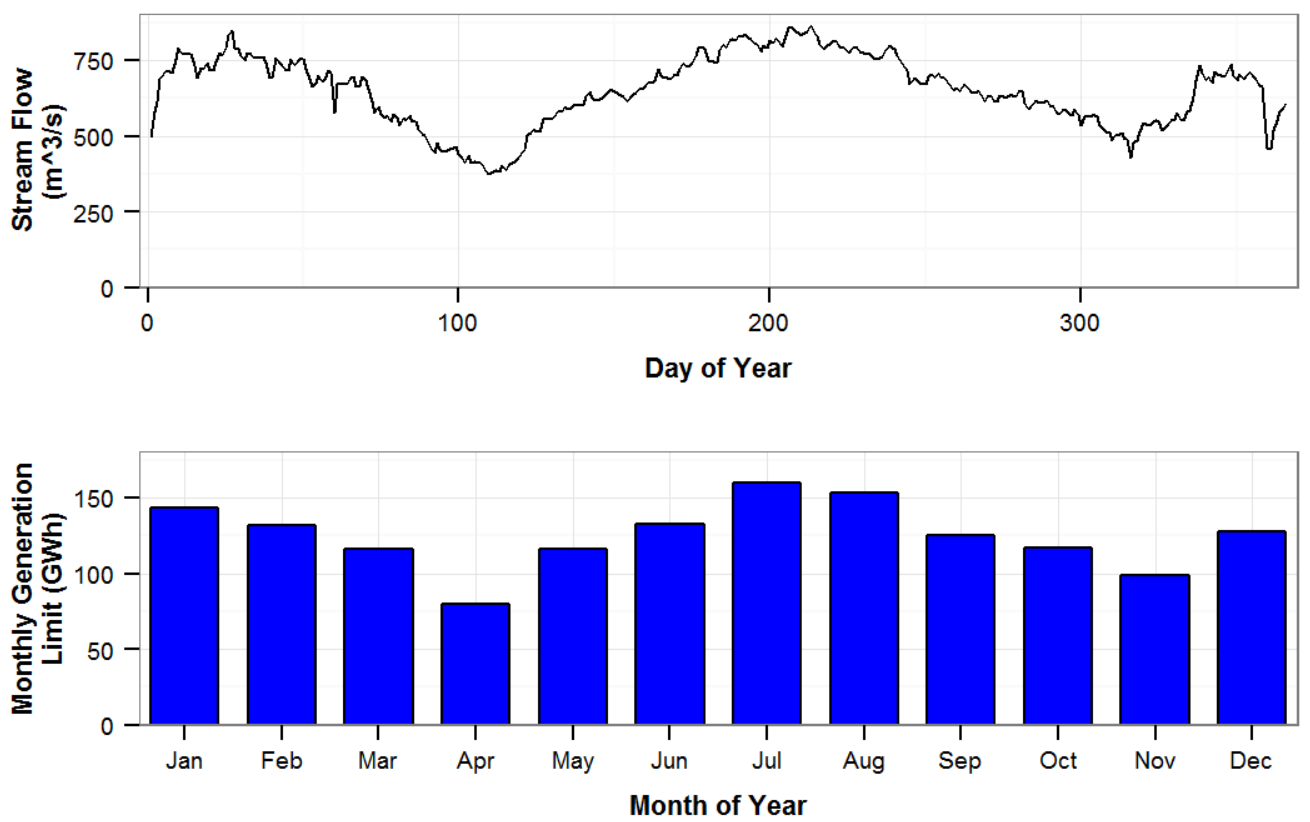

Figure 8. Example monthly limits for Canadian hydro generators

\subsubsection{System}

As the ERGIS project began in 2012, the most recent year for which a full set of load, fuel price, and weather data was available was 2010 , so 2010 was selected as the year to benchmark model results against actual historical data.

Because the benchmarking model was intended to resemble 2010, the 2010 boundaries of all Regional Transmission Operators, Independent System Operators (collectively, RTO/ISO) and NERC reliability regions were used. The 2010 study regions were FRCC, Independent System Operator of New England (ISO-NE), MISO, New York Independent System Operator (NYISO), PJM Interconnection (PJM), SPP and SERC. The base transmission network of the entire EI was obtained from the Eastern Interconnection Reliability Assessment Group's (ERAG's)

Multiregional Modeling Working Group and imported into the UC\&ED model. This network contains approximately 60,000 transmission buses, 50,000 transmission lines, and 20,000 transformers. The full network was imported into the UC\&ED model without network reduction.

\subsubsection{Benchmarking}

Because the EI model provided by the vendor of PLEXOS had not been vetted nor evaluated prior to ERGIS, we performed a benchmarking exercise to ensure that the modeled results would be comparable to the real-world system. The goal in this exercise was to ensure that the regional load levels, generation capacities and actual generation, and regional interchange patterns were reasonable.

The EI model was set up with 2010 conditions for load, generator and transmission capacities, and fuel prices, and the results were compared to actual 2010 data reported by the EIA (EIA 2015c). Because the benchmarking exercise was only focused on high-level characteristics, such as generation by generator type and net interchange between regions, the benchmarking model 
employed a 2-hour resolution day-ahead model and no real-time model. The benchmark modeling also employed a simplified representation of reserves, with a single reserves requirement of $2.5 \%$ of load required at all times. These simplifications allowed significantly faster runtimes without substantial difference in the higher-level outputs that were compared.

Figure 9 compares the modeled and actual generation by fuel type for each of the regions as given by the benchmarking process. The model results show that generation by region and generator type is in general agreement with the actual 2010 data. The differences that remain between the model results and actual 2010 data are likely due to differences in regional definitions, imperfect knowledge of actual generator costs and availability in the model, differences in transmission constraints, and non-economic dispatch constraints in actual operation (such as bilateral agreements and must-run units) that are not included in the model.
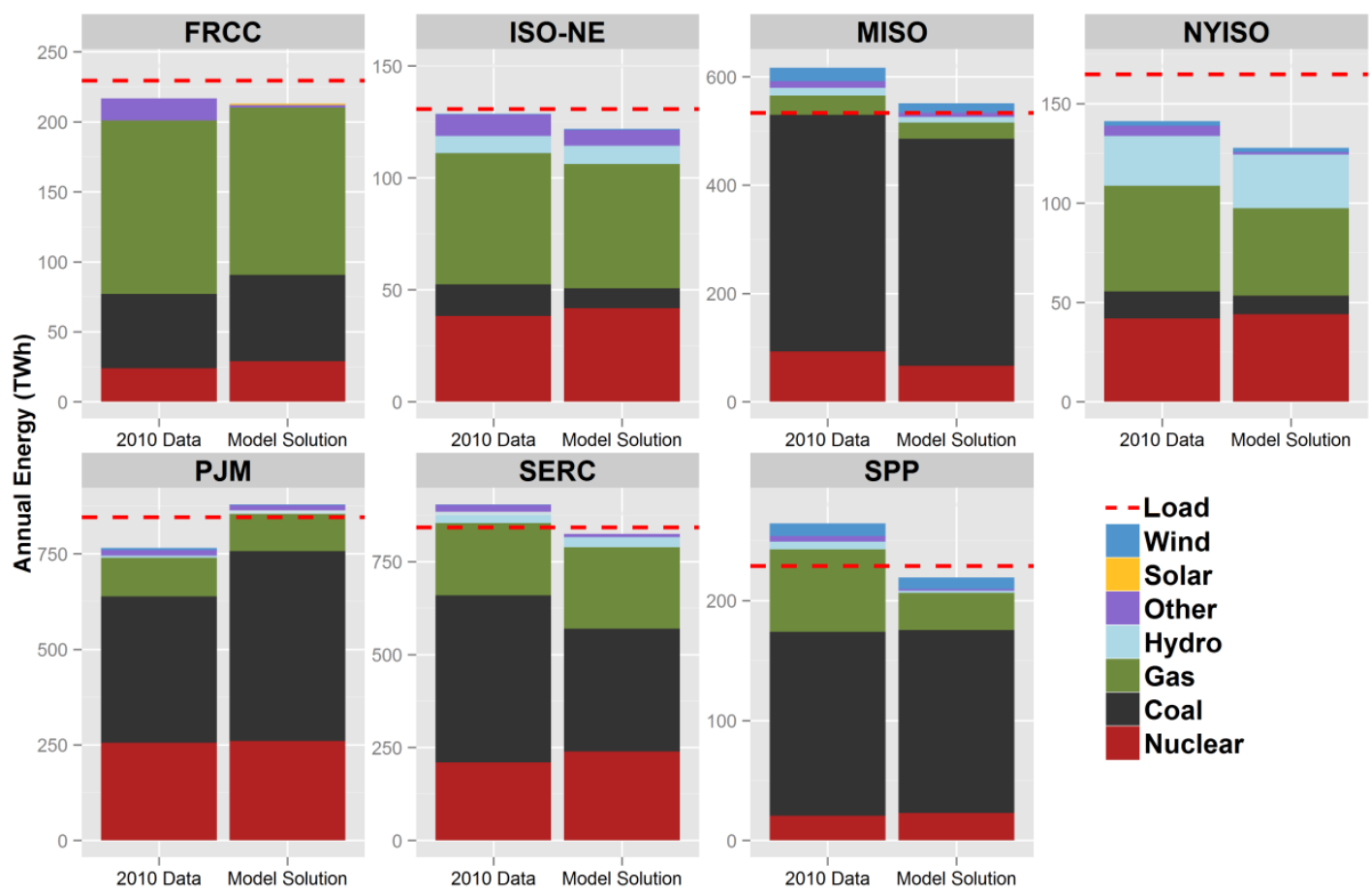

Figure 9. Generation by fuel type and region in the benchmarking exercise compared to actual 2010 data reported by the EIA

Figure 10 shows the annual net interchange between regions as given by the benchmarking process. This result was compared to available EIA data (EIA 2011b) and is in general agreement on both direction and magnitude of the net flows. 


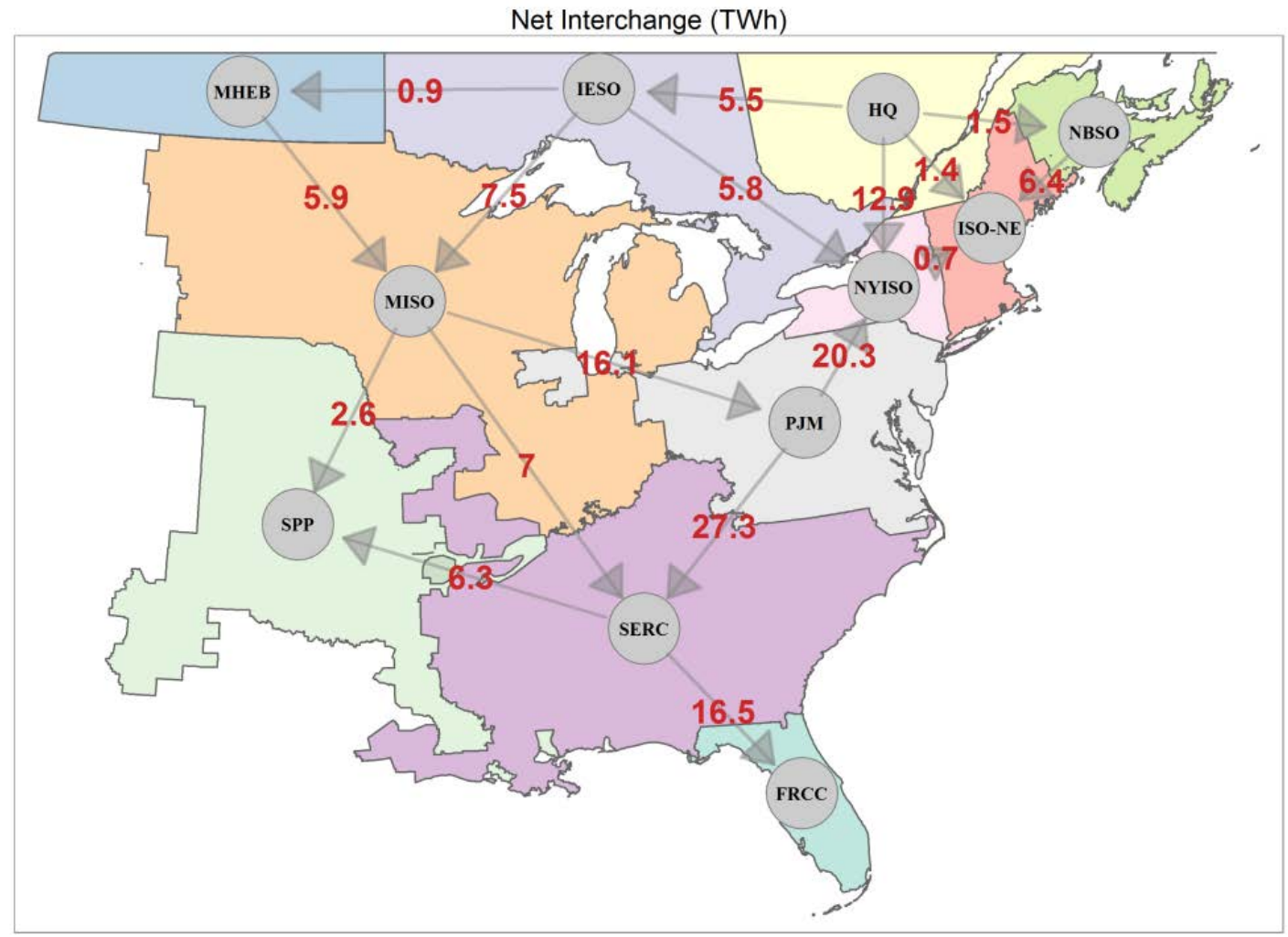

Figure 10. Map showing the net interchange patterns for the 2010 benchmarking exercise

\subsection{Wind, PV, and Load Data Creation}

In order to study power system operations at a 5-minute level, it was necessary to create datasets of sufficient resolution and fidelity to simulate the operation of wind and PV generation and demand. Simulated wind data was previously created for EWITS, but that data was not of a 5minute resolution. A comprehensive PV dataset for the U.S. EI did not exist prior to ERGIS and was created for this study. Load data shares the same meteorological year (2006) as wind and PV resources and was downscaled from hourly data. In this section, NREL describes the process for creating these datasets and how simulated wind and PV resources were sited within the model.

\subsubsection{Variable Generation Fleet Assumptions}

A large amount of wind and PV data was needed for this study. This section describes the data used, including synthesis of the data where existing data was not available. This includes the methodology for selecting individual theoretical sites for wind and PV plants for the ERGIS scenarios to match the ReEDS wind and PV generation results.

\subsubsection{Wind Resource Data}

ERGIS used NREL's Eastern Wind Dataset for time-series wind data. The Eastern Wind Dataset consists of wind power forecasts and simulated power output for the meteorological years 2004 through 2006, although only the 2006 data were used in the UC\&ED simulations to match the load and PV data years. The Eastern Wind Dataset is available to the public, for free, and can be 
obtained online. ${ }^{12}$ The data were originally developed by AWS TruePower for EWITS (Enernex Corporation 2011) and updated in Pennock (2012). The dataset was developed for EWITS, and is intended for use by energy professionals such as transmission planners, utility planners, project developers, and university researchers who perform wind integration studies and estimate power production from hypothetical wind plants. The model is initialized with input from the National Centers for Environmental Prediction-National Center for Atmospheric Research Global Reanalysis dataset and assimilates both surface and rawinsonde data. The model used a nested grid scheme, with an output resolution of $2 \mathrm{~km}$.

The Eastern Wind Dataset contains data at a 10-minute resolution for 1,326 simulated wind plants and includes next-day, six-hour ahead, and four-hour ahead forecasts for each plant as well as actual simulated generation. Each set of forecasts was synthesized by running a statistical forecast synthesis tool written by AWS Truepower called SynForecast. This tool uses actual forecasts and observed plant output to develop a set of transition probabilities that are then applied stepping forward in time from a random starting point in a process known as a Markov chain. A fast Fourier transform-based method was used to synthesize 5-minute data from the original 10-minute data (Figure 11).

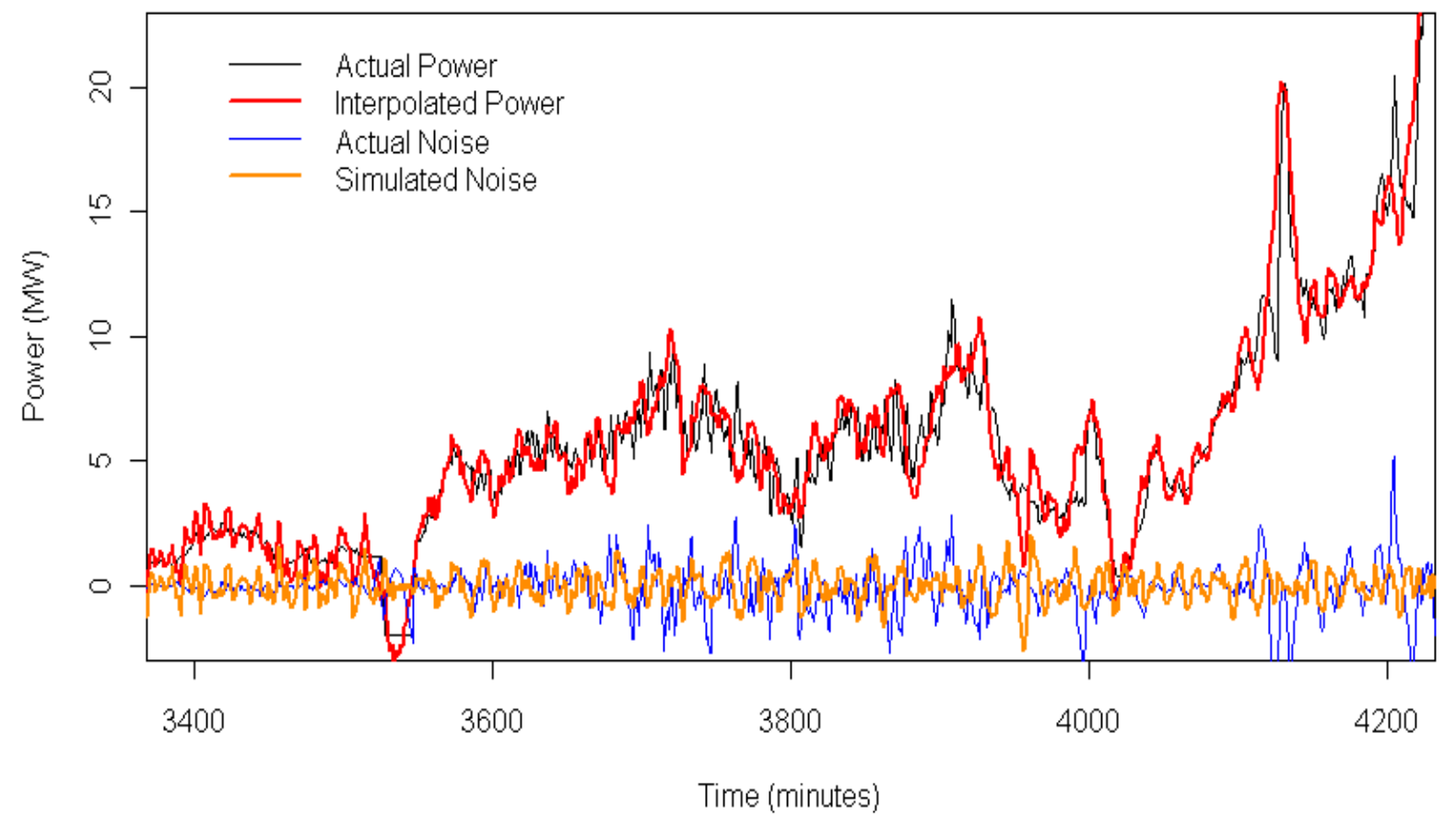

Figure 11. Graph showing 5-minute wind generation data that was created from adding simulated noise to the 10-minute wind generation data

\footnotetext{
${ }^{12}$ The Eastern Wind Dataset for years 2004-2006 is available at http://www.nrel.gov/electricity/transmission/eastern wind methodology.html. Under the Wind Integration National Dataset project, a new dataset for the years 2007-2013 was created Draxl et al (2015). This data was not available at the beginning of the ERGIS project, but is now available for free online:

http://www.nrel.gov/electricity/transmission/wind toolkit.html.
} 


\subsubsection{PV Resource Data}

Hypothetical PV time-series data was created for this project using the sub-hour irradiance algorithm developed by Hummon et al. (2012). This dataset was created under the Solar Integration National Dataset (SIND) Toolkit and enables researchers to perform regional PV generation integration studies by providing modeled, coherent sub-hour PV power data, information, and tools. The data are intended for use by energy professionals such as transmission planners, utility planners, project developers, and university researchers who perform PV integration studies and need to estimate power production from hypothetical PV plants. ${ }^{13}$ The sub-hour irradiance algorithm produces statistically probable values of irradiance with a temporal resolution of one minute from satellite-image-derived irradiance data with a temporal resolution of sixty minutes. This algorithm was developed under the Western Wind and Solar Integration Study Phase II (WWSIS-2). The final dataset includes 5-minute data for approximately 4,000 simulated PV plants in the Eastern Interconnection. Both utility scale PV and distributed rooftop PV were developed in this dataset. Distributed PV sites have a fixed tilt of 15 degrees, and are oriented south at 180 degrees. Half of all utility scale PV is assumed to have a fixed tilt of 15 degrees and oriented south at 180 degrees, and the other half is assumed to be tracking. Both distributed and utility-scale PV are assumed to be fully controllable (curtailable) by the system operator.

The simulation of 1-minute irradiance values draws on three sets of statistics: spatial variability of the clearness index (from gridded hourly satellite data), distribution of 1-minute clearness index ramps (from ground-based measured stations), and distributions of the duration of a persistent 1-minute clearness index (from ground-based measured stations). The algorithm was built from 26 year-long, ground-based, 1-minute measured irradiance datasets (from six locations in the western United States from the NREL Measurement and Instrumentation Data Center database), and the corresponding hourly satellite irradiance data.

The key data characteristics for the PV dataset are: (1) an appropriate number and size of power output ramps at each location, (2) appropriate coincidence of power ramps at closely clustered locations, and (3) appropriate ramps in the sum of PV power over a region. The primary goal of the algorithm was to replicate the injection of power into the transmission system from individual PV plants, such that the magnitude and duration of ramps for each plant were accurate, as well as the correlation of ramps between plants. The measure of correlation between multiple locations over multiple time scales is called coherence. The algorithm uses the spatial statistics to impose an appropriate relationship between locations. Sites that are close together share spatial data and thus have a higher likelihood of having similar time series. A statistical analysis of this data is included in Appendix A.

In order to understand the impact of uncertainty in PV generation, it was necessary to create dayahead generation forecasts for the simulated PV resources. The Weather Research and Forecasting (WRF) model (Shamarock et al. 2008) was used to mimic operational PV irradiance forecasts over the eastern United States for 1 year, January-December 2006. WRF was set up in operational mode with the aim of producing a day-ahead forecast for global horizontal irradiance, direct normal irradiance, and diffuse irradiance ready at 12 p.m. EST. The forecasts

\footnotetext{
${ }^{13}$ The SIND dataset is available for free online:

http://www.nrel.gov/electricity/transmission/solar_integration_methodology.html
} 
were thus initialized at 16 Universal Coordinated Time (UTC) each day (11 a.m. EST) and run for 38 hours to make day-ahead forecasts for midnight the next day. The simulated irradiance, temperature, and pressure values were input into PVWatts ${ }^{14}$ to create power forecasts and actuals.

\subsubsection{Load Data}

Coincident load, wind and PV data years are important for maintaining the correlation between weather-driven load, wind and PV generation patterns. Because sub-hourly load data for ERGIS did not previously exist, we needed to create it. For ERGIS, historical hourly load data from 2006 was obtained and scaled to reach projected 2026 load levels.

Multiple steps were needed to increase 2006 load profiles to 2026 forecasted load levels. First, the 2006 hourly load profiles for each region were created by summing ABB Velocity Suite (C) 2006 hourly profiles for the ABB transmission zones within each of the ERGIS sub-regions.

Scaling the 2006 load to 2026 required multiple steps because no single data source was available that could scale 2006 load to 2026.The first scale factor was calculated using 2006 to 2011 state retail load data (EIA 2015b). For this process, the increase or decrease in load from 2006 to 2011 was found for each state in the U.S. EI.

The second scale factor was calculated using EIA AEO projected growth in retail electricity sales for each of the National Energy Modeling System (NEMS) Electricity Market Module (EMM) regions (EIA 2011a). Scale factors for 2011 to 2026 were calculated as the projected 2026 load divided by the 2011 load for each EMM region.

To combine the two sets of scale factors, the state scale factors had to be aggregated to the EMM regions. This was done by mapping the states to the EMM regions and then calculating a loadweighted scale factor for each region. The two sets of scale factors were then compounded to get an aggregate for the entire 2006-2026 period for each EMM region.

Once the scales for the EMM regions were complete, the scaling factors for the individual ERGIS sub-regions were assigned. The final load scaling factors for individual regions are shown in Appendix B. Load growth for individual regions for the entire 2006-2026 period range from $1 \%$ to over $20 \%$. Table 7 summarizes the load growth for the U.S. EI between 2006 and 2026 , which increases by about $11 \%$.

Table 7. U.S. EI Load Growth Summary

\begin{tabular}{|l|l|}
\hline 2006 U.S. El Load & 2,913 TWh \\
\hline 2026 U.S. El Load & 3,238 TWh \\
\hline U.S. EI Load Growth 2006-2026 & $11.14 \%$ \\
\hline
\end{tabular}

\footnotetext{
${ }^{14}$ NREL's PVWatts Calculator estimates the energy production and cost of energy of grid-connected PV energy systems, http://pvwatts.nrel.gov/.
} 
In order to understand the impacts of variability and uncertainty at a 5-minute time resolution, a new 5-minute resolution load dataset was necessary. This dataset was derived by analyzing high resolution load data obtained from various public sources and additional datasets provided by the TRC. These data were analyzed to extract variability information using filtering techniques. That information was then processed to obtain variability statistics for each of the high resolution datasets analyzed. The statistics were then used to synthesize sub-hourly variability, which was added to the hourly load data for the study, to obtain the 5-minute data. Sub-hourly load datasets analyzed for the regions, time periods, and resolutions are identified in Table 8.

Table 8. Actual Load Datasets Used to Characterize Sub-hourly Load Variation

\begin{tabular}{|l|l|l|}
\hline Region & Period & Resolution \\
\hline ISO-NE & 2005 & 1 Minute \\
\hline MISO & 2005 & 10 Minute \\
\hline NYISO & 2005 & 10 Minute \\
\hline PJM & 2005 and 2006 & 10 Minute \\
\hline Southern Company & 2005 & 10 Minute \\
\hline SPP & 2005 and 2006 & 1 Minute \\
\hline ISO-NE & Partial 2013 & 5 Minute \\
\hline NYISO & 2012 & 5 Minute \\
\hline PJM & 2012 & 1 Minute \\
\hline SPP & 2010,2011, and 2012 & 5 Minute \\
\hline
\end{tabular}

A filter is a technique for separating characteristics of a signal. In this case NREL separated the load data into low- and high-frequency components. The low-frequency component is the load trend and the high-frequency component is the random variability around that trend. Figure 12 shows an example of the process. The blue trace is the original high-resolution data and the red trace is the output of a low-pass filter indicating the load trend. Finally, the green trace shows the variability signal. Note that the variability is on a different scale from the other two traces. 


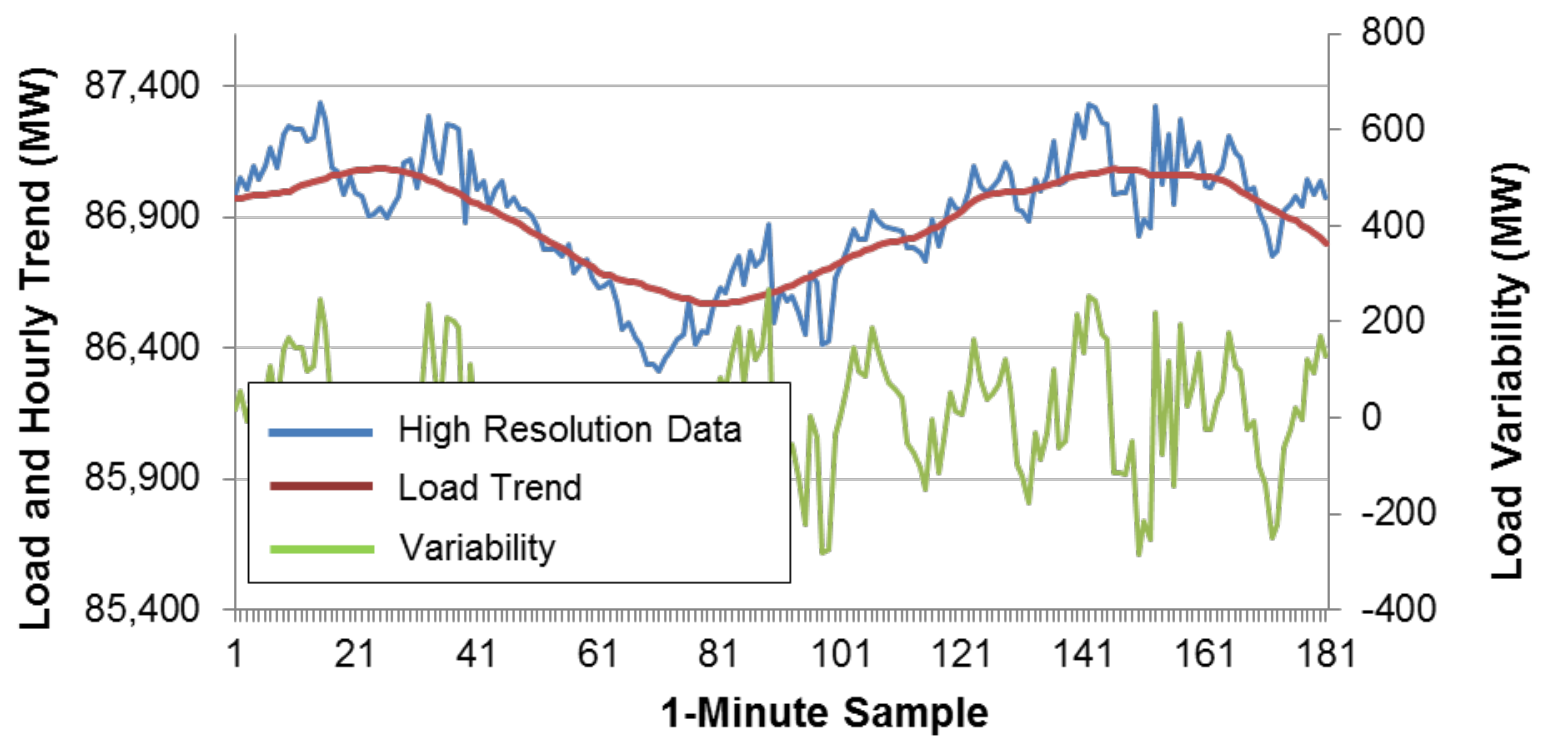

Figure 12. Filtering of high resolution data to get load variability

Several approaches were evaluated for the selection of an appropriate filter. In previous work, simple moving-average windows and other digital filters such as Butterworth $2^{\text {nd }}$ order low- and high-pass filters were evaluated, and simple moving-averages were found to be the most effective at extracting the load trend. The variability is then simply the difference between the load data and the trend.

The moving average filters were implemented such that the current period is at the center of the window with an equal number of samples coming from the future and from the past. The fact that this filter looks into the future gives it better tracking of the signal compared to the other filters tested, and therefore this filter was used to extract the trend from the load datasets.

Next, the size of the filter window size was tuned to give the best response, to filter out as much short-term variability as possible from the longer-term trend. In this case, we considered the variability to be the sub-hourly changes in the signal. Window lengths from 25 minutes to 65 minutes were evaluated to determine which window size provided the most realistic representation of sub-hourly variability.

Examples of different window sizes for December 28 from 8:30 to 10 AM in PJM can be seen in Figure 13. The figure shows that the 25- and 35-minute windows tend to include part of the variability in the load trend output. The 55- and 65-minute windows over-smooth the output indicating that parts of the load trend are being filtered out and included in the variability. A window of 45 minutes proved to be the best compromise for isolating the variability from the trend.

Because there was a significant amount of 10-minute load data available, an identical analysis was run using that data. The optimum averaging window was found to be 50 minutes. There were two datasets that contained a full year of 1-minute load data. These were also analyzed and the optimum window was again found to be around 45 minutes. 


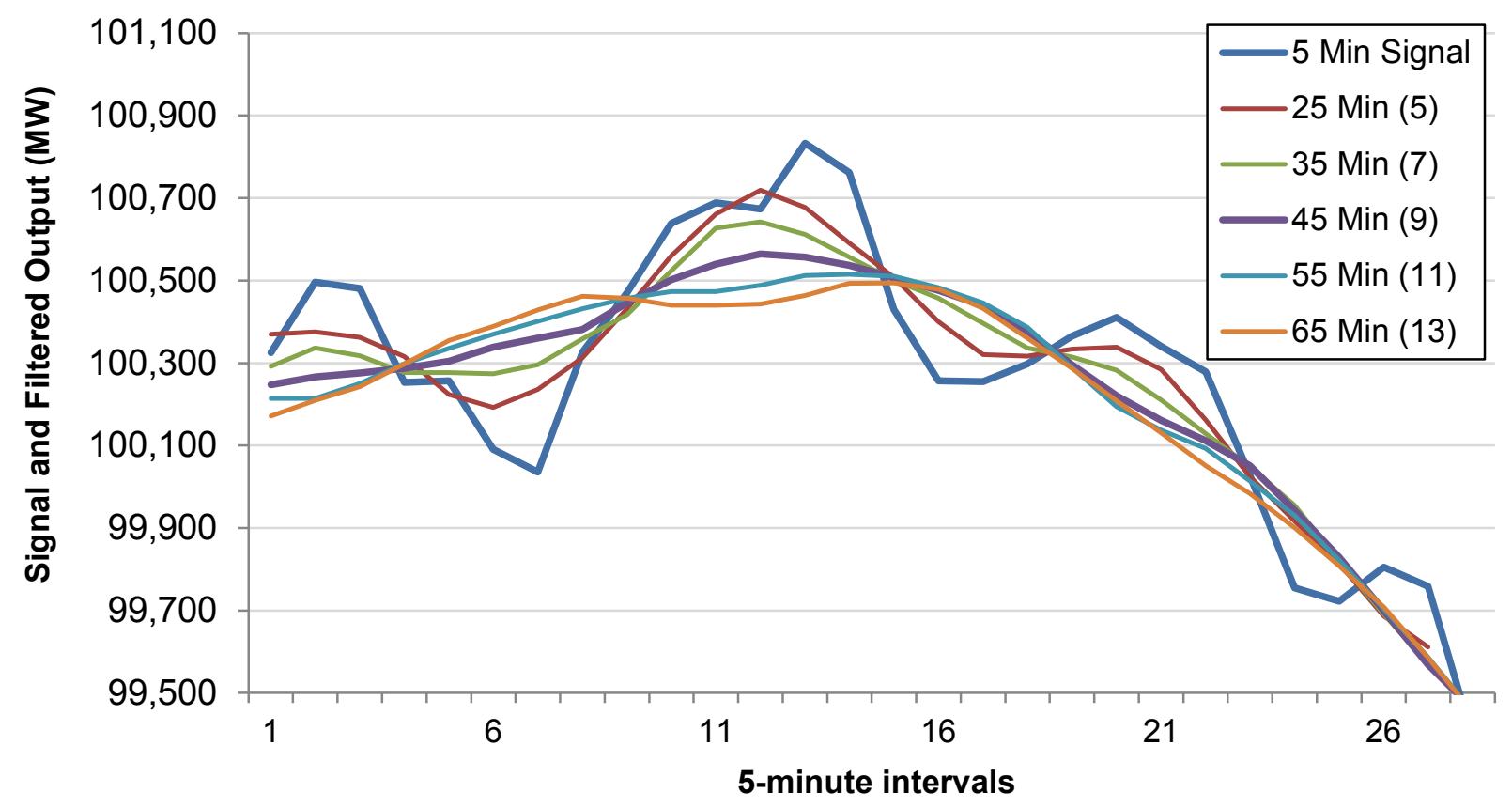

Figure 13. Comparison of various moving average window lengths on filter response for 8:30-10 a.m. for PJM

All of the available datasets were analyzed using the moving window filter with a 45- or 50minute window. One-minute datasets were averaged up to 5 -minute data before the filtering was performed with a 45 -minute window. Datasets at 10 -minute resolution were processed with a 50 minute window. The variability signal was calculated as the differences between the 5- or 10minute resolution data and the trend. The short-term variability was then quantified by the standard deviation of the variability signal.

Figure 14 shows the standard deviation of the short-term variability plotted against the peak load for the region. A trend line is shown for the data shown in blue. The trend shows a clear linear relationship between variability and the size of the region (peak load). 


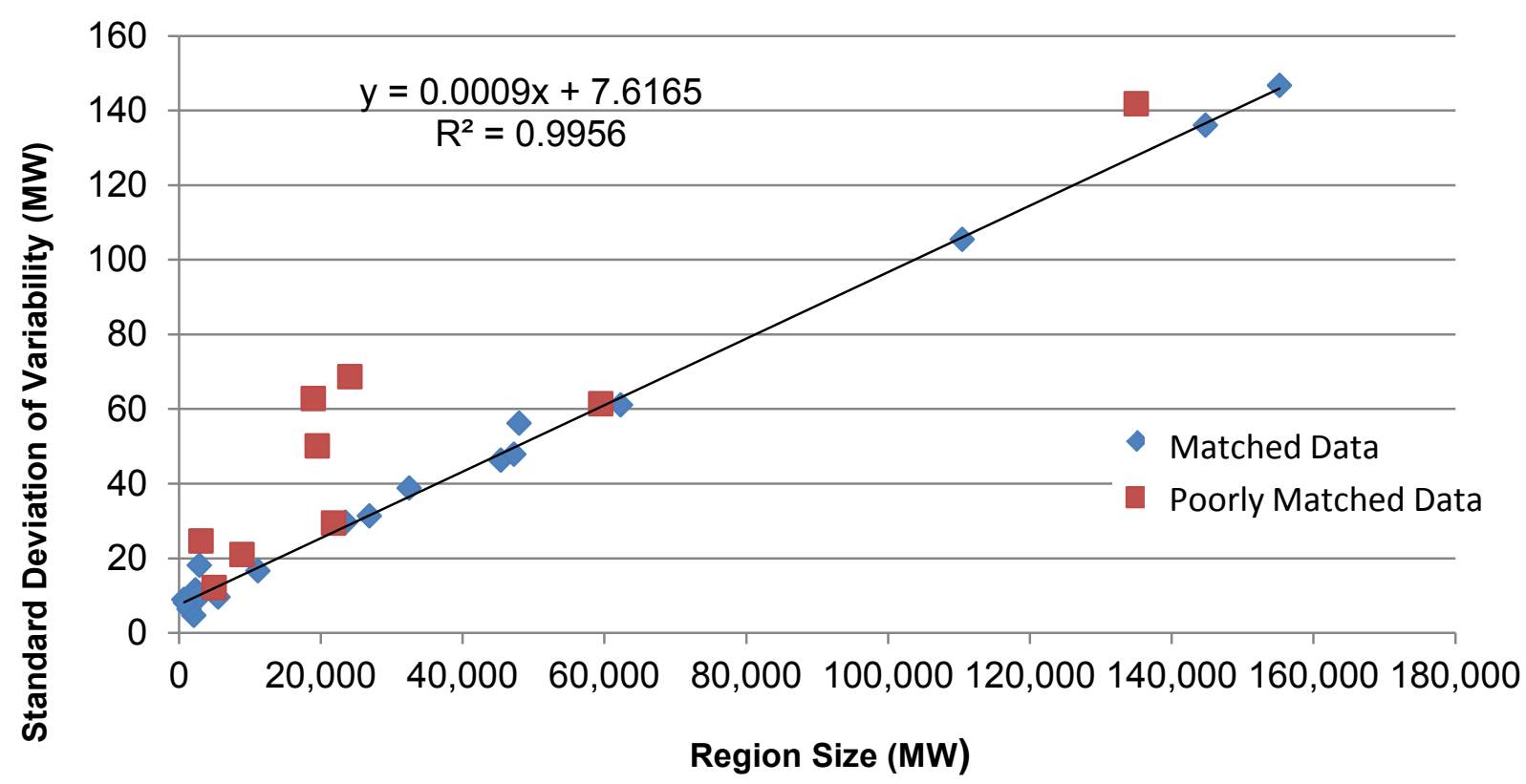

Figure 14. Variability results for high resolution datasets

A smaller subset of the data was analyzed to see if the variability was dependent on season.

Figure 15 shows the results of this analysis. The plots show the variation from season is minimal, and there is not a consistent seasonal pattern seen between the areas.

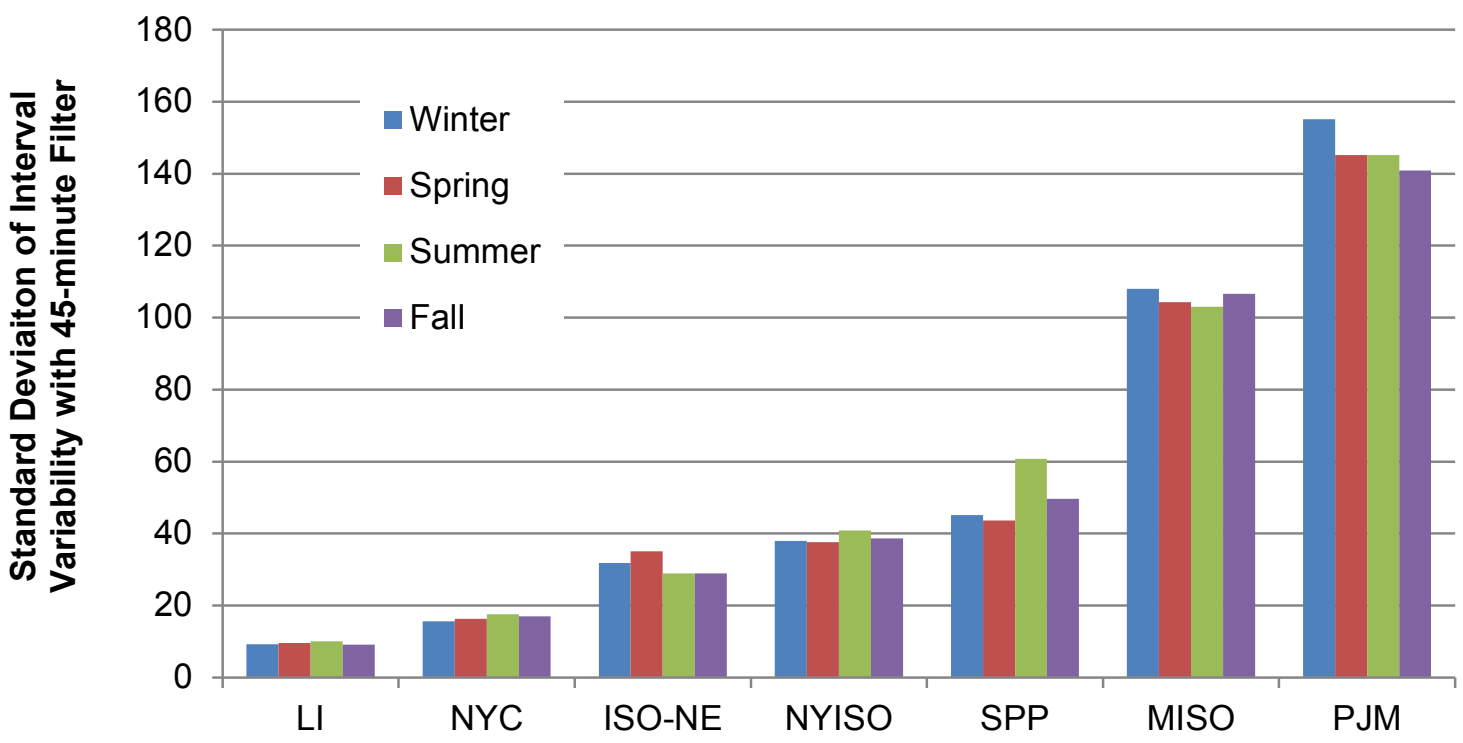

Figure 15. Seasonal variability for Long Island, New York (LI), New York City, New York (NYC), ISO-NE, NYISO, SPP, MISO, PJM 


\subsection{Study Scenario Setup}

In this section NREL details the processes used to site generation from the ReEDS capacity retirement and expansion simulations to the UC\&ED model.

\subsubsection{Conversion of ReEDS Thermal Fleet Results Into PLEXOS Generators}

The ReEDS capacity retirement and expansion results were used to modify the existing thermal generation fleet in the ERGIS regions. As previously explained, ReEDS determines the total amount of capacity to retire, as well as a total amount of new capacity to build, by ReEDS region (Figure 3) and generator type. ReEDS does not model individual generators but instead total capacity in each ReEDS region. Therefore, a method for selecting individual units from the PLEXOS model for retirement was developed for ERGIS. In each region where ReEDS indicated retirements should occur, the generators of that type in that region were retired starting with the smallest units and increasing until the indicated capacity was reached. This method was based on the assumption that smaller units would typically be more expensive to operate or upgrade with new environmental controls and would therefore be the most likely to retire.

ReEDS provides generation retirements on a capacity basis for each ReEDS region. As such, it does not provide individual units when expansion is needed, so a method was developed for sizing and siting new units. In regions where ReEDS indicated new capacity would be built, new units were placed at buses where retirements had occurred. If insufficient capacity had been retired in that region, additional new units were placed on buses on the high-voltage network. Of the eligible resources, ReEDS determined that only CC and CT capacity would be built. For ERGIS, the ReEDS results were rounded so that all new CC units were $500 \mathrm{MW}$ in size and all new CT units were $100 \mathrm{MW}$ in size. ${ }^{15}$ These units were assumed to have low heat rates in their respective categories but otherwise had the same characteristics as the existing units in their categories. Expansion CC units were assumed to have full load heat rates near 6,800 BTU/kWh and expansion CT units were assumed to have heat rates near 9,400 BTU/kWh. In order to prevent degeneracy in the model caused by identical heat rates across units, a small amount of variability was applied to the heat rates for each expansion unit.

\subsubsection{Siting Wind Plants}

The wind data are representative of aggregated wind plants that are the size and location of potential future wind facilities. When the data were created, an effort was made to match existing and planned wind sites with the Eastern Wind Dataset; however, in some instances the existing capacity and ultimate build-out capacity did not match.

Wind datasets for the PLEXOS model were built based upon the ReEDS allocation of wind generation to each of the ReEDS regions in the EI. For each ReEDS region a list of all wind facilities in the NREL Eastern Wind Dataset was created. The list was ordered such that the existing plants would be selected first and then new facilities would be added in descending capacity factor order. The capacity factor was taken as the average capacity factor over the 3 years of data in the Eastern Wind Dataset. Plants were added until the ReEDS generation target

\footnotetext{
${ }^{15}$ Because ReEDS is a linear program, it does not provide discrete generator units. As such, we must create plausible plants based on the data from ReEDS.
} 
was met. ${ }^{16}$ If there were more existing sites in the region than were required to meet the energy target, all of the existing sites were included in the siting. This occurred in several states, such as Minnesota, where in one case the target was calculated at about $8 \mathrm{TWh}$ and the siting from the existing sites contained about $18 \mathrm{TWh}$. The siting lists were compared, and if necessary, the lists were adjusted so that the higher-penetration scenarios were supersets of the lower-penetration scenarios (i.e., all of the LowVG plants were included in the RTx10 scenario and all of the RTx10 plants were included in the RTx30 and ITx30 scenarios). ${ }^{17}$ Offshore wind was also included to meet wind energy targets in the RTx30 and ITx30 scenarios.

\subsubsection{LowVG Wind Resources}

The LowVG scenario contains the VG resources that were in operation in 2012. Table 9 shows the total wind capacity, available energy, and potential energy penetration for each U.S. EI region in the LowVG, and Appendix C provides the same information for each state.

The wind sites in the Eastern Wind Dataset are representative of aggregated wind plants that are the size and location of potential future wind facilities. When the data were created, an effort was made to match existing and planned wind sites with the dataset; however, in some instances the existing capacity and ultimate build-out capacity did not match. Therefore, the installed capacities contained in Table 9 may be different than was actually installed in 2012.

Table 9. LowVG Wind Resources by Region

\begin{tabular}{|c|c|c|c|}
\hline & Capacity (MW) & Available Energy ${ }^{1}$ (GWh) & Potential Penetration ${ }^{1}(\%)$ \\
\hline FRCC & 0 & 0 & $0 \%$ \\
\hline ISO-NE & 770 & 2,495 & $2 \%$ \\
\hline MISO & 12,166 & 43,324 & $6 \%$ \\
\hline NYISO & 1,259 & 4,408 & $3 \%$ \\
\hline PJM & 3,709 & 12,046 & $1 \%$ \\
\hline SERC & 20 & 71 & $0 \%$ \\
\hline SPP & 5,854 & 22,216 & $9 \%$ \\
\hline Total & 23,778 & 84,559 & $3 \%$ \\
\hline
\end{tabular}

${ }^{1}$ This table shows available energy and potential penetration, which are inputs to the PLEXOS model rather than outputs. Actual wind generation and penetration could be lower due to curtailment of available energy.

\subsubsection{RTX10 Wind Resources}

All of the plants in the LowVG scenario were included in the RTx10 scenario, and then additional plants were selected to meet the wind energy requirements as given by the ReEDS model. Table 10 shows the total wind capacity, available energy, and potential energy

\footnotetext{
${ }^{16}$ The ReEDS dataset and Eastern Wind Dataset have different average capacity factors for wind, meaning that 1 MW of wind in the ReEDS model represents a different amount of generation than $1 \mathrm{MW}$ of wind in the Eastern Wind Dataset. Because the ReEDS constraints were formulated in terms of energy penetration, the Eastern Wind Dataset sites were selected to meet the ReEDS energy generation levels rather than the ReEDS capacity amounts.

${ }^{17}$ This method of siting VG generation was developed solely for modeling purposes and does not reflect a number of variables beyond capacity factors that are taken into account when building generation plants.
} 
penetration for each U.S. EI region in the RTx10, and Appendix C provides the same information for each state.

In a few cases there were not sufficient resources available in the NREL Eastern Wind Dataset within a state to satisfy the state's RPS requirement. ${ }^{18}$ Connecticut fell short by more than 2,700 GWh. Additional wind resources were developed in New York and exported to Connecticut to make up this difference. A similar situation happened in Maryland where a target of approximately 10,000 GWh fell short by over 7,300 GWh. Resources from both Virginia and West Virginia were selected to make up this difference. In some cases offshore resources were chosen to meet the RPS target. This was true for Connecticut, Massachusetts, Michigan, North Carolina, New Jersey, and Wisconsin.

Table 10. RTx10 Wind Resources by Region

\begin{tabular}{|l|r|r|r|}
\hline & Capacity (MW) & Available Energy ${ }^{1}(\mathbf{G W h})$ & Potential Penetration $\mathbf{1}$ (\%) \\
\hline FRCC & 0 & 0 & $0 \%$ \\
\hline ISO-NE & 4,024 & 14,406 & $11 \%$ \\
\hline MISO & 40,035 & 142,632 & $18 \%$ \\
\hline NYISO & 9,799 & 35,075 & $21 \%$ \\
\hline PJM & 22,059 & 72,757 & $8 \%$ \\
\hline SERC & 860 & 2,500 & $0 \%$ \\
\hline SPP & 27,521 & 104,154 & $43 \%$ \\
\hline Total & 104,299 & 371,524 & $\mathbf{1 1 \%}$ \\
\hline
\end{tabular}

${ }^{1}$ This table shows available energy and potential penetration, which are inputs to the PLEXOS model rather than outputs. Actual wind generation and penetration could be lower due to curtailment of available energy.

\subsubsection{RTx30 Wind Resources}

All of the plants in the RTx10 scenario were included in the RTx30 scenario, and then additional plants were selected to meet the requirements as given by the ReEDS model. Table 11 shows the total wind capacity, available energy, and potential energy penetration for each U.S. EI region in the RTx30, and Appendix C provides the same information for each state. Offshore wind contributed to the total wind energy for some regions, ranging from $10 \%$ of all wind generation in MISO (Great Lakes) and 80\% of all SERC wind generation (primarily from wind located off the Carolinas).

\footnotetext{
${ }^{18}$ The wind resource is a reflection of the meso-scale weather modeling at $80 \mathrm{~m}$ hub height and does not necessarily represent an upper bound on the actual available wind resource for that area given enabling technologies.
} 
Table 11. RTx30 Wind Resources by Region

\begin{tabular}{|c|c|c|c|}
\hline & Capacity (MW) & Available Energy $^{1}$ (GWh) & Potential Penetration ${ }^{1}(\%)$ \\
\hline FRCC & 0 & 0 & $0 \%$ \\
\hline ISO-NE & 10,053 & 34,627 & $25 \%$ \\
\hline MISO & 55,120 & 199,255 & $26 \%$ \\
\hline NYISO & 10,355 & 36,925 & $22 \%$ \\
\hline PJM & 60,443 & 199,407 & $22 \%$ \\
\hline SERC & 4,223 & 12,677 & $2 \%$ \\
\hline SPP & 42,997 & 164,866 & $68 \%$ \\
\hline Total & 183,191 & 647,758 & $20 \%$ \\
\hline
\end{tabular}

${ }^{1}$ This table shows available energy and potential penetration, which are inputs to the PLEXOS model rather than outputs. Actual wind generation and penetration could be lower due to curtailment of available energy.

\subsubsection{ITx30 Wind Resources}

All of the plants in the RTx 10 scenario were included in the ITx30 scenario, and then additional plants were selected to meet the requirements as given by the ReEDS model. Table 12 shows the total wind capacity, available energy, and potential energy penetration for each U.S. EI region in the ITx30, and Appendix C provides the same information for each state. Offshore wind contributed to the total wind energy for some regions, ranging from $4 \%$ of all wind generation in MISO and $54 \%$ of all SERC wind generation.

Table 12. ITx30 Wind Resources by Region

\begin{tabular}{|c|c|c|c|}
\hline & Capacity (MW) & Available Energy ${ }^{1}$ (GWh) & Potential Penetration ${ }^{1}(\%)$ \\
\hline FRCC & 0 & 0 & $0 \%$ \\
\hline ISO-NE & 8,900 & 30,421 & $22 \%$ \\
\hline MISO & 84,844 & 304,517 & $39 \%$ \\
\hline NYISO & 11,622 & 41,306 & $25 \%$ \\
\hline PJM & 51,848 & 171,403 & $19 \%$ \\
\hline SERC & 4,133 & 12,319 & $2 \%$ \\
\hline SPP & 60,860 & 234,475 & $97 \%$ \\
\hline Total & 222,206 & 794,441 & $25 \%$ \\
\hline
\end{tabular}

Figure 16 shows the wind capacity distribution for each state in the four ERGIS scenarios. 


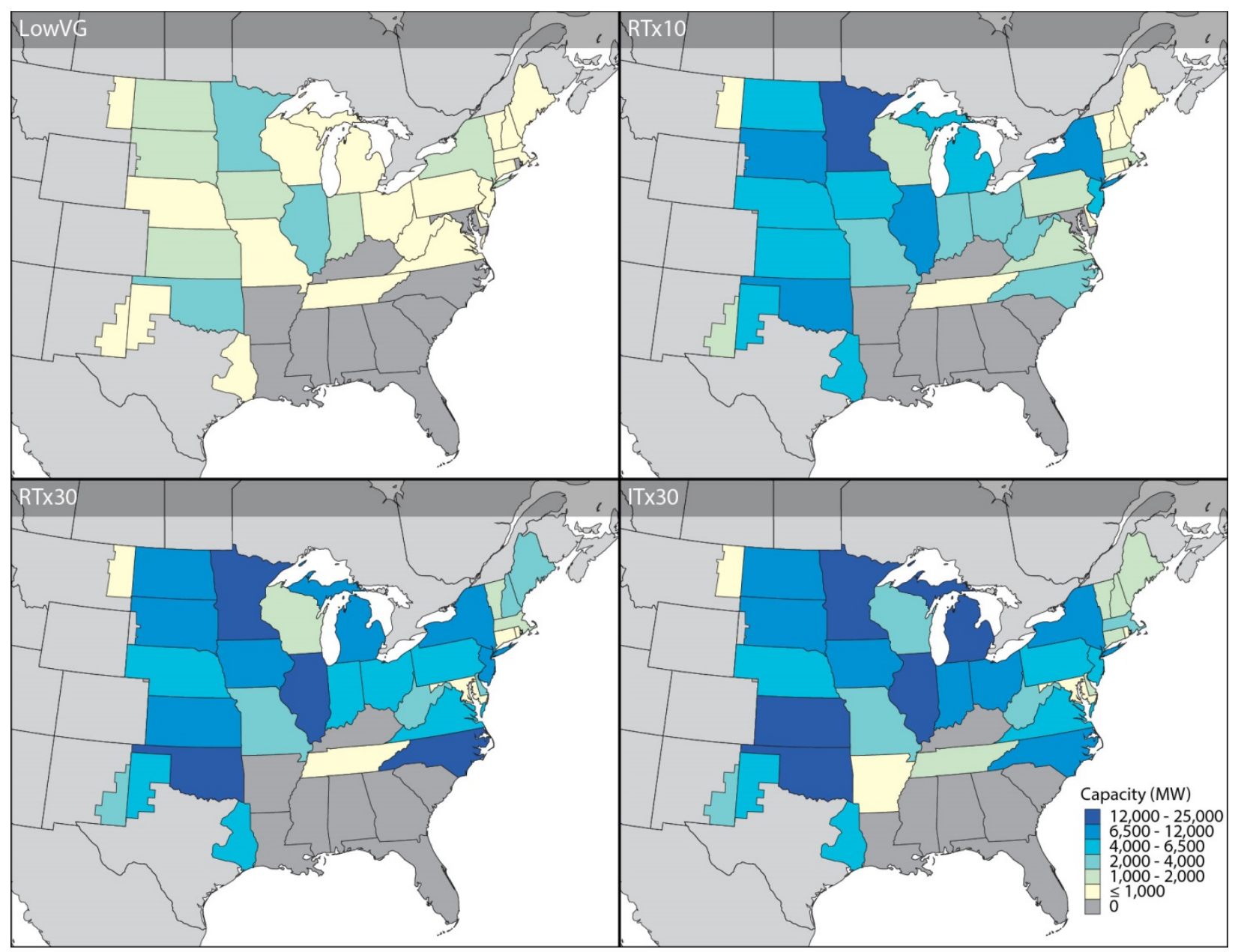

Figure 16. Installed wind capacity by state for each of the ERGIS scenarios: LowVG (first), RTx10 (second), RTx30 (third), ITx30 (fourth)

\subsubsection{Siting PV Plants}

PV resources were sited using one technique for distributed resources (rooftop PV) and another for utility-scale PV facilities. Except as noted below, $60 \%$ of the PV capacity was utility scale $\mathrm{PV}$ and $40 \%$ was distributed.

Distributed PV sites were sited based on the ReEDS results. We started with the required energy for each ReEDS region and then allocated that energy by county in proportion to the county population. Not all states initially had distributed PV allocations based on the ReEDS results; this is because ReEDS constraints were applied at the macro region (RTO/ISO, or NERC region, as shown in Figure 3) and EI levels. We applied a minimum distributed PV penetration level for each region. A $2 \%$ penetration of distributed $\mathrm{PV}$ was assumed for every state in ISO-NE, MISO, NYISO, PJM, and SPP. In SERC, 6\% penetration was assumed for each state. In FRCC, a single state region, we assumed a penetration level of $10 \%$. Where a state did not get enough allocation to fulfill this distributed requirement, the appropriate minimum was applied and that energy was shifted from other states in the macro area.

Several constraints were applied to keep the allocations at reasonable levels and control the number of distributed facilities that would be required. PV capacity requirements were estimated 
by calculating average capacity factors for each region from the ReEDS PV results. Next, the maximum allowable capacity in an area was limited to $1 \mathrm{~kW} /$ person except in Florida where it was limited to $1.5 \mathrm{~kW} /$ person. This target represents a maximum for full build-out of residential and commercial rooftop PV and was used as the maximum for the WWSIS-2 study. A county would not be considered for distributed PV if its population density was below 100 persons/square mile. This limit was used to control the number of counties that would have distributed PV sited by eliminating areas that would have small installed capacity.

Once the total energy for the county was determined, an appropriate number of locations were selected within that county to represent a reasonable energy density. No attempt was made to allocate to population densities below the county level (e.g., by spreading across counties by population), and grids were selected by proximity to the county centroid.

Utility-scale PV plants were sited based on global horizontal irradiance from the NREL global horizontal irradiance resource maps. The energy requirements for each ReEDS region were determined by subtracting any distributed PV from the total required for the region. Estimated capacity requirements were made by calculating average capacity factors for each region and applying those to the energy requirements. For each region the resource and population maps were overlaid. Areas with the best resources were selected first but cities and towns were avoided. Some qualitative effort was made to distribute some of the resources throughout the region even if some lower-quality sites were selected.

\subsubsection{LowVG PV Resources}

The LowVG scenario has very little PV generation. The energy targets for this scenario were developed based on ReEDS region estimates for 2012 production. Based on the ReEDS regions, PV plants were selected from the ERGIS PV dataset to closely match the regional energy targets. Final adjustments were made to exactly match the ReEDS energy through scaling on a regionby-region basis of the ERGIS profiles. Table 13 shows the total PV capacity, available energy, and potential energy penetration for each U.S. EI region in the LowVG, and Appendix C provides the same information for each state.

Table 13. LowVG PV Resources by Region

\begin{tabular}{|l|r|r|r|}
\hline & Capacity (MW) & Available Energy $^{\mathbf{1}}(\mathbf{G W h})$ & Potential Penetration $^{\mathbf{1}}(\%)$ \\
\hline FRCC & 85 & 150 & $0 \%$ \\
\hline ISO-NE & 84 & 107 & $0 \%$ \\
\hline MISO & 6 & 6 & $0 \%$ \\
\hline NYISO & 55 & 76 & $0 \%$ \\
\hline PJM & 483 & 667 & $0 \%$ \\
\hline SERC & 207 & 335 & $0 \%$ \\
\hline SPP & 51 & 109 & $0 \%$ \\
\hline Total & 970 & 1,451 & $0 \%$ \\
\hline
\end{tabular}

${ }^{1}$ This table shows available energy and potential penetration, which are inputs to the PLEXOS model rather than outputs. Actual PV generation and penetration could be lower due to curtailment of available energy. 


\subsubsection{RTx10 PV Resources}

The requirements from ReEDS were matched against the PV sites developed for this study. The requirement was allocated $60 \%$ as utility-scale $\mathrm{PV}$ and $40 \%$ as rooftop (distributed) $\mathrm{PV}$, where possible, based on input from the TRC. Pennsylvania and Ohio had very small allocations to utility-scale PV so the difference was made up by increasing the share of rooftop PV. Table 14 shows the total PV capacity, available energy, and potential energy penetration for each U.S. EI region in the RTx10, and Appendix C provides the same information for each state.

Table 14. RTx10 PV Resources by Region

\begin{tabular}{|l|r|r|r|r|}
\hline & Capacity (MW) & Available Energy $^{\mathbf{1}}(\mathbf{G W h})$ & Potential Penetration $^{\mathbf{1}} \mathbf{( \% )}$ \\
\hline FRCC & 85 & 150 & $0 \%$ \\
\hline ISO-NE & 940 & 1,161 & $1 \%$ \\
\hline MISO & 97 & 142 & $0 \%$ \\
\hline NYISO & 69 & 92 & $0 \%$ \\
\hline PJM & 3,437 & 4,553 & $0 \%$ \\
\hline SERC & 305 & 477 & $0 \%$ \\
\hline SPP & 84 & 151 & $0 \%$ \\
\hline Total & $\mathbf{5 , 0 1 6}$ & $\mathbf{6 , 7 2 6}$ & $\mathbf{0} \%$ \\
\hline
\end{tabular}

${ }^{1}$ This table shows available energy and potential penetration, which are inputs to the PLEXOS model rather than outputs. Actual PV generation and penetration could be lower due to curtailment of available energy.

\subsubsection{RTx30 PV Resources}

Table 15 shows the total PV capacity, available energy, and potential energy penetration for each U.S. EI region in the RTx30, and Appendix C provides the same information for each state.

Table 15. RTx30 PV Resources by Region

\begin{tabular}{|l|r|r|r|}
\hline & Capacity (MW) & Available Energy ${ }^{\mathbf{1}}$ (GWh) & Potential Penetration $\mathbf{1}^{\mathbf{1}}$ ) \\
\hline FRCC & 53,234 & 82,317 & $32 \%$ \\
\hline ISO-NE & 5,657 & 6,888 & $5 \%$ \\
\hline MISO & 34,700 & 46,463 & $6 \%$ \\
\hline NYISO & 6,798 & 8,281 & $5 \%$ \\
\hline PJM & 33,478 & 43,682 & $5 \%$ \\
\hline SERC & 72,683 & 103,350 & $14 \%$ \\
\hline SPP & 12,138 & 19,533 & $8 \%$ \\
\hline Total & $\mathbf{2 1 8 , 6 8 7}$ & $\mathbf{3 1 0 , 5 1 5}$ & $\mathbf{1 0} \%$ \\
\hline
\end{tabular}

${ }^{1}$ This table shows available energy and potential penetration, which are inputs to the PLEXOS model rather than outputs. Actual PV generation and penetration could be lower due to curtailment of available energy. 


\subsubsection{ITx30 PV Resources}

Table 16 shows the total PV capacity, available energy, and potential energy penetration for each U.S. EI region in the ITx30, and Appendix C provides the same information for each state.

\section{Table 16. ITx30 PV Resources by Region}

\begin{tabular}{|l|r|r|r|}
\hline & Capacity (MW) & Available Energy ${ }^{\mathbf{1}}$ (GWh) & Potential Penetration $^{\mathbf{1}} \mathbf{( \% )}$ \\
\hline FRCC & 36,558 & 58,108 & $23 \%$ \\
\hline ISO-NE & 2,301 & 2,897 & $2 \%$ \\
\hline MISO & 16,393 & 23,093 & $3 \%$ \\
\hline NYISO & 3,302 & 4,196 & $3 \%$ \\
\hline PJM & 15,127 & 20,382 & $2 \%$ \\
\hline SERC & 23,823 & 35,700 & $5 \%$ \\
\hline SPP & 12,304 & 20,356 & $8 \%$ \\
\hline Total & $\mathbf{1 0 9 , 8 0 6}$ & $\mathbf{1 6 4 , 7 3 3}$ & $\mathbf{5} \%$ \\
\hline
\end{tabular}

${ }^{1}$ This table shows available energy and potential penetration, which are inputs to the PLEXOS model rather than outputs. Actual PV generation and penetration could be lower due to curtailment of available energy.

Figure 17 shows the PV capacity for each state in the four ERGIS scenarios. 


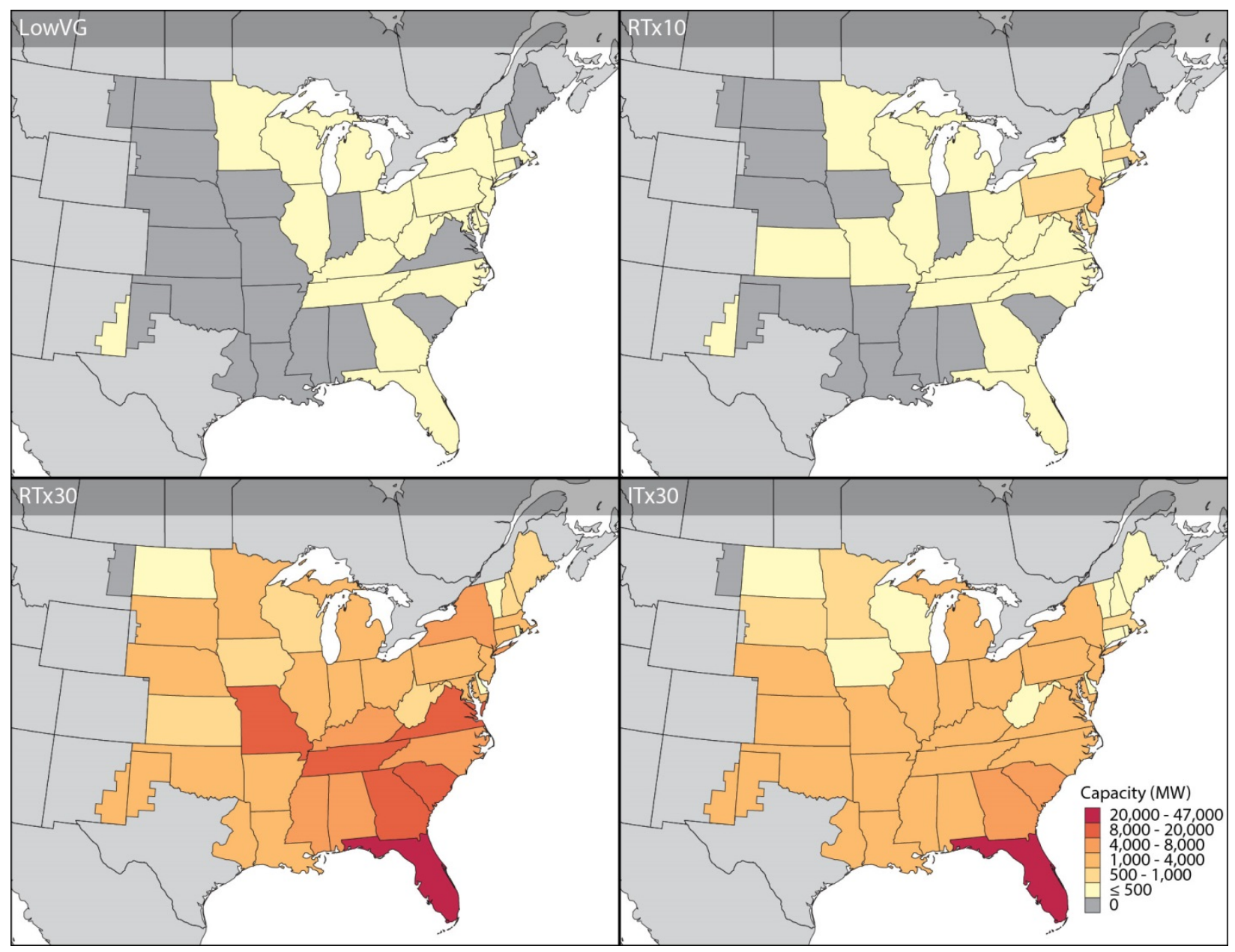

Figure 17. Maps of the installed PV capacity in the four ERGIS scenarios

\subsubsection{ERGIS Regional Details}

Table 17 shows the total installed capacity by generator type of the entire study system for the whole EI (U.S. and Canadian regions) in 2010 and the ERGIS 2026 study scenarios. The Canadian system and the individual U.S. ERGIS regions are described individually below. As noted above, thermal fleet capacity additions and retirements were not optimized for the higher VG scenarios, with the analysis instead using the same thermal fleet for all scenarios to allow for more robust comparisons of differences in VG capacity. Thus, the results in all of the following tables should not be interpreted as indicating that the addition of wind and PV generation does not allow reductions in thermal fleet capacity. 
Table 17. Installed Capacity of the El in 2010 and Each ERGIS Scenario

\begin{tabular}{|c|c|c|c|c|c|}
\hline \multirow{2}{*}{$\begin{array}{l}\text { Generator } \\
\text { Type }\end{array}$} & \multicolumn{5}{|c|}{ Installed Capacity (GW) } \\
\hline & 2010 & LowVG & RTx10 & RTx30 & ITx30 \\
\hline Wind & 7 & 24 & 104 & 183 & 222 \\
\hline PV & 0 & 1 & 5 & 219 & 110 \\
\hline Nuclear & 105 & \multicolumn{4}{|c|}{102} \\
\hline Coal & 298 & \multicolumn{4}{|c|}{230} \\
\hline Gas CC & 165 & \multicolumn{4}{|c|}{173} \\
\hline $\begin{array}{l}\text { CT/Gas } \\
\text { Boiler }\end{array}$ & 166 & \multicolumn{4}{|c|}{148} \\
\hline Hydro & 85 & \multicolumn{4}{|c|}{87} \\
\hline Other & 55 & \multicolumn{4}{|c|}{22} \\
\hline
\end{tabular}

\subsubsection{Canadian Provinces}

ERGIS includes a full representation of each of the Canadian provinces connected to the U.S. EI. Each province is modeled as its own region in the model, with identical modeling treatment to U.S. regions. Table 18 shows the total installed capacity by generator type of the Canadian provinces.

Table 18. Installed Capacity of the Canadian Provinces in 2010 and Each ERGIS Scenario

\begin{tabular}{|c|c|c|c|c|c|}
\hline \multirow{2}{*}{$\begin{array}{l}\text { Generator } \\
\text { Type }\end{array}$} & \multicolumn{5}{|c|}{ Installed Capacity (GW) } \\
\hline & 2010 & LowVG & RTx10 & RTx30 & ITx30 \\
\hline Wind & 0 & 0 & 0 & 0 & 0 \\
\hline PV & 0 & 0 & 0 & 0 & 0 \\
\hline Nuclear & 15 & \multicolumn{4}{|c|}{12} \\
\hline Coal & 13 & \multicolumn{4}{|c|}{7} \\
\hline Gas CC & 7 & \multicolumn{4}{|c|}{5} \\
\hline $\begin{array}{l}\text { CT/Gas } \\
\text { Boiler }\end{array}$ & 7 & \multicolumn{4}{|c|}{3} \\
\hline Hydro & 58 & \multicolumn{4}{|c|}{59} \\
\hline Other & 1 & \multicolumn{4}{|c|}{0} \\
\hline
\end{tabular}

\subsubsection{Florida Reliability Coordinating Council}

FRCC is a NERC regional entity. All generators and transmission elements in this region are assumed to be coordinated within the FRCC footprint. The region includes a variety of municipal and cooperative power systems as well as several independent power producers and investorowned utilities. Table 19 shows the total installed capacity by generator type of the FRCC region used in each ERGIS Scenario. 
Table 19. Installed Capacity of FRCC in 2010 and Each ERGIS Scenario

\begin{tabular}{|c|c|c|c|c|c|}
\hline \multirow{2}{*}{$\begin{array}{l}\text { Generator } \\
\text { Type }\end{array}$} & \multicolumn{5}{|c|}{ Installed Capacity (GW) } \\
\hline & 2010 & LowVG & RTx10 & RTx30 & ITx30 \\
\hline Wind & 0 & 0 & 0 & 0 & 0 \\
\hline PV & 0 & 0 & 0 & 53 & 37 \\
\hline Nuclear & 3 & \multicolumn{4}{|c|}{3} \\
\hline Coal & 11 & \multicolumn{4}{|c|}{10} \\
\hline Gas CC & 26 & \multicolumn{4}{|c|}{42} \\
\hline $\begin{array}{l}\text { CT/Gas } \\
\text { Boiler }\end{array}$ & 17 & \multicolumn{4}{|c|}{10} \\
\hline Hydro & 0 & \multicolumn{4}{|c|}{0} \\
\hline Other & 4 & \multicolumn{4}{|c|}{0} \\
\hline
\end{tabular}

\subsubsection{SERC Reliability Corporation}

SERC is a NERC regional entity for much of the central and southeastern United States. In this study, NREL combines the generation and transmission assets of Georgia, the Florida panhandle, Alabama, South Carolina, North Carolina, Tennessee, and parts of Kentucky and Mississippi into one region. The most notable difference in the traditional SERC boundaries and the ERGIS regions is with respect to SERC regional members that are part of an RTO/ISO. Bulk power facilities associated with the SERC reliability region, which are members of an RTO/ISO, are modeled as members of the RTO/ISO region, and not the NERC reliability region. For example, the Entergy Corporation is located in the SERC reliability region, but is a participant in the MISO market. As such, Entergy is included in the MISO region. Table 20 shows the total installed capacity by generator type in the SERC region.

Table 20. Installed Capacity of SERC in 2010 and Each ERGIS Scenario

\begin{tabular}{|c|c|c|c|c|c|}
\hline \multirow{2}{*}{$\begin{array}{l}\text { Generator } \\
\text { Type }\end{array}$} & \multicolumn{5}{|c|}{ Installed Capacity (GW) } \\
\hline & 2010 & LowVG & RTx10 & RTx30 & ITx30 \\
\hline Wind & 0 & 0 & 1 & 4 & 4 \\
\hline PV & 0 & 0 & 0 & 73 & 24 \\
\hline Nuclear & 25 & \multicolumn{4}{|c|}{25} \\
\hline Coal & 64 & \multicolumn{4}{|c|}{49} \\
\hline Gas CC & 29 & \multicolumn{4}{|c|}{23} \\
\hline $\begin{array}{l}\text { CT/Gas } \\
\text { Boiler }\end{array}$ & 28 & \multicolumn{4}{|c|}{22} \\
\hline Hydro & 11 & \multicolumn{4}{|c|}{11} \\
\hline Other & 10 & \multicolumn{4}{|c|}{6} \\
\hline
\end{tabular}




\subsubsection{Midcontinent Independent System Operator}

MISO is an RTO/ISO market region and the largest geographic region in ERGIS. While the multi-year ERGIS study was underway, Entergy joined MISO, enlarging its footprint to extend from the Gulf of Mexico in Mississippi to the northern-most states of the contiguous United States. The modeled footprint of MISO generally follows the market footprint of MISO, with a few noteworthy exceptions. ${ }^{19}$ First, the non-RTO/ISO service territories of Missouri that are neither part of MISO nor the SPP were included as part of MISO to simplify modeling. Another deviation from current borders is the inclusion of the Western Area Power Administration (Western), Basin Electric Power Cooperative, and Heartland Consumers Power District in the MISO ERGIS region. In late 2014 these regions received approval to join SPP from the Federal Energy Regulatory Commission (FERC). Because the ERGIS regions were formed in 2013, NREL was unable to update the market footprint for the study. Table 21 shows the total installed capacity by generator type of the MISO region.

Table 21. Installed Capacity of MISO in 2010 and Each ERGIS Scenario

\begin{tabular}{|c|c|c|c|c|c|}
\hline \multirow{2}{*}{$\begin{array}{l}\text { Generator } \\
\text { Type }\end{array}$} & \multicolumn{5}{|c|}{ Installed Capacity (GW) } \\
\hline & 2010 & LowVG & RTx10 & RTx30 & ITx30 \\
\hline Wind & 4 & 12 & 40 & 55 & 85 \\
\hline PV & 0 & 0 & 0 & 35 & 16 \\
\hline Nuclear & 15 & \multicolumn{4}{|c|}{15} \\
\hline Coal & 90 & \multicolumn{4}{|c|}{75} \\
\hline Gas CC & 38 & \multicolumn{4}{|c|}{26} \\
\hline $\begin{array}{l}\text { CT/Gas } \\
\text { Boiler }\end{array}$ & 43 & \multicolumn{4}{|c|}{31} \\
\hline Hydro & 5 & \multicolumn{4}{|c|}{5} \\
\hline Other & 8 & \multicolumn{4}{|c|}{4} \\
\hline
\end{tabular}

\subsubsection{Southwest Power Pool}

The SPP ERGIS region extends from the far western edge of the U.S. EI in New Mexico to northern Louisiana and Nebraska. In this study, NREL adopted the SPP RTO/ISO market region boundaries as they existed in 2013. As discussed previously, several organizations that were recently approved to join the SPP are modeled as members of MISO. It is to be expected that this difference in modeling representation and actual system footprints will result in some inherent differences in the regional impact of high penetrations of renewables. Conceivably, because the geographic extent of SPP is larger, in reality, than is modeled in our study, the regional impacts of high penetration of wind and PV in SPP may be mitigated by the geographic diversity of the expanded real market region.

Table 22 shows the total installed capacity by generator type of the SPP region.

\footnotetext{
${ }^{19}$ The benchmarking of the 2010 model in section 4.1.3 was performed with the MISO footprint that excludes Entergy, but was updated for the 2026 model.
} 
Table 22. Installed Capacity of SPP in 2010 and Each ERGIS Scenario

\begin{tabular}{|c|c|c|c|c|c|}
\hline \multirow{2}{*}{$\begin{array}{l}\text { Generator } \\
\text { Type }\end{array}$} & \multicolumn{5}{|c|}{ Installed Capacity (GW) } \\
\hline & 2010 & LowVG & RTx10 & RT $\times 30$ & ITx30 \\
\hline Wind & 2 & 6 & 28 & 43 & 61 \\
\hline PV & 0 & 0 & 0 & 12 & 12 \\
\hline Nuclear & 3 & \multicolumn{4}{|c|}{3} \\
\hline Coal & 27 & \multicolumn{4}{|c|}{22} \\
\hline Gas CC & 14 & \multicolumn{4}{|c|}{9} \\
\hline $\begin{array}{l}\text { CT/Gas } \\
\text { Boiler }\end{array}$ & 20 & \multicolumn{4}{|c|}{11} \\
\hline Hydro & 2 & \multicolumn{4}{|c|}{2} \\
\hline Other & 2 & \multicolumn{4}{|c|}{0} \\
\hline
\end{tabular}

\subsubsection{PJM Interconnection}

PJM is the largest power pool in North America. Though geographically smaller than many other ERGIS regions, PJM has the largest load in the study. The ERGIS region for PJM largely mimics the actual market region in 2013. Table 23 shows the total installed capacity by generator type of PJM.

Table 23. Installed Capacity of PJM in 2010 and Each ERGIS Scenario

\begin{tabular}{|c|c|c|c|c|c|}
\hline \multirow{2}{*}{$\begin{array}{l}\text { Generator } \\
\text { Type }\end{array}$} & \multicolumn{5}{|c|}{ Installed Capacity (GW) } \\
\hline & 2010 & LowVG & RTx10 & RTx30 & ITx30 \\
\hline Wind & 0 & 4 & 22 & 50 & 52 \\
\hline PV & 0 & 0 & 3 & 33 & 15 \\
\hline Nuclear & 34 & \multicolumn{4}{|c|}{34} \\
\hline Coal & 87 & \multicolumn{4}{|c|}{62} \\
\hline Gas CC & 28 & \multicolumn{4}{|c|}{50} \\
\hline $\begin{array}{l}\text { CT/Gas } \\
\text { Boiler }\end{array}$ & 36 & \multicolumn{4}{|c|}{52} \\
\hline Hydro & 3 & \multicolumn{4}{|c|}{3} \\
\hline Other & 17 & \multicolumn{4}{|c|}{7} \\
\hline
\end{tabular}

\subsubsection{New York Independent System Operator}

NYISO is a single-state independent system operator and is modeled as such in ERGIS. Table 24 shows the total installed capacity by generator type of NYISO. 
Table 24. Installed Capacity of NYISO in 2010 and Each ERGIS Scenario

\begin{tabular}{|c|c|c|c|c|c|}
\hline \multirow{2}{*}{$\begin{array}{l}\text { Generator } \\
\text { Type }\end{array}$} & \multicolumn{5}{|c|}{ Installed Capacity (GW) } \\
\hline & 2010 & LowVG & RTx10 & RTx30 & ITx30 \\
\hline Wind & 0 & 1 & 10 & 10 & 12 \\
\hline PV & 0 & 0 & 0 & 7 & 3 \\
\hline Nuclear & 5 & \multicolumn{4}{|c|}{5} \\
\hline Coal & 3 & \multicolumn{4}{|c|}{2} \\
\hline Gas CC & 9 & \multicolumn{4}{|c|}{8} \\
\hline $\begin{array}{l}\text { CT/Gas } \\
\text { Boiler }\end{array}$ & 13 & \multicolumn{4}{|c|}{12} \\
\hline Hydro & 5 & \multicolumn{4}{|c|}{5} \\
\hline Other & 5 & \multicolumn{4}{|c|}{1} \\
\hline
\end{tabular}

\subsubsection{New England Independent System Operator}

ISO-NE is comprised of all resources and associated transmission elements in the New England states of Connecticut, Maine, Massachusetts, New Hampshire, Rhode Island, and Vermont. The modeled ISO-NE region is exactly the same as the market region. Table 25 shows the total installed capacity by generator type of ISO-NE.

Table 25. Installed Capacity of ISO-NE in 2010 and Each ERGIS Scenario

\begin{tabular}{|c|c|c|c|c|c|}
\hline \multirow{2}{*}{$\begin{array}{l}\text { Generator } \\
\text { Type }\end{array}$} & \multicolumn{5}{|c|}{ Installed Capacity (GW) } \\
\hline & 2010 & LowVG & RTx10 & RTx30 & ITx30 \\
\hline Wind & 0 & 1 & 4 & 10 & 9 \\
\hline PV & 0 & 0 & 1 & 6 & 2 \\
\hline Nuclear & 5 & \multicolumn{4}{|c|}{5} \\
\hline Coal & 4 & \multicolumn{4}{|c|}{2} \\
\hline Gas CC & 14 & \multicolumn{4}{|c|}{10} \\
\hline $\begin{array}{l}\text { CT/Gas } \\
\text { Boiler }\end{array}$ & 3 & \multicolumn{4}{|c|}{6} \\
\hline Hydro & 2 & \multicolumn{4}{|c|}{2} \\
\hline Other & 8 & \multicolumn{4}{|c|}{3} \\
\hline
\end{tabular}

\subsubsection{EIPC Transmission Expansions}

Because ERGIS is not a transmission planning study, we adopted the transmission expansions created by the EIPC Phase II study and applied them to the ERGIS scenarios. The EIPC Phase II study generated three transmission expansions targeted at three different future scenarios (EIPC 2012). ERGIS did not adopt the policy assumptions or generation portfolios that led to the development of any of the EIPC's transmission expansion scenarios; ERGIS merely adopted the transmission build-out scenarios. 
EIPC's Scenario 3 assumed business-as-usual conditions, with comparatively small additions of transmission capacity. This scenario included expansion of generation capacity to meet future load, with minor VG expansion, most similar to the ERGIS LowVG scenario. Differences include the projected year of 2030 as well as conventional generation expansions that are not optimized with the transmission. Despite these differences, the TRC determined that this transmission expansion was a reasonable approximation of what might work well in a future power system with small VG growth. Figure 18 shows EIPC's Scenario 3 transmission expansion.

EIPC's Scenario 2 assumed that a national RPS of 30\% of the nation's electricity from VG was implemented on a regional basis. This transmission expansion was adopted for the ERGIS RTx10 and RTx30 scenarios and includes considerable intra-regional transmission builds. The TRC determined that this transmission expansion was most in line with a VG deployment scenario largely driven by state or regional action. Figure 19 shows EIPC's Scenario 2 transmission expansion.

EIPC's Scenario 1 assumed that a nationally implemented carbon constraint was put in place. This resulted in large deployments of energy efficiency and demand response, as well as a large expansion of renewable generation technologies. ${ }^{20}$ This scenario also included a large transmission network expansion to support exchanging large amounts of power between regions. This transmission expansion was the largest of the three EIPC scenarios and features several HVDC lines. This transmission expansion was adopted for the ERGIS ITx30 scenario. A map of EIPC's Scenario 1 transmission expansion is shown in Figure 20.

${ }^{20}$ The ERGIS ITx30 scenario, however, had an identical load as the other ERGIS scenarios. 


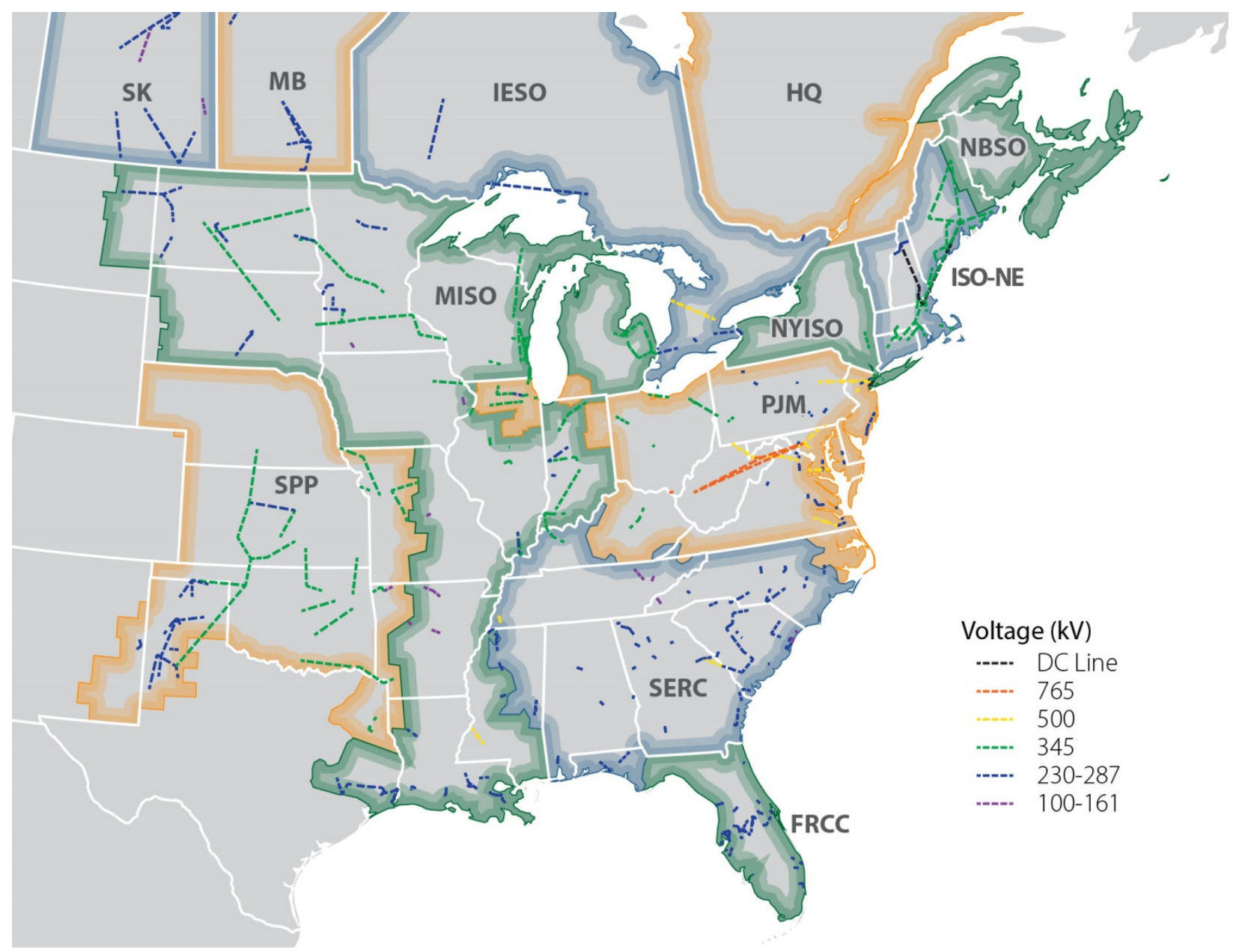

Figure 18. Map showing the EIPC Phase II Scenario 3 transmission expansion overlaid on the ERGIS regions. This expansion was used in the LowVG. 


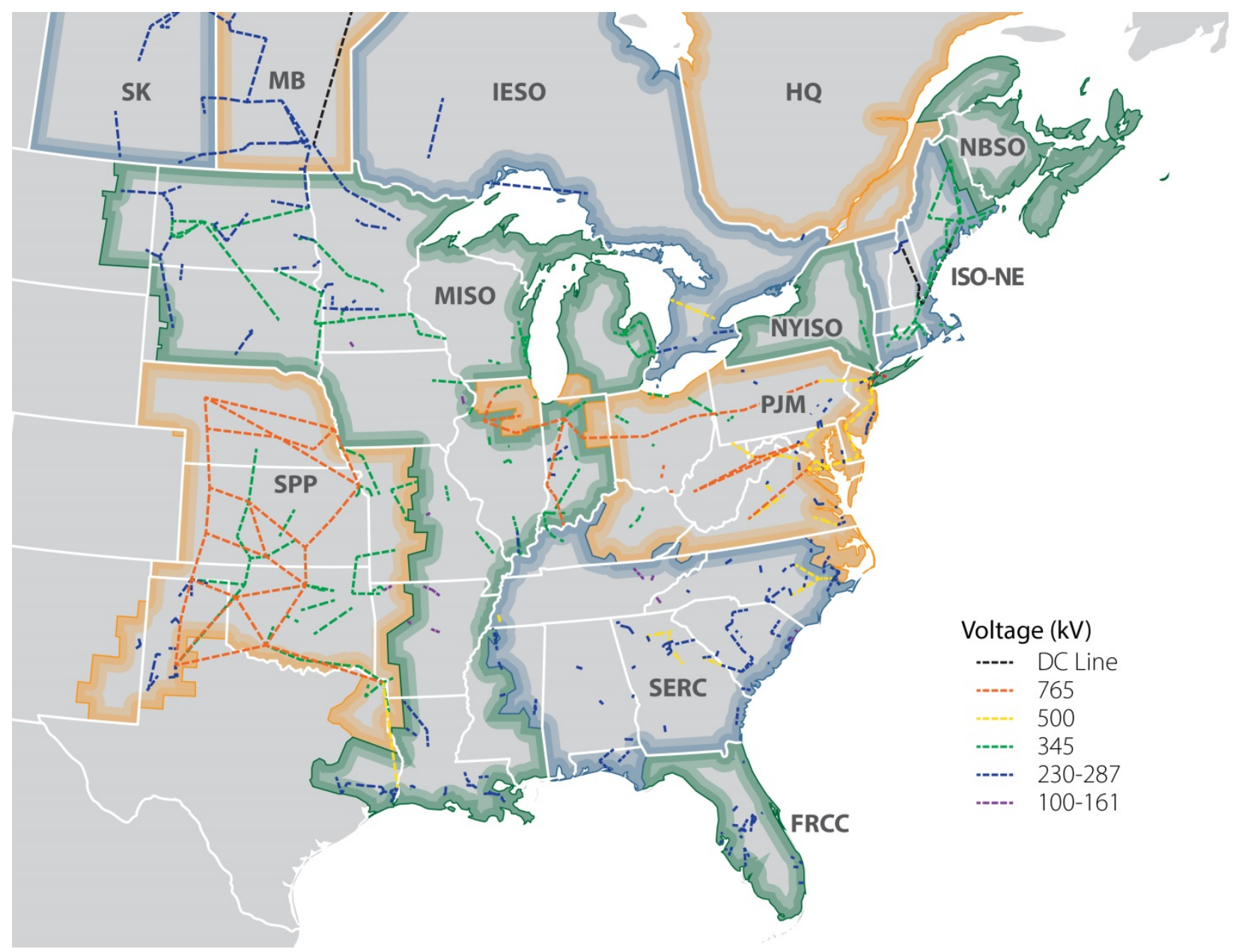

Figure 19. Map showing the EIPC Phase II Scenario 2 transmission expansion overlaid on the ERGIS regions. This expansion was used in RTx10 and RTx30. 


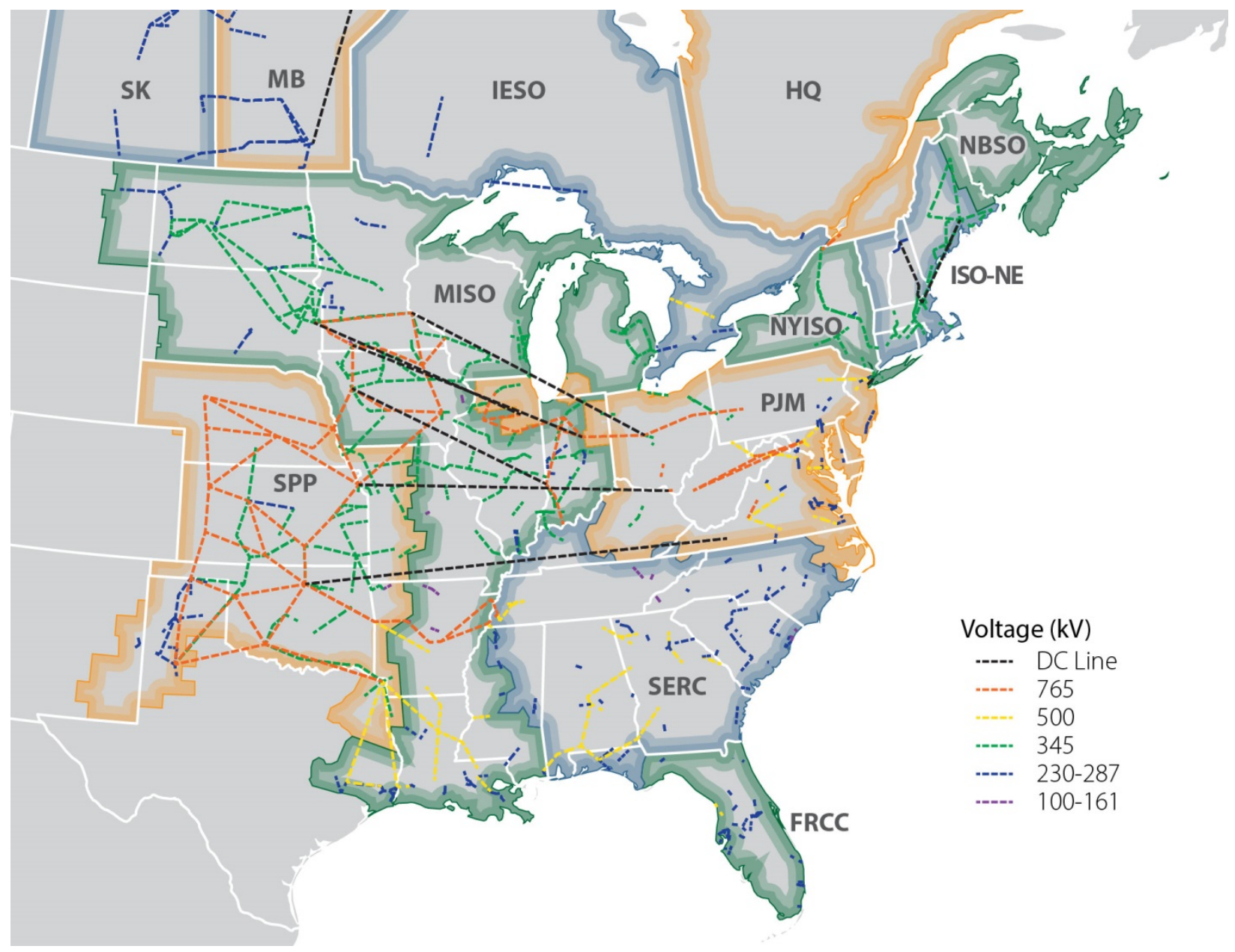

Figure 20. Map showing the EIPC Phase II Scenario 1 transmission expansion overlaid on the ERGIS regions. This expansion was used in ITx30. 


\section{Analysis of Wind, PV, and Load Data}

An analysis was performed to understand the characteristics of the aggregate VG data and how it interacts with the load. This analysis examined the nature and variability of wind and PV generation and net load. Note that this analysis used input data to the UC\&ED model rather than output data, and any VG curtailment by the model would change these results somewhat. This analysis also aggregates all load and VG data to the interconnection level and therefore ignores transmission constraints.

\subsection{Annual Analysis}

Table 26 shows a summary of the load and VG statistics for the ERGIS scenarios. The load data are constant across the four scenarios. Each of the scenarios deploys different wind and PV resources, as described in the previous chapter. The ITx30 has a significantly lower total VG generation maximum than the RTx30, indicating that the ITx30 has fewer extremes in output levels than the RTx30. This translates to a significantly higher net load minimum and a somewhat lower net load maximum in the ITx30 relative to the RTx30. In addition, the maximum net load decreased by $39 \mathrm{GW}$ in the RTx10, $109 \mathrm{GW}$ in the RTx30, and $114 \mathrm{GW}$ in the ITx30 relative to the LowVG.

Table 26. General Load and VG Data Statistics

\begin{tabular}{|c|c|c|c|c|c|}
\hline & \multicolumn{4}{|c|}{ Scenario } \\
\hline & & LowVG & RTx10 & RTx30 & IT X30 \\
\hline \multirow{4}{*}{ ర్ర్ర } & Minimum (GW) & 243 & 243 & 243 & 243 \\
\hline & Maximum (GW) & 633 & 633 & 633 & 633 \\
\hline & Total Energy (TWh) & 3,238 & 3,238 & 3,238 & 3,238 \\
\hline & Load Factor (\%) & $58 \%$ & $58 \%$ & $58 \%$ & $58 \%$ \\
\hline \multirow{2}{*}{ ฉ } & Maximum (GW) & 1 & 4 & 150 & 74 \\
\hline & Total Energy (TWh) & 1 & 7 & 311 & 165 \\
\hline \multirow{2}{*}{$\stackrel{\text { 든 }}{3}$} & Maximum (GW) & 20 & 84 & 147 & 174 \\
\hline & Total Energy (TWh) & 85 & 372 & 648 & 794 \\
\hline \multirow{2}{*}{$\stackrel{\sqrt[\pi]{ \pm}}{\stackrel{0}{\circ}}$} & Maximum (GW) & 20 & 86 & 266 & 224 \\
\hline & Total Energy (TWh) & 86 & 378 & 958 & 959 \\
\hline \multirow{4}{*}{ 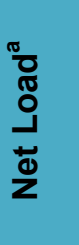 } & Minimum (GW) & 227 & 178 & 70 & 103 \\
\hline & Maximum (GW) & 622 & 583 & 513 & 508 \\
\hline & Total Energy (TWh) & 3,152 & 2,860 & 2,280 & 2,279 \\
\hline & Net Load Factor ${ }^{b}(\%)$ & $58 \%$ & $56 \%$ & $51 \%$ & $51 \%$ \\
\hline \multirow{3}{*}{ 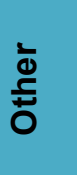 } & Effective Peak Reduction ${ }^{\mathrm{c}}$ (GW) & 11 & 50 & 120 & 125 \\
\hline & Penetration at Peak Load (\%) & $2 \%$ & $8 \%$ & $31 \%$ & $25 \%$ \\
\hline & Average Penetration (\%) & $3 \%$ & $12 \%$ & $30 \%$ & $30 \%$ \\
\hline
\end{tabular}

${ }^{a}$ Net load is defined as actual load minus wind and PV generation.

${ }^{\mathrm{b}}$ Net load factor is average net load divided by peak net load

${ }^{\mathrm{c}}$ Effective peak reduction is peak load minus peak net load. 
Figure 21 shows duration plots of the available energy from wind, PV, total VG, and the net load, for the four study scenarios. The total VG production is produced by summing the wind and PV time series and then sorting for the duration plot.

The LowVG and RTx10 scenarios contain almost no PV so VG duration curves are very similar to the wind-only curves. The LowVG scenario contains minimal VG resources so the impact on the net load curve is also minimal.

The RTx30 scenario has approximately twice the PV of the ITx30 scenario, as can be seen in the PV plot. Note that the two 30\% scenarios have significantly different peak values and the ITx30 scenario provides a slightly flatter duration curve. This means that the VG production varies less over the year in this scenario compared to the RTx30 scenario. The area under the curves is the same because both the RTx30 and ITx30 had 30\% VG target.
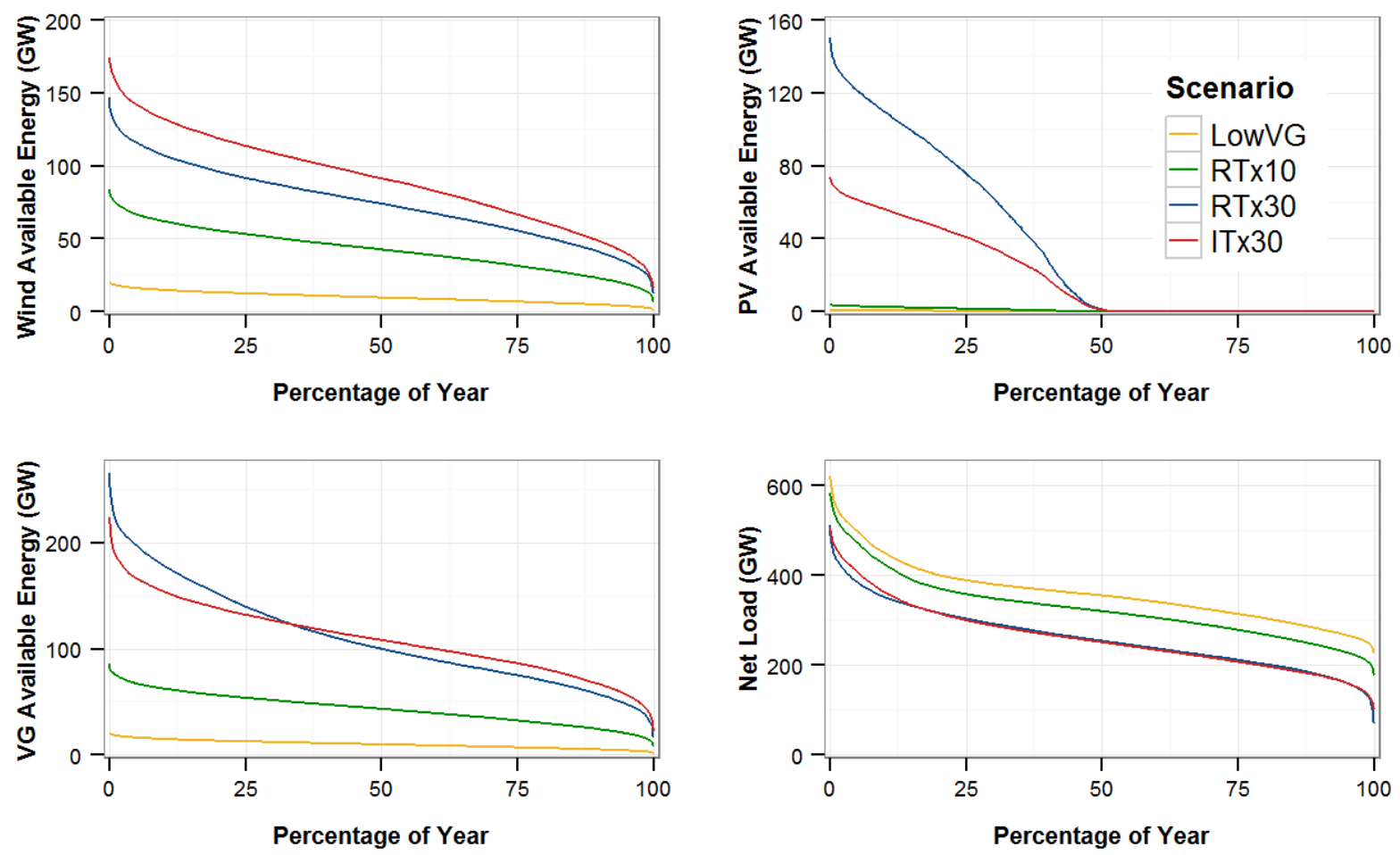

Figure 21. Duration plots for available energy from wind, PV, and total VG, and net load. Note that the $y$-axis scales are different on the each of these graphs.

\subsection{Monthly Available Energy}

Figure 22 shows how wind and PV generation vary on a monthly basis for the four scenarios. Recall that the RTx30 scenario has $20 \%$ wind and 10\% PV energy penetration while the ITx30 scenario has $25 \%$ wind and $5 \% \mathrm{PV}$ penetration. 

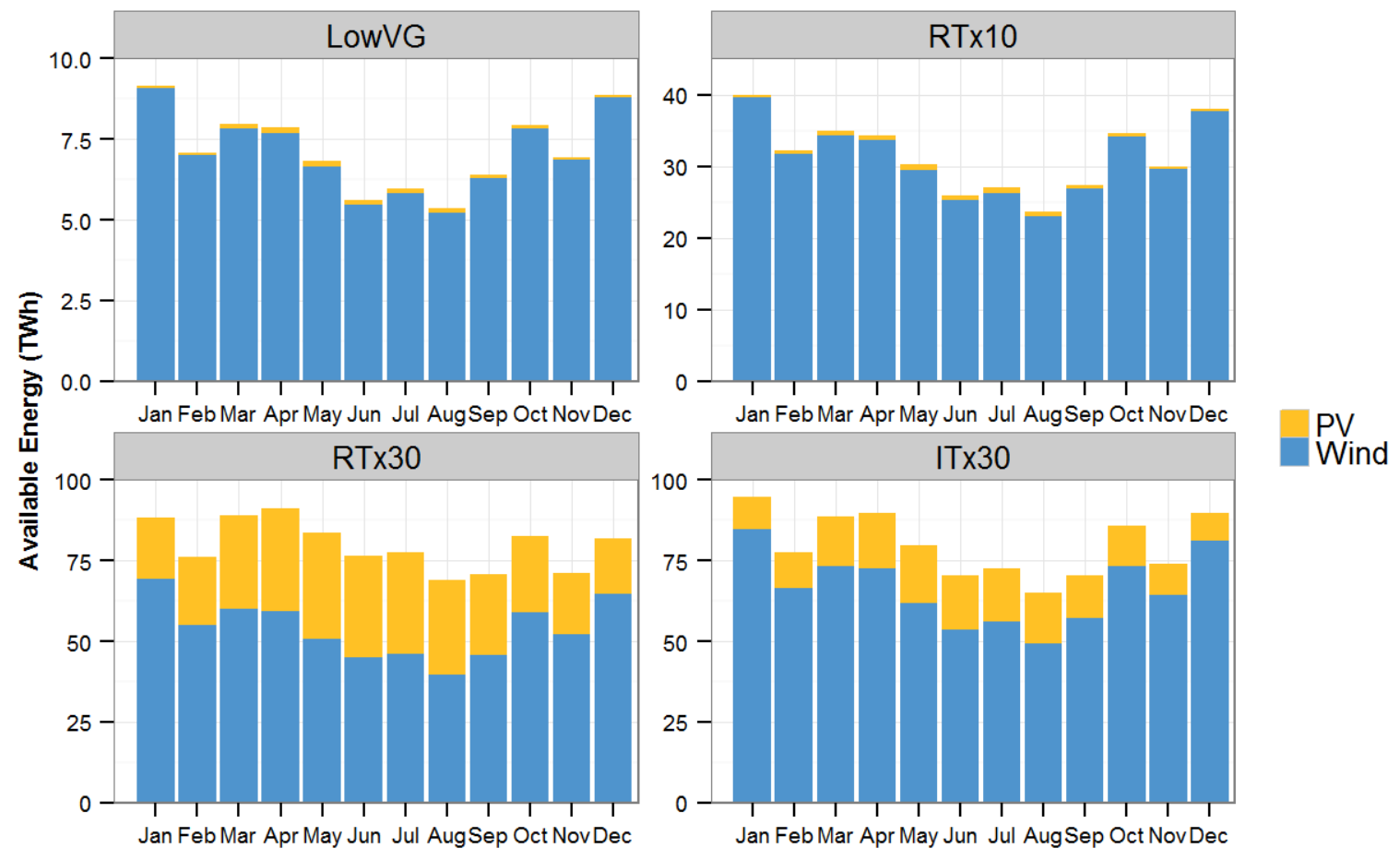

Figure 22. Monthly total VG available energy for the four scenarios. Note that the $y$-axis scales are different on each of these graphs.

Figure 23 shows a different view of the monthly production data by looking at each resource separately for the four scenarios. Wind resources follow a pattern with maximum production in the winter months and minimum production during the summer. PV production peaks in the late spring and is at its minimum in winter. The combination of these characteristics leads to a total VG that is flatter across the months than just wind or PV. VG tends to peak in winter and early spring and drop to its minimum in summer.
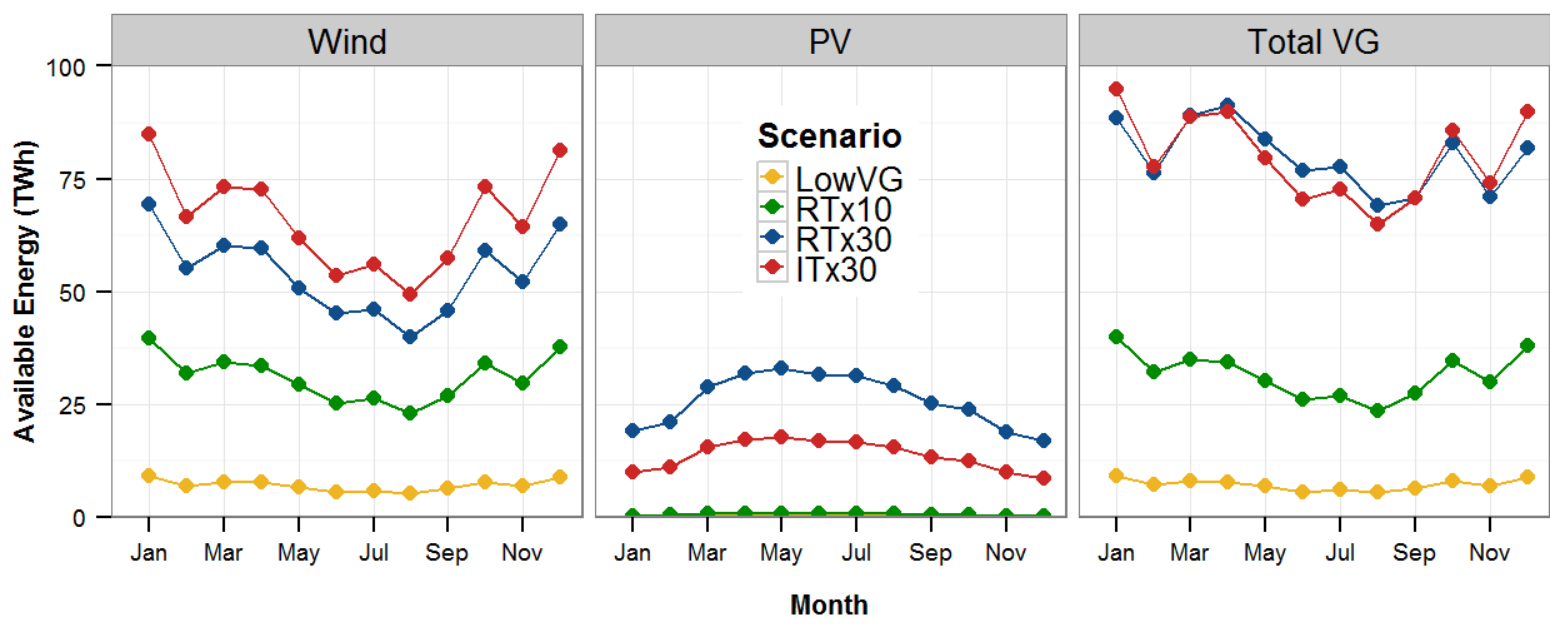

Figure 23. Wind, PV, and total VG available energy by month and scenario. 


\subsection{Forecast Error Analysis}

Accuracy of the unit commitment schedules in the day-ahead depends on the accuracy of the day-ahead forecasts for load, wind, and PV. Load forecasts were not available, so load forecasts were assumed to be perfect, except for the addition of 5-minute variability in the real-time. Forecast errors for wind and PV were calculated by subtracting the real-time values from the day-ahead forecasts. All sites in each scenario were summed together, so the analysis provides an interconnection-wide evaluation of the forecasts. The real-time time series has 5 minute resolution, so the forecast error analysis averaged the 5-minute intervals within each hour to create an hourly resolution time series to compare to the hourly resolution day-ahead time series.

The forecast accuracy metrics of mean absolute error (MAE), root-mean-square error (RMSE), and mean error or bias, for the wind, PV, and total VG are presented below. MAE is the mean of the absolute value of the error calculated at each forecast point. This method equally weights all errors while the RMSE weights the individual errors by the size of the error. The RMSE metric emphasizes the extreme errors more than the MAE. The bias or mean error indicates if the errors are evenly distributed around zero. The PV forecast error is only calculated for daylight hours so nighttime hours (when PV output is known to be zero) do not artificially depress the error metrics.

Table 27 through Table 29 show the forecast error statistics for the wind, PV, and total VG at the interconnection level. In addition to the raw error statistics, the errors are also shown normalized by the installed capacity in each scenario.

Table 27 shows the error data for wind forecasts. As wind capacity increases, the absolute error metrics increase while the percentage error metrics generally decrease, suggesting that errors in opposite directions tend to offset each other. This is because forecast errors in particular locations will offset opposite-direction forecasts errors in other locations. The bias values seen in the wind forecast error data are negligible.

Table 27. Summary of U.S. EI Wind Forecast Error

\begin{tabular}{|l|r|r|r|r|r|r|r|}
\hline Forecast & Capacity & \multicolumn{2}{|c|}{ MAE } & \multicolumn{2}{|c|}{ RMSE } & \multicolumn{2}{c|}{ BIAS } \\
\hline & (MW) & (MW) & (\%) & (MW) & \multicolumn{1}{c|}{$(\%)$} & (MW) & \multicolumn{1}{c|}{$(\%)$} \\
\hline LowVG & 23,778 & 1,369 & $5.8 \%$ & 1,695 & $7.1 \%$ & -7 & $-0.03 \%$ \\
\hline RTx10 & 104,299 & 5,157 & $4.9 \%$ & 6,399 & $6.1 \%$ & -140 & $-0.13 \%$ \\
\hline RTx30 & 183,191 & 8,355 & $4.6 \%$ & 10,389 & $5.7 \%$ & -599 & $-0.33 \%$ \\
\hline ITx30 & 222,206 & 10,492 & $4.7 \%$ & 13,020 & $5.9 \%$ & -281 & $-0.13 \%$ \\
\hline
\end{tabular}

Table 28 shows the PV forecast error summary. PV forecast errors were only calculated for daylight hours. The same geographic diversity trends seen in the wind MAE and RMSE can be seen in the PV data. As capacity increases, the percentage MAE and RMSE decrease. The forecasts show a small positive bias. 
Table 28. Summary of U.S. EI PV Forecast Error

\begin{tabular}{|c|c|c|c|c|c|c|c|}
\hline \multirow[t]{2}{*}{ Forecast } & \multirow{2}{*}{$\begin{array}{l}\text { Capacity } \\
\text { (MW) }\end{array}$} & \multicolumn{2}{|c|}{ MAE } & \multicolumn{2}{|c|}{ RMSE } & \multicolumn{2}{|c|}{ BIAS } \\
\hline & & $(\mathrm{MW})$ & $(\%)$ & (MW) & (\%) & $(\mathrm{MW})$ & (\%) \\
\hline LowVG & 970 & 32 & $3.3 \%$ & 48 & $5.0 \%$ & 4 & $0.39 \%$ \\
\hline RTx10 & 5,016 & 160 & $3.2 \%$ & 251 & $5.0 \%$ & 10 & $0.21 \%$ \\
\hline RTx30 & 218,687 & 3,974 & $1.8 \%$ & 6,991 & $3.2 \%$ & 408 & $0.19 \%$ \\
\hline ITx30 & 109,806 & 2,045 & $1.9 \%$ & 3,531 & $3.2 \%$ & 301 & $0.27 \%$ \\
\hline
\end{tabular}

Table 29 shows the error statistics for the total VG production for the four study scenarios. Except for the bias ${ }^{21}$, the error statistics for total VG are not sums of the individual wind and PV components because concurrent wind and PV errors can offset and reduce the total error. The total VG percentage error metrics decrease more than the individual wind or PV metrics, indicating that errors in forecasts between the two technologies partially offset each other.

Table 29. Summary of Total U.S. EI VG Forecast Error

\begin{tabular}{|l|r|r|r|r|r|r|r|}
\hline Forecast & Capacity & \multicolumn{2}{|c|}{ MAE } & \multicolumn{2}{c|}{ RMSE } & \multicolumn{2}{c|}{ Bias } \\
\hline & (MW) & (MW) & $\mathbf{( \% )}$ & $\mathbf{( M W )}$ & $\mathbf{( \% )}$ & $\mathbf{( M W )}$ & \multicolumn{1}{c|}{$(\%)$} \\
\hline LowVG & 24,748 & 1,372 & $5.5 \%$ & 1,697 & $6.9 \%$ & -5 & $-0.02 \%$ \\
\hline RTx10 & 109,315 & 5,165 & $4.7 \%$ & 6,410 & $5.9 \%$ & -134 & $-0.12 \%$ \\
\hline RTx30 & 401,878 & 9,048 & $2.3 \%$ & 11,730 & $2.9 \%$ & -334 & $-0.08 \%$ \\
\hline ITx30 & 332,012 & 10,692 & $3.2 \%$ & 13,351 & $4.0 \%$ & -86 & $-0.03 \%$ \\
\hline
\end{tabular}

The distribution of forecast errors can offer some additional insight. The following figures show the distributions for wind, PV, and total VG for the two $30 \%$ penetration scenarios for the entire EI. The distributions are shown in two forms, nominal and normalized. The normalized distributions are generated by normalizing the error to the installed capacity of wind, PV, or total VG in each scenario. The nominal distributions are useful in understanding the magnitude and frequency of errors. The normalized distributions allow evaluation of how much the installed capacity versus other factors such as geographic diversity are driving the error distributions.

Figure 24 shows the distribution of day-ahead wind forecast errors for the two $30 \%$ penetration scenarios. In the nominal (non-normalized) distribution, the ITx30 shows a wider distribution, which is expected since it has more installed wind capacity than the RTx30. However, when normalized, the distributions are nearly identical, which indicates that (at the interconnectionlevel) there is no clear benefit of geographic diversity of one scenario versus the other.

\footnotetext{
${ }^{21}$ The total VG bias can be calculated as the sum of the average wind bias and the average all-hours solar bias. The bias average in Table 29 is not the simple sum of the biases in Table 27 and Table 28 because Table 28 excludes the night-time hours when solar forecasts are perfect but uninteresting.
} 

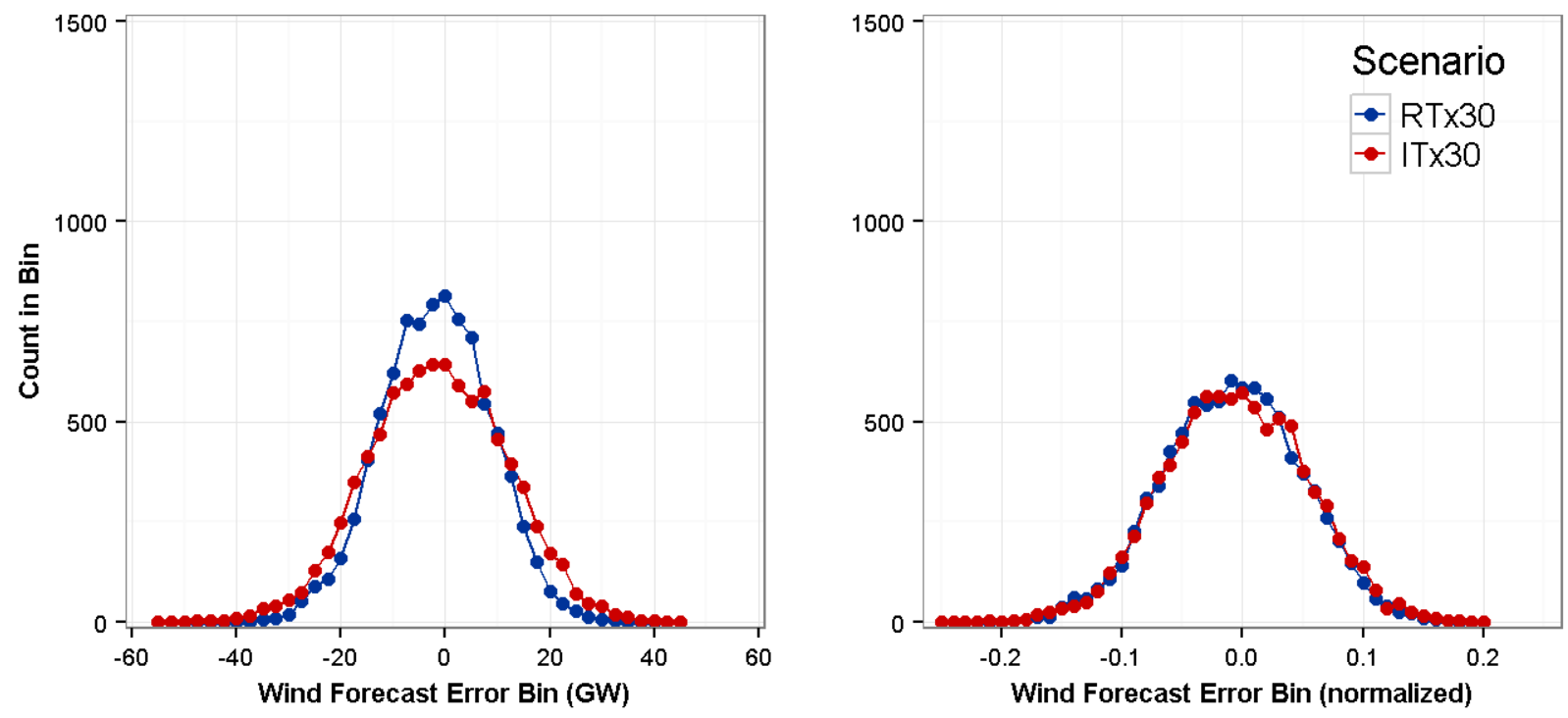

Figure 24. Distribution of day-ahead wind forecast errors. Bins have widths of 2.5GW (left-hand plot) and 0.01 (right-hand plot).

Figure 25 shows the distribution for day-ahead PV forecast errors for the $30 \%$ penetration scenarios. The statistics indicated a positive bias, which is verified by the shift of the distributions to the right on the graphs. For PV, there is more capacity in the RTx30 compared to the ITx30, which is consistent with the wider distribution of errors seen in the RTx30. Again, the normalized distributions are similar particularly in the tails. Compared to the wind distributions, the nominal PV distributions are somewhat narrower (due to lower installed capacity) and show a distinct peak near zero due to the large number of small errors in hours with low PV production (near sunrise and sunset).
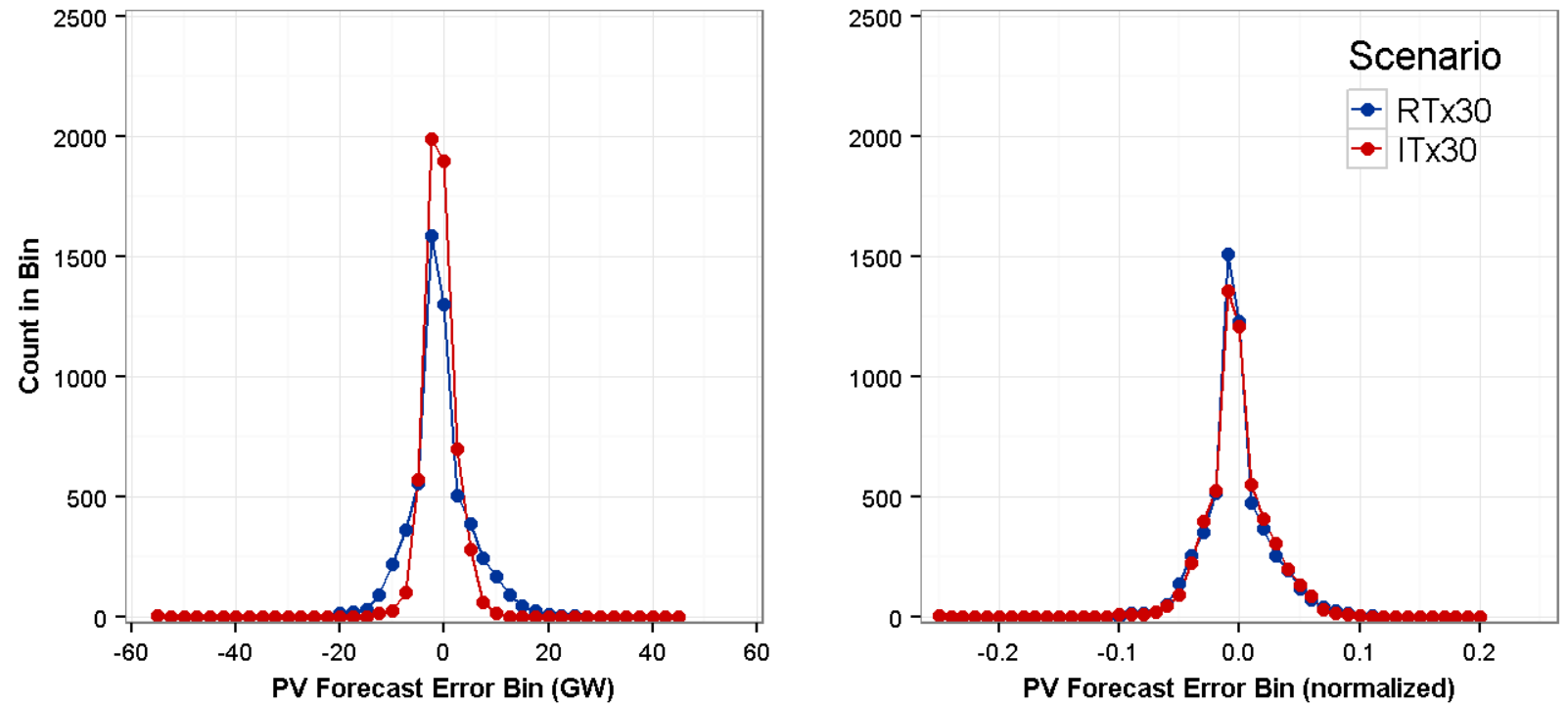

Figure 25. Distribution of day-ahead PV forecast errors. Bins have widths of 2.5GW (left-hand plot) and 0.01 (right-hand plot). 
The error distributions for total VG in the U.S. EI are shown in Figure 26. The nominal distributions are similar, with the ITx30 being slightly broader. The normalized ITx30 is significantly broader than the RTx30 because the absolute error distribution is broader and also because the total installed VG capacity in the RTx30 is about $21 \%$ larger than the ITx30.
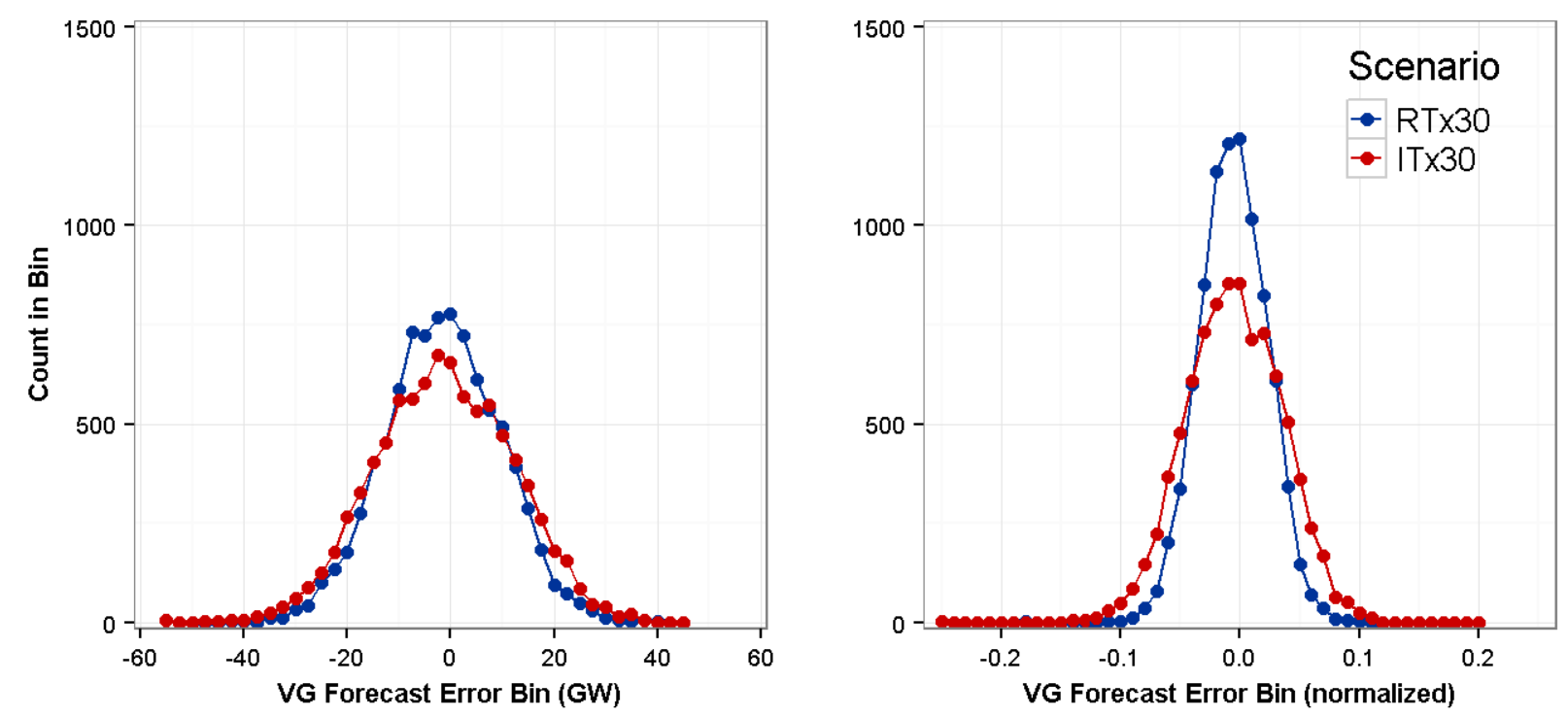

Figure 26. Distribution of total VG forecast errors. Bins have widths of 2.5GW (left-hand plot) and 0.01 (right-hand plot).

Average forecast error varies by time of day. Figure 27 shows the average daily wind, PV, and total VG forecast error profiles for the RTx30 and ITx30. The wind profile shows a positive peak (over-forecast) in the morning and a negative peak (under-forecast) in the evening. The PV profile shows under-forecasting in the morning and over-forecasting during the afternoon, in the RTx30. In the ITx30, shows a small under-forecasting bias. The total VG forecast profile is dominated by wind forecast errors in both scenarios. For reference the average load over the year is about $373 \mathrm{GW}$, so the peak average forecast error of about $5 \mathrm{GW}$ is about $1 \%$ of the average load. 

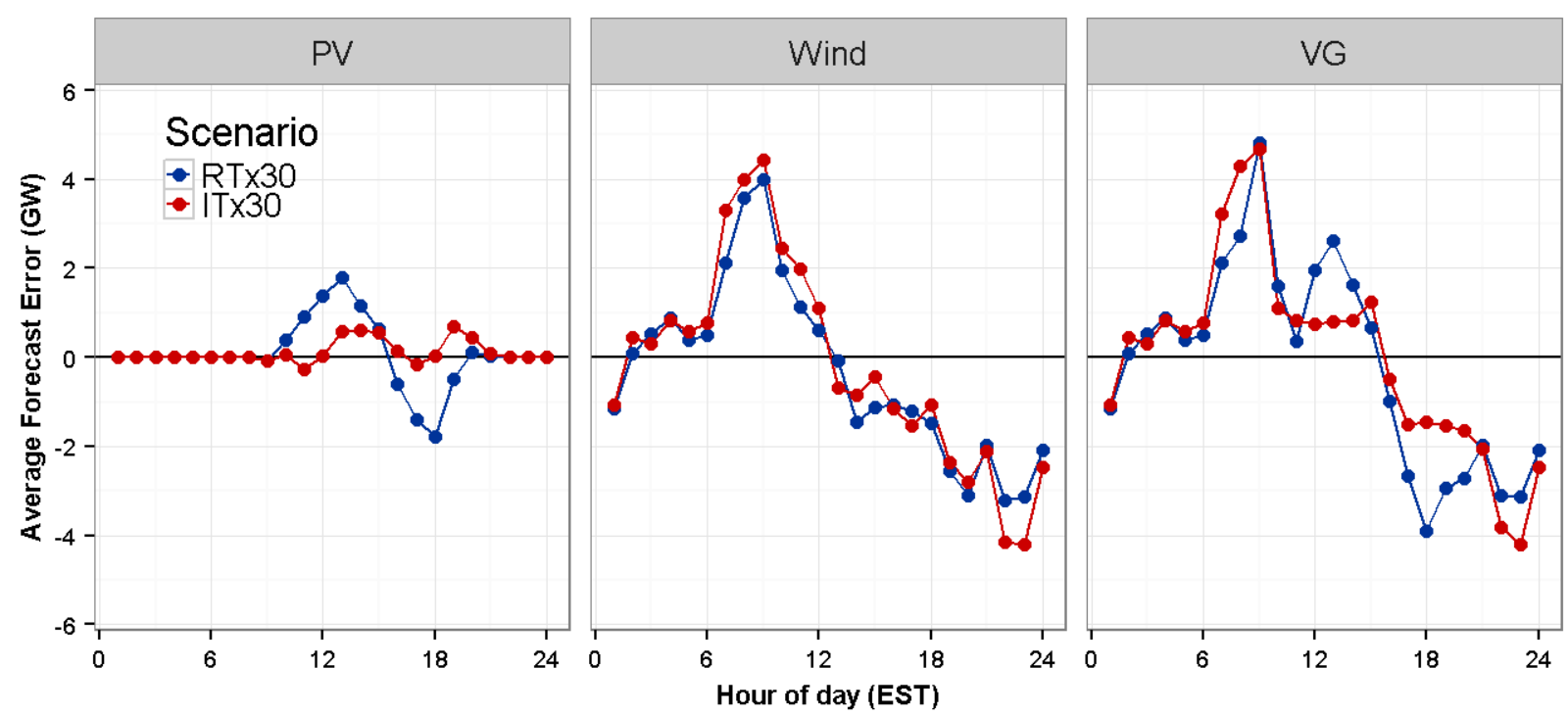

Figure 27. Average daily forecast error profiles for RT×30 and ITx30.

This section described the forecast error inherent in the input data for the different ERGIS VG scenarios. Due to an error while processing the input files for the PLEXOS model, the dayahead PV forecasts were made to be in their respective local times, rather than all being in Eastern Standard Time as the PLEXOS model expected. For example, a PV site in Florida was forecasted to begin generating at the same time as a PV site in western Nebraska, which is in the Mountain time zone. In reality, the western Nebraska site would not begin to generate until two hours after the Florida site. This lead to a shift in the PV forecast, exacerbating the day-ahead forecast error at all hours of PV generation. Depending on the location of the PV site, this could mean the forecast was consistently under-forecasting PV generation in the morning, while overforecasting in the evening. The real-time profiles, however, were correctly implemented in Eastern Standard Time, so all modeled results of the system dispatch presented throughout this document use the correct data. The impact of the increased PV forecast error, caused by the time zone error in the day-ahead forecasts, is to create a more conservative day-ahead unit commitment. This would tend to slightly overstate the costs of operating a system with VG, or slightly understate the operational benefits of high VG penetration. 


\section{Reserve Calculation}

\subsection{Operating Reserves}

Operations of power systems occur at a range of timescales that can be summarized, from longer to shorter, as unit commitment, load-following, and regulation (Ela et al. 2011). Unit commitment and scheduling are performed to economically commit the units in the system to meet forecasted load and other system requirements. During shorter periods of time (minutes to hours), the system redispatches its units to counteract deviations from the schedule through load-following. Similarly, some units are controlled to perform regulation, which is the fast response of generators to changes that range from seconds to minutes.

The operator requires operating reserves so the system can positively respond to forecast errors and events that cannot be accounted for in the scheduling process. In the United States, the most common are regulation reserve and contingency reserve. These reserves are designed to account for the system's variability (expected changes) and uncertainty (unexpected changes). Both load and conventional generators also cause a need for reserves through forecast errors and unexpected outages, respectively.

Regulation reserves are used to accommodate second-to-second and minute-to-minute changes in net load, driven by the combination of all changes in electricity supply and demand. Contingency reserves are used to accommodate unexpected failures of power system components, typically the failure of a large conventional generator that causes an abrupt and unexpected loss of supply.

All sources of supply and demand have some variability and uncertainty, and power system operators use reserves to accommodate these changes. Wind and PV are variable and uncertain in nature because their output depends on weather conditions that cannot be perfectly predicted ahead of time.

We determined the reserve requirements necessary to cover a significant level of uncertainty in wind and PV output (Ibanez et al. 2013). Only "up" reserve requirements (caused amongst other things by down-ramps from wind/PV) are simulated in ERGIS because we assumed that excessive energy from wind and PV could be curtailed if the system were unable to accommodate it.

\subsection{Reserve Sharing Regions}

The ERGIS reserve sharing regions were designed to approximate actual reserve sharing regions in the Eastern Interconnection. Two types of reserve sharing regions are used in ERGIS. Contingency reserves were held for each of the sub-regions identified in Figure 39. As discussed elsewhere, the ERGIS sub-regions reflect TRC input on intra-regional transmission constraints. Regulation reserves were procured at the regional level, and reflect 2010 sharing regions in the RTOs/ISOs. One notable difference between our modeled regulation regions and actual regions in the year 2014 can be found with SPP and MISO. As discussed previously, the upper northwest of the ERGIS MISO region became part of SPP in 2015. In the non-RTO/ISO regions, regulation reserves were procured on a sub-regional basis and reflect the 2010 borders of Southern Companies (SOCO), Duke Energy (Duke), the Tennessee Valley Authority (TVA), and FRCC. Regulating reserve regions are detailed Figure 28. 


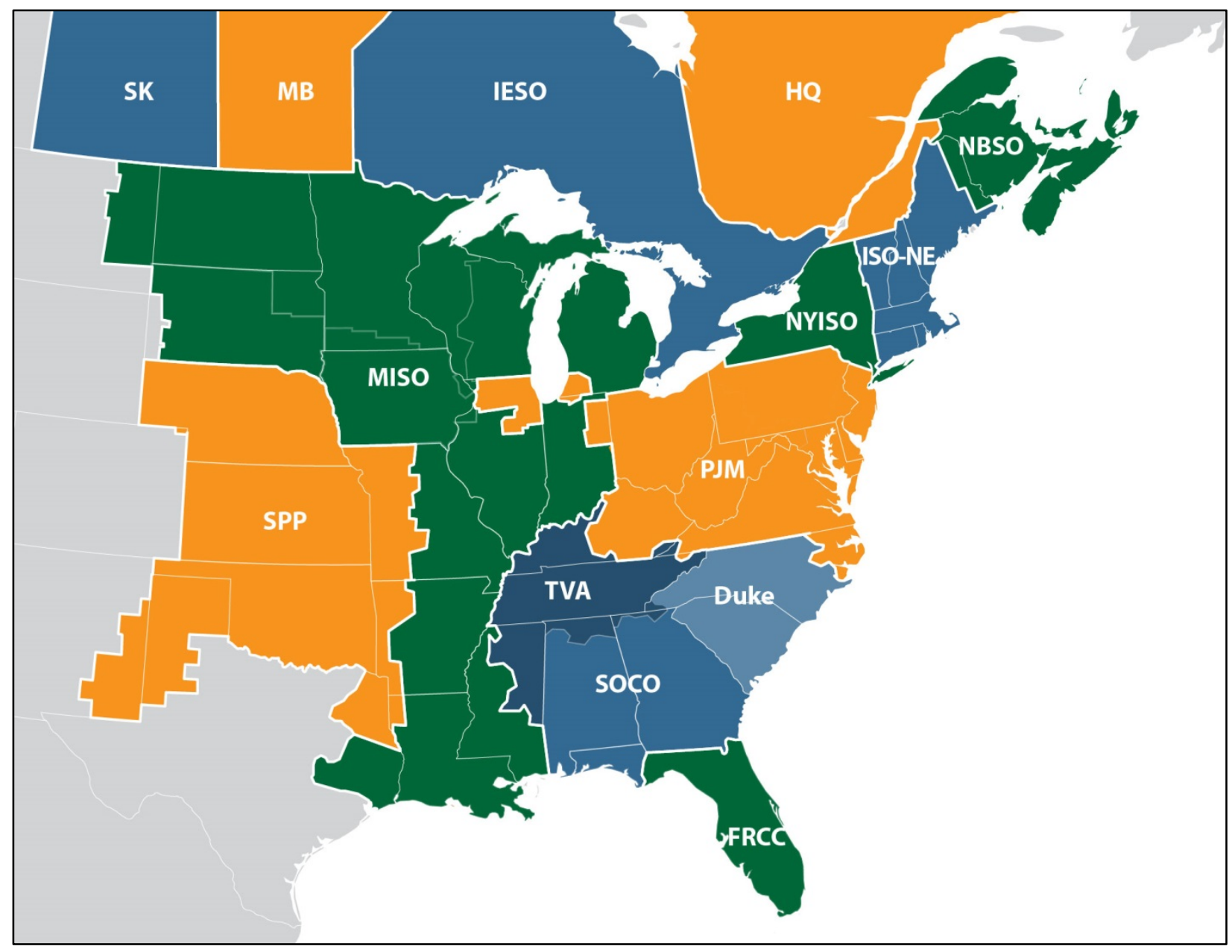

Figure 28. Regulation Reserve Sharing Regions

\subsection{Wind Reserve Methodology}

Previous work (Ela et al. 2011; EnerNex 2011) quantified how the uncertainty of wind power can be used to determine reserve requirement levels. Because short-term variations in wind power output are small, persistence forecasts (which assume the current level of output will be the level of output of a resource at some point in the near future) are a good predictor with which to calculate uncertainty. For instance, for an economic dispatch model run in 5-minute intervals (and assuming that 5 additional minutes are required to perform calculation and dispatch communication), 10-minute persistence forecasts would likely be used for scheduling. Thus, errors based on 10-minute persistence forecast and realized production can be used to estimate the uncertainty that the power system must be able to handle between dispatch points.

Figure 29 shows the forecast error versus power output for MISO for the ITx30 scenario. The figure shows that wind forecast errors are highest at moderate total wind production levels. Forecast errors can be positive or negative, and the wind turbine power curve is expected to be steepest at moderate wind speeds. These data are for the MISO footprint in the ITx30 scenario, but the behavior is similar across regions and scenarios. 
Confidence intervals (represented as red and blue lines) were used to determine the reserve requirements so that a certain percentage of forecast errors were covered by the reserve. In Figure 29, the range of power (horizontal axis) was divided in 10 groups with the same number of points in each group. For each group, the average power was calculated as well as the confidence intervals that covered $95 \%$ of the forecast errors. The confidence intervals bands were then interpolated from the group averages. Below the first group's mean point and above the last group's mean point, the requirements were kept constant as a simplified conservative approach. A higher number of groups or a more sophisticated method (e.g., a moving window across the cloud of points) would yield a better fit around the lower and upper power regions.

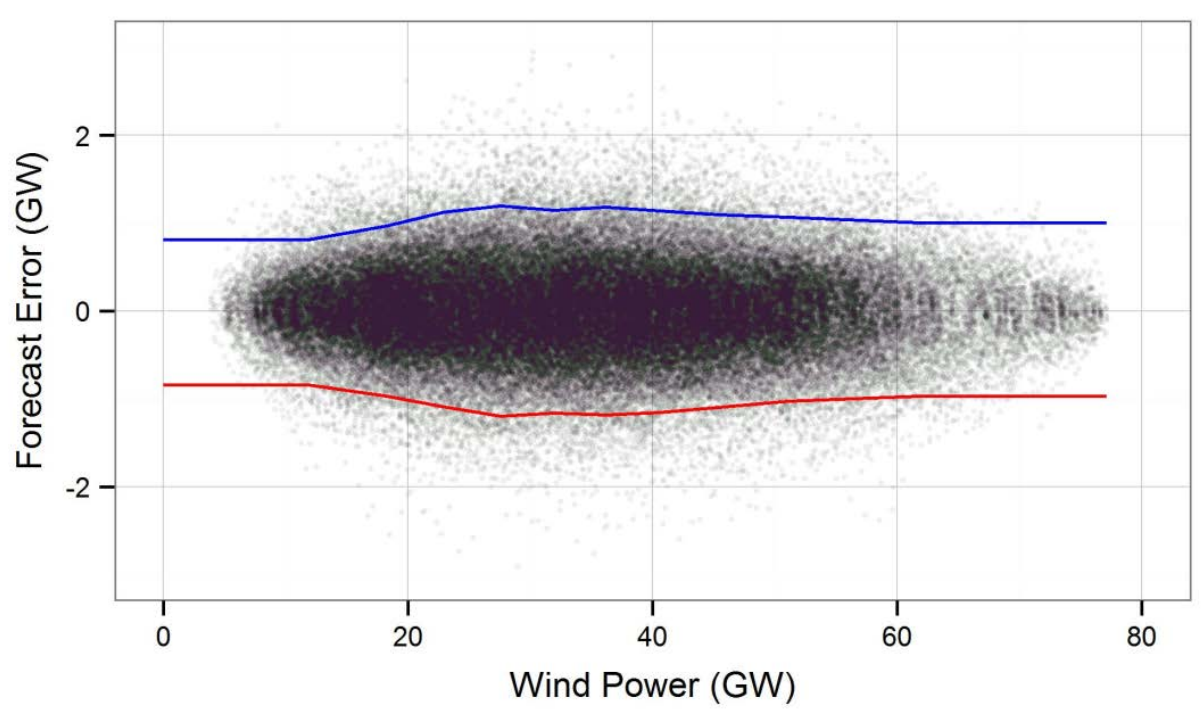

Figure 29. Wind 10-minute forecast errors versus power output, with $95 \%$ confidence interval bands for MISO in the ITX30 scenario

The regulation reserve requirements were based on 10-minute persistence forecasts and confidence intervals that covered $95 \%$ of the forecast errors. These requirements approximate levels of coverage used in past integration studies (EnerNex, 2011; Lew et al., 2013). ${ }^{22}$

The result of this analysis was a dynamic determination of reserve requirements in which the reserve requirement for each region and each hour of the year is a function of the forecast wind power output, as illustrated in Figure 30.

\footnotetext{
${ }^{22}$ The Eastern Wind Integration and Transmission Study (EnerNex 2011) assumed that spinning reserve covered one standard deviation of the 1-hour ramps. That coverage approximates to $70 \%$ under a normal distribution.
} 

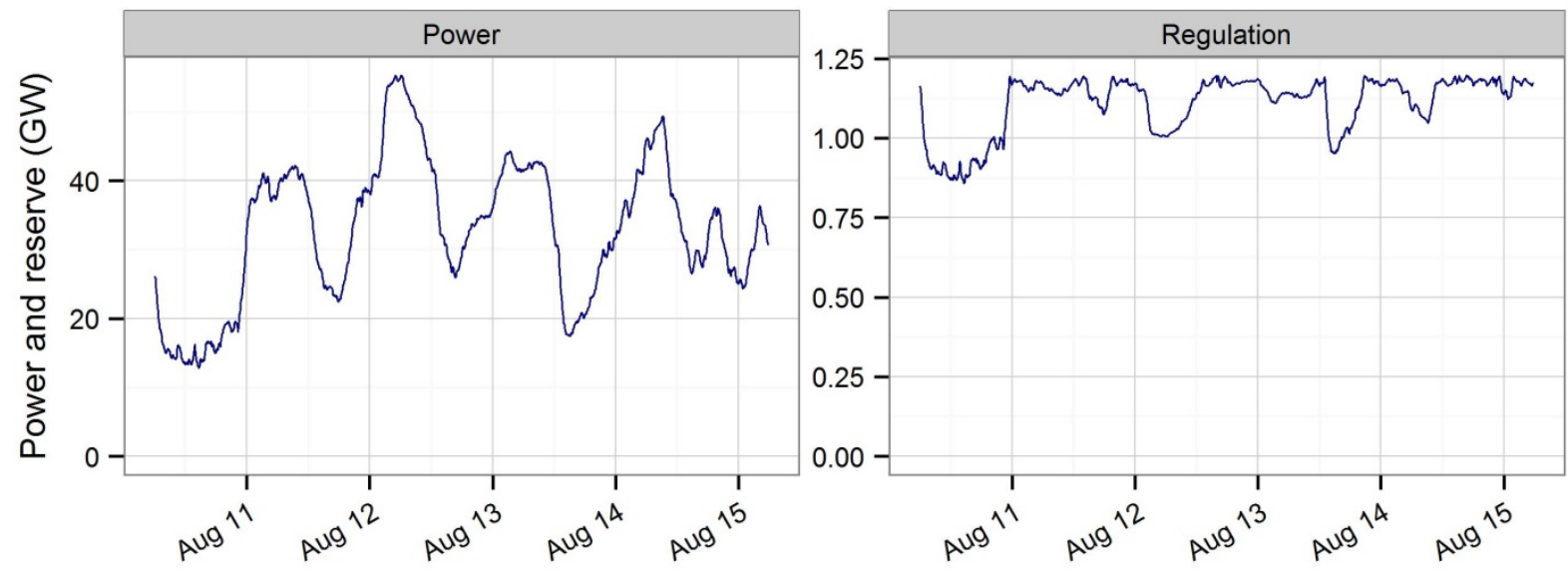

Figure 30. Wind power production and dynamic regulation reserve requirement for MISO in the ITx30

Note: The y-axis scales differ.

\subsection{PV Reserve Methodology}

The PV reserve methodology builds on the wind methods previously presented. Some adjustments were necessary to take into account PV daily patterns, but the process (Ibanez et al. 2013) follows three distinct steps: (1) defining forecast error; (2) using explanatory variables to group similar patterns; and (3) applying the reserve requirements based on the explanatory variables.

The wind forecast errors were calculated based on persistence forecasts. Power output was used as an explanatory variable to find reserve requirements (Figure 29) and to create the dynamic reserve requirements (Figure 30). The following sections develop similar concepts for PV power.

PV-based generation presents clear patterns because of its dependence on the sun's path across the sky. These patterns are best captured with clear-sky simulations, which calculate the power output in the absence of clouds. The left panel in Figure 31 represents the actual and clear-sky power outputs in the MISO during four summer days in the ITx30 scenario. The right panel represents the 10-minute ramps in the same timescale. 


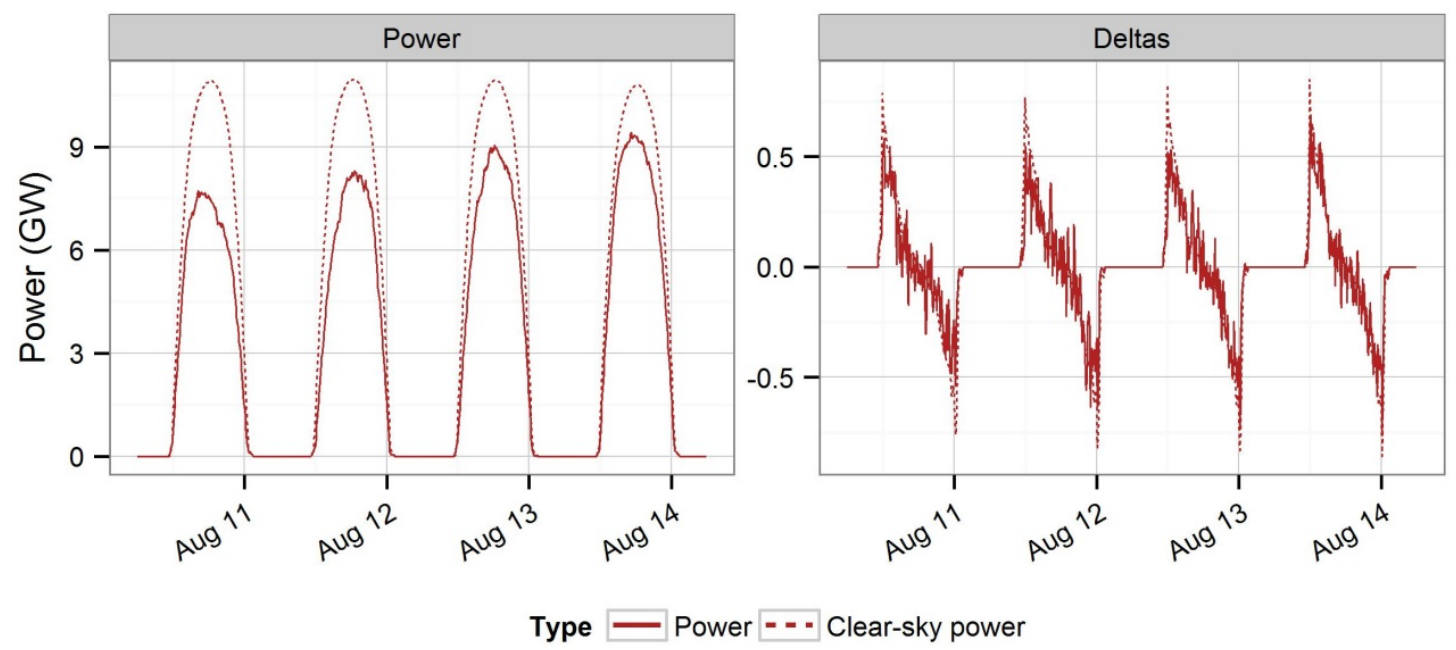

Figure 31. Power and clear-sky power output and ramps for MISO in the ITx30 scenario

If the same power-persistence forecast used for wind had been applied in this case, the largest demand for reserve would have occurred consistently around sunrise and sunset and the smallest during the middle of the day. However, reserves are not needed to cover known changes in power output because this is considered in the hourly and 5-minute unit commitment through the use of PV forecasts. Figure 31 shows that the power deltas could be decomposed into the contribution from the clear-sky power and a smaller, high-frequency variation because of weather. In other words, if the clear-sky trends (which are known) were removed from the power deltas, the reserve requirements would be smaller.

The first step in the creation of the short-term PV forecast was the definition of the PV power index (SPI), which represents the ratio between actual power, $P$, and clear-sky power, $P_{C S}$.

The forecast was then based on the persistence of SPI. To obtain the forecast, we added the clearsky ramp scaled by the SPI to the current power output. An example of PV forecast error can be found in Figure 32. The forecast error could then be calculated as:

$$
\operatorname{Error}(t)=P(t+1)-\left\{P(t)+S P I(t) \times\left[P_{C S}(t+1)-P_{C S}(t)\right]\right\}
$$




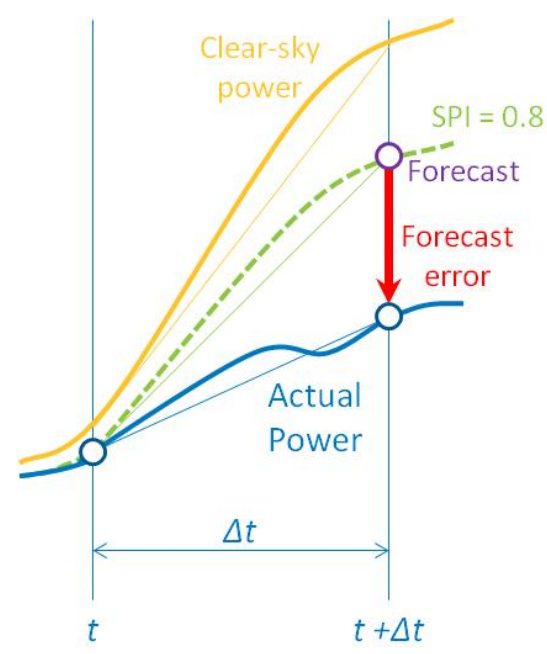

Figure 32. Graphical representation of short-term forecast for PV

Early morning forecast errors tended to be among the largest because clear-sky values are either zero or very small, which in turn make the SPI values non-existent or very unstable. At the same time, PV power usually increases rapidly. During these times we chose to use a 24-hour persistence of power for the forecasts (i.e., taking the values from the previous sunrise as forecasts).

Figure 33 shows the effect of using the clear-sky information to correct the short-term forecasts. The SPI persistence forecast has a much narrower distribution than the simpler powerpersistence forecast. The standard deviation for the two distributions is 105 and $279 \mathrm{MW}$, respectively. A more accurate short-term forecast ultimately leads to a significant reduction in reserve requirements. Clear-sky ramps will be met through the commitment of units with the remaining variability met through increased reserve, similar to the way that dispatch meets the forecasted load ramps and regulation is used only to meet load variations outside the forecasted ramps from one interval to the next.

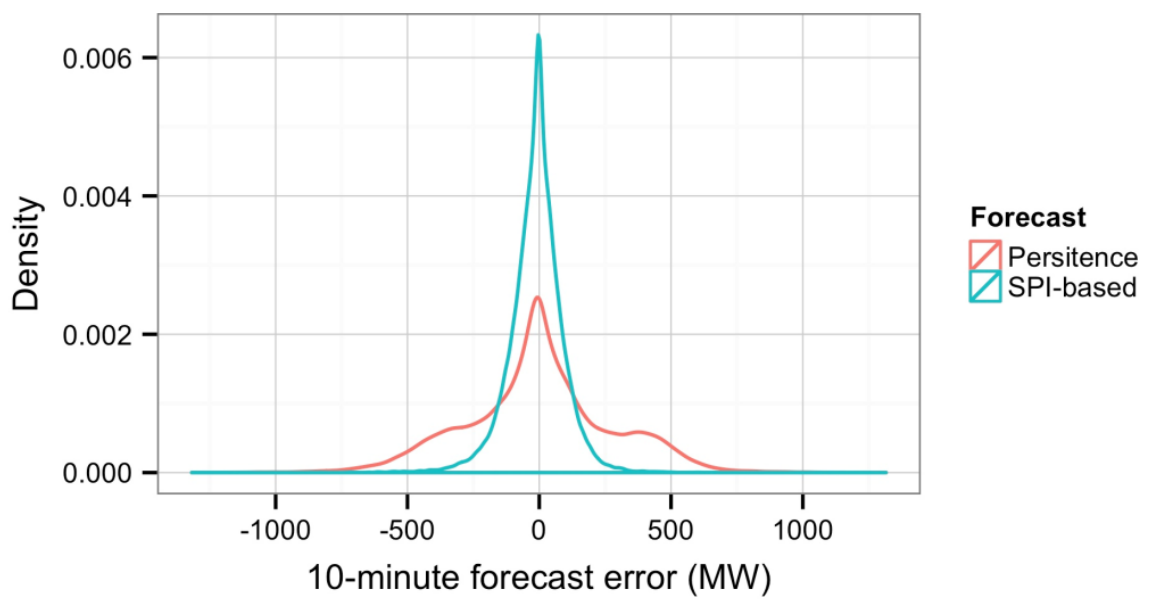

Figure 33. Ten-minute PV forecast error distribution for the persistence and the SPI-based methods for MISO in the ITx30 scenario 
In order to understand similarities between events, a classification method was necessary. We found that the following two explanatory variables were effective at classifying events present in the study datasets:

- SPI, as defined in the previous subsection, which effectively separated "cloudy" and "sunny" days

- Clear-sky ramps, which separated the different times of the day (e.g., positive in the morning, close to zero at midday, and negative toward sunset).

For ERGIS, we calculated the reserve based on the 5-minute time series. We first created 10 divisions of each explanatory variable, forming 100 groups by combining both variables. For each group, we calculated the reserve by taking the appropriate confidence intervals (e.g., the 2.5 and 97.5 percentiles to create $95 \%$ confidence intervals). To prevent outliers from dominating the reserve, we did not calculate reserve requirements for a group if it presented less than 20 members. In that case, we used the reserve for the closest group instead.

We applied this method to all scenarios and reserve-sharing regions in ERGIS. We calculated regulation reserve using 10-minute time steps and 95\% confidence intervals for the entire footprint. The results are represented in Figure 34, which suggests that reserve requirements depend on the combination of the explanatory variables, SPI and clear-sky ramps. The highest down-reserve requirements were usually located on the top right corner, which corresponded to sunrises where SPI was close to 1 . On such occasions, the calculation of SPI was highly unstable given that the denominator (clear-sky power, $\mathrm{P}_{\mathrm{CS}}$ ) was very small, as previously mentioned. At times, the forecast called for a "sunny" sunrise, and the clear-sky correction was heavily weighted in the error calculation. The inability of this method to produce a good forecast for these particular instances created the high reserve requirements.

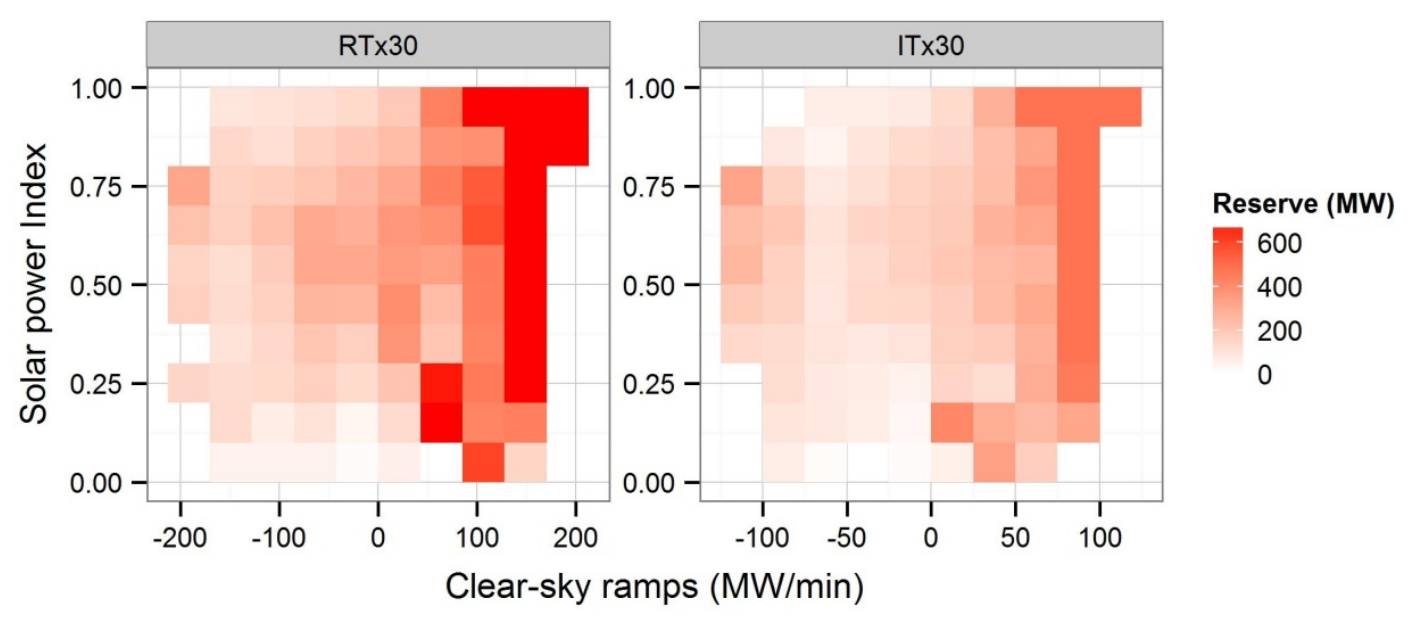

Figure 34. PV component of the regulation reserve requirements for the RTx30 and ITx30 scenarios for MISO

After the reserve requirements were determined, they could be applied to the time series data for PV. For example, Figure 35 represents the resulting requirements for regulation reserve from PV in the MISO for the ITx30 scenario during a few selected days in August. 

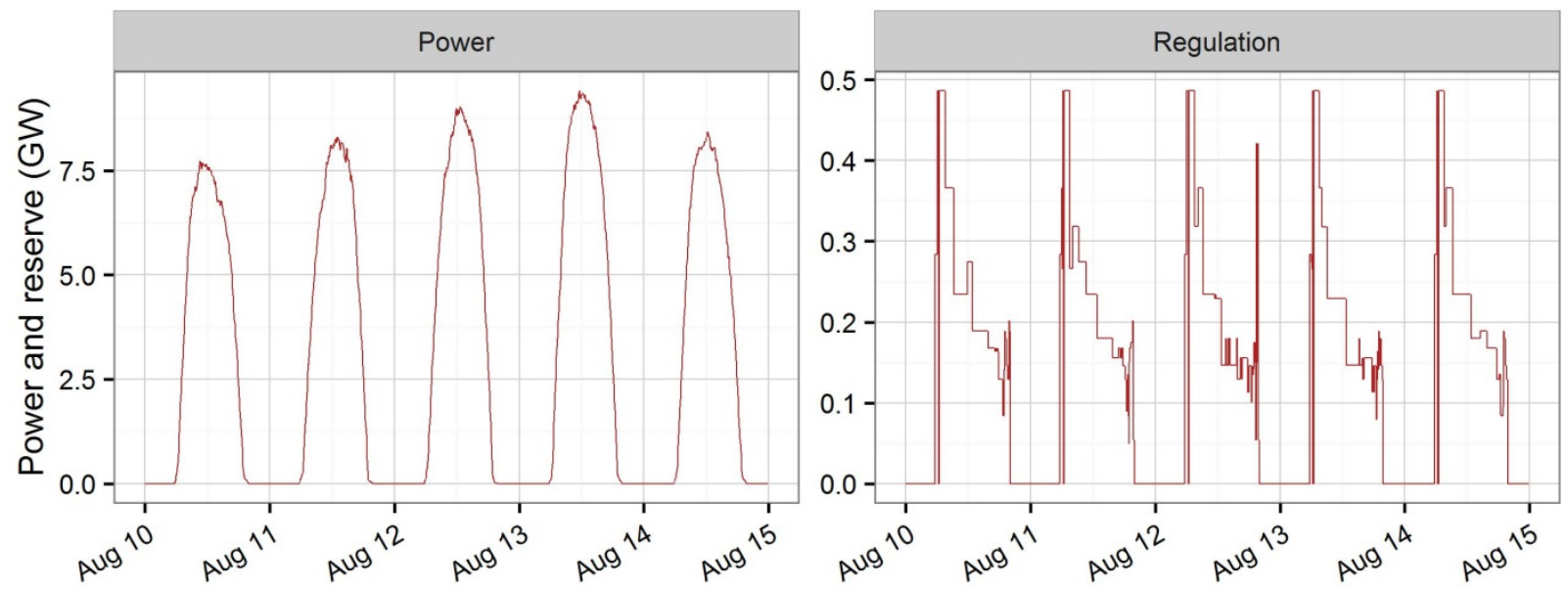

Figure 35. Dynamic PV regulation reserve requirements for MISO for the ITx30 scenario

\subsection{Total Reserve Requirements}

The reserve methodologies for wind and PV were used along with the base requirements for contingency and load-only regulation reserve, as determined in Lew et al. (2013). These two types of spinning reserve were considered in this study and held for each reserve-sharing region independently. The total requirements were calculated as follows:

- Contingency reserve: $3 \%$ of the load with no consideration of wind or PV

- Regulation reserve: Geometric sum of base requirement (1\% of load) and contribution of wind and PV (which cover 95\% of 10-minute forecast errors).

The different requirements were added geometrically given that for short time steps, the forecast errors were considered to be uncorrelated. Table 30 summarizes the requirements for spinning reserve. Non-spinning reserve products were not modeled in the study.

Table 30. Requirements for Spinning Reserve

\begin{tabular}{l|c} 
Reserve & Calculation \\
\hline Contingency & $3 \%$ load \\
\hline Regulation & $\sqrt{(1 \% \text { load })^{2}+(\text { Wind rqt })^{2}+(P V \text { rqt })^{2}}$ \\
\hline
\end{tabular}

Table 31 presents the day-ahead amounts of reserve required for each scenario for the entire study footprint, including Canada. Contingency reserves remain unchanged because it is based on a percentage of load, and is intended to accommodate system contingencies, which do not change in this study. Total regulation reserve increases slightly for the RTx10 scenario and much more for the high-penetration scenarios. In general, higher penetrations of wind (e.g., in the ITx30 scenario) tend to increase the average and total demand for reserve, and higher penetrations of PV increase the maximum demand for reserve. 
Table 31. Annual Amounts of Reserves

\begin{tabular}{|l|c|c|}
\hline Scenario & Contingency (TW-h) & Regulation (TW-h) \\
\hline LowVG & 97.1 & 37.3 \\
\hline RTx10 & 97.1 & 43.5 \\
\hline RTx30 & 97.1 & 55.8 \\
\hline ITx30 & 97.1 & 56.9 \\
\hline
\end{tabular}

Notably, at 30\% renewable penetrations, the annual need for contingency reserves is still nearly twice as large as the annual need for all regulation reserves using our methodology. Moreover, the incremental contribution of renewable resources is roughly only $1 / 3$ of the total need for regulation, as indicated by the 17-18 TW-h increase in regulation reserve needs in moving from the LowVG case to the $30 \%$ renewable cases. As a result, the total incremental reserves needed to reach $30 \%$ renewable generation is less than $20 \%$ of the amount of contingency reserves that are primarily held to accommodate the unexpected outages of conventional generators and transmission facilities.

Figure 36 combines the wind and PV power time series with regulation and reserve requirements for a few selected days in August for the MISO footprint. The total reserve levels are represented, along with the load, wind, and PV components. The geometric addition causes regulation requirements to be dominated by the load component in the LowVG scenario, while the PV and (especially) wind components have a larger impact in the high-penetration scenarios. For the most part the wind component of reserve tends to contribute more evenly throughout the day, explaining the overall higher requirements. PV tends to fluctuate throughout the day. 

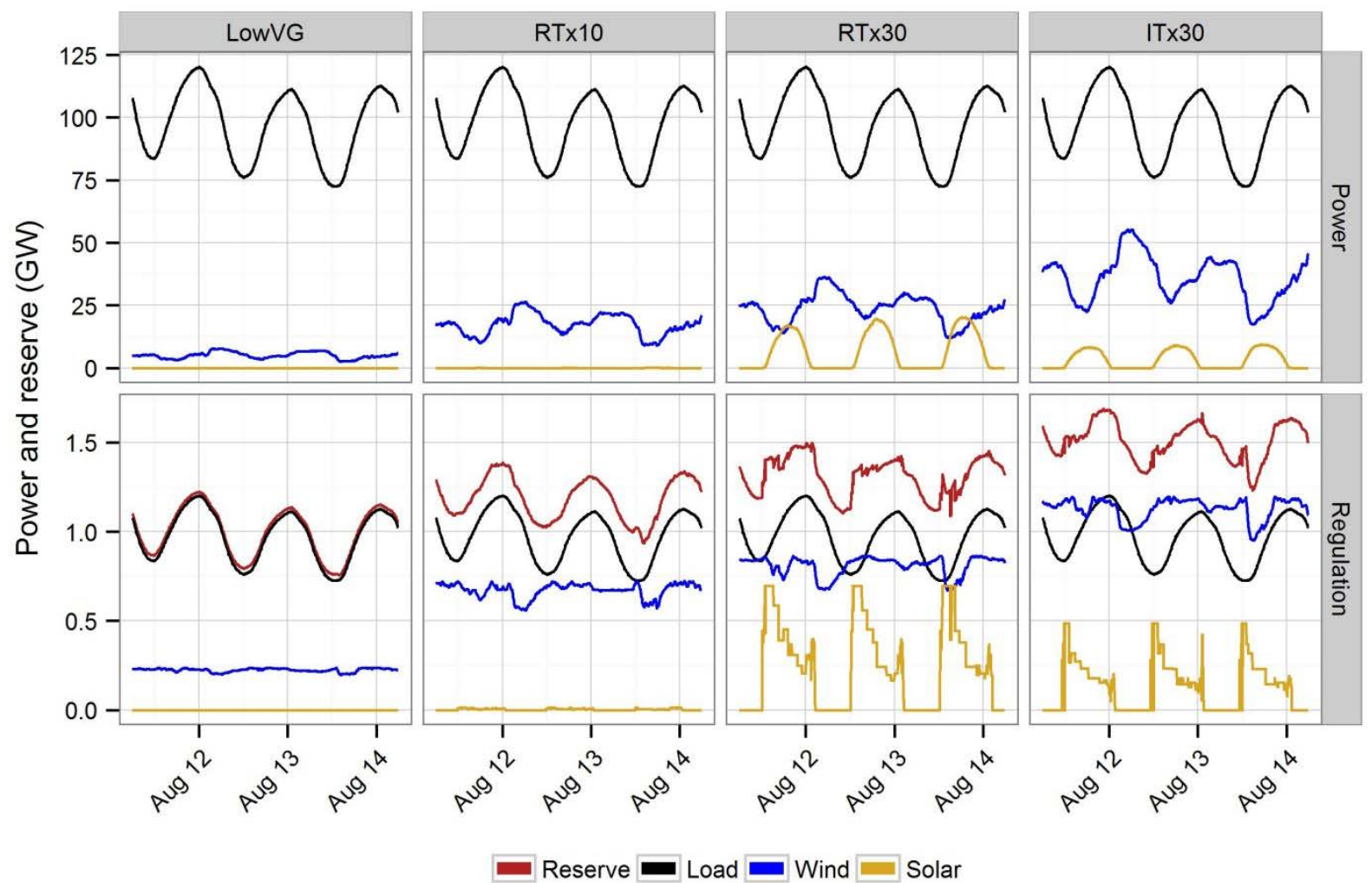

Figure 36. Power, regulation reserve for a few selected days in August, for MISO, by scenario

\subsection{Reserve Requirements by Region}

This subsection examines regulation reserve requirements for each reserve-sharing region in the United States. Total day-ahead regulation reserve requirements are presented in Table 32, while Figure 37 contains a graphical representation of the distribution by scenario.

Both types of reserve present similar trends. A large amount of wind in a region tends to raise the requirement evenly, while the presence of $\mathrm{PV}$ increases the maximum values along with the width of the distribution. Regions with little or no presence of renewables have regulation reserve distributions that mostly depend on the load component. There are marked differences in reserve requirements between the two high-penetration scenarios, which are largely driven by the location and installed capacity for wind and PV. 
Table 32. Regulation Reserve Requirements by Region (TW-h)

\begin{tabular}{|l|r|r|r|r|}
\hline & LowVG & \multicolumn{1}{|c|}{ RTx10 } & \multicolumn{1}{|c|}{ RTx30 } & \multicolumn{1}{|l|}{ ITx30 } \\
\hline Duke & 2.53 & 2.56 & 3.62 & 2.93 \\
\hline FRCC & 2.57 & 2.57 & 5.13 & 4.36 \\
\hline ISO-NE & 1.39 & 1.67 & 2.42 & 2.14 \\
\hline MISO & 8.02 & 9.63 & 10.70 & 12.63 \\
\hline NYISO & 1.71 & 2.56 & 2.76 & 2.83 \\
\hline PJM & 9.20 & 9.86 & 12.47 & 11.77 \\
\hline SOCO & 2.76 & 2.76 & 3.68 & 3.15 \\
\hline SPP & 2.70 & 5.46 & 7.75 & 10.54 \\
\hline TVA & 2.15 & 2.15 & 2.99 & 2.27 \\
\hline
\end{tabular}
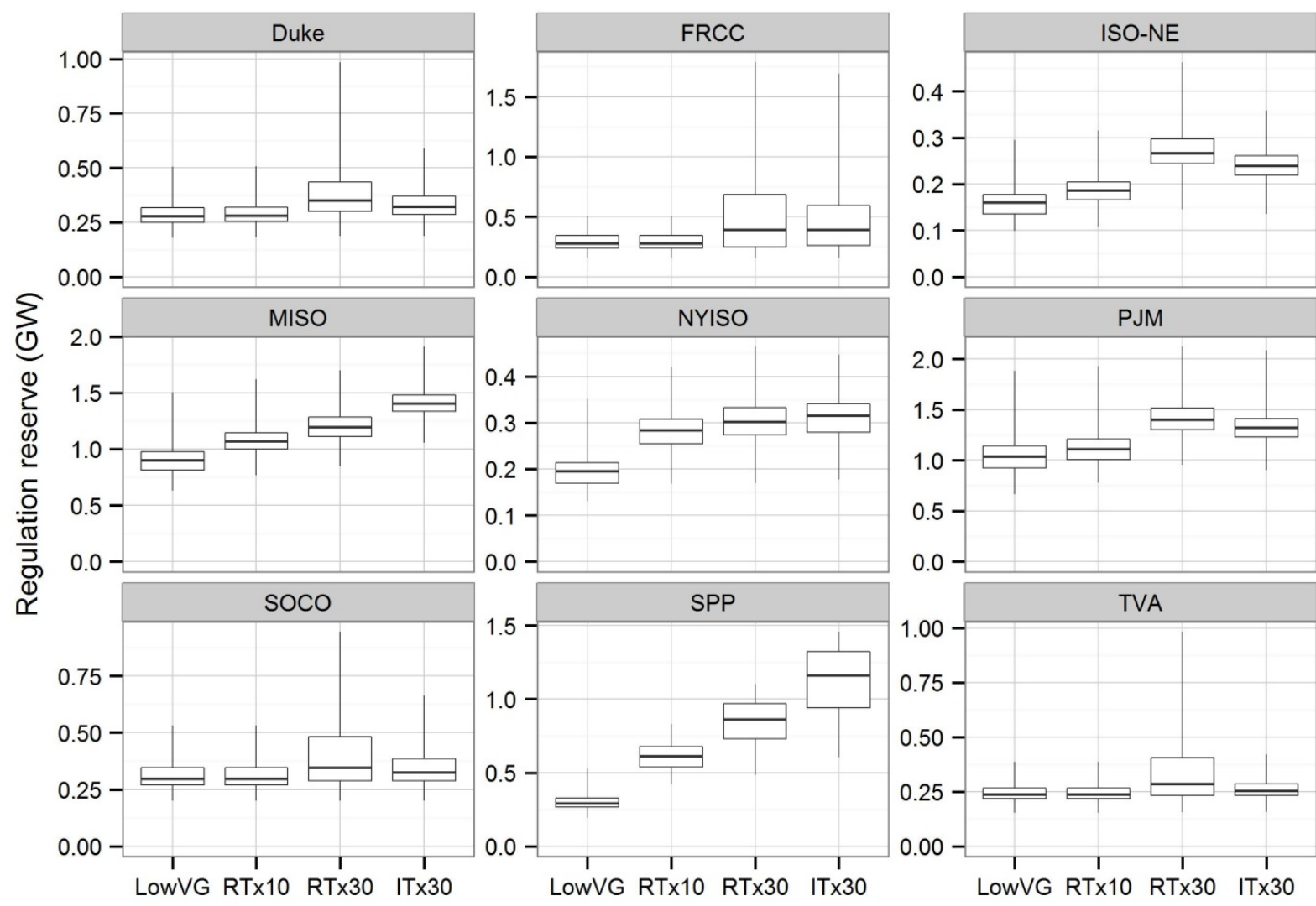

Figure 37. Distribution of regulation reserve by region and scenario Notes: The thick horizontal line is the median regulation reserve requirement. The box shows the 25th to 75th percentile of regulation reserves.

The lines show the extent of the maximum and minimum regulation capacity. 


\subsection{Reserve Sensitivity With Respect to Aggregation Level}

The determination of reserve-sharing regions was developed with the assistance of the TRC and is loosely based on current reserve-sharing regions. Although not executed in any of the actual model runs, this section explores the reduction in reserve requirements if five of the southernmost regions (which are amongst the smallest) were to be combined. As has been found in other NREL analyses, the need for operating reserves is drastically reduced when reserves are shared across a larger geographic footprint. This reduction is solely due to the geographic smoothing that happens for wind and PV profiles. ${ }^{23}$ Table 33 summarizes the aggregation levels considered, with all of the reserve-sharing regions considered for this section in the first column.

Table 33. Level of Aggregation Studied for Southern Regions

\begin{tabular}{|c|c|c|c|}
\hline $\begin{array}{c}\text { Level } \mathbf{1} \\
\text { (Regions) }\end{array}$ & Level 2 & Level 3 & Level 4 \\
\hline SPP & SPP & SPP & $\begin{array}{c}\text { SERC \& } \\
\text { SPP }\end{array}$ \\
\hline SOCO & SERC & Southeast & \\
\hline Duke & & & \\
\hline TVA & & & FRCC \\
\hline FRCC & FRCC & & \\
\hline
\end{tabular}

Table 34 summarizes the total day-ahead regulation reserve requirements for the regions considered at the various levels of aggregation. Similarly, Figure 38 shows duration curve for the regulation reserve requirements.

Table 34. Total Reserve Requirements for the Five Selected Regions at Different Aggregation Levels (TW-h)

\begin{tabular}{|c|c|c|c|c|}
\hline Scenario & Level 1 & Level 2 & Level 3 & Level 4 \\
\hline LowVG & 12.70 & 12.70 & 12.70 & 12.49 \\
\hline RTx10 & 15.50 & 15.47 & 15.47 & 13.61 \\
\hline RTx30 & 23.16 & 22.10 & 20.46 & 18.64 \\
\hline ITx30 & 23.25 & 22.76 & 21.76 & 18.87 \\
\hline
\end{tabular}

Given the small amount of wind and PV in the regions considered for the LowVG and RTx10 scenarios, there is little benefit from higher levels of aggregation. The only exception is the Level 4 aggregation for regulation reserve for the RTx10 scenario (a 12\% reduction when compared to its corresponding Level 1). This is due to the fact that the wind component reserve stays the same for SERC and SPP (most of the wind is installed in SPP), but the load component increases.

\footnotetext{
${ }^{23}$ As discussed in Section 6.5, the load component of regulation reserve is fixed to $1 \%$ and, thus, there is no benefit within that component when different regions are aggregated. Hence, there could be additional reductions in reserve if more complex rules were implemented (King et al. 2011).
} 
Level 4 (in which SPP, SOCO, TVA, and Duke are combined) also has the largest reductions in reserve requirements for high penetration scenarios (around a 20\% decrease in regulation when compared to the lowest aggregation level in RTx30). This reduction happens across all hours of the year by increasing the load footprint that shares the large amounts of wind installed in SPP.

Level 3 (in which SOCO, TVA, Duke, and FRCC are combined) shows small reductions in the ITx30 (a 6\% reduction in regulation) but larger reductions for the RTx30 (a 12\% reduction in regulation). This is because the latter sees a higher deployment of renewables (primarily PV) in those regions. The reductions for both high-penetration scenarios are concentrated on the upper half of the reserve distribution, where PV contributes the most.

In conclusion, large reserve-sharing regions reduce the total requirements, especially in high-VG penetration scenarios, mainly due to geographic diversity (King et al. 2012). These calculations assumed that load had a constant contribution to reserve requirements ( $1 \%$ for regulation). Thus, this reduction would be even greater due to reduction in overall load variability. However, it is important to note that this assumes enough transmission is available to ensure deliverability of reserve across a reserve-sharing group.

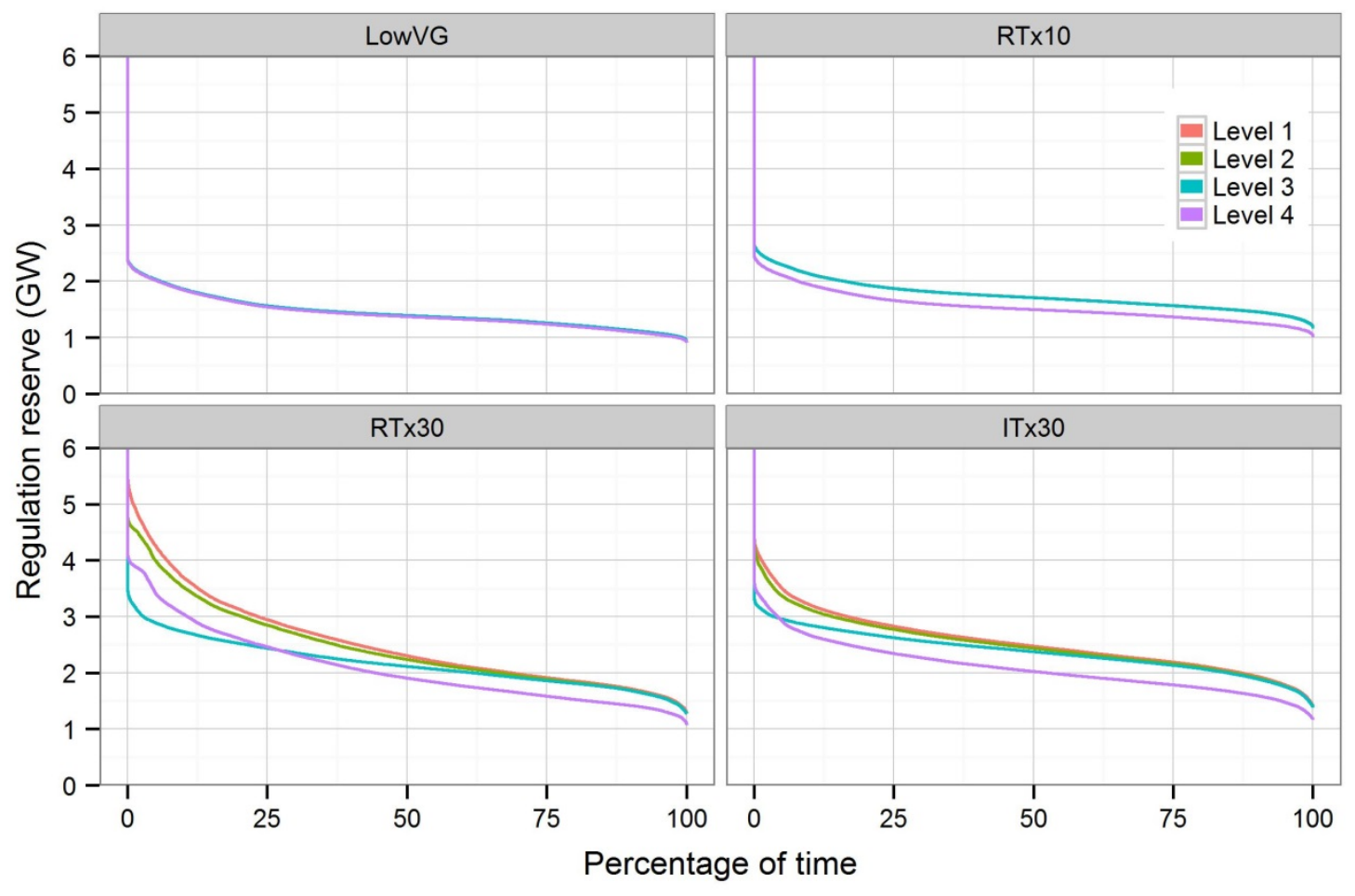

Figure 38. Regulation reserve duration curves by scenario and level of aggregation 


\subsection{Reserves Procurement and Comparisons}

The goal of the NREL reserve methodologies is to assure a statistically significant amount of the short term unpredicted variability in the wind and solar resource generation can be managed through the use of regulating reserves. In this section we compare a historical regulating reserves procurement summary from Denholm et al. (2015) to the amount of regulating reserves procured in the ERGIS simulations, Table 35. We also present the actual and simulated peak load conditions for each region.

As explained in Section 4.3.4, the ERGIS regions do not directly correspond to the actual RTO/ISO and non-RTO/ISO regional boundaries in 2016. Despite the difference between regional boundary definitions in ERGIS and the actual system in 2016, a comparison of the required reserve capacity in the ERGIS runs provides perspective on how much our modeling deviates from historical behavior.

Table 35. Peak Load and Capacity of Regulating Reserves Held in each ERGIS Scenario as Compared to Actual Data in 2013

\begin{tabular}{|c|c|c|c|c|c|c|c|}
\hline \multirow[b]{2}{*}{ Region } & \multicolumn{5}{|c|}{ Regulation Requirement } & \multicolumn{2}{|c|}{ Peak Load } \\
\hline & LowVG & RTx10 & RTx30 & ITx30 & 2013 & ERGIS 2026 & Actual $2013^{24}$ \\
\hline ISO-NE & $99-293$ & $122-315$ & $\begin{array}{l}187- \\
386\end{array}$ & $\begin{array}{l}169- \\
356\end{array}$ & $30-150$ & 29,208 & 27,400 \\
\hline MISO & $\begin{array}{c}634- \\
1,508\end{array}$ & $\begin{array}{l}800- \\
1,650\end{array}$ & $\begin{array}{c}875- \\
1,745\end{array}$ & $\begin{array}{l}1,074- \\
1,914\end{array}$ & $300-500$ & 148,894 & 98,576 \\
\hline NYISO & $134-352$ & $197-426$ & $\begin{array}{c}204- \\
453\end{array}$ & $\begin{array}{l}214- \\
454\end{array}$ & $150-250$ & 34,762 & 33,956 \\
\hline PJM & $\begin{array}{c}667- \\
1,881\end{array}$ & $\begin{array}{c}774- \\
1,928\end{array}$ & $\begin{array}{c}982- \\
2,137\end{array}$ & $\begin{array}{c}957- \\
2,075\end{array}$ & $\begin{array}{c}753 \\
\text { Average }\end{array}$ & 187,818 & 157,508 \\
\hline SPP & $202-521$ & $388-803$ & $\begin{array}{c}512- \\
1,157\end{array}$ & $\begin{array}{c}659- \\
1,520\end{array}$ & $\begin{array}{c}300 \\
\text { Average }\end{array}$ & 50,226 & 45,256 \\
\hline
\end{tabular}

In the LowVG and RTx10 scenarios, the Lew et al. (2013) reserve methodology procures more regulating reserves than is observed historically. This methodology results in the procurement of nearly twice as much regulation as has been observed in several regions. This may imply that the NREL assumption that the base regulation requirement of $1 \%$ of load is more conservative than the methodologies employed by the actual regions. This is most clearly apparent in NYISO and ISO-NE, where the 2013 peak load is very similar to the regional peak load in ERGIS. For example, the ERGIS regulation reserve requirements for the LowVG scenario in ISO-NE are both higher overall and have a wider range between than was actually procured by ISO-NE in 2013. In Table 36, we calculated the percent of peak load held in reserves for each scenario and compared it to 2013 data. This shows that the methods employed here result in nearly twice as much regulation reserve in the LowVG and RTx10 cases as the actual regions procured in 2013. In SPP, as the VG penetration increases, the amount of reserves increases considerably compared to the LowVG and 2013 data.

\footnotetext{
${ }^{24}$ Values derived from Denholm et al. (2015).
} 
Table 36. Regulation Reserves as a Percentage of Peak Load

\begin{tabular}{|l|l|l|l|l|l|}
\hline Region & $\begin{array}{l}\text { Actual } \\
\mathbf{2 0 1 3}\end{array}$ & LowVG & RTx10 & RTx30 & ITx30 \\
\hline ISO-NE & $0.5 \%$ & $1.0 \%$ & $1.1 \%$ & $1.3 \%$ & $1.2 \%$ \\
\hline MISO & $0.5 \%$ & $1.0 \%$ & $1.1 \%$ & $1.2 \%$ & $1.3 \%$ \\
\hline NYISO & $0.7 \%$ & $1.0 \%$ & $1.2 \%$ & $1.3 \%$ & $1.3 \%$ \\
\hline PJM & $0.5 \%$ & $1.0 \%$ & $1.0 \%$ & $1.1 \%$ & $1.1 \%$ \\
\hline SPP & $0.7 \%$ & $1.0 \%$ & $1.6 \%$ & $2.3 \%$ & $3.0 \%$ \\
\hline
\end{tabular}

These differences indicate that the ERGIS reserve requirements should be viewed, not as the optimal amount of regulation that regions "should" use, but rather as a consistently applied framework based on the requirements previously explained. Further analysis is needed to determine the impact of different reserve levels on reliability and operational cost. 


\section{UC\&ED Model Setup and Execution}

The UC\&ED model replicates both the day-ahead and real-time operational phases of the electric power sector. The 2026 EI model was simulated for an entire year in 8,760 one-hour time steps in the day-ahead phase of operations and 105,120 five-minute steps in the real-time phase of operations. This section describes the representation of transmission, limitations on interregional trade, and the configuration of model settings for day-ahead and real-time operations.

\subsection{Regional Coordination and Hurdle Rates}

We configured the UC\&ED model using hurdle rates to reflect elements of market friction between the ERGIS regions. Hurdle rates of $\$ 10 / \mathrm{MWh}$ were applied to any power transferred between the larger ERGIS regions shown in Figure 1, but not the smaller sub-regions shown in Figure 39. This means that in order to effectuate a trade of energy between regions, the spread between the costs had to be greater than $\$ 10 / \mathrm{MWh}$. As such, to trade from SPP to MISO, the cost in MISO would have to be \$10/MWh higher than SPP. Similarly, to wheel energy from SERC, through PJM, to NYISO would require a hurdle rate between each region, for a total cost of $\$ 20 / \mathrm{MWh}$.

Because bilateral contracts are confidential in nature, and likely to evolve considerably in the scenarios studied in ERGIS, all generators modeled in the study are co-optimized according to their assumed capabilities. This means the study does not represent any historical generation and associated transmission schedules in the model.

\subsection{Operational Assumptions for Wind and PV}

Wind and PV resources were assumed to have zero variable costs. Furthermore, the model did not include any production-tax credits. All wind and PV resources were assumed to be dispatchable up to their maximum available output. This means that the UC\&ED model could dispatch down wind and solar resources, otherwise known as VG curtailment, in order to balance system demands and respect a variety of other generation and transmission constraints. This assumption is practical for utility scale wind and PV projects, as most generators deployed in 2014 already have this capability. Both distributed and utility-scale PV are curtailable in our model, which reflects a greater degree of control of distributed PV than is currently present in most U.S. markets.

\subsection{Transmission Representation}

The UC\&ED model represents all of the transmission elements and uses DC power flow, which is a linearized approximation of $\mathrm{AC}$ power flow. The DC power flow approach decouples and neglects reactive power phenomena from real power flow. However, DC power flow is more representative of reality than the pipe-flow methods often used when simulating large systems. Enforcing all of the transmission constraints was infeasible because they caused the simulation times to be intractable. To keep simulation times reasonable, the number of transmission constraints was substantially reduced and transmission constraints were modeled as soft constraints with penalty prices for exceeding the flow limits.

To limit the number of transmission constraints in the model, the ERGIS team solicited and received feedback from the TRC on sub-regions within which transmission limits would not be 
enforced. Approximations of existing transmission or capacity zone boundaries were used in areas with restructured market regions (MISO, SPP, PJM, NYISO, and ISO-NE), and approximations of the service territories of the major operating companies were used in SERC. FRCC contained only one sub-region, as did each of the Canadian provinces. The ERGIS subregions are shown in Figure 39.

After establishing the sub-regions, transmission lines that crossed each of the sub-region boundaries were identified. These transmission lines were grouped by geographic proximity into flowgates. Figure 40 shows all of the lines that were included in a flowgate. The sum of the flows on the lines in the flowgate was then constrained rather than the flows on individual lines. This resulted in a significant reduction in the number of transmission constraints in the model. We limited inter-sub-regional transfers (between sub-regions) but not intra-sub-regional (within sub-regions) transfers. In order to reflect contingency limits, each flowgate was assigned a flow limit defined by the maximum of: half of the sum of the thermal ratings of the lines in the flowgate lines or the sum of the thermal ratings of the flowgate lines minus the largest thermal rating in the flowgate lines. Transfers along some transmission corridors, such as between FRCC and SERC, may be limited by voltage constraints or thermal constraints on facilities other than tie lines. For these areas, the flow limits assumed in this study will not reflect actual limits in the present-day system. Similarly, present-day intra-regional transmission line limits are not reflected in this study. 


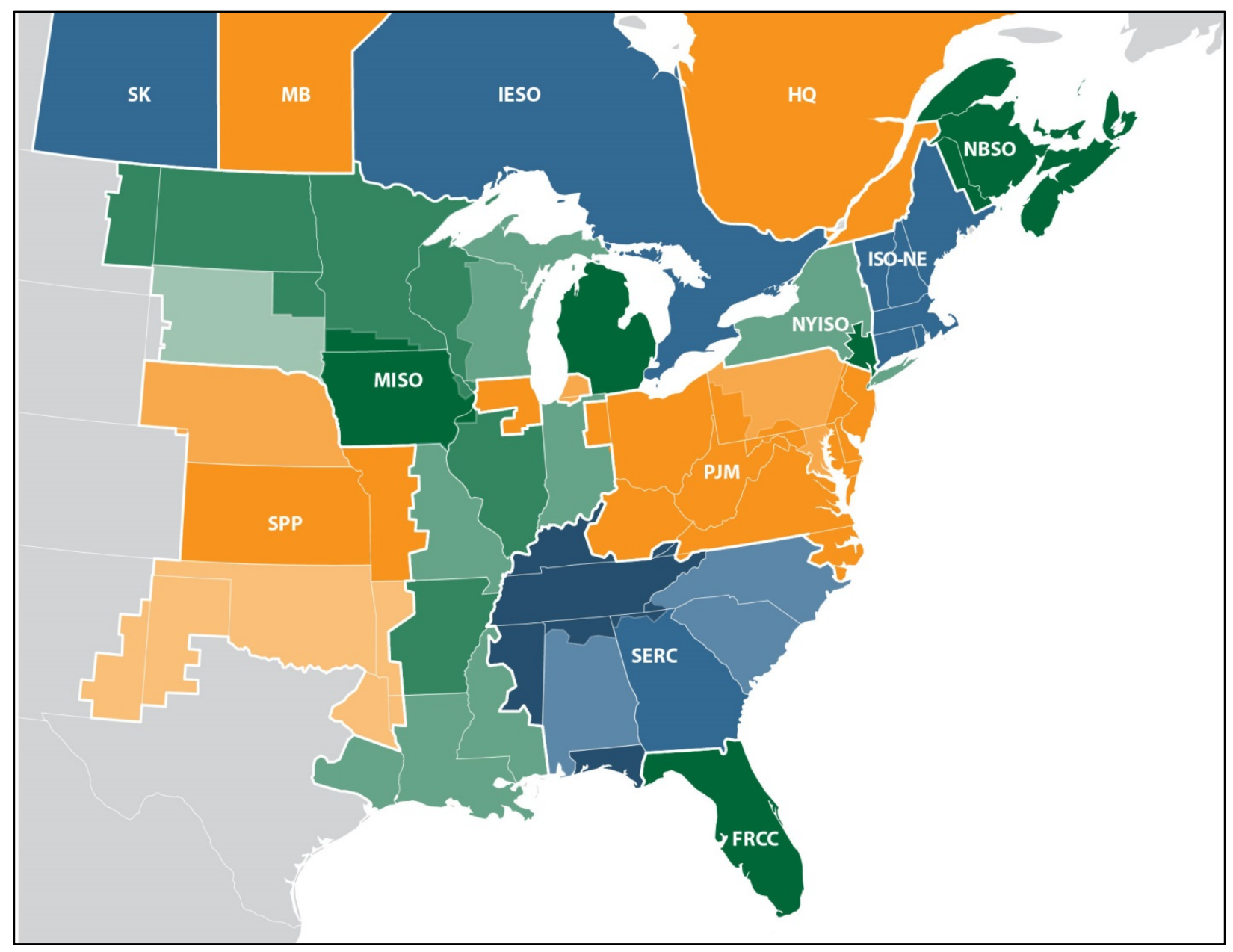

Figure 39. Map showing the ERGIS sub-regions, which were used as both transmission zones and load zones 


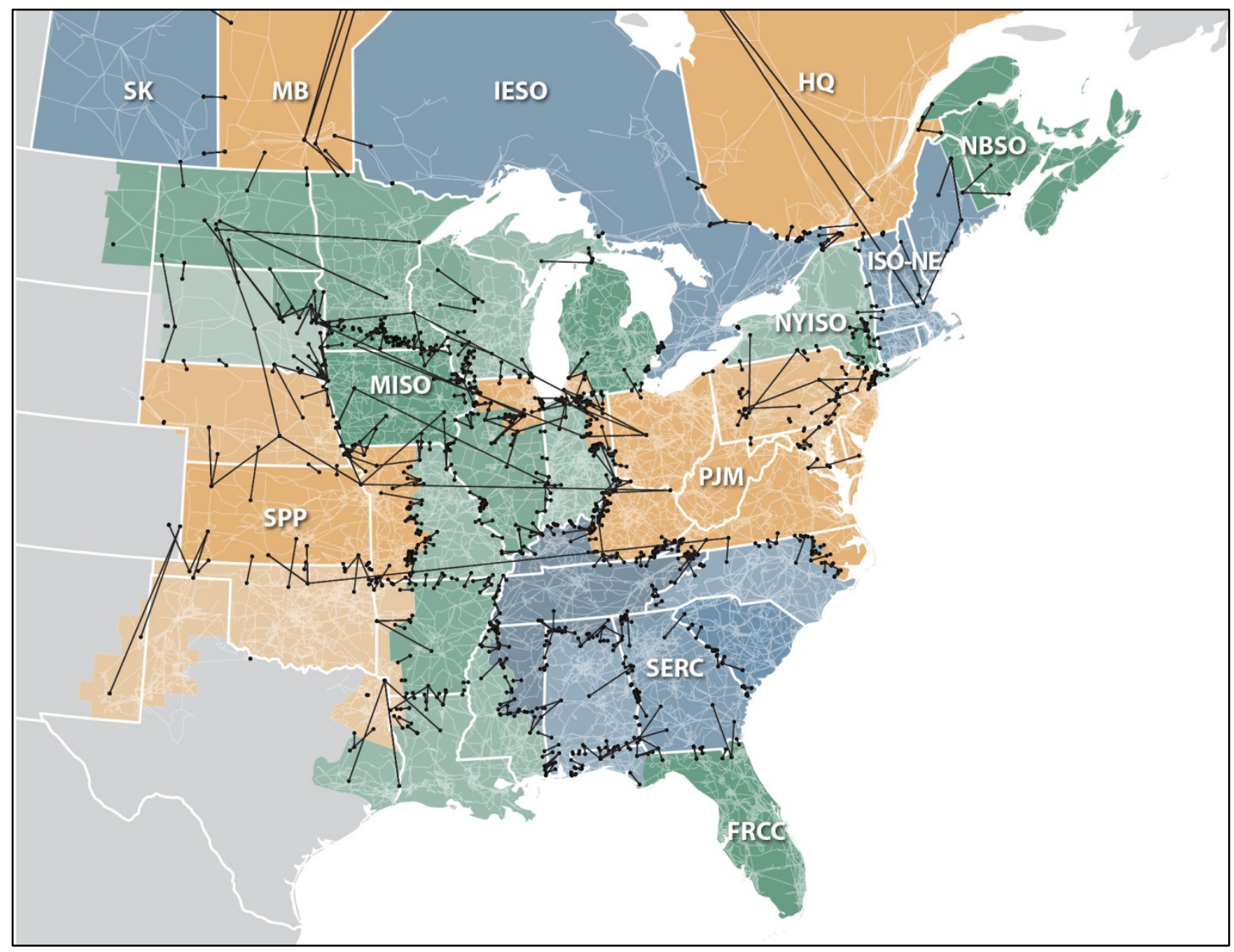

Figure 40. Map showing the transmission flowgates between the ERGIS sub-regions 


\subsection{Day-ahead Model Configuration}

The first stage of the UC\&ED model replicates the day-ahead phase of electricity market operations. In this stage the operator plans for the following day by scheduling the commitment (on or off status) of most generators for each hour of the following day. In ERGIS, this stage was assumed to occur about mid-day for operations that occur the following day. The day-ahead modeled a 1-hour resolution for the next day plus an additional day of look-ahead at 6-hour resolution. After each day of the day-ahead phase is solved, the next day is solved with a new day of look-ahead, until the full year of operation is completed.

The day-ahead uses forecasted generation data for wind and PV generation but assumes perfect knowledge of the load (i.e. perfect load forecast). The day-ahead forecasts for wind and PV are described in Chapter 4. The day-ahead forecasts represent a forecast generated at mid-day for generation from midnight to midnight of the following day, in order to match the operational sequence of the major restructured market regions.

The day-ahead procures capacity for the regulation and contingency reserves described in Chapter 3. The reserves requirements are soft constraints with penalty prices to allow for reserve shortages.

\subsection{Real-time Model Configuration}

The second stage of the UC\&ED model replicates the real-time phase of electricity market operations. In this stage the system operator dispatches the output of online generators to meet the load and adjust for forecast errors in wind and PV generation. The real-time model considers a single 5-minute interval without look-ahead, solves for the optimal operation, and then moves forward to the next 5-minute interval and repeats until the whole year is completed.

The real-time uses several pieces of information from the day-ahead phase. In the real-time, the generator commitment schedules for coal, biomass, combined cycle natural gas, and boiler units were fixed from the day-ahead. Because the real-time does not have any look-ahead, the model conservatively assumed that all hydro and pumped hydro energy storage facilities had real-time generation schedules that were held constant from the day-ahead simulation. The real-time uses the synthetic "actual" 5-minute wind and PV data which is different than the forecast used in the day-ahead.

The real-time simulation is assumed to run a few minutes before the actual time it represents, such that generators with short startup times (combustion turbines and internal combustion engines) can be started in the real-time.

The real-time also re-optimizes the capacity reserved for the regulation and contingency reserves described in Chapter 6. No actual deployment of these reserves is modeled, except that the solver will choose to violate a soft reserve constraint rather than any of the hard constraints such as energy balance, generator operational constraints, etc.

\subsection{Model Execution}

The model was executed using PLEXOS version 6.400 R02. The PLEXOS model for ERGIS includes over 7,500 generating units, 60,000 transmission nodes, and 70,000 transmission lines 
and transformers. As formulated by PLEXOS, each day-ahead optimization problem has about 73,000 integer variables, 1.2 million continuous variables, and 23 million non-zeros in the constraint matrix. Because of the size of the problem, runtimes would be infeasible if the model were run consecutively from January 1 through December 31 of the study year.

To address the extreme computational challenge presented by large UC\&ED models, previous work at NREL developed methods to decompose annual UC\&ED simulations into shorter time partitions while preserving the accuracy of simulation results (Barrows et al. 2014). The effort resulted in a method for time domain decomposition and parallel simulation of production cost models. Figure 41 illustrates how an annual simulation might be decomposed into 4 partitioned simulations. Inter-temporal constraints are required to represent the time that it takes to turn on/off a generator and to change the output of a generator. While the accuracy of UC\&ED simulations is dependent, in part, on the enforcement of these inter-temporal constraints, the effects of enforcement in earlier simulation steps diminish. For example, while a certain generator may have a binding minimum on time sometime during January, that constraint is unlikely to affect that same generator's operation in August. Additionally, the inter-temporal constraints require the sequential simulation of each step in the horizon, which can lead to extremely long computation times.

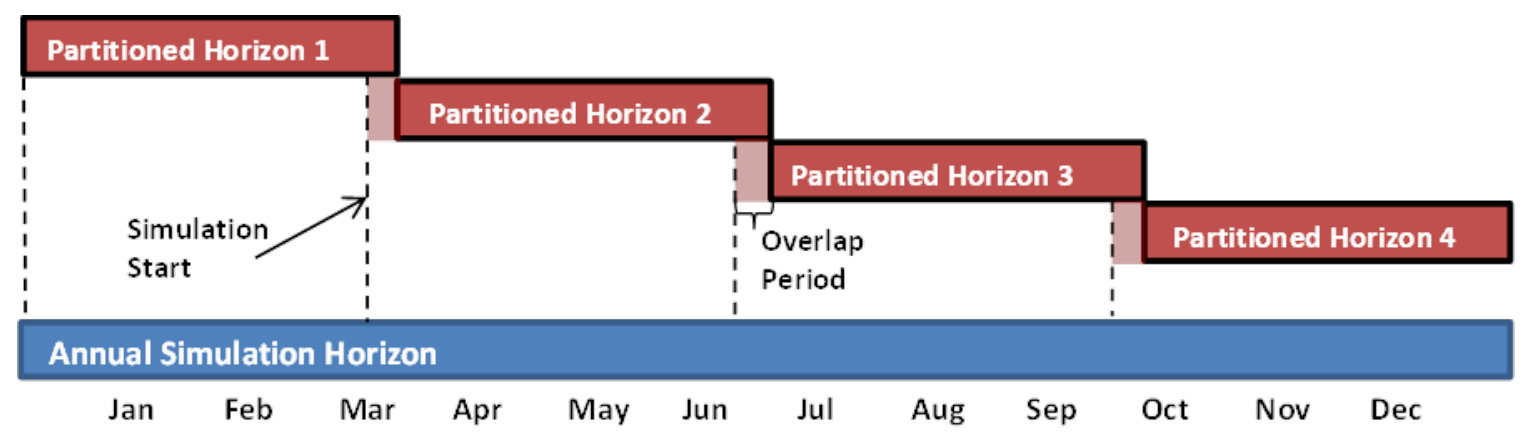

Figure 41. Illustration of annual (blue) vs. decomposed (red) UC\&ED horizons

The overlap period shown in Figure 41 at the beginning of partitioned horizons 2-4 is a set of extra simulation periods that enable more accurate simulation of the partitioned model. For example, if partitioned simulation starts on midnight of April 1 without information about system operation in March, no information about how long generators have/have not been operating is passed to the start conditions of the simulation period, ${ }^{25}$ and thus the simulation cannot represent the inter-temporal constraints that define generator operation. Adding overlap periods to the beginning of the simulation step gives the simulation time to establish operational history so that inter-temporal constraints can be enforced throughout the duration of the partitioned horizon. The amount of overlap required to minimize the error introduced by partitioning is roughly 2 simulation periods (days) (Barrows et al. 2014).

ERGIS used the time domain partitioning method to parallelize PLEXOS UC\&ED simulations using NRELs Peregrine high performance computing (HPC) system (NREL 2015). The massively parallel architecture of the HPC system enabled simultaneous execution of annual

\footnotetext{
${ }^{25}$ By default, PLEXOS considers all inter-temporal constraints non-binding at the beginning of simulation horizons.
} 
UC\&ED simulations partitioned into 73 independent simulation horizons. After partitioning, each day-ahead simulation horizon consisted of 5 simulation steps (days) with an additional 2 overlap steps. Figure 42 shows the computational tractability improvements achieved through partitioning and parallel simulation methods. The approximately 30x speedup enables annual simulations that were projected to take in excess of 545 days to be completed in roughly 19 days.

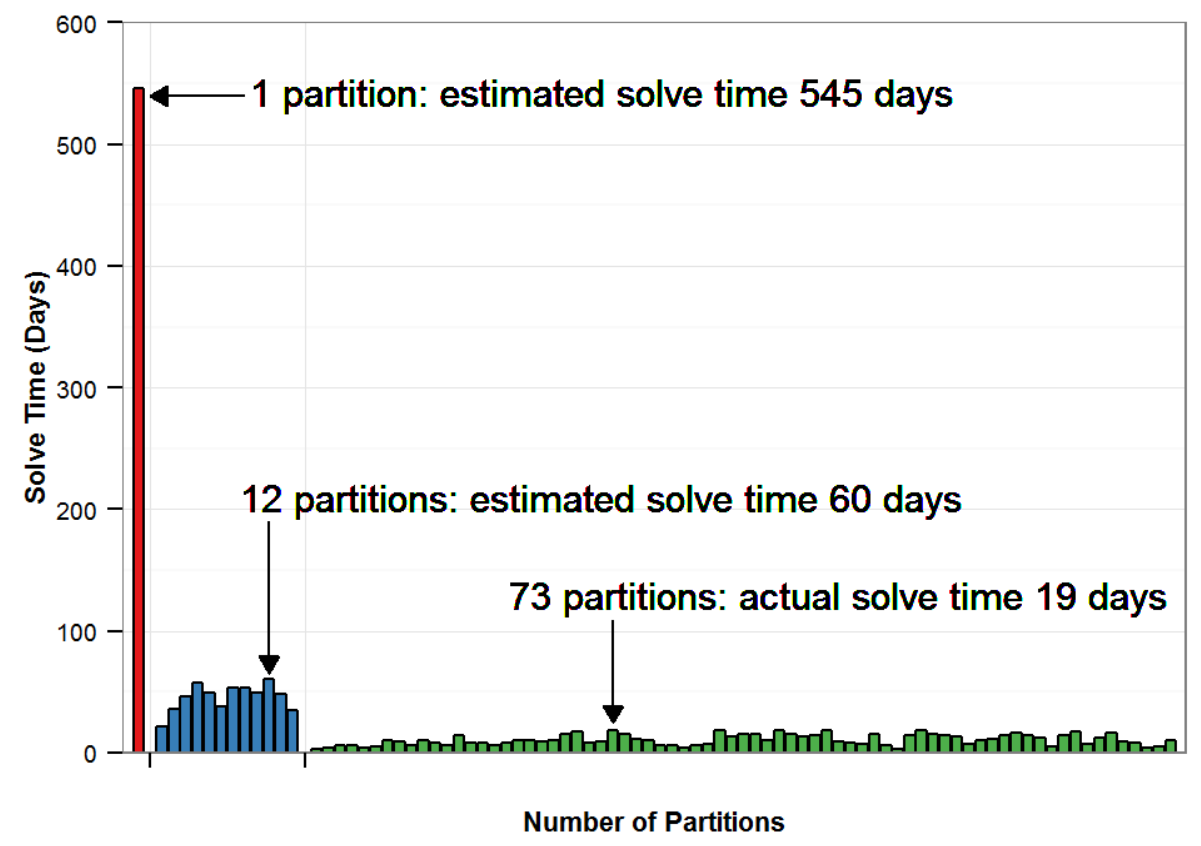

Figure 42. Computation times for annual simulations before and after partitioning and parallel computation methods were applied. 


\section{Operational Impacts and Modeling Results}

We analyzed the results of the unit commitment and economic dispatch modeling conducted with PLEXOS using a variety of operational metrics (e.g. annual generation, ramping, capacity factor, VG curtailment) in order to understand how varying levels of wind and PV generation affect system operations. The metrics were then compared across scenarios at both an interconnection-wide and regional level.

\subsection{Annual Generation}

Wind and PV resources often displace other sources of generation because of their low (or zero) variable costs and that was generally the case in this analysis of annual generation. Wind and PV had equivalent impact on traditional generation in the RTx30 and ITx30 at an interconnection wide level. However, regional analysis indicates that the differences between the RTx30 and ITx30 scenarios can result in significant regional impacts. For example, concentration of wind in MISO in the ITx30 results in significantly more displacement of Coal, Gas CC, and CT/Gas boilers than the RTx30. Additionally, the higher penetration of solar in the RTx30, coupled with increased reserve requirements, result in higher utilization of CT/Gas boilers, as compared to the ITx30.

Figure 43 shows the total generation for the U.S. EI, and Figure 44 shows generation differences between the LowVG and the rest of the scenarios. At the U.S. EI-wide level, generation levels from coal, CC, and CT/Gas boiler in the RTx30 and ITx30 are roughly equal. This indicates that the difference in the VG and transmission assumptions between the RTx30 and ITx30 scenarios have nearly identical impacts to generation, at interconnection wide level.

Coal, CC, and CT/Gas boiler generation are displaced as the amount of VG increases across the scenarios. Nuclear generation was assumed to be inflexible and therefore does not change across scenarios. In the LowVG case, coal makes up $46 \%$ of all generation, which decreases to $34 \%$ in the RTx30 and $33 \%$ in the ITx 30 . CC generation also changes substantially, with a decrease in generation from $22 \%$ in the LowVG to just $12 \%$ in the RTx30 and ITx 30 . CT/Gas boiler generation in the ITx30 and RTx30 is half as much as in the LowVG and RTx10 scenarios. 


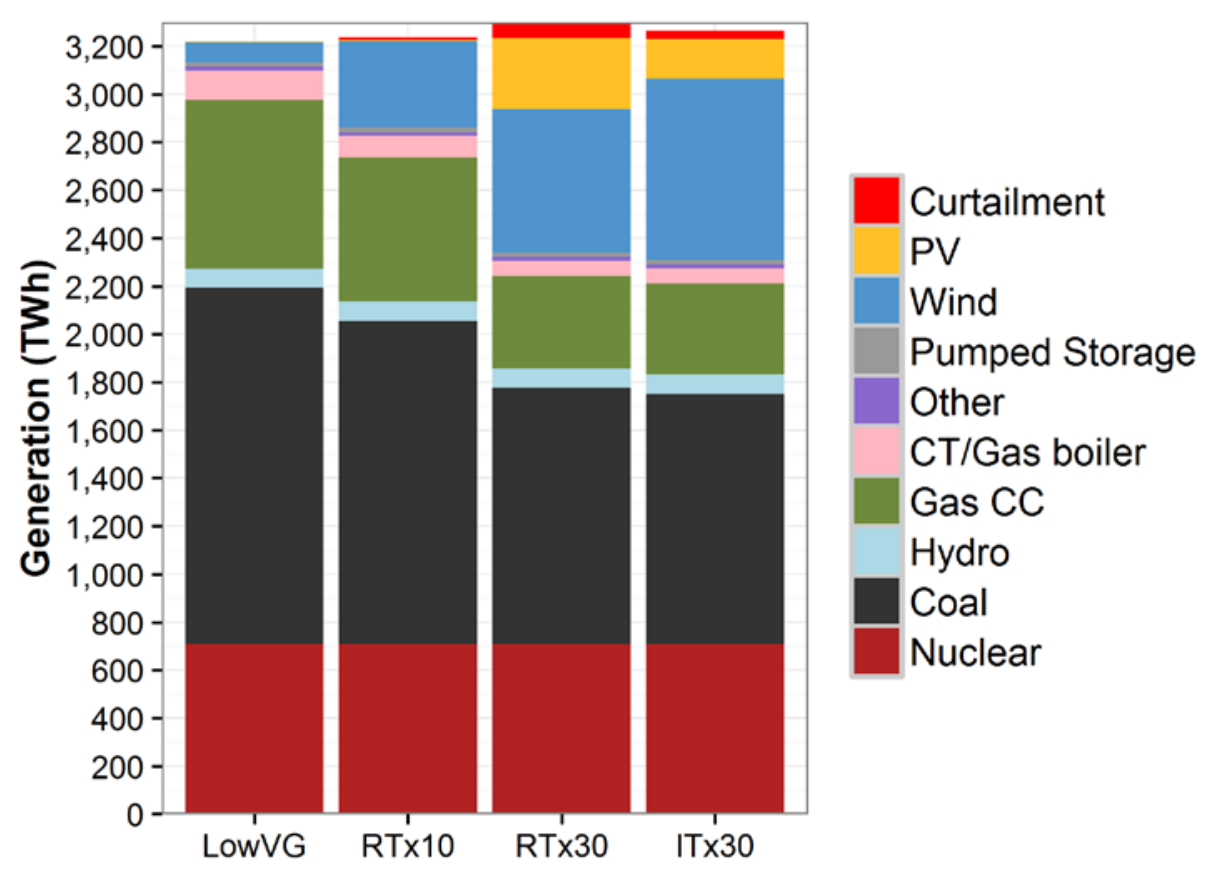

Figure 43. Annual generation by generator type for the U.S. Eastern Interconnection across all four scenarios

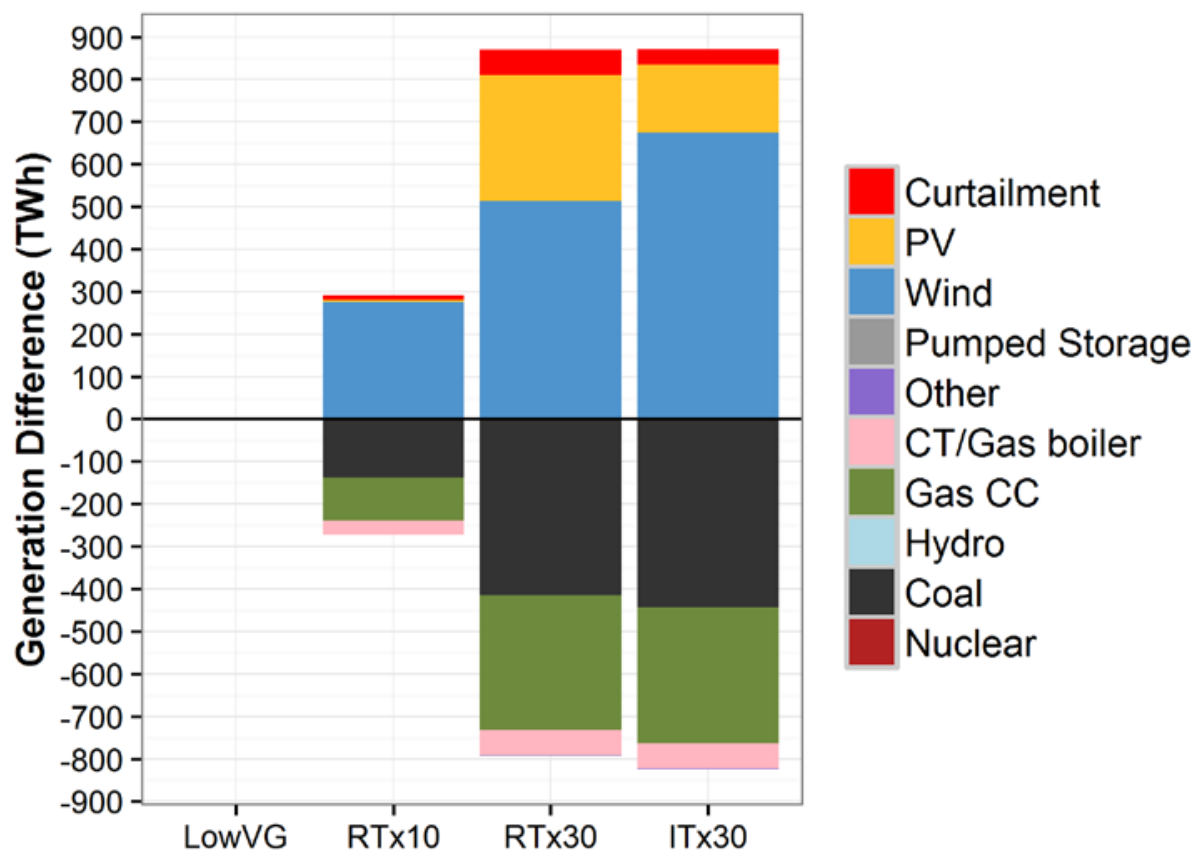

Figure 44. Differences between LowVG and other scenarios in annual generation by generator type for the U.S. EI 
Figure 45 shows the annual average of the instantaneous VG penetration and the maximum instantaneous (5-minute) penetration, as a percentage of the total generation. Because the penetration levels evaluated in this study are based on total annual energy, VG penetration exceeds the $10 \%$ and $30 \%$ annual penetrations during numerous periods. These high periods are balanced by other periods that have lower VG penetration. The annual penetration for the LowVG, RTx10, RTx30, and ITx30 were 3\%, 11\%, 28\%, and 29\%, respectively.

Despite the modest annual penetration of wind and PV in the RTx10 for the U.S. EI, the system experiences some very high regional instantaneous penetrations, as a percentage of total generation, most notably SPP (85\%), NYISO (54\%), MISO (40\%) and ISO-NE (37\%). Even with very little VG installation in the southeastern United States, the U.S. EI reaches an instantaneous penetration of $25 \%$ in the RTx 10 .

In the RTx30 and ITx30, with higher VG penetration levels, the highest instantaneous penetration for the U.S. EI occurs in the RTx30. There, VG penetration peaks at $60 \%$ of all generation. The RTx30 also has the highest instantaneous penetration level for most of the regions. This is because capacity in that scenario is more dispersed across the interconnection. The regional exceptions to this trend are MISO and SPP, which have their highest penetration in the ITx30. The maximum instantaneous penetration of VG in MISO is $74 \%$ in the ITx30, compared to $62 \%$ in the RTx 30 . The difference in maximum penetration for SPP only differs slightly between the ITx30 and RTx30. The instantaneous penetration in the ITx30 is 94\%; in the RTx30 that value decreases to 93\%. Under both 30\% scenarios, SPP averages more than 50\% penetration of $\mathrm{VG}$, with periods where less than $10 \%$ of energy is from synchronous generation.

The largest disparities between average and maximum penetrations occur in SERC and FRCC, the regions with the highest fractions of PV generation. This is not surprising as PV only generates during the day. This results in very high instantaneous penetrations of $84 \%$ in the RTX30 for FRCC, but lower penetrations on average, compared to other regions. 


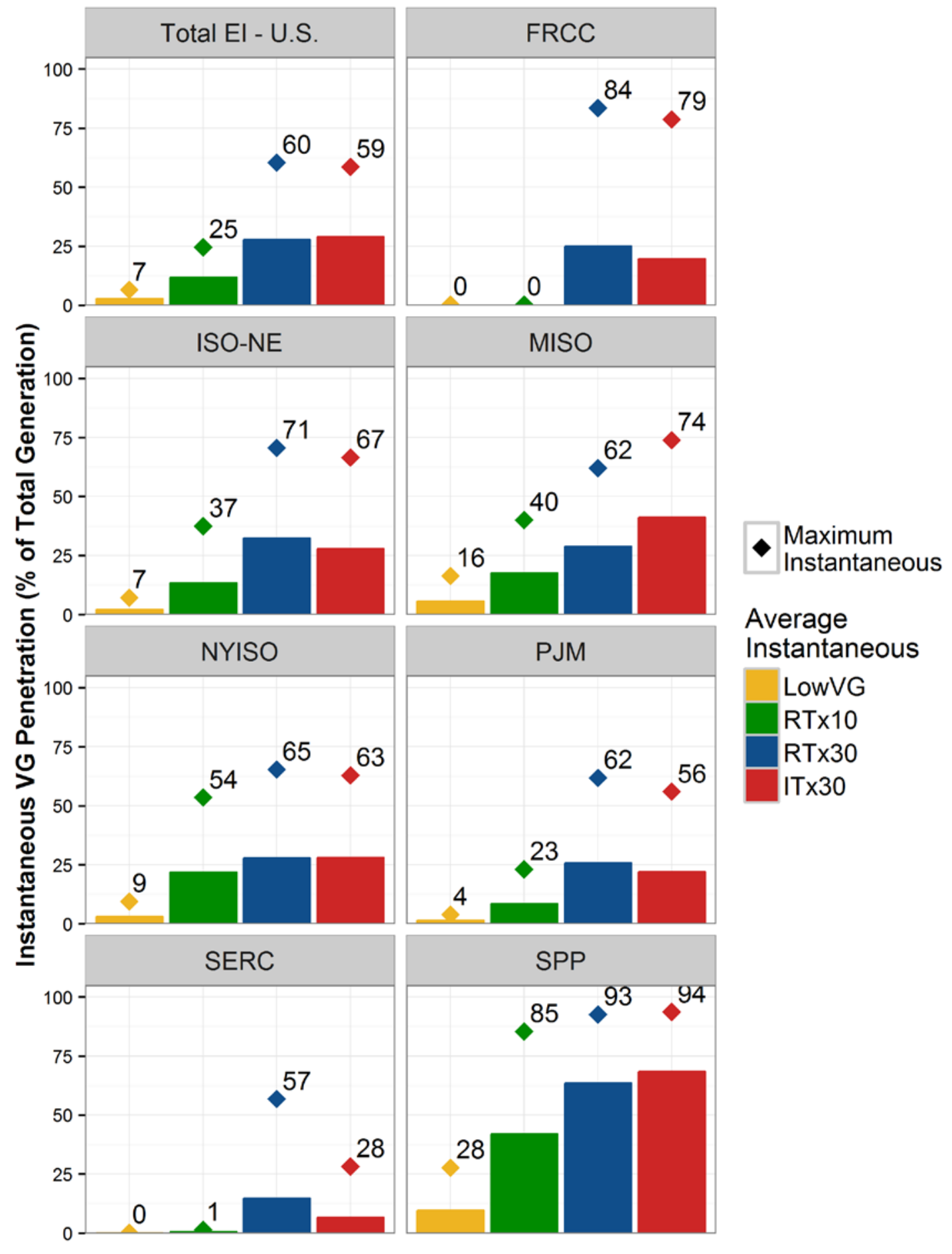

Figure 45. Instantaneous penetration of VG as a percent of total generation

VG penetration duration curves for each scenario are shown in Figure 46. The RTx30 and ITx30 curves between the $30 \%$ cases are very similar. The RTx30 has some periods of higher penetration and is slightly steeper than the ITx30, indicating greater extremes in the RTx30 and more consistent VG penetration in the ITx30 for the U.S. EI as a whole. This difference in slope is largely due to the differences in PV capacity. There is twice as much PV capacity in the 
RTx30 as in the ITx30. With more PV there are larger disparities between peaks of PV output in the middle of the day and troughs of PV output at night.

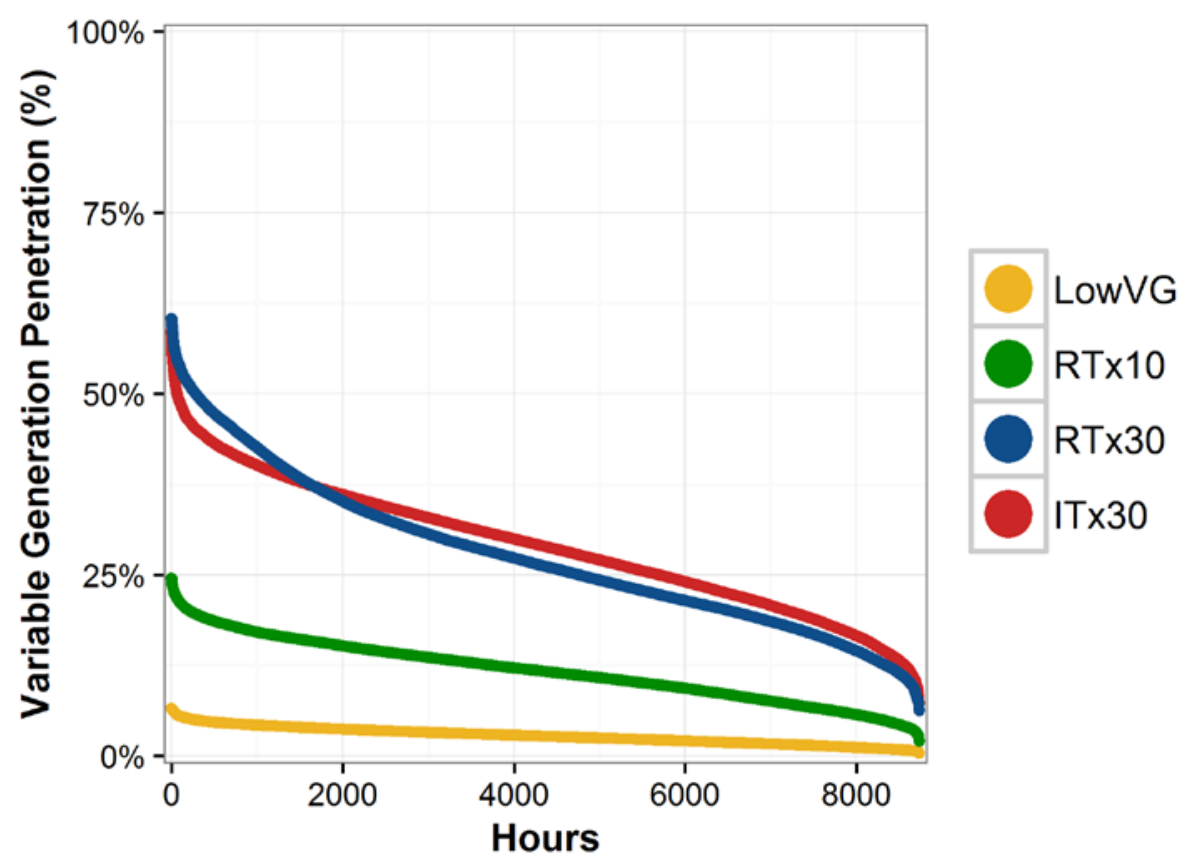

Figure 46. Duration curve of VG penetration for all scenarios for the U.S. EI

Table 37 shows the fuel usage (in quads, or quadrillion BTU), consumed by coal and gas generators in each of the scenarios. In the LowVG, generators used 6.1 quads of gas, and 15.3 quads of coal. As VG penetration increases, fuel use declines. In the two $30 \%$ scenarios, the U.S. EI uses about one third less coal and about half as much gas.

Table 37. Fuel Usage by Coal and Gas Generators in ERGIS Scenarios (quadrillion BTUs)

\begin{tabular}{|l|r|r|r|r|} 
& \multicolumn{1}{|c|}{ LowVG } & \multicolumn{1}{|c|}{ RTx10 } & \multicolumn{1}{|c|}{ RTx30 } & \multicolumn{1}{|c|}{ ITx30 } \\
\hline Coal & 15.3 & 13.9 & 11.0 & 10.8 \\
\hline Gas & 6.1 & 5.0 & 3.3 & 3.3 \\
\hline
\end{tabular}

Differences between annual thermal and hydro plant generation in the ITx30 and RTx30 were minimal on an interconnection-wide basis, but regional differences were sometimes substantial. Figure 47 shows the annual generation for all scenarios for each U.S. region, as well as the load. Although the modeled scenarios change the installed VG resources, net exports between regions do not change significantly between scenarios. The ITx30, due to the added transmission capacity and concentration of VG in the western portion of the system, results in more exports from SPP and more imports in PJM, MISO, and SERC. 


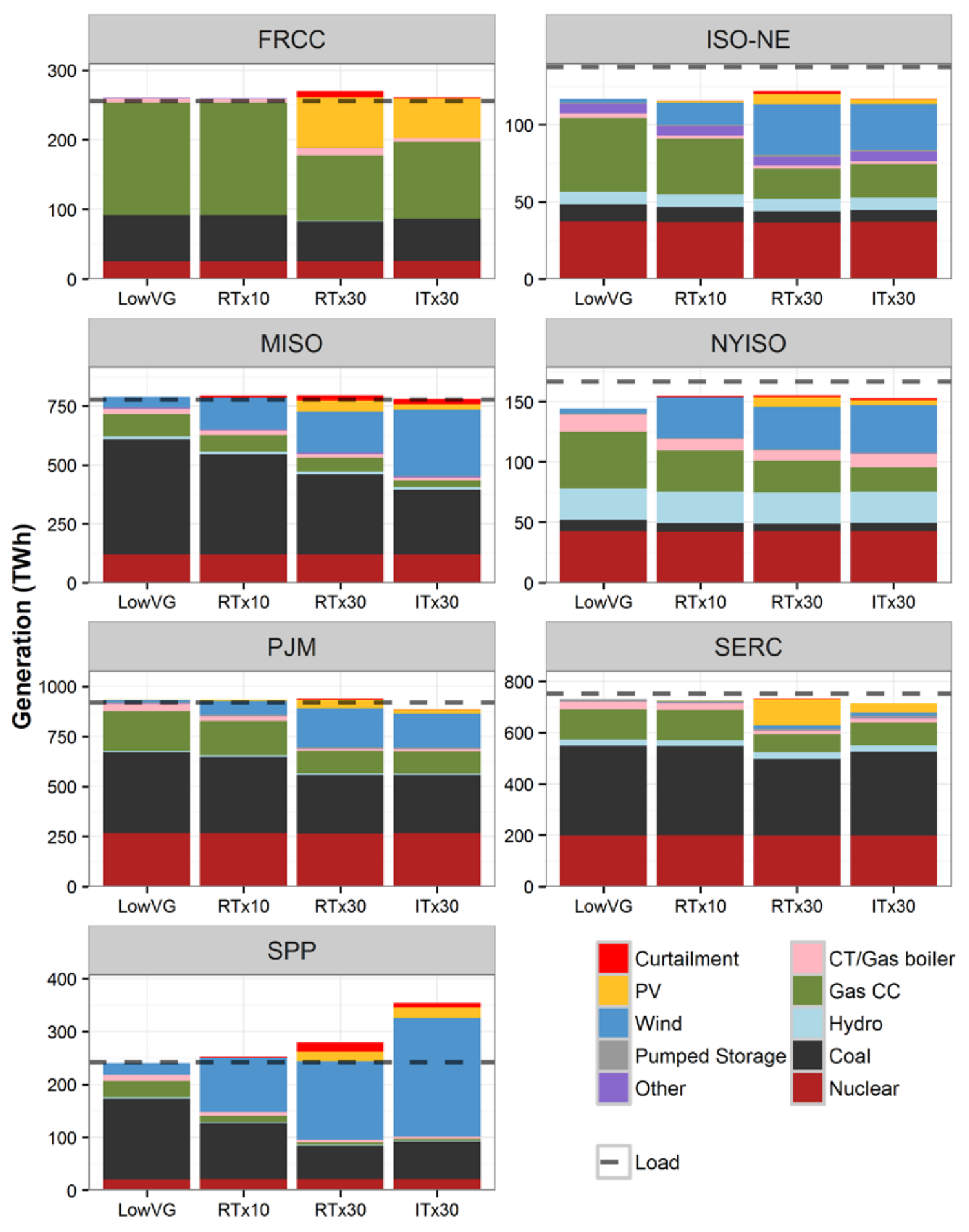

Figure 47. Annual load and generation by generator type for all U.S. El regions (note the changing scales on the y-axis)

Using LowVG generation as a baseline or zero point, Figure 48 highlights the differences in generation in each scenario and U.S. EI region. The thermal plant displacement in MISO is highest in the ITx30 because in the ITx30 new wind capacity is installed predominantly in 
MISO. Similarly, SERC experiences more regional displacement in the RTx30 because the RTx30 requires intraregional VG resources to be deployed in SERC, rather than imported from another region. The impact of new wind and PV generation on thermal generators varies across regions and transmission scenarios.

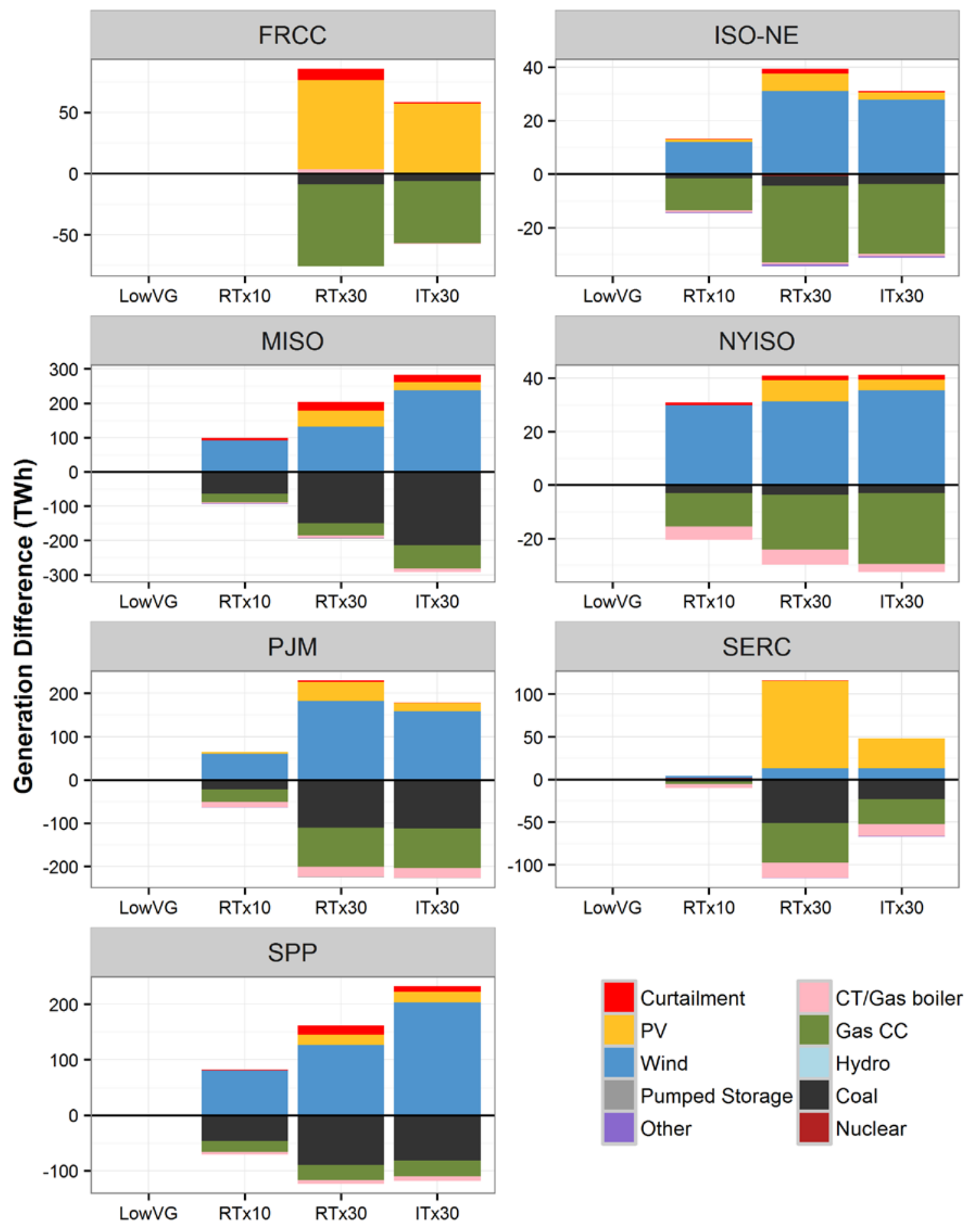

Figure 48. Difference in annual generation between LowVG and other scenarios, by generator type (note the changing scales on the $y$-axis) 
Table 38 through Table 44 detail the percentage difference in fossil-fueled generation for each regioncompared to the LowVG. In the LowVG, CT/Gas boilers generate during peak load hours and their output generally decreases as VG is added to the system. These tables highlight regional differences in system response to the addition of VG. For example:

- In FRCC, there is a noticeable increase in $\mathrm{CT} / \mathrm{Gas}$ boiler generation as the penetration of $\mathrm{PV}$ increases. This change is unique to $\mathrm{FRCC}$, as $\mathrm{CT} / \mathrm{Gas}$ boiler operation decreases in every other study region for the RTx30 and ITx30. This is a result of the decreased commitment of CCs during the daylight hours with higher PV generation, resulting in $\mathrm{CT} /$ Gas boilers being utilized more for short duration peaking events when solar is going offline at the end of the day. While significant with respect to $\mathrm{CT} / \mathrm{Gas}$ boiler generation, from the perspective of total generation the increase is small, as indicated in Figure 48. FRCC also experiences significant displacement of CC in the $30 \%$ scenarios.

- ISO-NE experiences significant displacement of CC generation as the wind, PV, and transmission systems change across the scenarios. As in FRCC and SERC, the ISO-NE thermal units experience the largest displacement in the RTx30.

- In MISO, differences in the operational impacts of VG deployment strategies may not be apparent when analyzed at an interconnection-wide level. Several interconnection-wide metrics have nearly identical values across scenarios, but in MISO, the largest geographic region in the U.S. EI, those values still vary significantly between the scenarios. As seen in Figure 48, MISO experiences the largest thermal generation displacement in the U.S. EI. Over $70 \%$ of all Gas CC generation is displaced in the ITx30, while Gas CC displacement in the RTx30 is $38 \%$.

- In NYISO, thermal plant displacement is generally greater in the RTx30 than the ITx30. Coal and CT/Gas boiler generation is lowest in the RTx30, while CC displacement is higher in the ITx30. Unlike other regions, the VG penetration level for NYISO does not change appreciably between the ITx30 and RTx30, however the generation mix does. Total VG penetrations for NYISO in those scenarios are within one half of one percent of one another.

- PJM thermal plant displacement increases considerably between the RTx10 and the 30\% scenarios. However, differences in displacement between the ITx30 and RTx30 are small. Coal and CC generation are virtually identical in both 30\% scenarios. PJM experiences the most CT/Gas boiler displacement in the study, on both an absolute and percentage basis. CT/Gas boiler generation decreases $70 \%$ in the RTx30 and $66 \%$ in the ITx 30 . The similarities in thermal plant displacement exist under both scenarios because local wind generation is replaced by remote wind generation imported from MISO and SPP via the larger transmission expansion in ITx30, including the highly-utilized HVDC lines. The utilization of the HVDC lines is described in Section 8.2.3.

- In SERC, because very small amounts of VG are deployed in the RTx10, only minor displacement of thermal plants occurs. This displacement is driven by VG capacity to meet state RPS in neighboring regions, therefore leading to more imports. Thermal plant displacement is highest in the RTx30. 
- SPP has the highest regional VG penetration levels in the study. This results in significant amounts of thermal plant displacement. Notably, in the ITx30, CC generation is almost completely displaced from the market by VG.

Table 38. Difference in Coal- and Gas-fueled Generation from LowVG for FRCC (TWh)

\begin{tabular}{|l|r|r|r|}
\hline Scenario & \multicolumn{1}{|c|}{ Coal } & \multicolumn{1}{|c|}{ Gas CC } & CT/Gas boiler \\
\hline LowVG & - & - & - \\
\hline RTx10 & $0(0 \%)$ & $0(0 \%)$ & $0(-6 \%)$ \\
\hline RTx30 & $-9(-14 \%)$ & $-67(-41 \%)$ & $4(55 \%)$ \\
\hline ITx30 & $-6(-9 \%)$ & $-51(-32 \%)$ & $-1(-9 \%)$ \\
\hline
\end{tabular}

Table 39. Difference in Coal- and Gas-fueled Generation from LowVG for ISO-NE (TWh)

\begin{tabular}{|l|r|r|r|}
\hline Scenario & Coal & Gas CC & CT/Gas boiler \\
\hline LowVG & - & - & - \\
\hline RTx10 & $-1(-11 \%)$ & $-12(-25 \%)$ & $-1(-27 \%)$ \\
\hline RTx30 & $-4(-33 \%)$ & $-29(-59 \%)$ & $-1(-28 \%)$ \\
\hline ITx30 & $-3(-31 \%)$ & $-26(-54 \%)$ & $-1(-35 \%)$ \\
\hline
\end{tabular}

Table 40. Difference in Coal- and Gas-fueled Generation from LowVG for MISO (TWh)

\begin{tabular}{|l|r|r|r|}
\hline Scenario & \multicolumn{1}{|c|}{ Coal } & Gas CC & CT/Gas boiler \\
\hline LowVG & - & - & - \\
\hline RTx10 & $-64(-13 \%)$ & $-25(-26 \%)$ & $-4(-19 \%)$ \\
\hline RTx30 & $-149(-$ & $-36(-38 \%)$ & $-8(-35 \%)$ \\
\hline ITx30 & $30 \%)$ & & $-10(-45 \%)$ \\
\hline
\end{tabular}

Table 41. Difference in Coal- and Gas-fueled Generation from LowVG for NYISO (TWh)

\begin{tabular}{|l|r|r|r|}
\hline Scenario & Coal & Gas CC & CT/Gas boiler \\
\hline LowVG & - & - & - \\
\hline RTx10 & $-3(-27 \%)$ & $-12(-27 \%)$ & $-5(-35 \%)$ \\
\hline RTx30 & $-4(-36 \%)$ & $-21(-44 \%)$ & $-6(-39 \%)$ \\
\hline ITx30 & $-3(-30 \%)$ & $-26(-57 \%)$ & $-3(-22 \%)$ \\
\hline
\end{tabular}


Table 42. Difference in Coal- and Gas-fueled Generation from LowVG for PJM (TWh)

\begin{tabular}{|l|r|r|r|}
\hline Scenario & \multicolumn{1}{|c|}{ Coal } & Gas CC & CT/Gas boiler \\
\hline LowVG & - & - & - \\
\hline RTx10 & $-21(-5 \%)$ & $-29(-14 \%)$ & $-13(-38 \%)$ \\
\hline RTx30 & $--109(-$ & $-90(-45 \%)$ & $-24(-70 \%)$ \\
\hline ITx30 & $-111(-27 \%)$ & $-92(-46 \%)$ & $-22(-66 \%)$ \\
\hline
\end{tabular}

Table 43. Difference in Coal- and Gas-fueled Generation from LowVG for SERC

\begin{tabular}{|l|r|r|r|}
\hline Scenario & \multicolumn{1}{|c|}{ Coal } & \multicolumn{1}{|c|}{ Gas CC } & CT/Gas boiler \\
\hline LowVG & - & - & - \\
\hline RTx10 & $-3(-1 \%)$ & $-3(-2 \%)$ & $-5(-16 \%)$ \\
\hline RTx30 & $-51(-15 \%)$ & $-46(-39 \%)$ & $-18(-59 \%)$ \\
\hline ITx30 & $-23(-7 \%)$ & $-30(-25 \%)$ & $-14(-47 \%)$ \\
\hline
\end{tabular}

Table 44. Difference in Coal- and Gas-fueled Generation from LowVG for SPP

\begin{tabular}{|l|r|r|r|}
\hline Scenario & Coal & Gas CC & \multicolumn{1}{|c|}{ CT/Gas boiler } \\
\hline LowVG & - & - & - \\
\hline RTx10 & $-46(-30 \%)$ & $-20(-64 \%)$ & $-4(-38 \%)$ \\
\hline RTx30 & $-90(-59 \%)$ & $-27(-87 \%)$ & $-7(-59 \%)$ \\
\hline
\end{tabular}

\subsection{Transmission Utilization}

As described in Section 4.3.5, ERGIS adopted the EIPC transmission expansions, which include planned transmission builds as well as hypothetical transmission expansions to meet the simulated increase of wind capacity in the SPP and MISO in the three EIPC scenarios.

The two 30\% cases highlight the role transmission plays in determining how VG can be used around the EI. The increased transmission capacity in the ITx30 allows greater trade between regions and increased utilization of the resources in MISO and SPP. The ITx30 case has about $55 \%$ more interchange than the RTx30 case. And, as illustrated in the case of SERC and PJM, greater local resources in RTx30 lead to more local generation and less need for imports than in ITx30.

\subsubsection{Energy Interchange Between Regions}

Figure 49 shows the total annual energy interchanged between the regions in the four scenarios. ${ }^{26}$ Trends in interchange between regions do not change substantially across scenarios. Regions that import energy in the LowVG continue to import energy in the RTx30 and ITx30 scenarios. The exception to this trend is found in SPP, which imports in the LowVG but exports in the RTx10, RTx30 and ITx30. While the annual trends are steady, closer inspection reveals

\footnotetext{
${ }^{26}$ Appendix $\mathrm{F}$ has a table of the magnitudes that make up Figure 50.
} 
important differences in the scale of interchange between the scenarios, and the direction of interchange between neighboring regions. For example, the pattern of flow between MISO, Manitoba, and IESO differs in every scenario. These changes in pattern are influenced by the interchange between MISO and its other neighbors; PJM, and SERC increase their imports from MISO as VG penetration increases. At the same time, PV in the Southeast influences the imports to SERC, especially in the RTx30.

The largest changes in net interchange are observed between regions that have significant increases in transfer capability and expanded wind and PV generation (see Figure 18-21), such as interchanges between SPP and MISO and SPP and PJM. For example, the ITx30 scenario assumes the addition of two HVDC lines between SPP and PJM, and these lines facilitate a net annual interchange of $36 \mathrm{TWh}$ between two regions that do not otherwise share a direct electrical interconnection. The ITx30 scenario also assumes new HVDC transmission capacity between the MISO-PJM-SPP systems, but the impact of these ties is not limited to these regions.

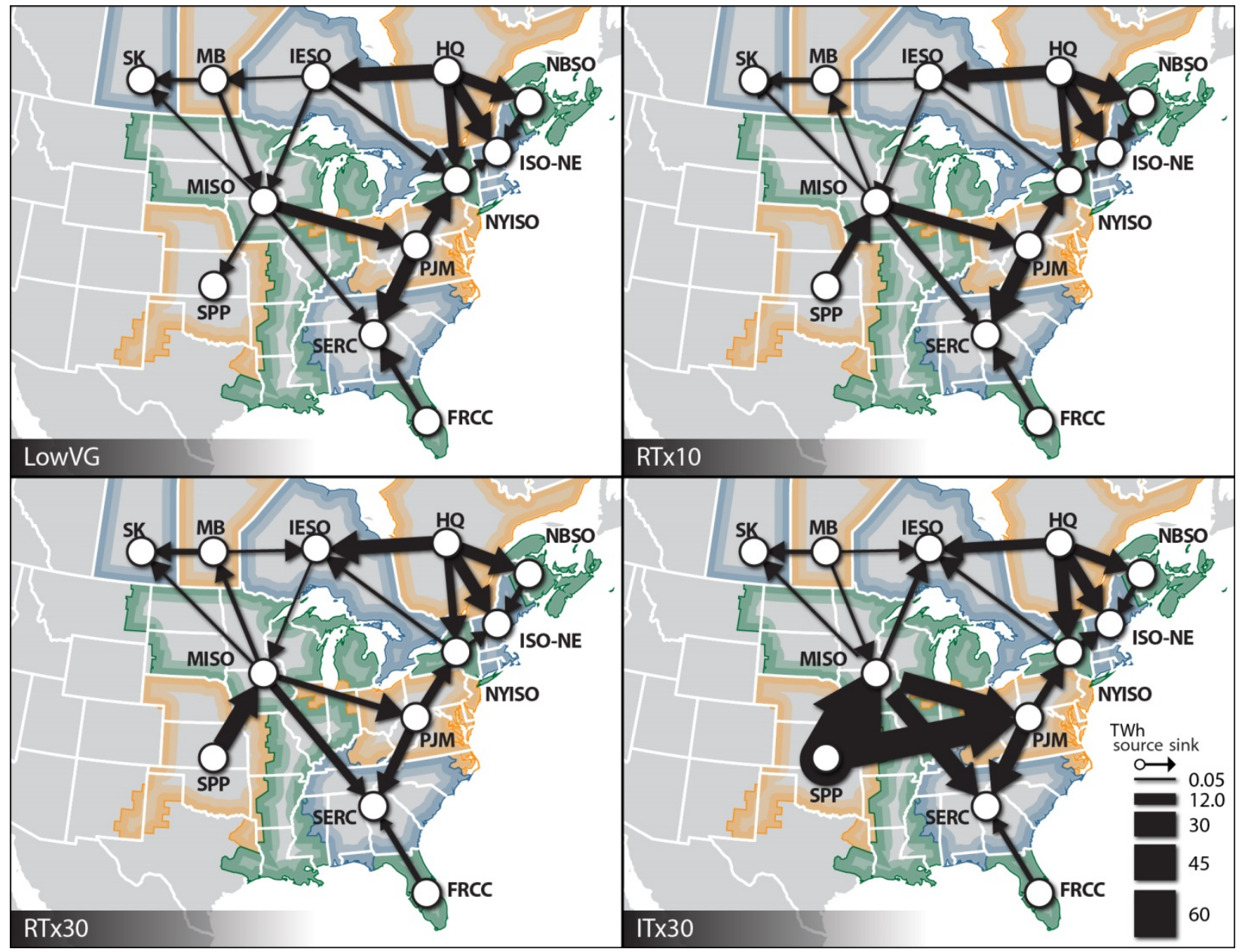

Figure 49. Total net interchange between regions

Table 45 shows the U.S. EI's net imports from Canada in each of the scenarios. In the LowVG, the U.S. EI imports about $0.2 \%$ of its load from Canada, predominately from Québec into ISONE and NYISO. The addition of low-operational-cost generation in the U.S. EI without similar 
additions or any thermal retirements in Canada decreases the imports from and increases exports to Canada, particularly in the RTx30 where more wind and PV generation is located in ISO-NE and NYISO. In both the RTx10 and RTx30, the U.S. EI is a net exporter to Canada. In the ITx30, a large transmission line is built between NYISO and Québec, and results in an increase in US imports from Québec. (This study does not consider any expansion of wind or solar in Canada.)

Table 45. Annual U.S. EI Net Imports from Canada

\begin{tabular}{|l|r|r|}
\hline Scenario & $\begin{array}{c}\text { U.S. El net imports } \\
\text { from Canada (TWh) }\end{array}$ & $\begin{array}{c}\text { Share of U.S. EI } \\
\text { load (\%) }\end{array}$ \\
\hline LowVG & 7.0 & 0.2 \\
\hline RTx10 & -3.5 & -0.1 \\
\hline RTx30 & -11.5 & -0.4 \\
\hline ITx30 & 4.0 & 0.1 \\
\hline
\end{tabular}

Table 46 separates the annual imports and exports that make up the annual net interchange for each region. The annual net interchange metric can mask differences if changes in imports and exports are similar in size. FRCC is a good example of a region where annual net interchange changes little across scenarios, but the utilization of the transmission between the regions increases substantially because the flows switch directions more frequently, as seen in the total amount of imports and exports in Table 46 (e.g., the total net interchange for FRCC is 4 TWh in all scenarios, but the energy that flowed across those lines increases by over a third). MISO is another case where the annual net interchange does not change significantly, but the impact of the increased transmission capability and increased VG in and around this region leads to more frequent trading with neighbors. The total imports and exports indicate increased interchange as VG penetration increases.

Table 46. Annual Imports and Exports for Each Region

\begin{tabular}{|c|c|c|c|c|c|c|c|c|}
\hline \multirow[b]{2}{*}{ Region } & \multicolumn{2}{|c|}{ LowVG } & \multicolumn{2}{|c|}{ RTx10 } & \multicolumn{2}{|c|}{ RTx30 } & \multicolumn{2}{|c|}{ IT x30 } \\
\hline & $\begin{array}{l}\text { Imports } \\
\text { (TWh) }\end{array}$ & $\begin{array}{l}\text { Exports } \\
\text { (TWh) }\end{array}$ & $\begin{array}{l}\text { Imports } \\
\text { (TWh) }\end{array}$ & $\begin{array}{l}\text { Exports } \\
\text { (TWh) }\end{array}$ & $\begin{array}{l}\text { Imports } \\
\text { (TWh) }\end{array}$ & $\begin{array}{c}\text { Exports } \\
\text { (TWh) }\end{array}$ & $\begin{array}{l}\text { Imports } \\
\text { (TWh) }\end{array}$ & $\begin{array}{c}\text { Exports } \\
\text { (TWh) }\end{array}$ \\
\hline FRCC & 1 & 5 & 1 & 5 & 3 & 7 & 3 & 7 \\
\hline HQ & 4 & 55 & 4 & 54 & 4 & 53 & 5 & 55 \\
\hline IESO & 20 & 10 & 19 & 5 & 21 & 5 & 20 & 5 \\
\hline ISO-NE & 21 & 0 & 23 & 1 & 20 & 2 & 23 & 1 \\
\hline Manitoba & 1 & 5 & 5 & 8 & 6 & 8 & 1 & 5 \\
\hline MISO & 8 & 20 & 18 & 28 & 31 & 26 & 72 & 52 \\
\hline NBSO & 13 & 7 & 15 & 8 & 14 & 5 & 12 & 4 \\
\hline NYISO & 24 & 2 & 18 & 5 & 19 & 6 & 26 & 10 \\
\hline PJM & 13 & 26 & 13 & 27 & 12 & 26 & 64 & 28 \\
\hline Saskatchewan & 2 & 0 & 4 & 0 & 5 & 0 & 3 & 0 \\
\hline SERC & 24 & 3 & 30 & 3 & 32 & 9 & 47 & 6 \\
\hline SPP & 2 & 1 & 3 & 11 & 3 & 23 & 2 & 105 \\
\hline Total & 134 & 134 & 155 & 155 & 171 & 171 & 276 & 276 \\
\hline
\end{tabular}




\subsubsection{Power Transfer Between Regions}

The energy interchange between regions gives an overview of the average direction of energy transfer over the course of the study year. However, as shown by the number of changes in the direction of net flow (Table 47), the interchange can mask a potentially large difference in how electricity flows may change within a day or season. These flows can be better represented with an analysis of the time-series transfer data and additional metrics that capture daily import-export characteristics. Figure 50 shows a two-day period of the interchange from NYISO to PJM for all four scenarios. As in Figure 49, the energy transferred annually between the regions does not change dramatically between the scenarios, but the real-time interactions do vary between scenarios. In Figure 50, increasing the level of VG leads to a larger range of interchanges, especially noticeable on June 11 in the ITx30 case, where NYISO goes from exporting nearly 2 $\mathrm{GW}$ to importing nearly $3 \mathrm{GW}$ over the span of a couple of hours. We note that, in practice, regions like PJM limit the rate and magnitude of changes for imports (PJM 2016). In this study, we did not place limits on the ramp rate for interchange. Additional work is necessary to determine whether operators would be able to accommodate the observed changes in interchange.

Table 47. Daily Number of Changes in the Direction of Net Flow between Pairs of Regions

\begin{tabular}{|l|l|l|l|l|l|}
\hline Source & Sink & LowVG & RTx10 & RTx30 & ITx30 \\
\hline FRCC & SERC & 7.4 & 8.3 & 7.5 & 6.4 \\
\hline HQ & IESO & 9.2 & 8.3 & 7.1 & 7.3 \\
\hline HQ & ISO-NE & 2.1 & 2.4 & 3.5 & 2.4 \\
\hline HQ & NBSO & 2.3 & 2.6 & 4.4 & 2.5 \\
\hline HQ & NYISO & 2.6 & 3.2 & 4.1 & 3 \\
\hline IESO & Manitoba & 9.8 & 10.3 & 8.7 & 9.7 \\
\hline IESO & MISO & 14.7 & 16.4 & 16.3 & 14.9 \\
\hline IESO & NYISO & 5.7 & 9 & 9.1 & 8.1 \\
\hline ISO-NE & NBSO & 15.2 & 14.2 & 11.7 & 11.6 \\
\hline ISO-NE & NYISO & 7.7 & 9.3 & 8 & 5.4 \\
\hline Manitoba & MISO & 5.1 & 5.7 & 5.9 & 7.6 \\
\hline Manitoba & Saskatchewan & 3.5 & 5.6 & 4.7 & 8.5 \\
\hline MISO & PJM & 4.9 & 5 & 4.7 & 3.1 \\
\hline MISO & Saskatchewan & 3.7 & 5.4 & 4.9 & 5.9 \\
\hline MISO & SERC & 12.9 & 12 & 11.4 & 11.4 \\
\hline MISO & SPP & 13.9 & 13 & 10.6 & 12.5 \\
\hline NYISO & PJM & 16.3 & 12.8 & 10.8 & 12.9 \\
\hline PJM & SERC & 9.5 & 9.2 & 7.6 & 6.6 \\
\hline PJM & SPP & NA & NA & NA & 3.7 \\
\hline
\end{tabular}




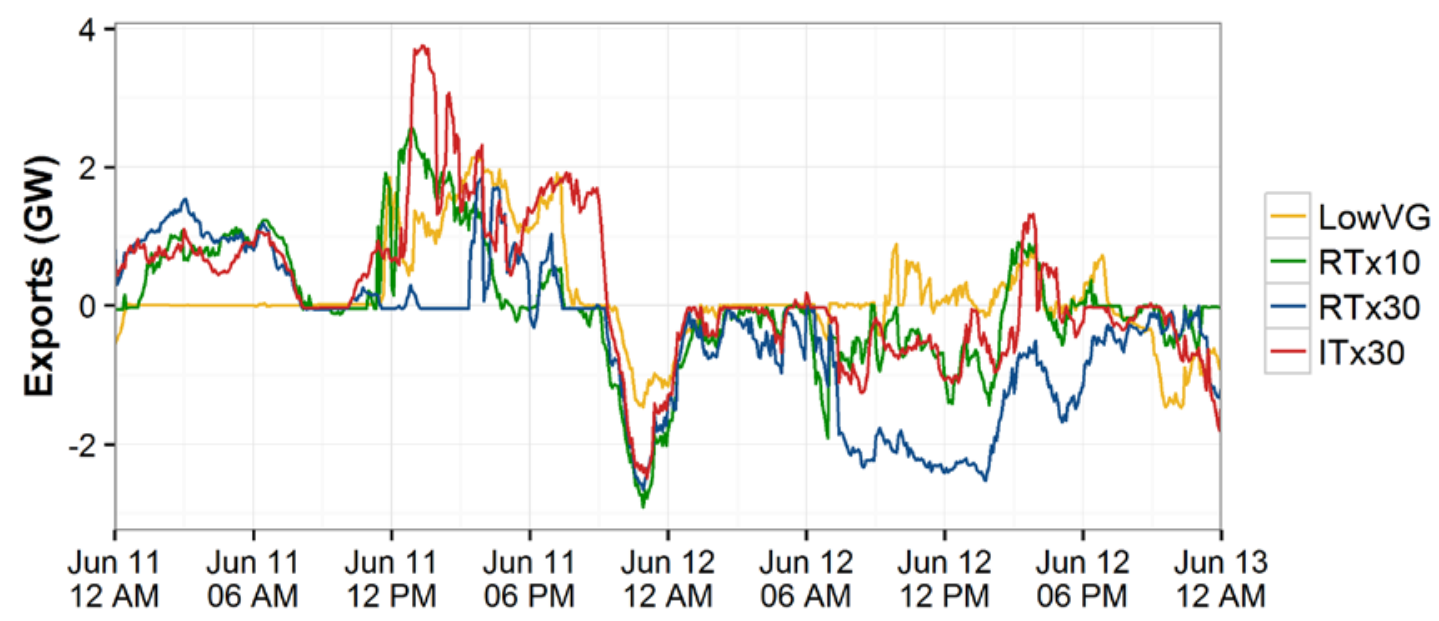

Figure 50. Exports from NYISO to PJM for all four scenarios

Figure 51 shows how the average power flow between regions changes throughout the day as more VG is added to the system. Some of the increased utilization is due to the increased transmission buildout in the EI in our four scenarios, but changes due to the high penetration of VG are also evident. For example, PV in FRCC and SERC has a large impact on regional flows during the daylight hours. Not only do exports from the Southeast to the rest of the EI increase, but in the RTx30 (the scenario with the most PV capacity) exports from MISO and the Québec Interconnection to the high load regions on the U.S. East Coast decrease due to the cheaper power coming from FRCC and SERC.

Note that some of the average flows show rapid changes, or a "saw-tooth" pattern. These rapid changes happen at the top of the hour, or when the commitment of thermal units from the dayahead schedule get enforced. Particularly in regions with quickly changing net-load conditions, the commitment of generators in the day-ahead can be significantly different from one hour to the next. This sudden up or down change, in online generation within a region leads to a reflected change in exporting and importing in these periods and causes the saw-tooth pattern. This pattern is most obvious in flows between FRCC and SERC in the high VG scenarios (Figure 51). Particularly during the afternoon net-load ramp (PV generation decreases, while load increases), FRCC shifts from importing from SERC to exporting to SERC at the beginning of the hour. This is due to CC commitment changing at the top of the hour in FRCC, and FRCC online generation changing rapidly from one 5-min interval to the next. The changing $\mathrm{CC}$ commitment can be seen in Figure 133 of Appendix E. Around the hours of PV generation, CC commitment changes rapidly, and because commitment only changes at the beginning of the hour, large changes in interchange with SERC can occur. 


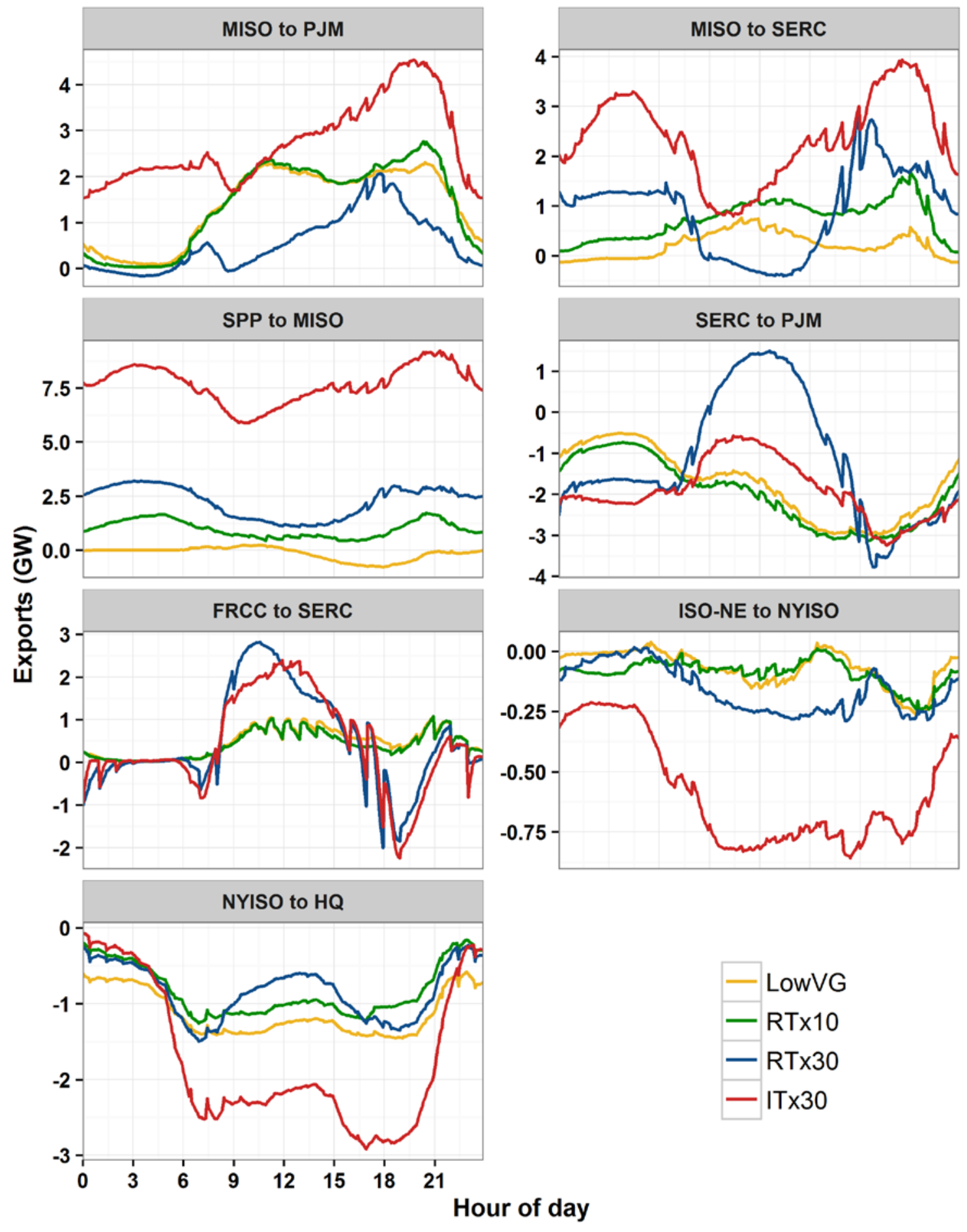

Figure 51. Average diurnal interchange between regions in the El. 
Figure 52 shows the duration curves of exports between select regions for all periods in the year. ${ }^{27}$ The biggest changes between scenarios are seen in MISO and SPP, where there are substantial transmission expansions to collect and move wind power from the west to the east, primarily to PJM and SERC. MISO exports more in the ITx30 scenario, due to increased transmission capacity and very high levels of installed VG capacity.

.As described in Section 7.3, the transmission flow limits were modeled as soft constraints, meaning the constraint could be violated at an added cost (defined by the penalty price). In figure 52 we note that some regions exceeded the soft constraints for a small percentage of the year. The presence of these violations does not indicate that the lines would have been loaded at this level in practice; instead it reveals one consequence of a simplification in the model. In reality, a local decision-maker would likely choose a different solution that kept the flows within acceptable bounds. Options to avoid violating the flow limits include redispatch of thermal generators or curtailment of VG. This, in turn, would slightly increase production costs. Furthermore, dynamic line ratings could allow temporary increases in the thermal ratings of the lines that could allow short periods of increased utilization if deemed economic.

\footnotetext{
${ }^{27}$ In a duration curve, the periods are independently sorted by magnitude, so the points at each $\mathrm{x}$-value in the four
} scenarios are not necessarily from the same time period in the time series data. 


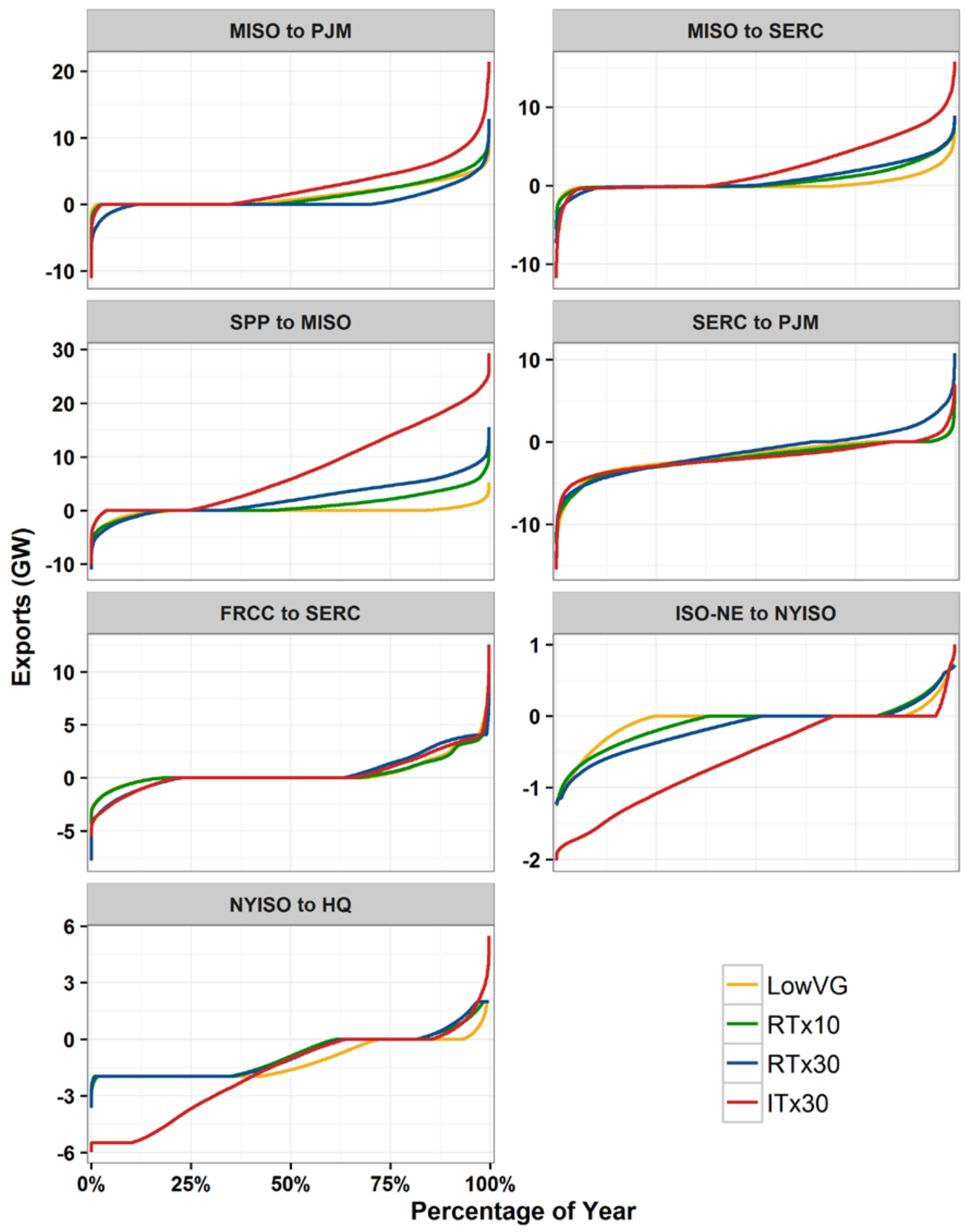

Figure 52. Annual duration curves of exports for select interfaces

Note: The y-axes differ between the plots. 
While much of the power transfer in SPP, MISO and PJM is aided by additional transmission, FRCC increases period-by-period power transfer with no transmission expansion beyond the LowVG scenario ${ }^{28}$. RTx10 does not show much change from LowVG because FRCC has no additional VG capacity in this scenario. The $30 \%$ cases show increased importing and exporting to balance the system. Figure 53, shows a more focused view ( 5 to $-5 \mathrm{GW}$ exports) of the transmission utilization between FRCC and SERC. Here, the 30\% scenarios exhibit greater utilization of the interface lines through a greater percentage of the year at above or below zero exports, as well as more energy transferred during those periods. The total energy transferred over this interface increases by more than $40 \%$, from $5 \mathrm{TWh}$ to $7 \mathrm{TWh}$, between the LowVG and the $30 \%$ scenarios (Table 46 ). This change in overall utilization has effects on the operational impacts to the individual regions regardless of the relatively unchanged net exports between scenarios. For example, even with the increased PV in FRCC in the RTx30 and ITx30, imports from SERC increase. This is likely due to FRCC utilizing SERC for resources to meet the evening net-load ramp in FRCC. Violations of the transmission flow constraint on the interfaces that connect FRCC and SERC occur $1.8 \%$ of the time in the LowVG. Adding PV in FRCC and SERC decreases transmission flow violation to $1.1 \%$ and $1.6 \%$ of all violations in the RTx30 and ITx30 respectively. Note, in today's system the interface between FRCC and SERC is constrained by other factors other than thermal limits. ERGIS did not capture those constraints and therefore assumed more import/export ability than today's system allows for all four scenarios.

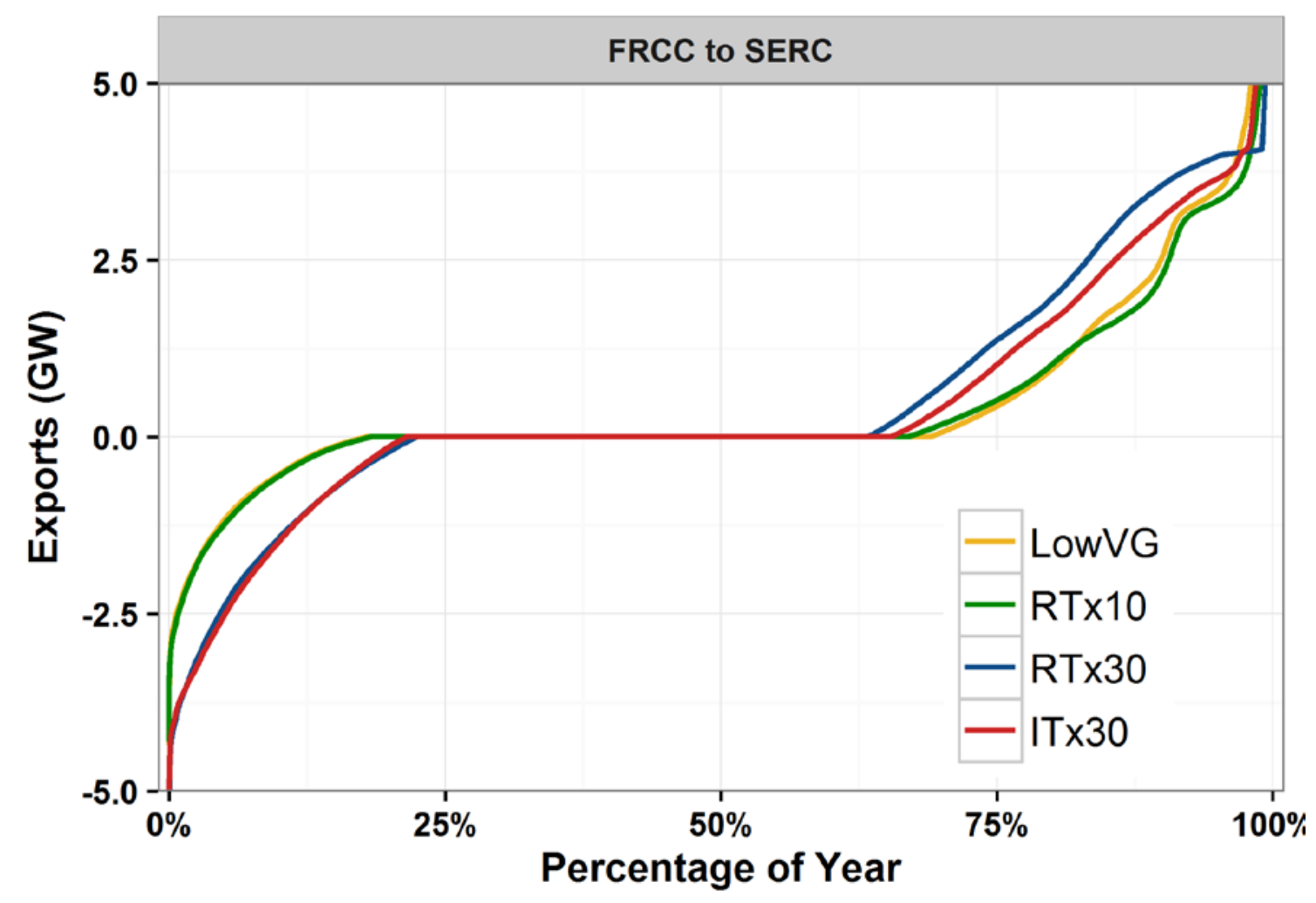

Figure 53. Annual duration curves of exports for the FRCC-SERC interface

\footnotetext{
${ }^{28}$ The transmission flowgate method for ERGIS (described in Section 7.3) creates a flow limit that is significantly larger than the current actual limit for FRCC to export to SERC.
} 


\subsubsection{HVDC Utilization in ITx30}

The transmission system is utilized differently depending on changes in the generation mix and the topology of the transmission. The topology of the ITx30 scenario includes significant expansions of HVDC lines in the MISO-PJM-SPP network (ITx30 uses the EIPC Scenario 1 expansion). Table 48 shows the number of added HVDC lines in the MISO-PJM-SPP network, which includes two lines that begin and end within MISO. Figure 54 shows the location of the HVDC lines in the MISO, SPP, and PJM regions.

Table 48. HVDC Capacity in ITx30 Scenario

\begin{tabular}{|l|l|}
\hline Interface & Added HVDC Transfer Capacity (GW) \\
\hline SPP and PJM & 2 lines x $3.5 \mathrm{GW}=7 \mathrm{GW}$ \\
\hline MISO and PJM & 2 lines $\times 3.5 \mathrm{GW}=7 \mathrm{GW}$ \\
\hline Western MISO to Eastern MISO & 2 lines $\times 3.5 \mathrm{GW}=7 \mathrm{GW}$ \\
\hline
\end{tabular}

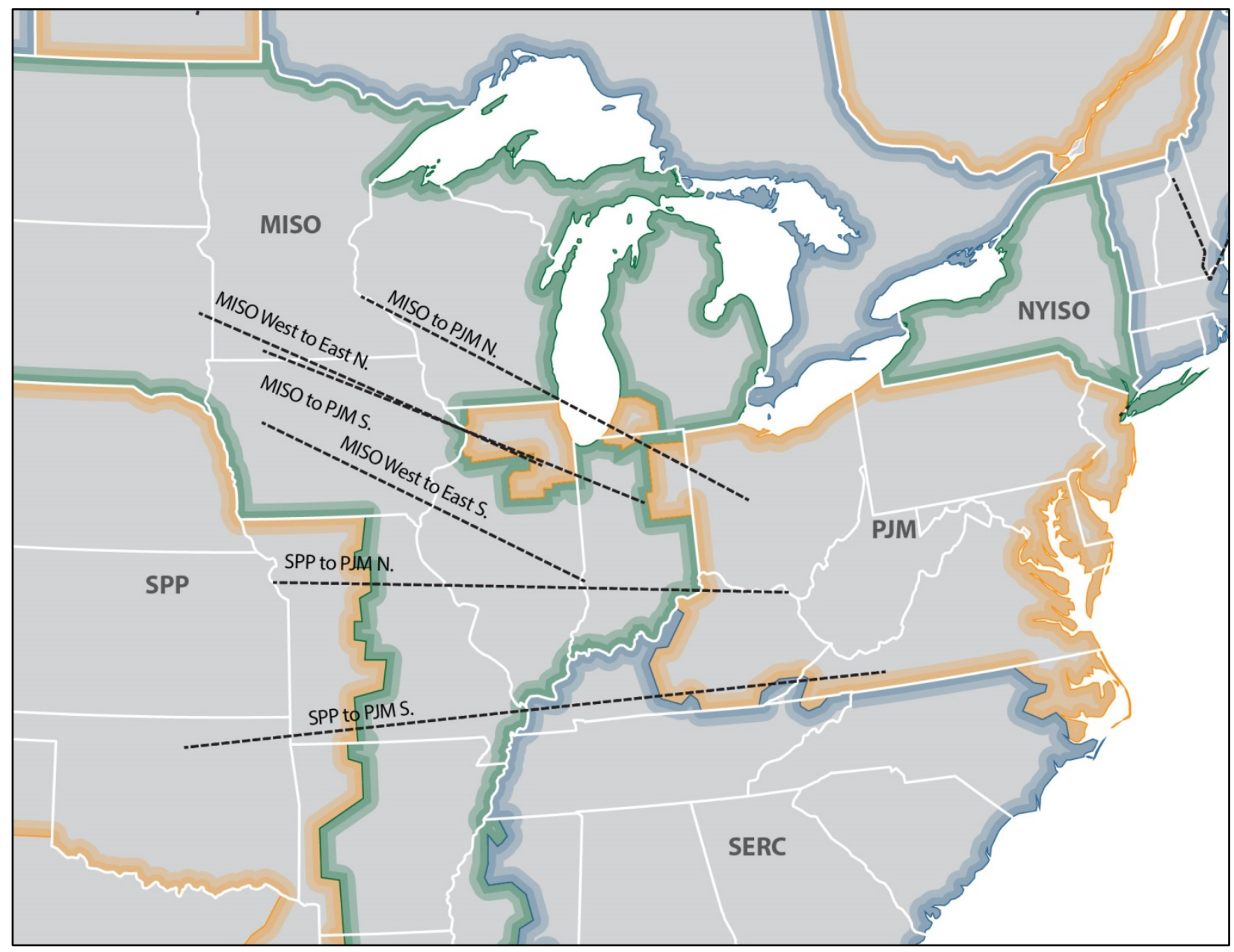

Figure 54. The ITx30 transmission expansion includes six HVDC lines connecting the MISO and SPP regions to PJM 
Figure 55 shows the annual utilization of the six HVDC lines in Table 48 for the ITx 30 scenario. The grey traces show the time series of the energy transferred on the lines, while the red traces show the duration curve of the same data. All the lines are utilized in both directions on a regular basis, as can be ascertained from the grey traces, but there is a clear trend for more exporting toward the east observed in the red trace, which follows the overall trends observed in Section 8.2.2. Several lines are using their full capacity to export east for a large portion of the year, although two lines - SPP to PJM North and MISO West to MISO East South - are used to ship power toward the west about as often as they are used to ship power toward the east.

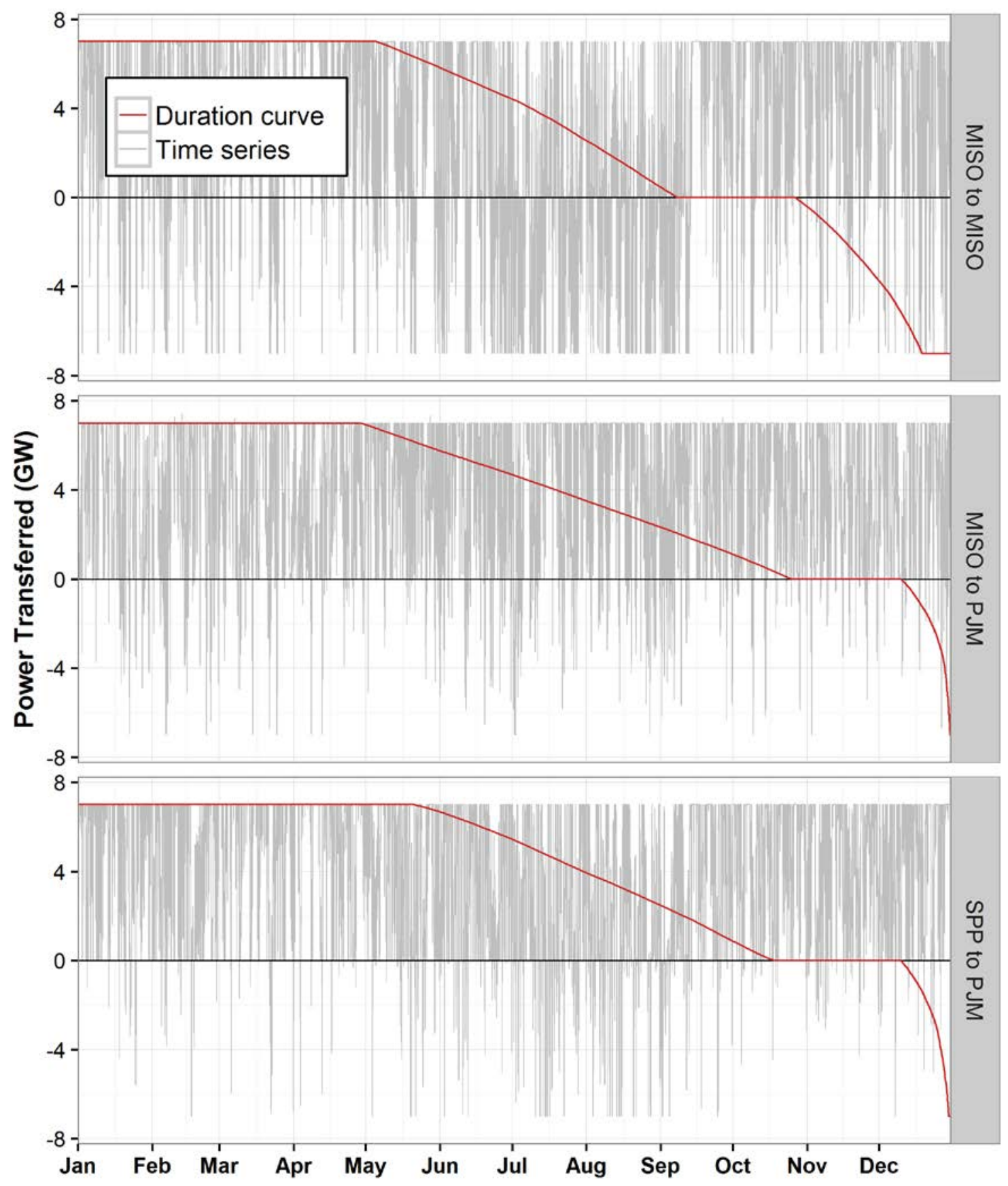

Figure 55. Energy transferred across select HVDC interties in ITx30 scenario 


\subsection{Synchronous Generation}

The dynamic performance of the power system in the presence of high penetrations of renewables is an area of active research (Eto et al. 2010, Miller et al. 2011, Miller et al. 2014, EirGrid 2011, Zhang et al. 2013). While analysis of frequency response and transient stability during contingency conditions is outside the scope of this study, UC\&ED models are critical to setting the initial conditions of dynamic modeling exercises. This study found many periods of operation in the RTx30 and ITx30 where the amount of synchronous capacity online was much lower than current levels.

Electrical system dynamics are driven in part by the inertia of online and spinning generators and from demand-side synchronous AC motors. These machines are synchronously connected to the AC network and operate at a fixed multiple of the system frequency. Any decline in the system frequency necessitates slowing of every generator or motor on the system, which helps to resist the frequency from dropping further. It is the synchronous capacity online, rather than the amount of generation from that capacity, that is important for the initial frequency response of the system, because even at part-load the entire mass of the turbine is spinning at a fixed multiple of the system frequency.

Because wind and PV generators are asynchronous to system frequency, they do not provide conventional synchronous inertia. However, active power controls for inverter-based technologies can provide very fast frequency response in the inertial timeframe and wind turbines can provide an inertial response using the spinning inertia of the turbine (Ela 2014). Furthermore, the addition of VG can lead to an increase in power system frequency response by reducing conventional generation output, leaving more headroom capacity available on those conventional units to increase output following a system disturbance (Miller 2011).

Figure 56 shows the maximum and average values for the instantaneous VG generation as a percentage of total online capacity. All regions show significant increases in the both the average and maximum VG capacity penetrations, which implies reductions in the synchronous (non-VG) capacity penetrations. FRCC and SPP have maximum VG capacity penetrations of more than $65 \%$, and the U.S. EI as a whole achieves a maximum of $47 \%$. None of the extreme periods observed in this section have been analyzed using appropriate dynamic stability or AC power flow analytical tools. As such, these results are provided to identify areas for future research on system stability in the EI. 


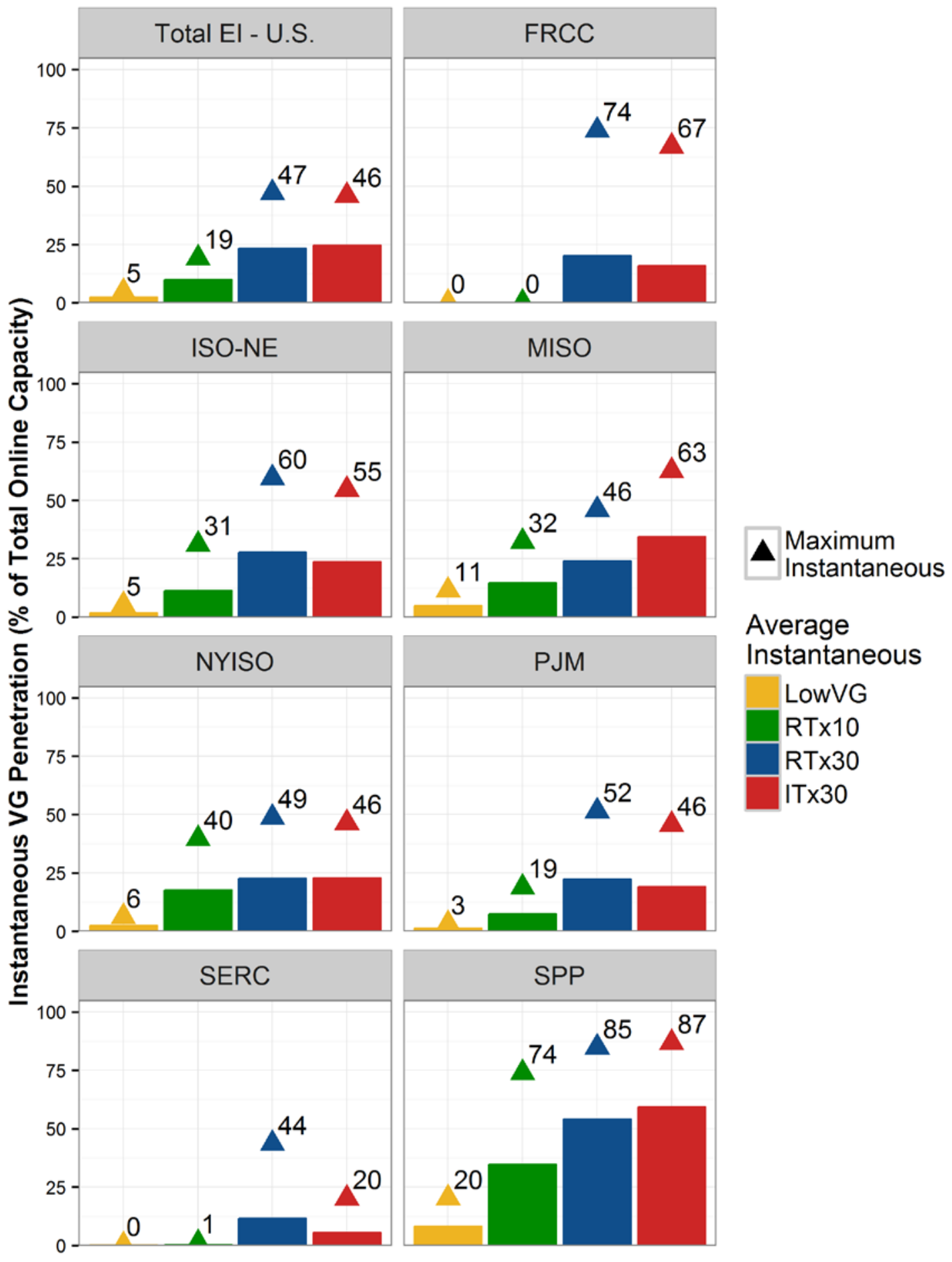

Figure 56. Instantaneous VG penetration as a percent of online capacity

The maximum and average number is presented, with the value on the plot representing the maximum. 


\subsection{Daily Operations}

We analyzed the daily operation of the power system at a regional and system wide level for an entire year of operations to understand how the unit commitment and 5-minute dispatch of the system are impacted by wind and PV. Three periods were selected from the annual solution for detailed analysis of system operations - high load, high VG, and high net load ramp. These periods were analyzed in terms of committed capacity, generation, VG curtailment, and interregional transfers.

In order to understand how forecast errors and sub-hourly variability impact operations, we developed a set of plots to show the difference between what was committed in the day-ahead and what was available and dispatched in the real-time. Figure 57 describes how to read these plots. For thermal resources, the dark gray line is the capacity committed in the day-ahead market for each class of thermal resources. This data is a result of the day-ahead, hourly unit commitment conducted in PLEXOS. For wind and PV, the dark gray line represents the dayahead forecast but does not include any VG curtailment scheduled in the day-ahead. The colored curves and lines represent the real-time (5-minute) operation. In real-time, at a 5-minute level, both coal and CC units can be dispatched to any point within their commitment schedule.. Since $\mathrm{CT} / \mathrm{Gas}_{\text {boiler }}{ }^{29}$ units are the only thermal resources that can be committed or decommitted in real time, the real-time committed capacity can differ from the day-ahead committed capacity. The difference between the gray line and the pink line for CT/Gas boilers is the difference in committed capacity. As with other thermal plants, the filled area is the amount of energy generated by the resources. The blue and yellow lines for wind and PV reflect the actual potential output of the wind and PV resources at a 5-minute level. Any differences between the potential output (the blue and yellow lines) and the generation (the blue and yellow shaded regions) for wind and $\mathrm{PV}$ is curtailment of the resource.

A collection of the scripts developed to create the graphs in Figure 57, and several other figures in this report are being published for the public on GitHub. ${ }^{30}$ Collectively, these scripts are referred to as the Multi-Area Grid Metric Analyzer (MAGMA). This toolkit consists of scripts that develop graphs for dispatch stacks, zonal data, VG curtailment, key period analysis, interchange, production costs, and commitment. These scripts can be modified by users to work with results from other PLEXOS simulations and could be amended to work with other modeling packages as well. By making these tools public, we hope to assist the broader research community in enabling timely, reproducible, and accurate data for decisions makers.

\footnotetext{
${ }^{29}$ While we have bundled CT and Gas boiler plants into one category for easier discussion and visualization, only CT plants can be recommitted in real time.

${ }^{30}$ Links to the GitHub repositories for the MAGMA code are available at: www.nrel.gov/ERGIS
} 


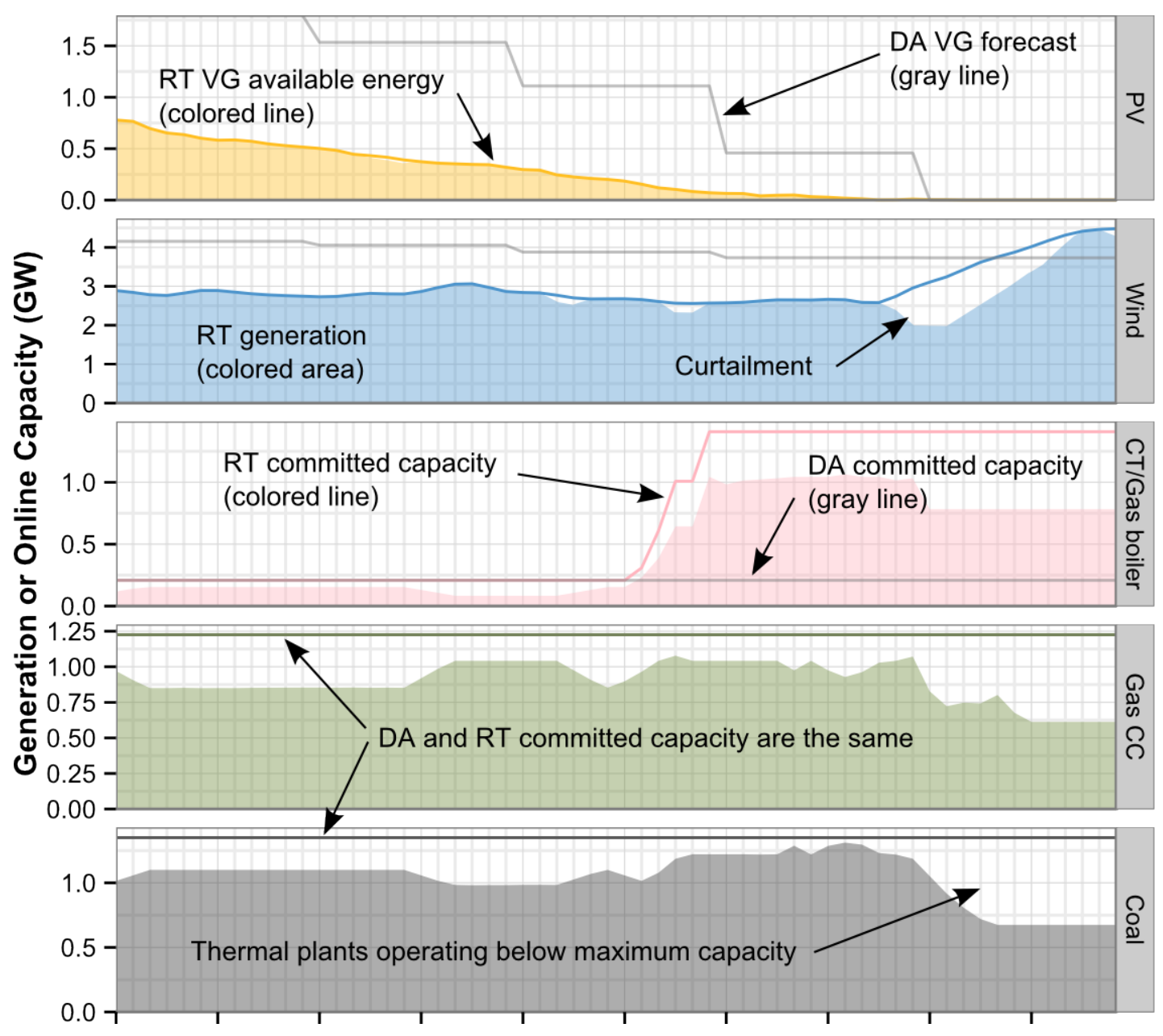

Figure 57. Key for interpreting day-ahead and real-time dispatch and commitment figures

To better understand how the net load impacts interchange, we created tools to visually display the power system data in ways that enable deeper multi-area analysis. These tools can provide additional insights and help identify data and modeling errors not easily observed in static visualization. The R statistical software package was used to create a collection of scripts called "kaleidoscope". Kaleidoscope consists of three types of visualization (see Figure 58): a geographic diagram, a chord diagram, and dispatch charts. The geographic diagram provides a qualitative view of the study domain representing individual generator output and interregional transmission flows. The output of every generator is plotted for every five minutes as semitransparent bubbles with areas proportional to their associated output, sorted by size, and colored by generation type. This approach was adopted from a visualization created for the Renewable Electricity Futures Study (Hand, et al, 2012). A single frame provides an overall understanding of the geographic distribution of generation, and when animated, the dynamics of that distribution. Arrows overlay the generation bubbles, representing regional transmission flows and provide a sense of the direction in energy flows. The chord diagrams provide a more quantitative view of the net interchange between each of the regions. Chord diagrams were designed and are primarily used for comparative genomics (Krzywinski, et al. 2009), but are well suited to show interchange between regions. Each region is represented as an arc on the perimeter of a circle; the length of the arc is proportional to that region's total interchange in 
GW. To the extent practicable, regions that share a common border were positioned on opposite sides of the circle. Directional chords are drawn between two regions to represent the direction and magnitude of interchange. The colors of the chords match their source. The width of each chord end-point reflects the interchange magnitude; wider chords represent greater interchange. Finally, kaleidoscope provides methods to display aggregate generator output for each region and scenario as bar charts or grouped stacked bar charts.


Figure 58 A screen shot of the kaleidoscope visualization suite as designed for the 14 Mpixel display in the NREL Insight Center. Left: geographic generation and flow diagrams, center: net interchange chord diagrams, right: dispatch stacked bar charts.

We created animations for each of the three periods discussed in the following subsections ${ }^{31}$

\subsubsection{High Load Conditions}

High load conditions for the system occur between July 31 and August 3. The peak load during this period is approximately $700 \mathrm{GW}$. In this section we focus our analysis on the behavior of generation during high load conditions. Figure 60 shows the 5-minute dispatch for the entire study footprint. In the LowVG, peak demand is met primarily with thermal generation. Significant amounts of CT/Gas boiler generation are used. The quantity of thermal generation, particularly CT/Gas boilers decreases as wind and PV are added to the system, and shifts toward the evening as the PV penetration level increases in the RTx30 and ITx30. Wind and PV resources contribute significant energy to meeting the peak load in both RTx30 and ITx30. However, the net load peak ${ }^{32}$ in the RTx30 is steeper than the ITx30 because of the evening ramp associated with PV in the RTx30.

High PV penetration also impacts operation of pumped storage and hydro generating resources. In the RTx30 and ITx30, hydro generation and pumped storage generation shifts to later in the day than in the lower penetration LowVG and RTx10 scenarios. Dispatch of coal and CC resources also changes in the $30 \%$ scenarios. Overnight ramping is most significant in the ITx 30 ,

\footnotetext{
${ }^{31}$ A sample of the kaleidoscope visualization for large displays is available at our website: www.nrel.gov/ERGIS.

${ }^{32}$ Net load is load minus wind and solar generation. The net load in these figures is the line below the blue wind generation area.
} 
where wind output is highest. In the RTx30 we observe a short-term ramp in the early morning before sunrise. After sunrise, coal generation is displaced by PV generation. At sunset in the $30 \%$ scenarios, CT/Gas boilers are ramped to meet the peak net load. In all scenarios, the U.S. EI relies on power imports from the Canadian provinces to meet peak load and peak net load.

Figure 61 shows the difference between the day-ahead commitment of fossil fueled resources, day-ahead forecast of wind and PV generation, and the real time commitment and generation of those resources during peak load for the entire study system. A forecast error for wind generation occurs on July 31 and again on August 1 in the RTx10, RTx30 and ITx30 cases. This error is most severe in the ITx30. The forecast error results in the over commitment of CTs in the realtime. Compared to the RTx30, the ITx30 also shows fewer CT/Gas boiler commitments and less $\mathrm{CT} /$ Gas boiler generation for the period of July 31 -August 3 . While the timing of coal and CC generation is largely constant across scenarios, the magnitude and steepness of the ramps increases in the RTx30 and ITx30. We also note a small ramp in coal and CC generation before sunrise. Even during peak load conditions, high penetrations of wind and PV largely displace thermal plant generation, compared to the LowVG and RTx10. Hydro plants shift production to sunset hours as the amount of PV increases on the system.

Analysis of the regional commitment and dispatch helps to identify regional impacts. Figure 62 and Figure 63 show that regional forecast errors in SPP and MISO drive system wide VG curtailment on July 31 for the RTx30. The CC and CT/Gas boiler dispatch stack for SPP is very shallow, with nearly zero capacity from these resources online in the RTx30 on July 31 . When wind generation exceeds forecast, the system will accept the additional wind as the lowest cost available option and redispatch committed coal and $\mathrm{CC}$ units down as far as their minimum generation levels will allow.

Power systems in neighboring MISO and PJM behave differently during the same time period. Commitments of CT/Gas boilers in MISO (Figure 63) and PJM (Figure 64) change as the PV generation increases. Compared to the LowVG and RTx10, CT/Gas boiler generation in the RTx30 and ITx30 is shifted to later in the day. The pronounced ramp in CT/Gas boiler generation in Figure 60 is generally coincident across regions, with the majority of the CT/Gas boiler generation in MISO and PJM. The increase in CT/Gas boiler generation in these regions on July 31 is a byproduct of the day-ahead commitment for coal and CCs, both of which are dispatched in real-time at a level nearly equivalent to their commitment level.

In the eastern United States, PJM, ISO-NE, and NYISO experience forecast errors during this high load period that impact interconnection-wide results. However, the forecast errors for these regions occur around midnight on both August 1 and August 2, and are not coincident with forecast errors in SPP and MISO. These similarities in U.S. EI wind forecast error can be seen in the wind generation of Figure 60 for the RTx30. Figure 64, Figure 65, and Figure 66, illustrate an underestimate of wind for the day-ahead commitment in PJM, ISO-NE, and NYISO on July 31, and a more significant underestimate for wind on August 1.

PJM, ISO-NE, and NYISO have significant quantities of CC and CT/Gas boiler generation online during the August 1 forecast error period, which allows thermal resources to be dispatched below the day-ahead commitment to accommodate the unexpected wind generation. In SPP, on July 31 very little CT/Gas boiler and CC generation online, coal units are unable to 
accommodate the excess wind, and the system must curtail wind and PV to balance regional load. Conversely, in MISO, the ability of the system to accommodate under-forecast wind generation on July 31 is limited, in part, by transmission capacity between PJM and MISO. In FRCC, CC plants operate at day-ahead commitment levels during the night time hours, and CC and coal cycle during hours of PV generation for both the RTx30 and ITx30 (Figure 67). As seen in Figure 67, FRCC exports to SERC in all scenarios and experiences modest amounts of PV curtailment in the RTx30.

Analysis of this period of high load indicates that wind and PV can play a role in meeting peak load conditions. Since there are significant amounts of thermal generation online during these high load conditions, there is considerable down ramp capability available to accommodate under forecasts for wind in the day-ahead. Analysis of this period also shows that new patterns of operation will be required of thermal and hydro resources during high load conditions. Compared to the LowVG and RTx10 case, operations in the RTx30 and ITx30 show flexible resources like hydro, pumped storage and CT/Gas boilers shifting generation to later in the day to accommodate the down ramp of PV caused by the setting sun. Wind generation persists across all hours of this time period and peaks during the evening hours. While this may suggest that the diurnal patterns of wind and solar are a natural complement to one another in the EI, additional analysis is necessary to determine the prevalence of this behavior across multiple years and a changing climate. Similarly, further analysis of the availability of all resources during peak load and other challenging conditions is also necessary. Since wind and PV alter the operation of the rest of the generation fleet, it is unclear from this analysis whether all resources assumed in our study are likely to remain revenue sufficient to meet adequacy needs. 

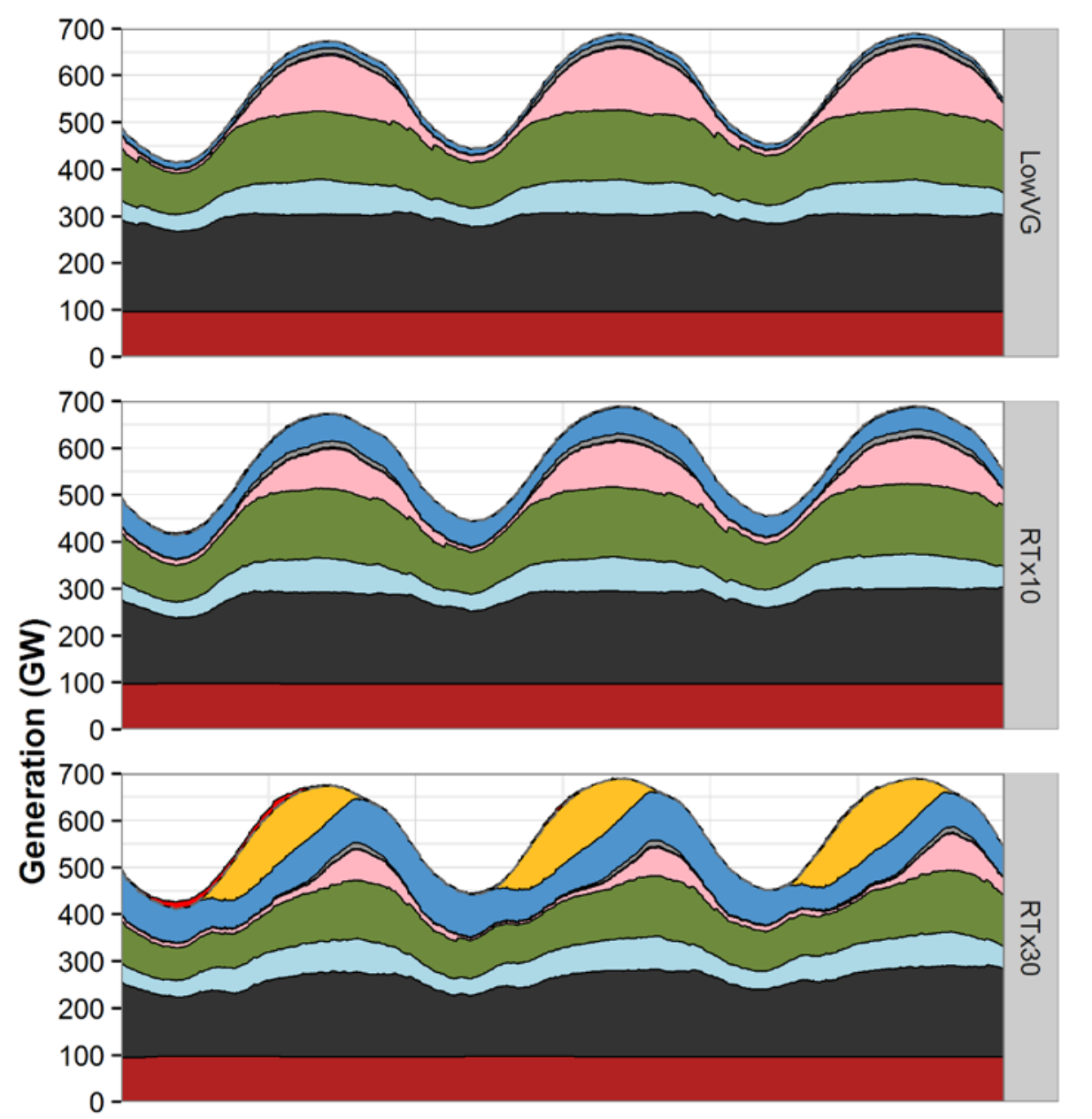

Load

Curtailment

PV

Wind

Pumped Storage

Other

CT/Gas boiler

Gas CC

Hydro

Coal

Nuclear

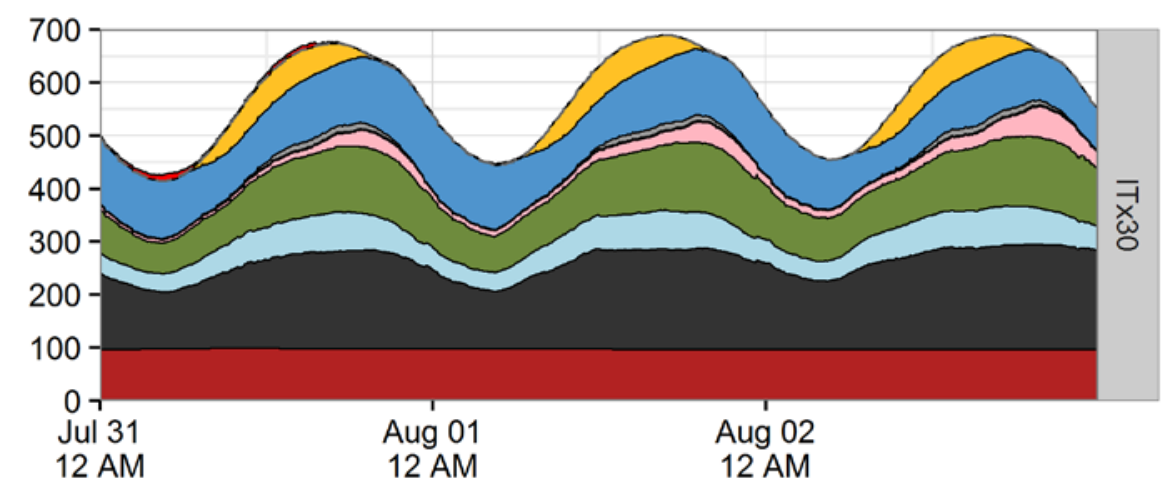

Figure 59. System dispatch in each scenario for the El during high load conditions. 


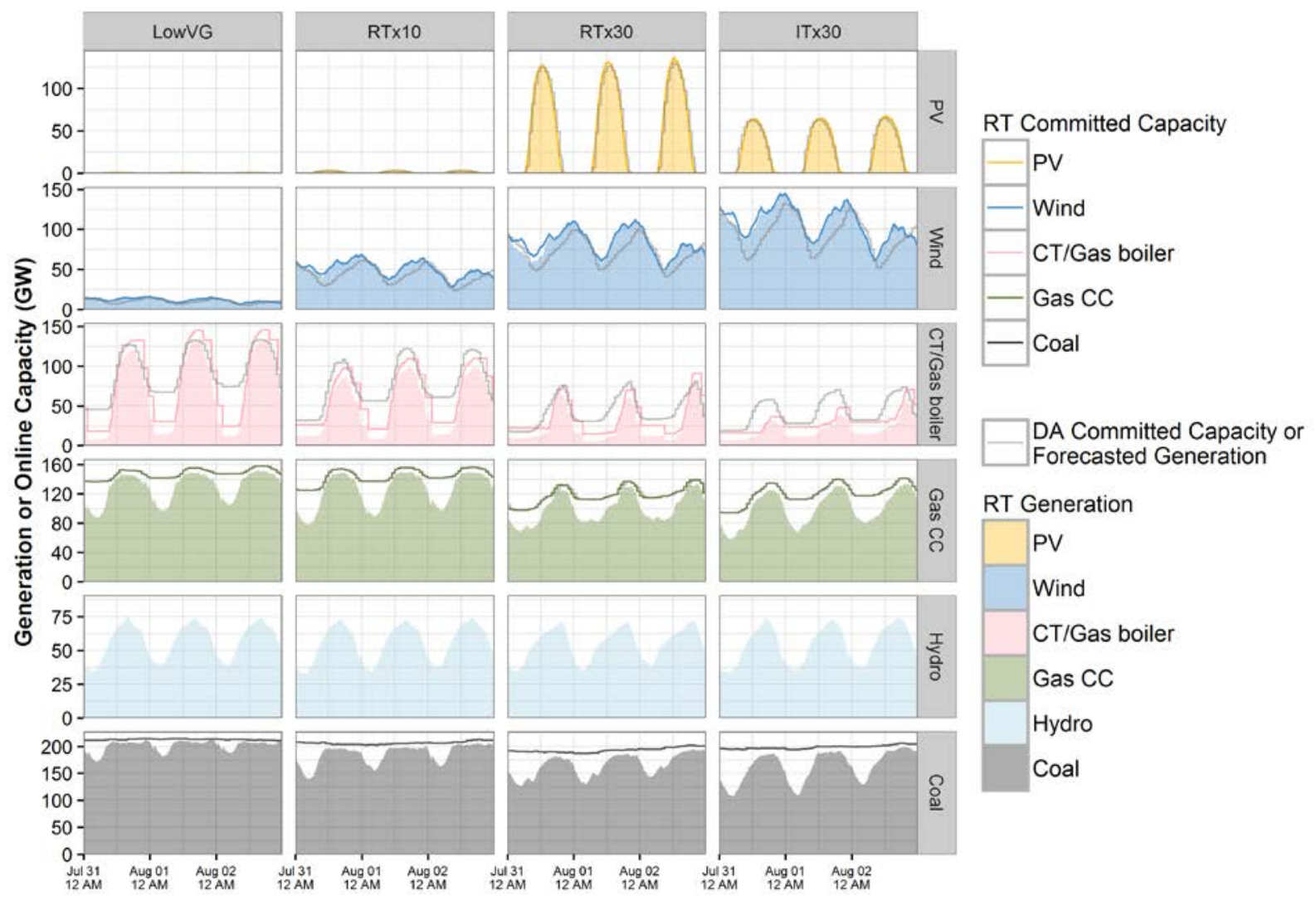

Figure 60. Combined commitment and dispatch of the El for PV, wind, CT/Gas boiler, CC, hydro, and coal during high load conditions.

For a guide to interpreting this plot, please refer to Figure 57. 


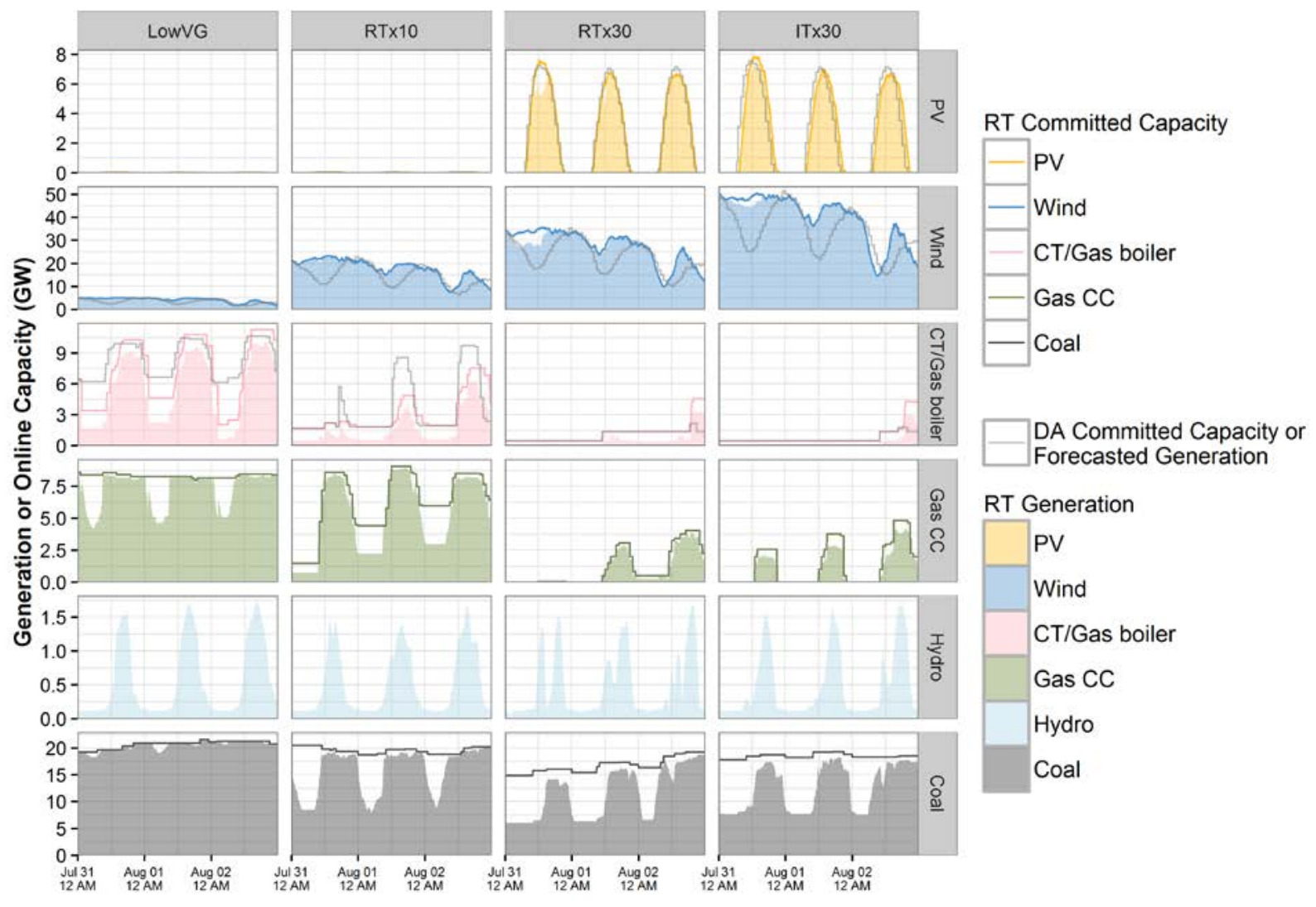

Figure 61. Combined commitment and dispatch of SPP for PV, wind, CT/Gas boiler, CC, hydro, and coal during high load conditions.

For a guide to interpreting this plot, please refer to Figure 57. 


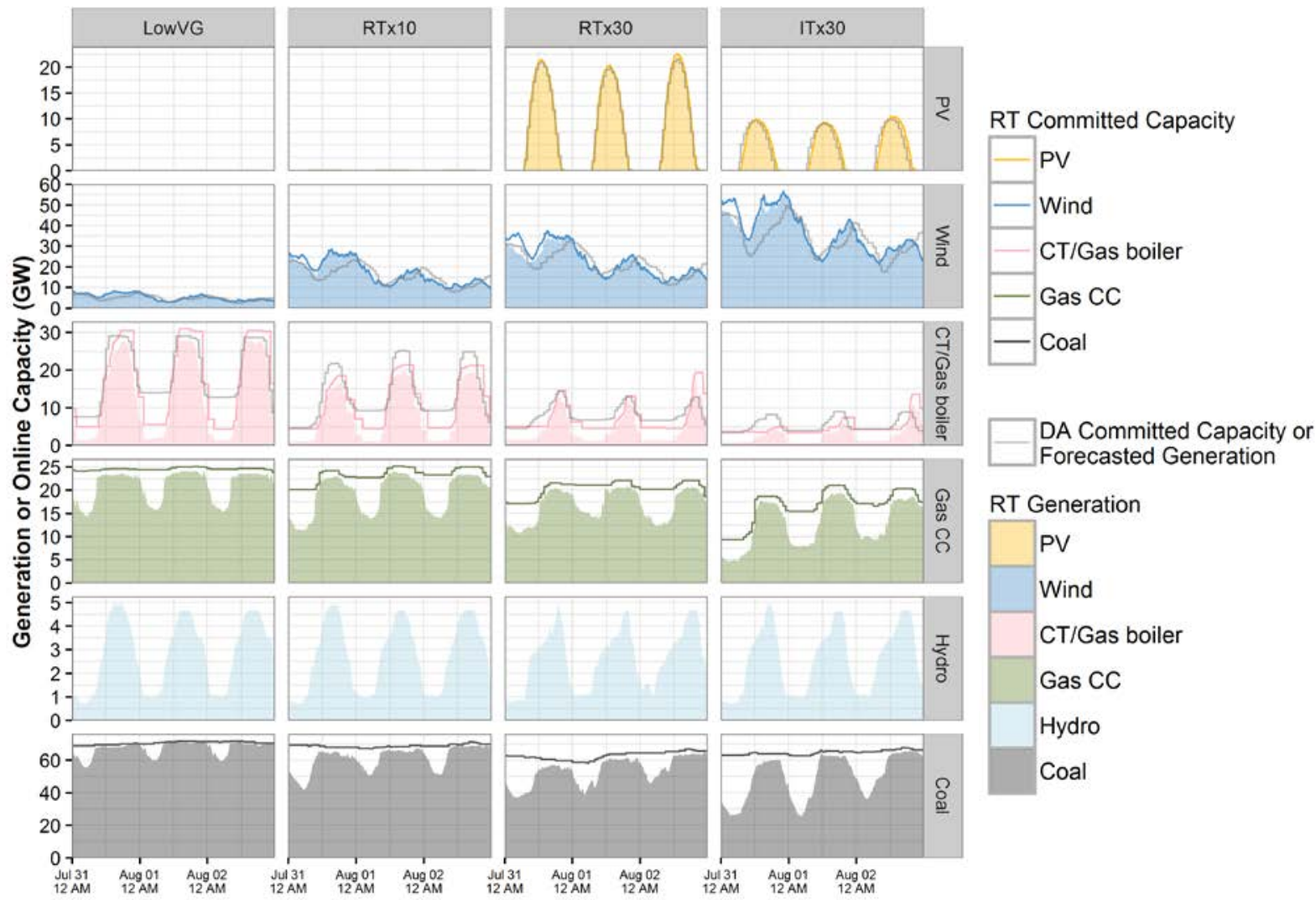

Figure 62. Combined commitment and dispatch of MISO for PV, wind, CT/Gas boiler, CC, hydro, and coal during high load conditions.

For a guide to interpreting this plot, please refer to Figure 57. 


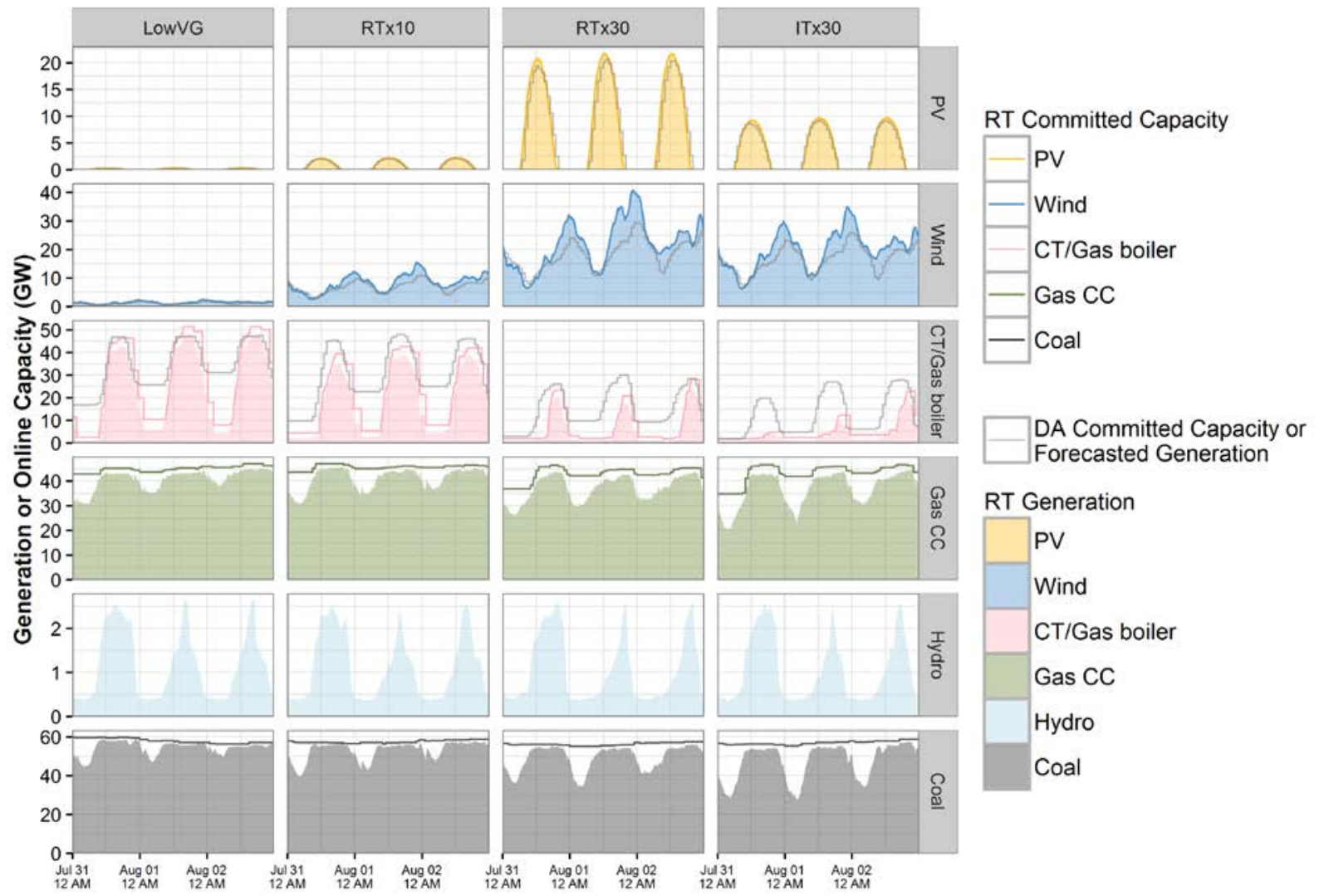

Figure 63. Combined commitment and dispatch of PJM for PV, wind, CT/Gas boiler, CC, hydro, and coal during high load conditions.

For a guide to interpreting this plot, please refer to Figure 57. 


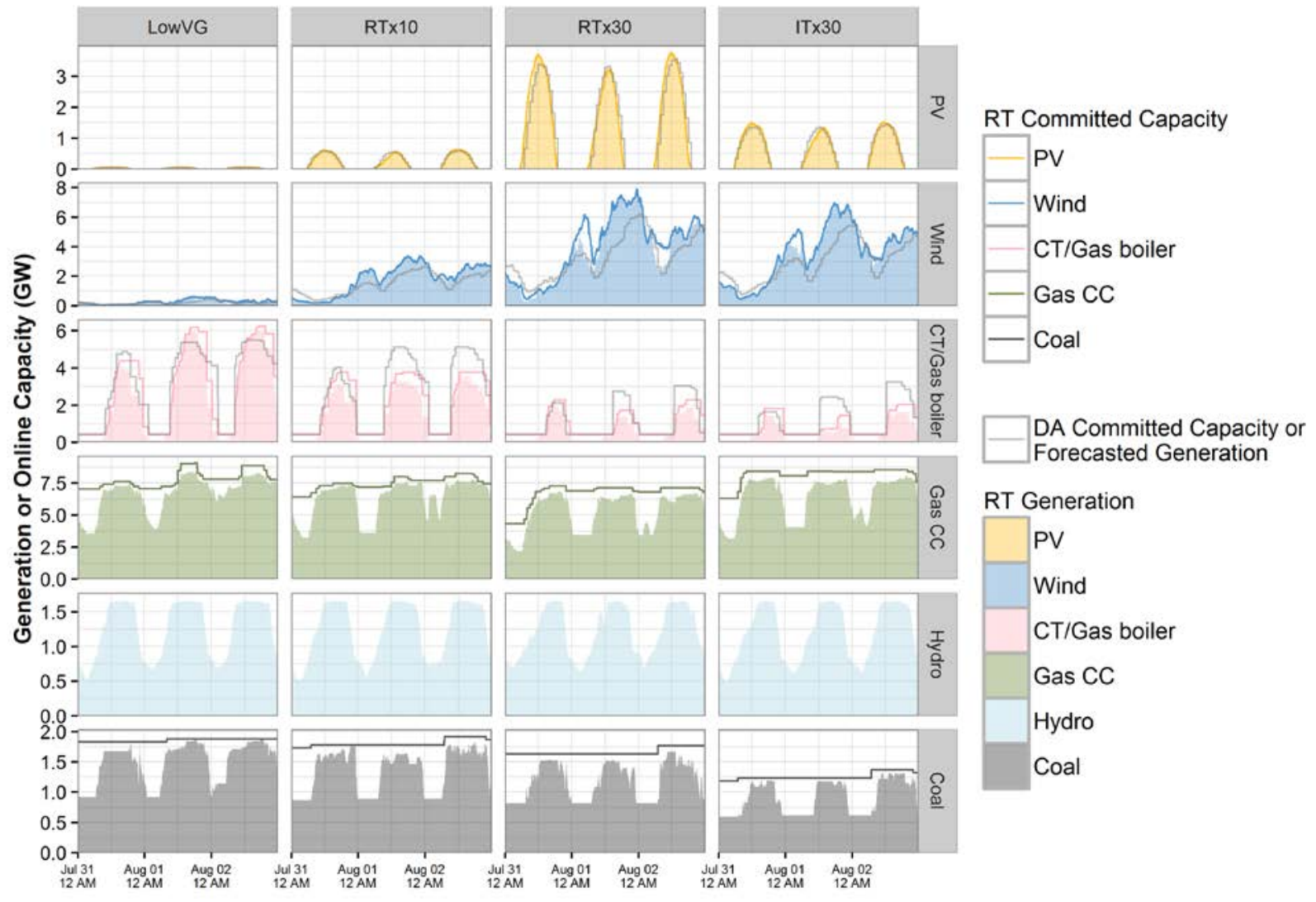

Figure 64. Combined commitment and dispatch of ISO-NE for PV, wind, CT/Gas boiler, CC, hydro, and coal during high load conditions.

For a guide to interpreting this plot, please refer to Figure 57. 


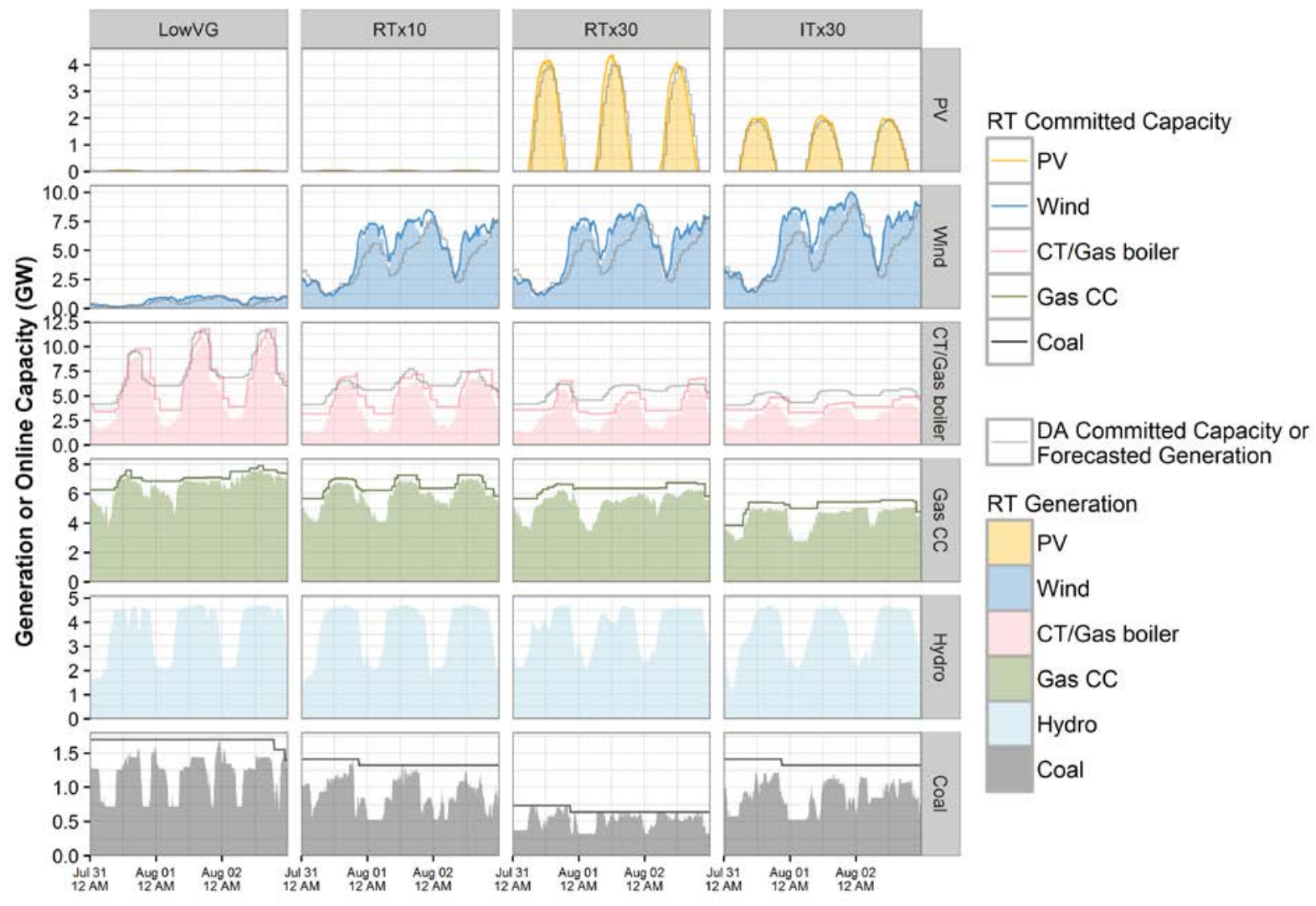

Figure 65. Combined commitment and dispatch of NYISO for PV, wind, CT/Gas boiler, CC, hydro, and coal during high load conditions.

For a guide to interpreting this plot, please refer to Figure 57. 


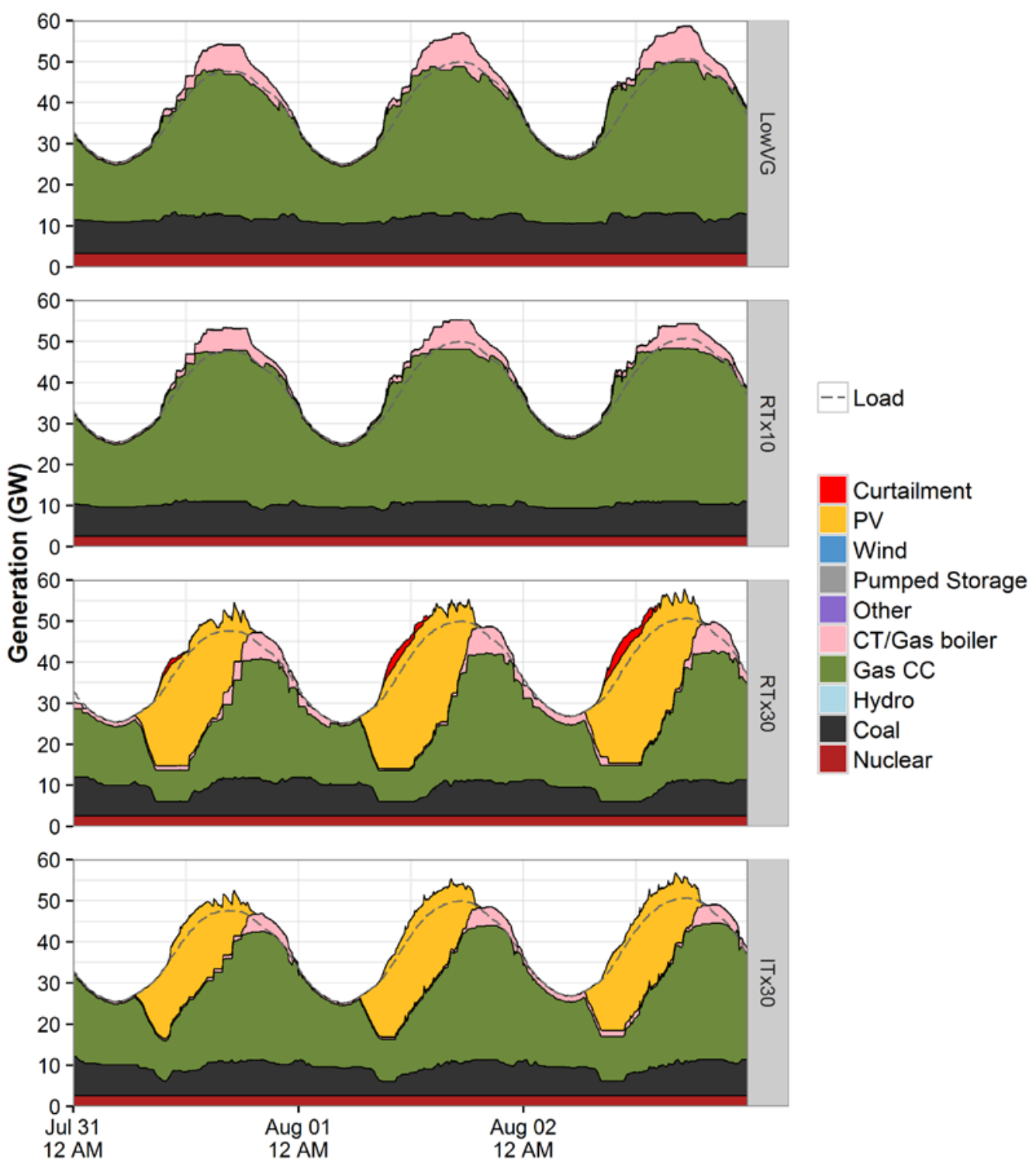

Figure 66. System dispatch in each scenario for FRCC during high load conditions.

\subsubsection{High VG Conditions}

In Section 5.2, the input data sets for wind and PV were analyzed to identify annual and monthly patterns. This analysis indicated that the periods of highest potential generation from wind and PV occurred in the spring and fall. Based on this analysis, we selected a period of high combined wind and PV generation to determine how it impacted the commitment and dispatch of the system as a whole, and several regions in particular. Additional attention was paid to transmission flows between the regions to understand the importance of regional coordination in managing the variability and uncertainty of wind and PV.

The period selected for this analysis was May 11 through May 13. Figure 68 shows the 5-minute dispatch for the entire system during this time period. Total demand varies between approximately $300 \mathrm{GW}$ and $425 \mathrm{GW}$. In the LowVG, demand is met predominately using coal, nuclear, and CC generation. Small amounts of wind and CT/Gas boiler are visible, and the 
pattern of thermal dispatch follows traditional patterns that peak during the day and are lowest at night. The lowest load occurs the morning of May 13. Figure 68 includes all Canadian provinces in the study and details how hydro plant operation changes across the scenarios. In the RTx10, nearly $60 \mathrm{GW}$ of wind generation is on the system (Figure 69). The wind in the RTx10 displaces both gas and coal generation that were online in the LowVG case. A small amount of VG curtailment in the RTx10 is observed on the evening of May $11^{\text {th }}$ during low net load levels.

In the RTx30 and ITx30, significantly more wind and PV generation is on the system. In the RTx30, PV and wind generation both peak at around $100 \mathrm{GW}$ each, and on some occasions the coincidence of wind and solar generation amounts to nearly $200 \mathrm{GW}$, as seen in Figure 69. In the ITx30, PV generation peaks at about $60 \mathrm{GW}$ and wind generation peaks at almost $150 \mathrm{GW}$. On May 11 in the ITx30, the combined generation of wind and solar is more than $170 \mathrm{GW}$. VG curtailment during this time period, Figure 68, is fairly consistent between the RTx30 and ITx30 scenarios, however we note more pronounced peaks in the VG curtailment in the RTx30 around noon.

Low load conditions have historically been a challenging period for system operations. Often times, thermal generators must be turned to very low operating levels, or de-committed completely to balance system demand. Compounding this challenge, which historically occurred during the early morning hours, is the need to quickly ramp thermal resources to meet daily load patterns. In ERGIS, we observe a substantial shift in minimum net load events. When compared to the LowVG case, the minimum net load is nearly $100 \mathrm{GW}$ lower in the RTx30 and ITx30, and on May 12, the RTx30 case experiences two very low net load periods within 12 hours.

Figure 68 shows the system dispatch for all scenarios during this period. The net load shape in this figure differs significantly from net load curves observed in the California Independent System Operator (CAISO) "Duck Chart". ${ }^{33}$ The "duck" shape in the CAISO net load is driven largely by high PV penetration. In the ERGIS scenario, the combination of wind and solar creates two periods of low net load - in the early morning driven by wind, and in the early afternoon, driven by solar. By analyzing regional dispatch stacks, Figure 69-Figure 72, we see that the regions driving the low net load in the early morning are MISO and PJM. At 13:25, however, the low net loads are driven by very high solar outputs in FRCC and SERC. At 03:30 the net load is at its lowest, $195 \mathrm{GW}$, and at 13:25 it is $200 \mathrm{GW}$.

In Figure 71 and Figure 72, we see that SERC and FRCC have significant amounts of PV. SERC is able to export that PV to neighboring MISO and PJM, while FRCC must curtail approximately $10 \mathrm{GW}$ of PV. ${ }^{34}$ By comparing the results for FRCC and SERC for the ITx30 and RTx30 we see how differences in PV availability impacts operations. In the ITx30, SERC has relatively low levels of PV compared to FRCC. This allows FRCC to export more energy to SERC. However, in the RTx30 scenario, FRCC and SERC have much more similar PV resource, reducing economic attractiveness of exports from FRCC, leading to PV curtailment.

In the videos created using our kaleidoscope tools, we observe how these events impact regional interchange. Figure 73, part of a kaleidoscope visualization, shows the net interchange for the

\footnotetext{
${ }^{33} \mathrm{https} / / /$ www.caiso.com/Documents/FlexibleResourcesHelpRenewables_FastFacts.pdf

${ }^{34}$ Link to a full video of this period, for each scenario at www.nrel.gov/ERGIS.
} 
RTx30 at 03:30 during the minimum net load for the system. Here we see that high wind conditions exist across the interconnection and result in significant exports from ISO-NE, NYISO, MISO, PJM, and SPP. Both SERC and Canada are heavy importers. Shortly after sunrise, around 7:00, a change in the direction of flows is observed across the system. SERC and Canada become exporters in the morning, and by 13:25 (Figure 74) the system has reached a second very low minimum net load of $200 \mathrm{GW}$. Finally, at sunset the flows begin to change directions again as thermal and hydro plants are dispatched to meet the evening ramp and accommodate a wind forecasting error. Significant changes in commitment, dispatch, and interchange at sunrise and sunset were present across several time periods. These changes were not as common in LowVG case and suggest that additional attention may need to be paid to manage diurnal patterns that deviate from current operations. 

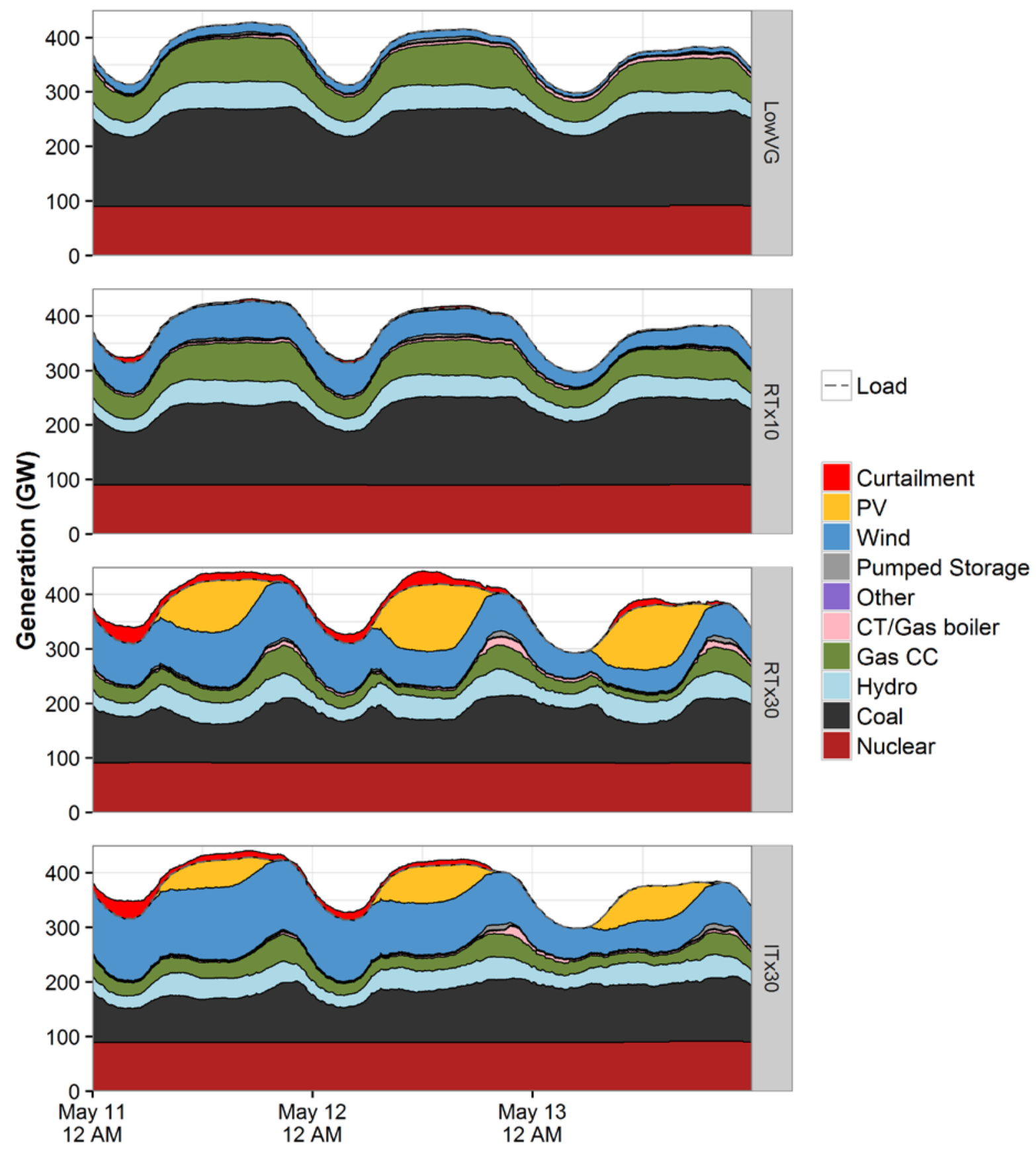

Figure 67. System dispatch in each scenario for the El during high VG conditions. 


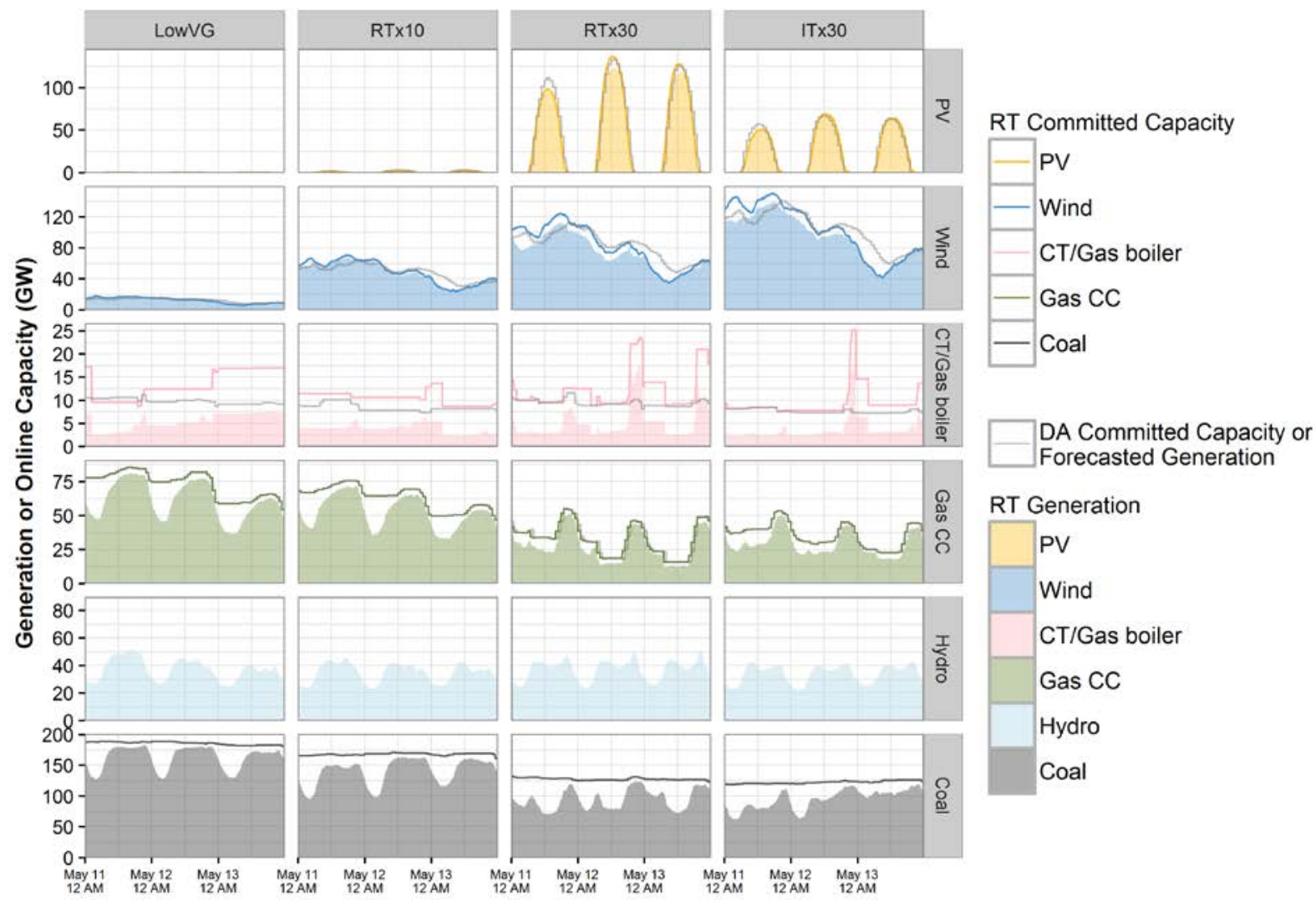

Figure 68. Combined commitment and dispatch of the El for PV, wind, CT/Gas boiler, CC, hydro, and coal during high VG conditions.

For a guide to interpreting this plot, please refer to Figure 57. 

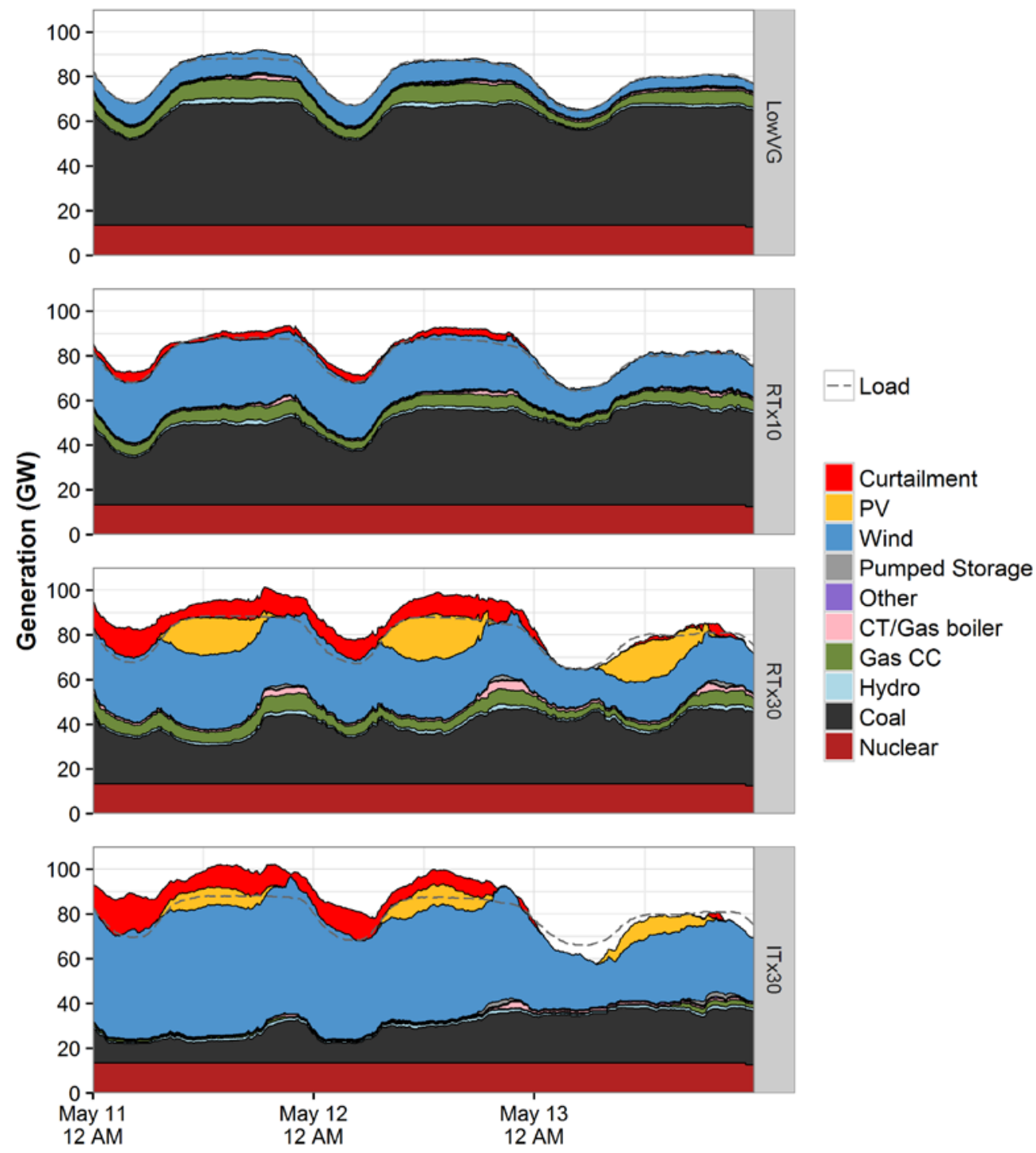

Figure 69. System dispatch in each scenario for MISO during high VG conditions. 

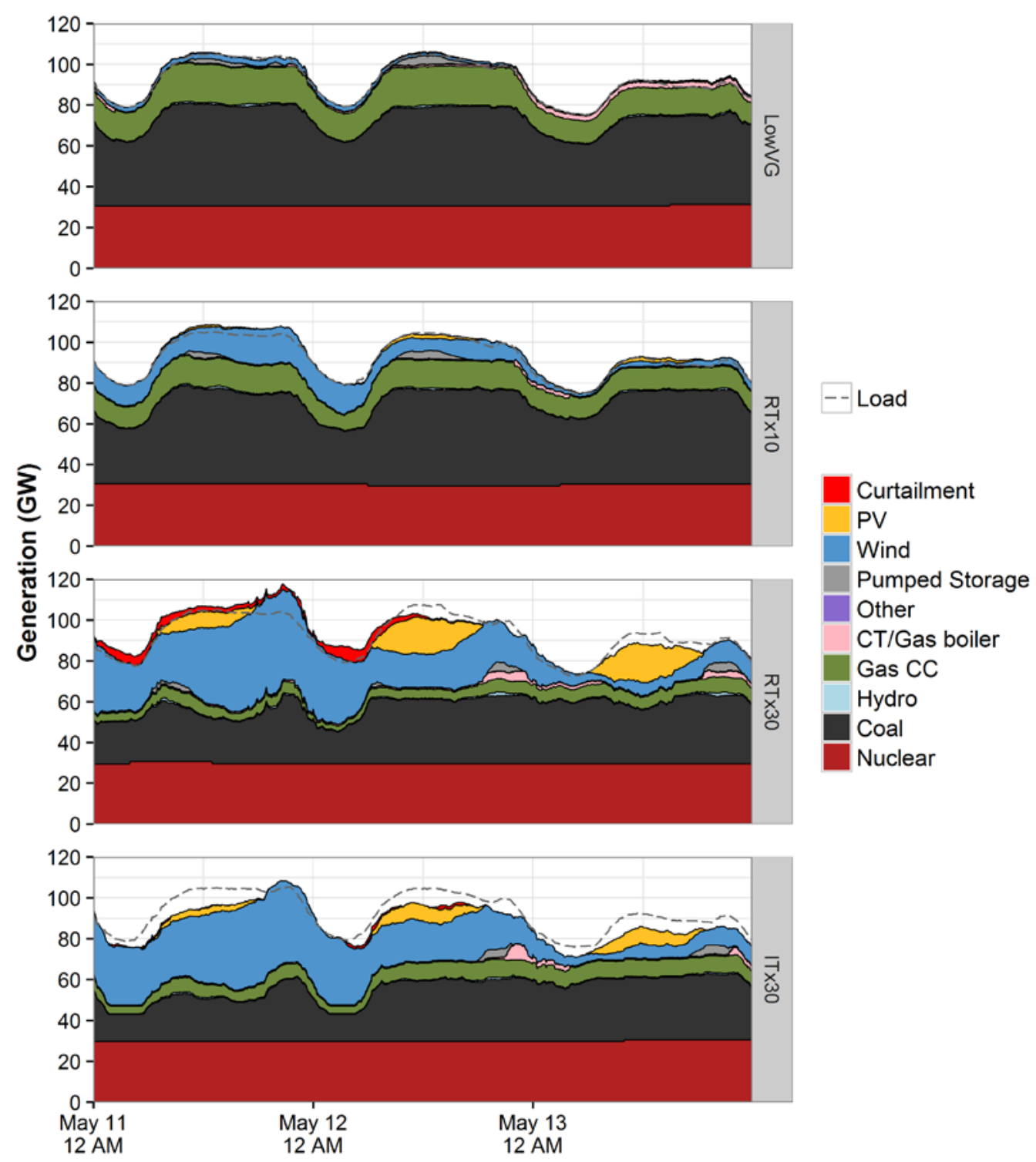

Figure 70. System dispatch in each scenario for PJM during high VG conditions. 


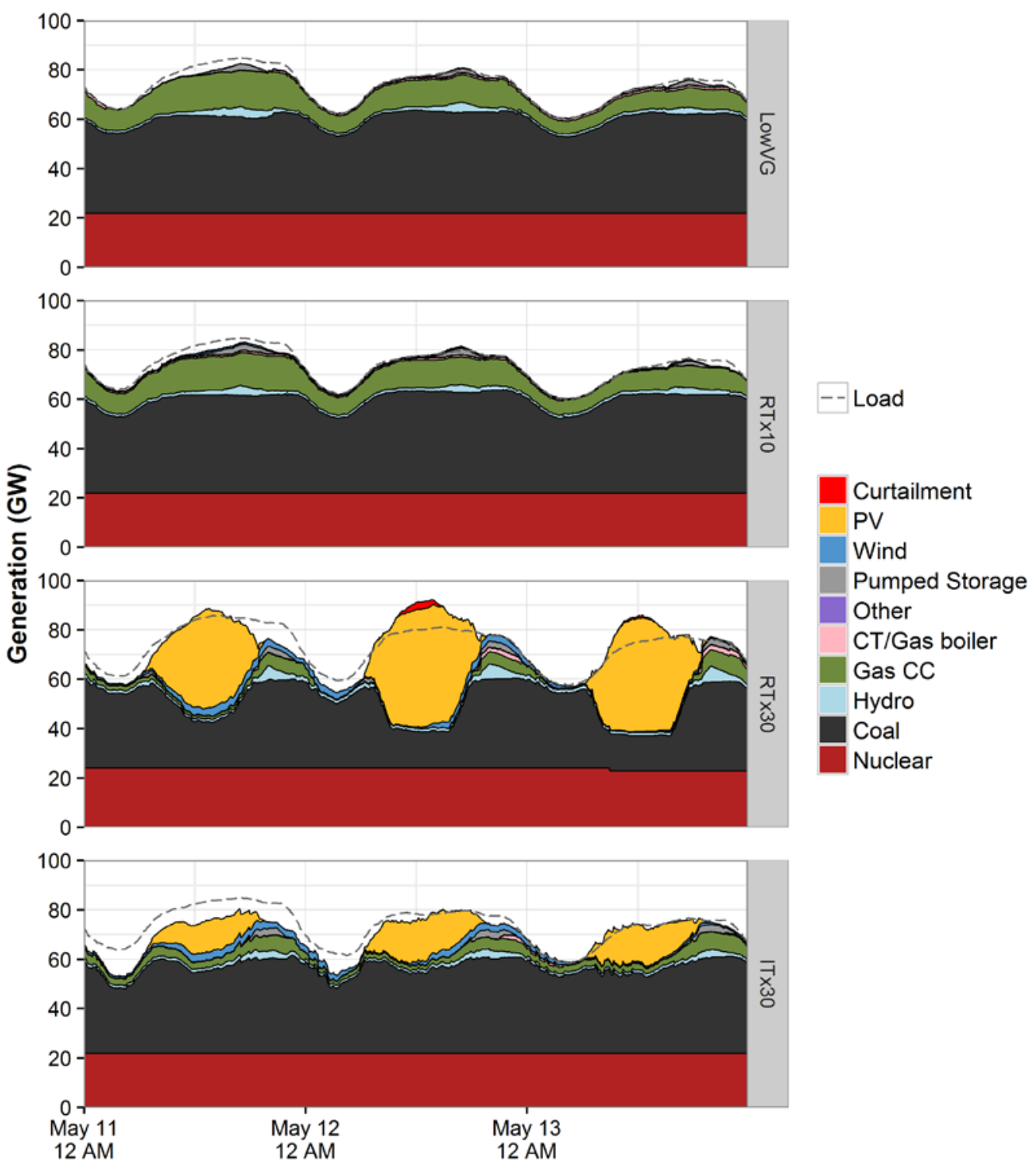

Figure 71. System dispatch in each scenario for SERC during high VG conditions. 


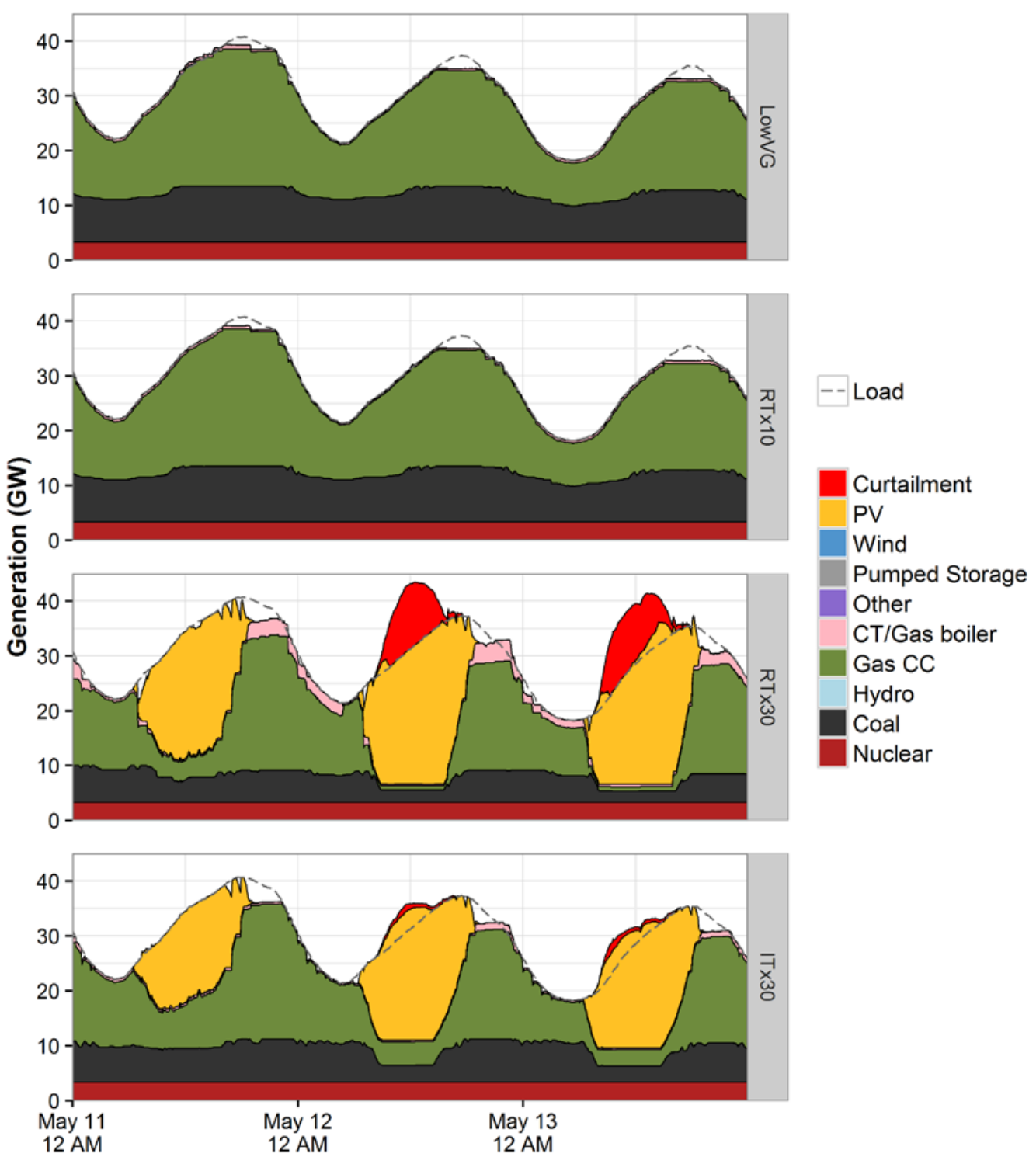

Figure 72. System dispatch in each scenario for FRCC during high VG conditions. 


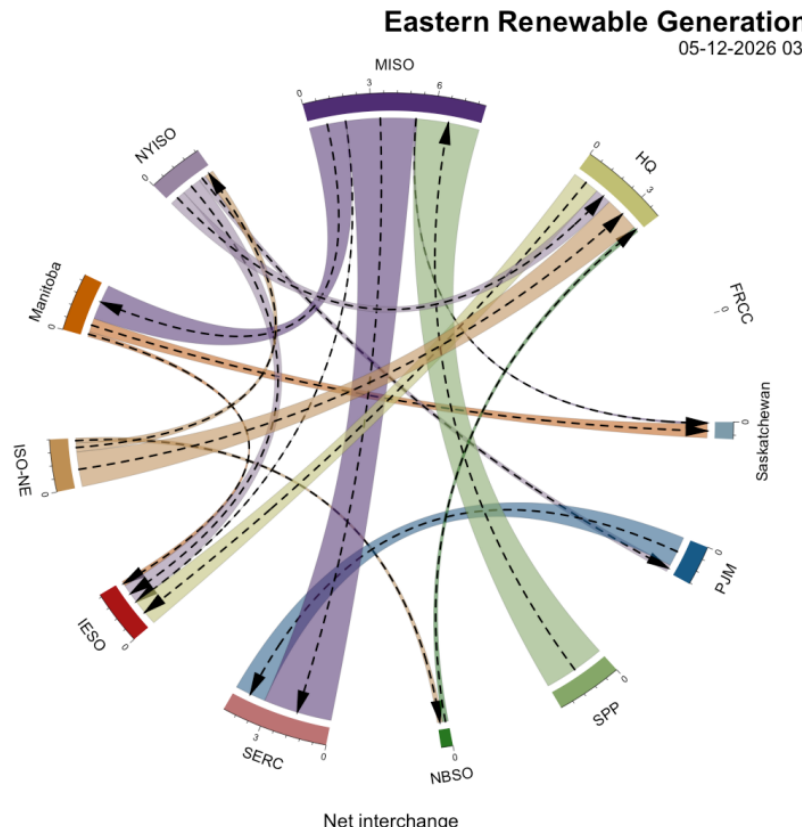

Figure 73. Net interchange in the RTx30 at 03:25 on May 12, 2026

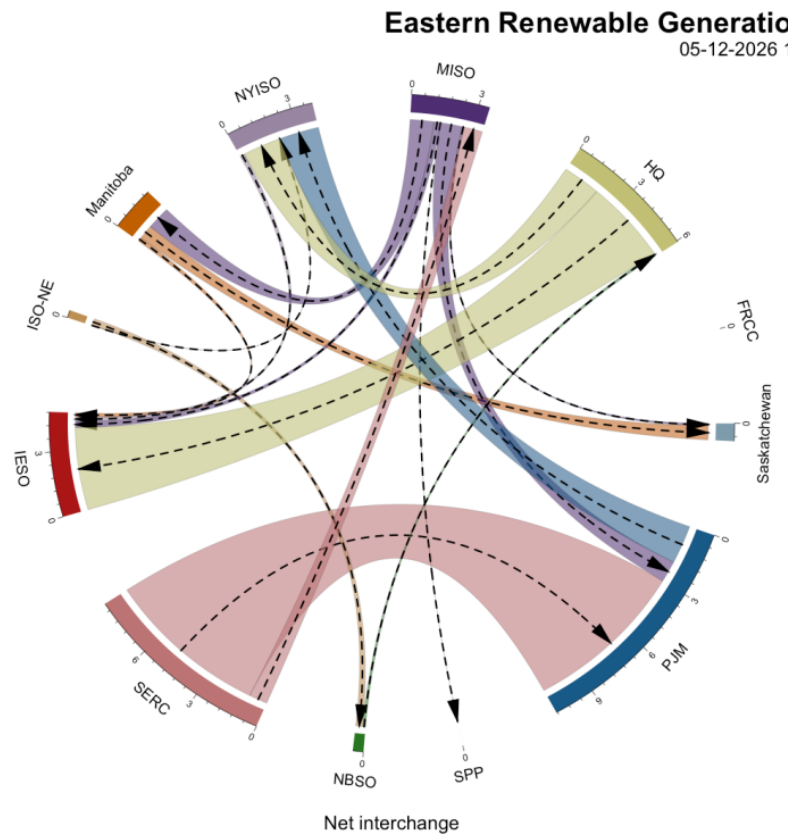

Net interchange

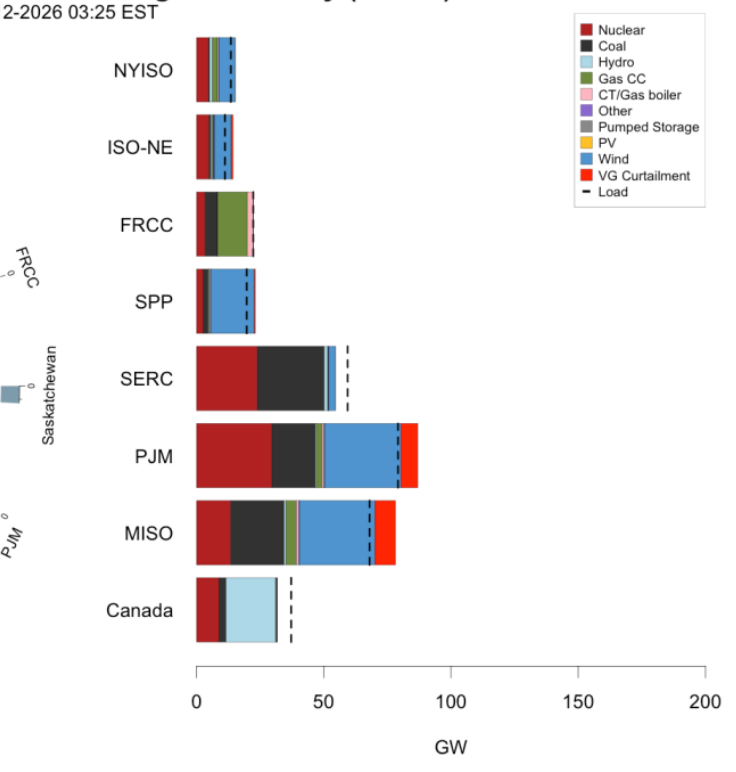

Regional dispatch

Eastern Renewable Generation Integration Study (RTx30)

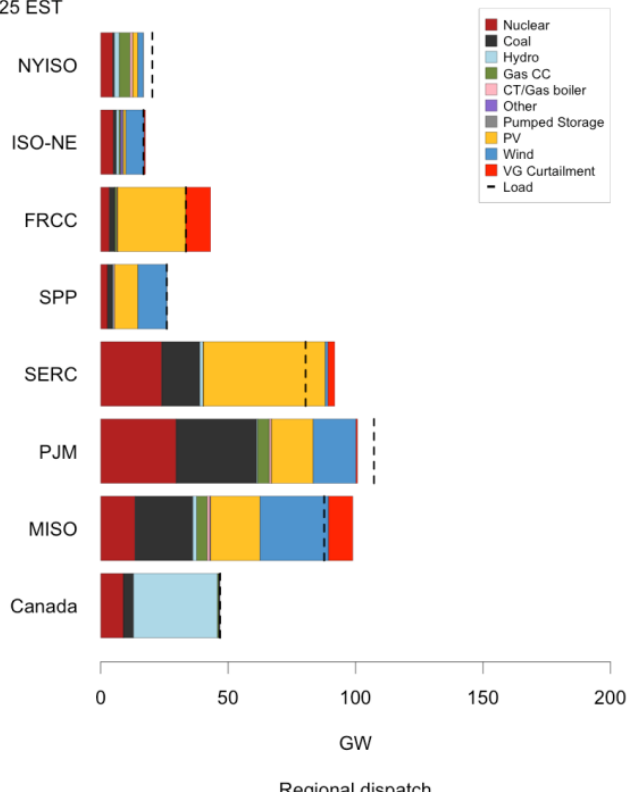

Figure 74. Net interchange in the RTx30 at 13:25 on May 12, 2026 


\subsubsection{U.S. El Highest Net Load Ramp}

Efficient operation of the power system requires operators to commit and dispatch the system under a variety of challenging conditions. We analyzed ten high net load ramps to identify potentially challenging conditions for the EI in the RTx30 and ITx30 scenarios. First, the ten largest positive net load ramps over an eight-hour period were identified for both scenarios. Next, both the absolute VG forecast error for each hour and the total ramp magnitude forecast error were plotted to determine which of the ten net load ramps had the largest forecast error. This analysis indicated the November period had both a very large and quick ramp (i.e. had one of the largest positive net load ramps over an 8-hour period) as well as a large forecast error. We chose to analyze November 23-25 because that period includes three noteworthy events that occur within eight hours:

1. A significant wind forecast error

2. A solar ramp that results from the setting sun

3. A steep increase in load.

Taken together, these three events result in one of the most variable and uncertain periods of operation for the ERGIS simulations. While other periods of operation had high penetration of VG or more extreme differences in the peak and minimum load, this period is noteworthy because of the compounding of these three events.

The dispatch stacks for this time period are presented in Figure 75. In the LowVG we observe low load conditions throughout the period. The lowest load levels in the LowVG case occur after midnight and are similar to low load conditions experienced in the high VG period previously analyzed, i.e. $320 \mathrm{GW}$. In the RTx10 case, high winds in MISO and SPP decrease the net load and result in several hours of VG curtailment in excess of $15 \mathrm{GW}$. As the wind and PV penetration increase in the RTx30 and ITx30, the dispatch stacks for the system change considerably. The minimum net load at 12:00 pm for the RTx30 is $\sim 170 \mathrm{GW}$ while in the ITx30 the minimum net load is $\sim 220 \mathrm{GW}$. We also note significant difference in the slope of the net load ramp between the scenarios.

In Figure 76 we provide the commitment and dispatch for the EI. This figure provides insights into the compounding impact of the wind forecast error, solar ramp, and growing load on the operation of the system. Here we see that the thermal commitment and dispatch for the RTx30 and ITx 30 are very similar even though the amount of wind and PV on the system differs considerably across scenarios. For example, the combined penetration of wind and PV on November 24 at 12:00 PM in the RTx30 is more than $200 \mathrm{GW}$ while in the ITx30 the combined generating capacity for wind and PV is $170 \mathrm{GW}$. Total commitment levels for CC and coal generation are very similar, though the RTx30 experiences deeper cycling of coal and CC plants as a result of daily PV patterns.

In both the RTx30 and ITx30 scenarios system commitment and dispatch are significantly impacted by uncertainty in wind generation. Figure 76 shows that the EI incorrectly forecasts wind generation for both scenarios. On November 23 between sunset and midnight the dayahead forecast is lower than the available wind generation in the real time. Then, shortly after 
midnight on November 24, the system manages a steep unexpected down ramp in wind generation. Figure 77 through Figure 79 show that the forecast errors are similar in their timing and ramp rate in MISO, PJM, and SPP. The magnitude of the forecast error is greatest in the ITx30, as this scenario has a larger amount of wind installed on the system than the RTx30. However, by comparing the net load ramps for the RTx30 and ITx30 in Figure 75 we observe that the steepest net load ramp occurs in the RTx30 on November 24. This difference is driven by the increased presence of PV in the RTx30. The RTx30 has nearly twice as much PV generation on peak. Most of this PV is in SERC and FRCC; however the commitment in nearly every region is impacted by the diurnal pattern caused by PV.

Managing the variable and uncertain conditions presented in this period requires the use of several sources of system flexibility to balance the net load. Committed thermal and hydro resources are redispatched based on operating constraints, large amounts of CTs are committed and dispatched in the real time, and interchange fluctuates rapidly before and after sunrise and sunset to manage regional offsets in VG availability.

We analyzed the differences between scenarios for this period and found the comparative differences in interregional coordination required in the high VG scenarios. Figure 81 shows the dispatch and net interchange for all four scenarios. In the kaleidoscope visualization, interchange is normalized at the maximum interchange for each interval allowing us to compare the differences in transmission utilization across the scenarios. The visualization from this period can be found at www.nrel.gov/ERGIS. Screen shots from two intervals during this period are also show in Figure 80 and Figure 81. As the penetration of VG increases across the scenarios we observe more interchange in the RTx30 and ITx30 scenarios compared to the LowVG and RTx10. The dominant direction of interchange during this period is from the western portion of the system east. However, as the availability of wind unexpectedly decreases and solar generation increases we see a change in the direction of interchange. In Figure 81, we see that SERC is the largest exporter on the system, in both the RTx30 and ITx30 cases. Based on these results, it appears that system operations benefit from multi-regional exports. When wind is bountiful in MISO, SPP and PJM (Figure 80), it is exported to neighboring regions to the north and south. Then during the day, PV generation from SERC in the RTx30 and from SERC and FRCC in the ITx30 is transported to northern neighbors. In some instances PV in the southern United States influences system wide flows all the way to Canada and NYISO. This type of interchange is not observed in the LowVG case, and only appears on occasion in the RTx10, but the direction of flows is dominated by the location and presence of wind. In order to fully understand how wind and PV can impact system wide flows additional analysis is needed to determine whether economic signals and transmission system flexibility can handle large scale changes in direction and magnitude for both expected and unexpected situations. 

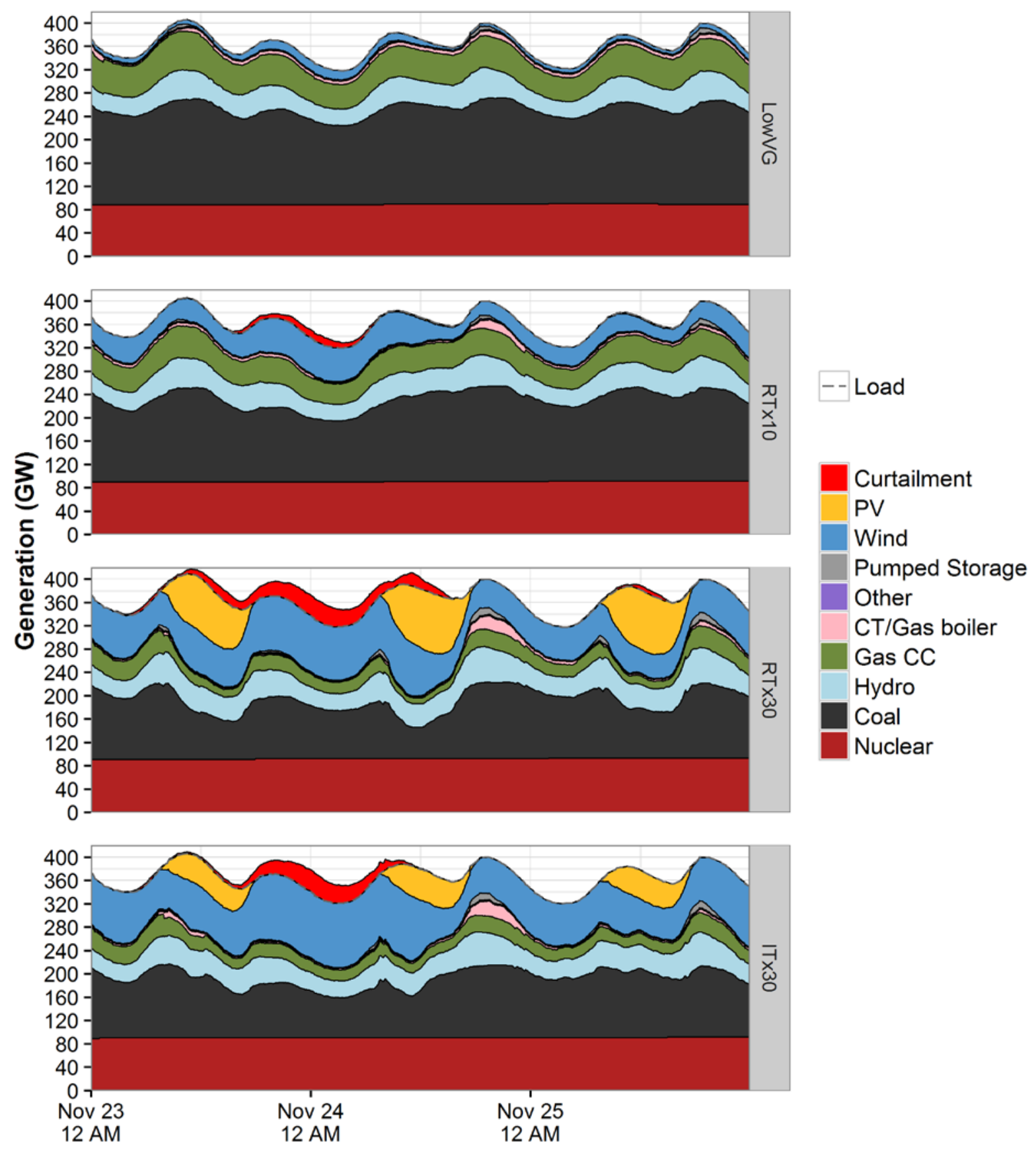

Figure 75. System dispatch in each scenario for the El during high net load ramp conditions 


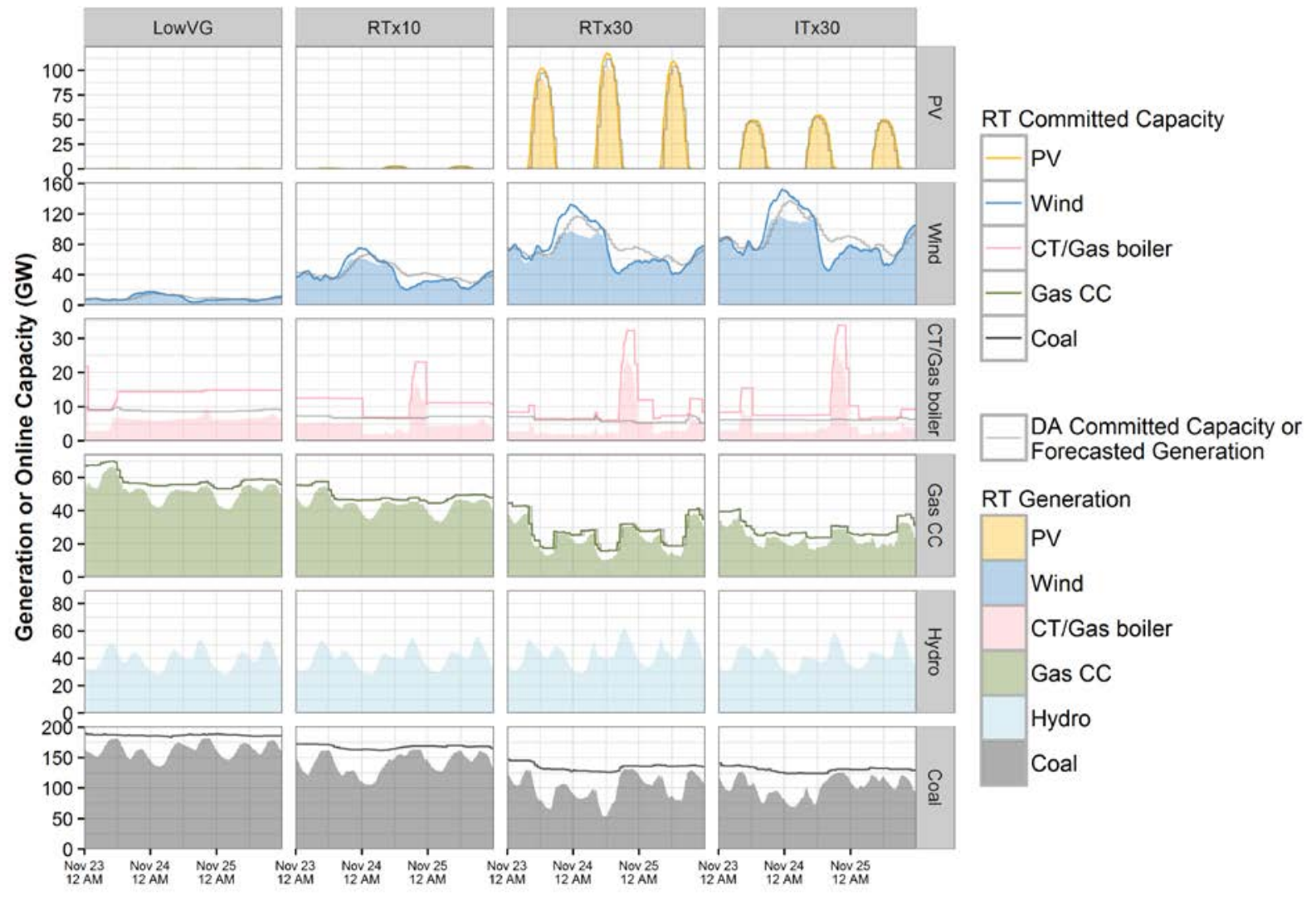

Figure 76. Combined commitment and dispatch of the El for PV, wind, CT/Gas boiler, CC, hydro, and coal during high net load ramp conditions

For a guide to interpreting this plot, please refer to Figure 57. 




Figure 77. Combined commitment and dispatch of the MISO for PV, wind, CT/Gas boiler, CC, hydro, and coal during high net load ramp conditions

For a guide to interpreting this plot, please refer to Figure 57. 


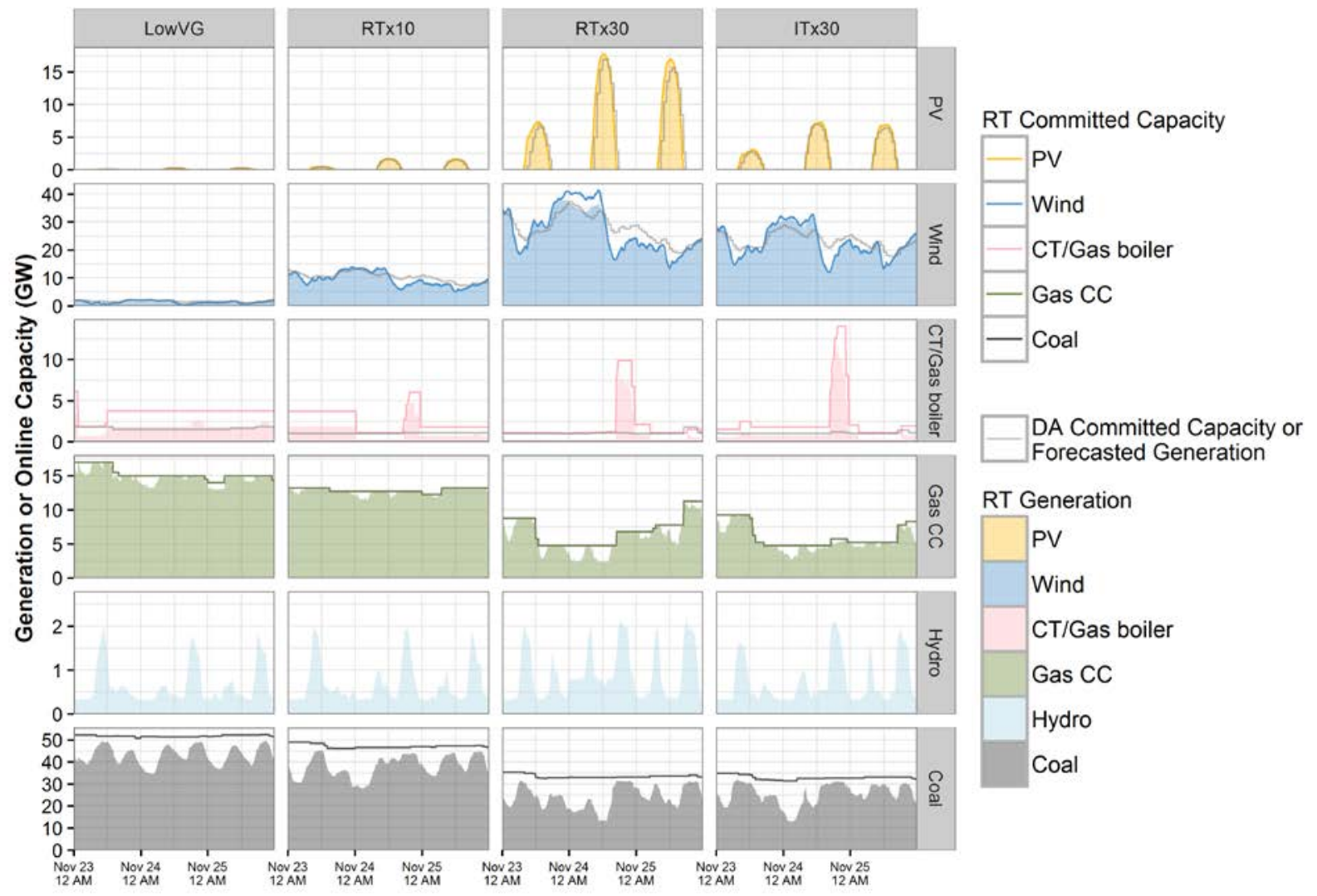

Figure 78. Combined commitment and dispatch of the PJM for PV, wind, CT/Gas boiler, CC, hydro, and coal during high net load ramp conditions

For a guide to interpreting this plot, please refer to Figure 56. 


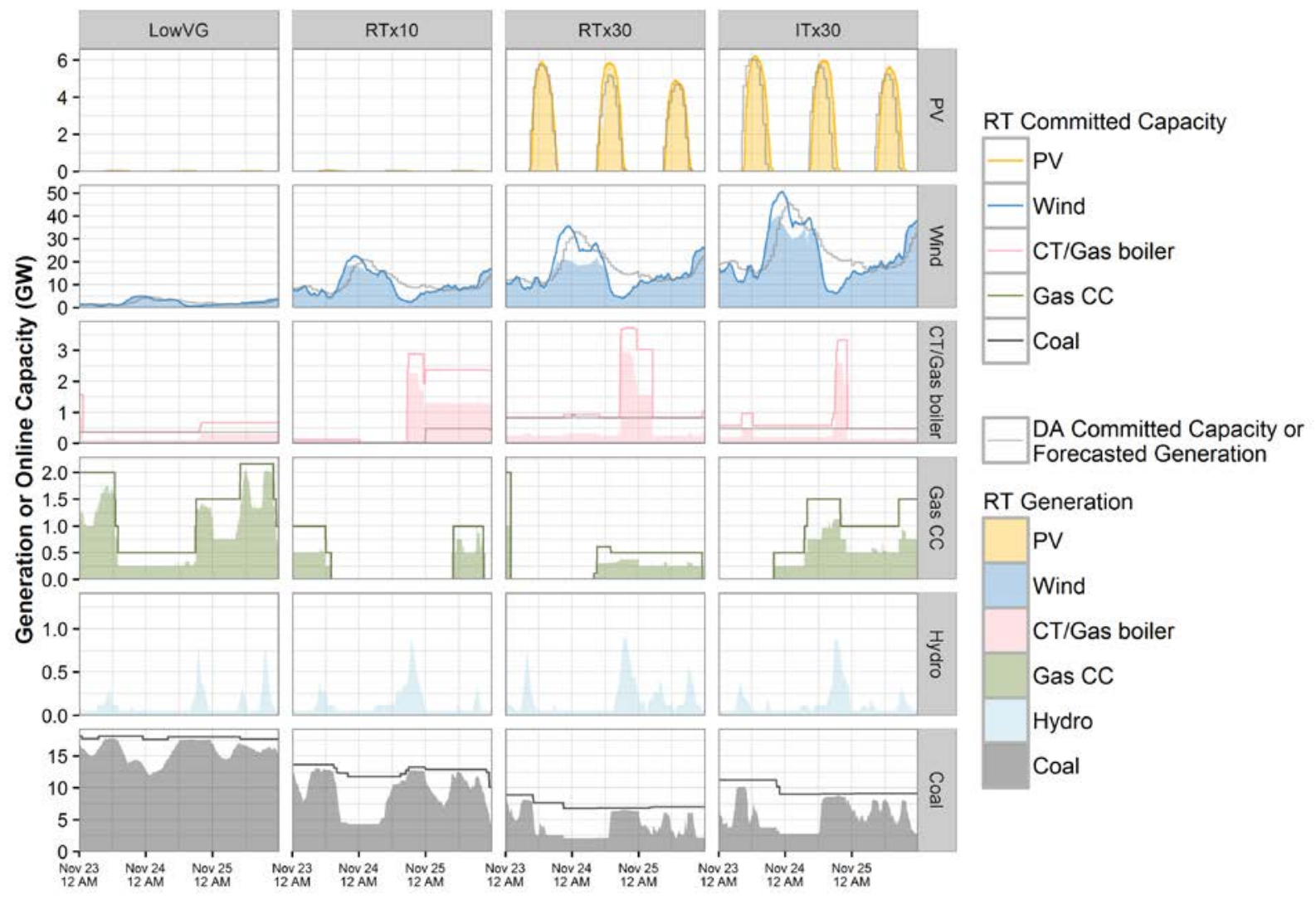

Figure 79. Combined commitment and dispatch of the SPP for PV, wind, CT/Gas boiler, CC, hydro, and coal during high net load ramp conditions

For a guide to interpreting this plot, please refer to Figure 56. 

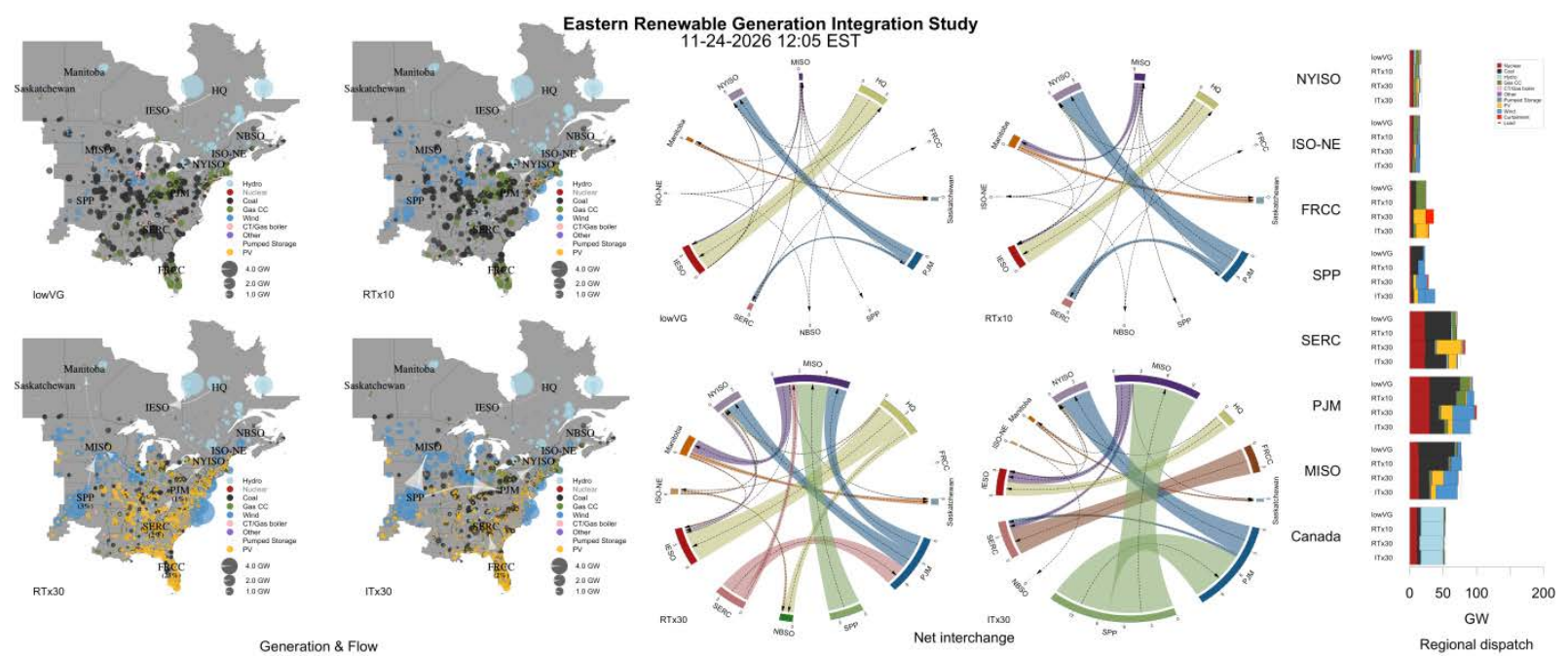

Figure 80. System dispatch and net interchange for the El in all 4 scenarios at 12:00 November 24, 2026

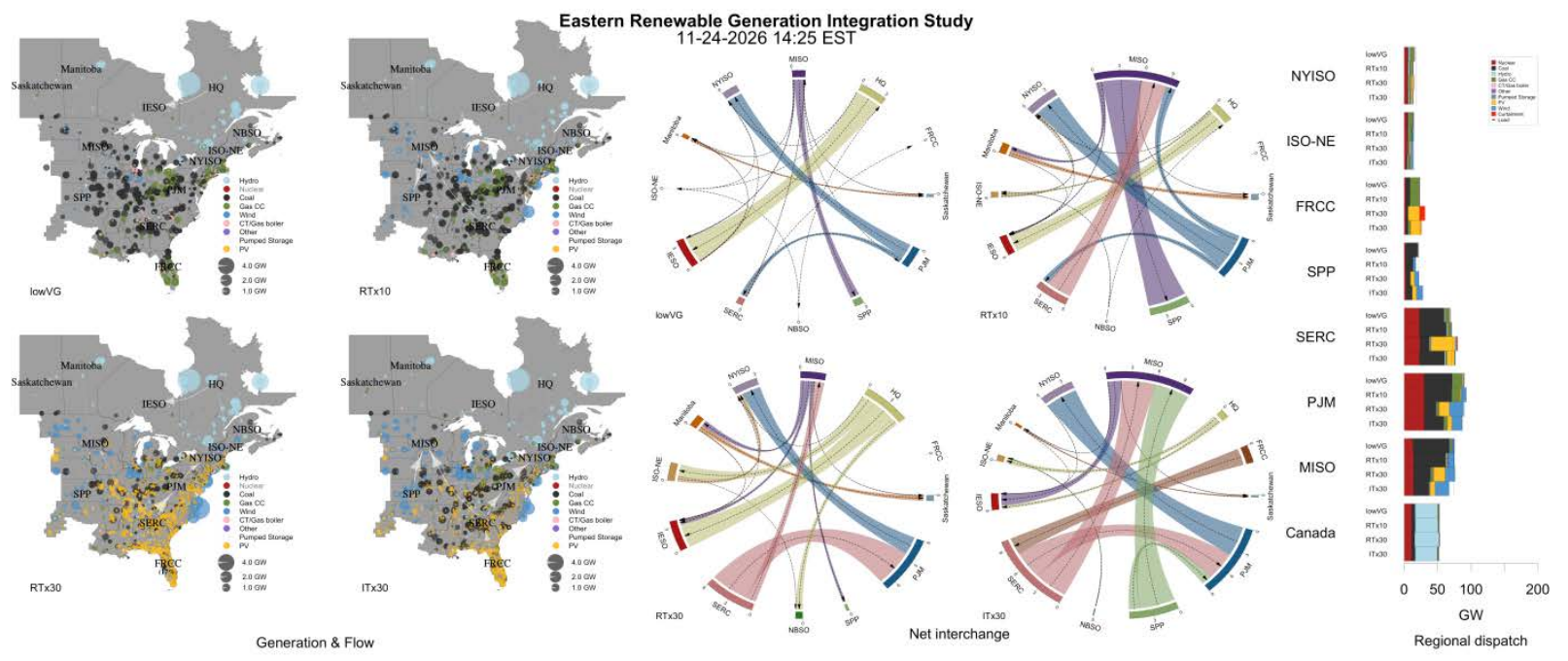

Figure 81. System dispatch and net interchange for the El in all 4 scenarios at 14:25 on November 24, 2026 


\subsection{Operational Impacts}

\subsubsection{Impacts on Thermal Generation}

A large penetration of VG necessitates greater flexibility than is typically utilized in a power system made up of only thermal and hydro generators. A number of integration studies have shown that much of this flexibility can be found in operational changes to these traditional generators, such as increased starts and more part-load operation (EnerNex 2011, GE Energy 2010, Lew 2013, GE Energy Consulting 2014a, GE Energy Consulting 2014b, CAISO 2010) , as well as grid operating reforms and well-designed ancillary services markets. This section summarizes impacts of operational changes on coal, CCs, and CT/Gas boiler for the U.S. EI, and includes analysis of how inter-regional resource diversity affects these operational impacts.

\subsubsection{Reduced Commitment and Generation}

Figure 83 shows the impact of VG on the overall usage of thermal generators. The figure presents U.S. EI-wide results. The figure shows the fleet-wide average capacity factor (top), output level when committed (middle), and percent of time at minimum generation (bottom), for each generator type and scenario. As more VG is added to the system, the capacity factors of traditional generators decrease (Figure 83, top). ${ }^{35}$ The decrease due to displacement of traditional generation by VG. A $30 \%$ increase in VG leads to about a $30 \%$ decrease in the capacity factors for coal and about $45 \%$ for CCs. Further analysis is necessary to understand the financial impacts of low capacity factors.

The results of generator output for committed units are shown in Figure 83, middle. Average capacity factor is calculated as the total energy produced divided by the total nameplate capacity of the generators and the amount of time in the year. The capacity factor includes outages, decommitments, and part-load operation. The decrease in output from committed units as VG increases is less pronounced, and in some cases non-existent. While committed, coal units see mild decreases in output, and CC shows similar but more modest changes. The decrease in output level when committed indicates that coal and CC generators are operating in a loadfollowing (or net-load-following) mode more often with increased VG. Output level changes more in RTx30 than in other scenarios, suggesting that balancing of regional resources in combination with more $\mathrm{PV}$ requires greater flexibility from the online baseload generators than does the ITx 30 .

Figure 83 also shows the percent of time the individual units spend at their minimum stable generation level when they are online (see Section 4.1.1 for details of minimum stable levels). All three generator types increase the fraction of time they operate at their minimum generation level in the high VG scenarios. The RTx30 has the largest increase in the fraction of time spent at minimum generation level for the coal and CC units. SPP and MISO, the areas with the most VG capacity in ITx 30 , have relatively small amounts of CC generation, especially as CCs are displaced in the higher VG scenarios. Therefore, CT/Gas boiler units are used, along with coal, to follow load in these regions. CT/Gas boilers see their biggest differences in the ITx30.

\footnotetext{
${ }^{35}$ Average capacity factor is calculated as the total energy produced divided by the total nameplate capacity of the generators and the amount of time in the year. The capacity factor includes outages, decommitments, and part-load operation.
} 
The impact on thermal plant capacity factors is impacted by the fact that the same thermal plant fleet was used in all scenarios. Capacity factor is calculated as actual output divided by maximum potential output. By keeping the thermal fleet constant, we likely included a large number of thermal plants that would not be needed and would likely no longer operate in a real system with our simulated wind and PV penetrations. This may increase the capacity factor of the units that do remain in the market. 

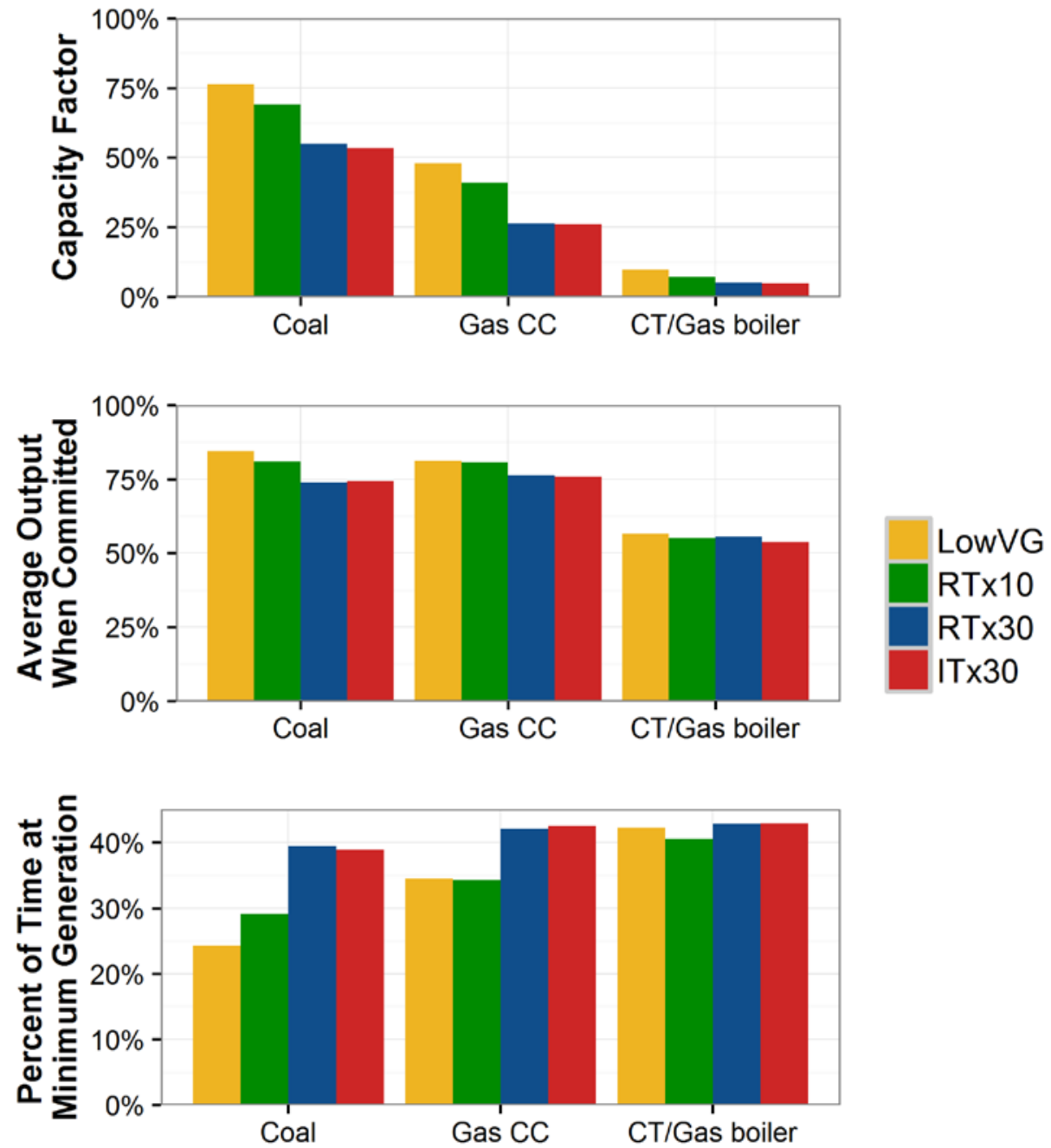

Figure 82. Capacity factors of thermal units for the total fleet in the U.S. El (top), Average output of committed units (middle) and percent of online time at minimum generation (bottom)

Capacity factors are calculated as the nameplate capacity of the generators by fuel type for all periods of the year divided by the total energy produced. 'Average output when committed' counts only committed units and calculates the output as a percentage of nameplate capacity of the unit.

\subsubsection{Ramping and Starting}

Figure 86 shows the total number of positive ramps normalized by the amount of energy generated by generator type ${ }^{36}$ for the U.S. EI as well as each U.S. region. As expected, coal ramps less than the more-expensive and more-flexible CCs and CT/Gas boilers. Coal and CT/Gas boilers see impacts even in the RTx 10, and the higher penetration RTx30 and ITx30 increase ramps further. RTx30 has the greatest impact for all fuel types system-wide, particularly the $\mathrm{CT} / \mathrm{Gas}$ boilers.

\footnotetext{
${ }^{36} \mathrm{~A}$ ramp is counted when a unit changes its power output in the positive direction more than $30 \%$. Only positive ramps are counted in this calculation.
} 
Also shown are the ramps per quantity of energy generated for each of the U.S. EI regions (Figure 85). The impact on CT/Gas boilers is very different in the different regions, and high PV regions such as FRCC and SERC drive the trend shown in the EI-wide figure. The behavior in FRCC and SERC is caused by an evening decrease in PV output while demand remains high. CCs provide a large part of the required ramp up in thermal power output, which is also reflected in these regions for $\mathrm{RT} \times 30$, but $\mathrm{CT} / \mathrm{Gas}$ boilers play a key part in providing energy and ramping for a short amount of time before the load begins decreasing in the late evening.

The trends in coal are indicative of its need to follow net load more often in scenarios with high VG penetration. As gas is displaced, coal is on the margin more often and therefore expected to follow changes in net load. This is especially pronounced in the RTx30 case because of the increased daily cycling due to the greater installed PV capacity. CCs follow similar trends in that, U.S. EI-wide, the impact of ramping is most pronounced in the RTx30 case. Most individual regions also follow a similar pattern. 

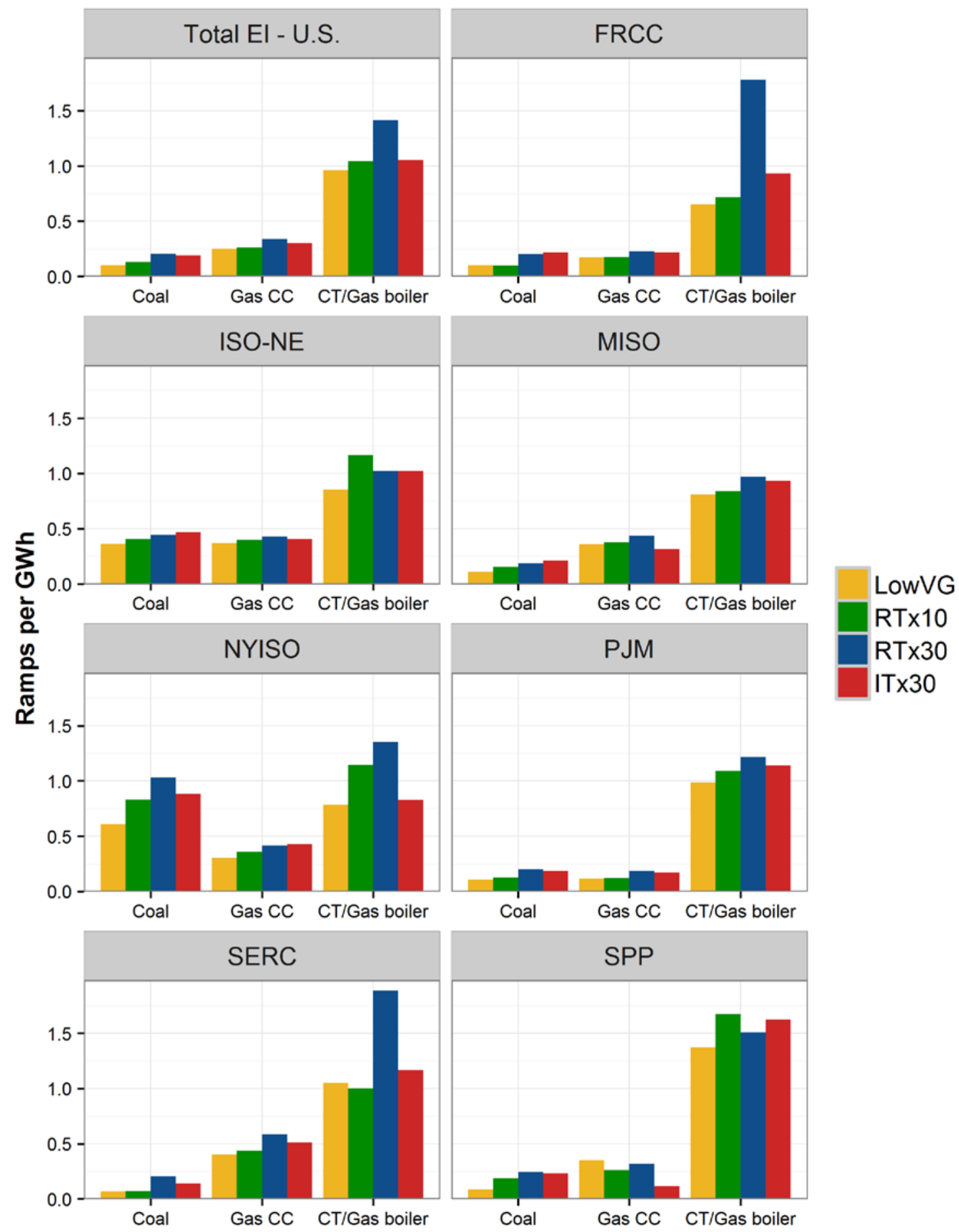

Figure 83. Number of ramps for the total U.S. El and U.S. regional fleets divided by the energy produced

Ramps are counted as a unit changing its output in the positive direction for more than $30 \%$ over any time period. 
Committing and decommitting thermal units is another form of flexibility within the power system. Figure 87 shows that as VG penetration increases, the capacity-starts ${ }^{37}$ increase for coal and $\mathrm{CC}$ units and decrease for $\mathrm{CT} / \mathrm{Gas}$ boiler units. The increase in $\mathrm{CC}$ and $\mathrm{CT} / \mathrm{Gas}$ boiler capacity-starts is most pronounced in the RTx30. The RTx30 also shows more capacity starts than the ITx30, for two primary reasons. First, there is about twice as much PV throughout the U.S. EI in the RTx30 as in the ITx 30 . The larger amount of PV results in larger and more frequent daily changes in net load and makes $\mathrm{CC}$ units (which have shorter minimum up and down times) cycle more often. Second, the increased interregional transmission in the ITx30 allows greater use of imports and exports for balancing net load.

Figure 87 also shows the capacity started and average number of days online per start by generator type and scenario. All three generator categories experience decreases in the average number of days online per start as the VG penetration increases. Coal generators decrease from about 37 days in the LowVG to 28 in the RTx10, and 20 in the RTx30 and ITx30. CC generators decrease from about six days down to about two days in the RTx30 and ITx30. CT/Gas boiler generators have average runtimes of less than one day in all four scenarios.

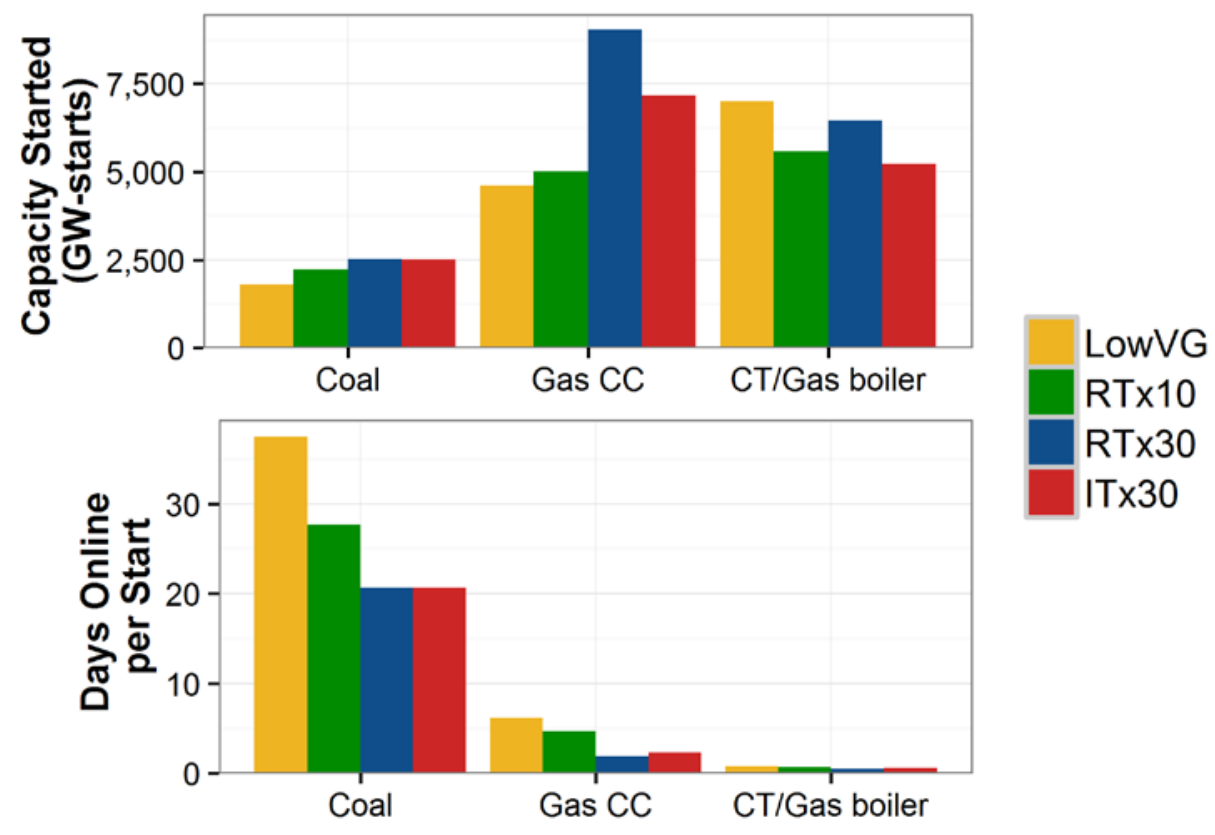

Figure 84. Capacity-starts (capacity in GW multiplied by number of starts, for each unit) for the U.S. El generators by fuel type (top), and the average number of days online per start (bottom)

\footnotetext{
${ }^{37}$ The capacity-starts metric is the capacity-weighted number of starts per year. Capacity-starts is calculated as the sum of each unit started multiplied its capacity. Capacity-starts is used instead of starts to capture the impact of generator size.
} 


\subsubsection{Generation from Hydro and Pumped Storage}

Analysis of average daily hydro generation and pumped hydro profiles illuminates how changes in wind and PV penetration might impact hydro generation and pumped storage operation. Figure 88 shows the average daily generation for hydro generation in all study regions. The highest amounts of hydro generation in the United States are located in SERC, NYISO, and MISO. ${ }^{38}$

Despite the substantial increase in wind generation from the LowVG to the RTx10, we observe very little change in average daily hydro operation, although MISO does have some small changes between the scenarios. In all regions and scenarios, hydro generation ramps up with the morning load and peaks during historical peak load periods. However, in the ITx30, which has a $5 \% \mathrm{PV}$ penetration, we see the emergence of a more pronounced dual peaking pattern in hydro plant generation. This distribution mirrors average diurnal patterns discussed in section 4.2.2. The dual peaking pattern in hydro generation increases in the RTx30 scenario, where PV supplies $10 \%$ of all U.S. EI load. The PV generation in the daylight hours pushes the optimal use of the hydro generation away from the mid-day hours and toward the morning load ramp and the evening peak. Compared to all other scenarios, the average daily hydro profiles for the RTx30 case are both greater, from a GW perspective, and shifted farther from the mid-day hours. As described in Section 4.1.1, hydro generators were constrained to have a minimum generation level of $20 \%$ of their maximum capacity during all hours to represent non-power-production constraints.

\footnotetext{
${ }^{38}$ Nameplate installed capacities in ERGIS model: 11 GW in SERC, 5 GW in NYISO, and 5 GW in MISO.
} 


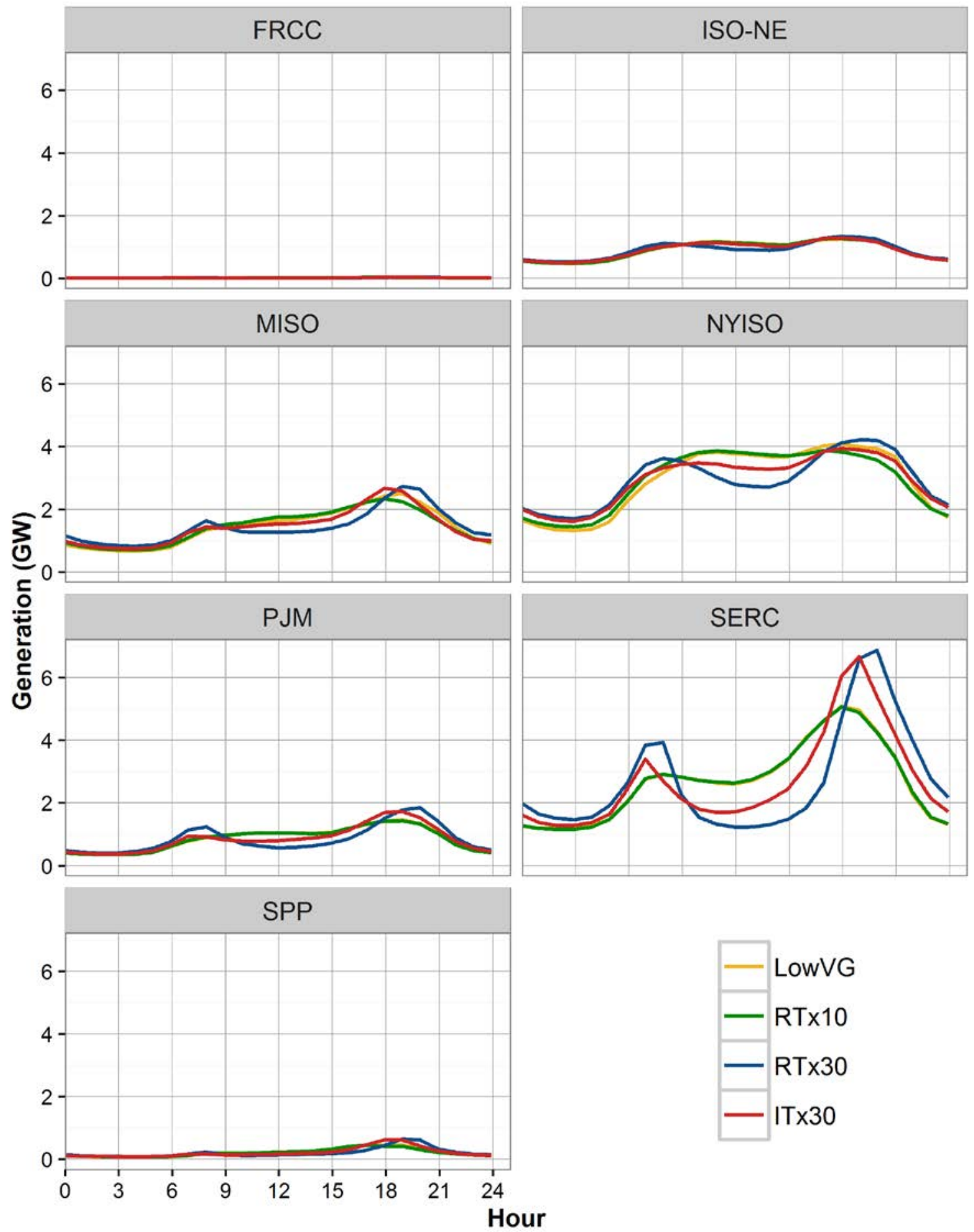

Figure 85. Average daily hydro generation in all scenarios

Pumped hydro facilities see a substantial shift in operations under high penetrations of wind and PV (Figure 89). As with traditional hydro, MISO is the only region with substantial differences in pumped hydro operation in the RTx10, as compared to the LowVG. There are considerable changes in the timing and peaks of pumped hydro generation as the VG penetration increases in 
the RTx30 and ITx30. These peaks are most significant in the RTx30. Morning load and PV peaks appear to have the same general shape in all regions and scenarios. However, in the RTx30 the pumped hydro generation is pushed further away from the mid-day hours. There are significant periods of time during the middle of the day where pumped hydro resources do not generate any electricity, which is a departure from operations in the LowVG and RTx10 cases.

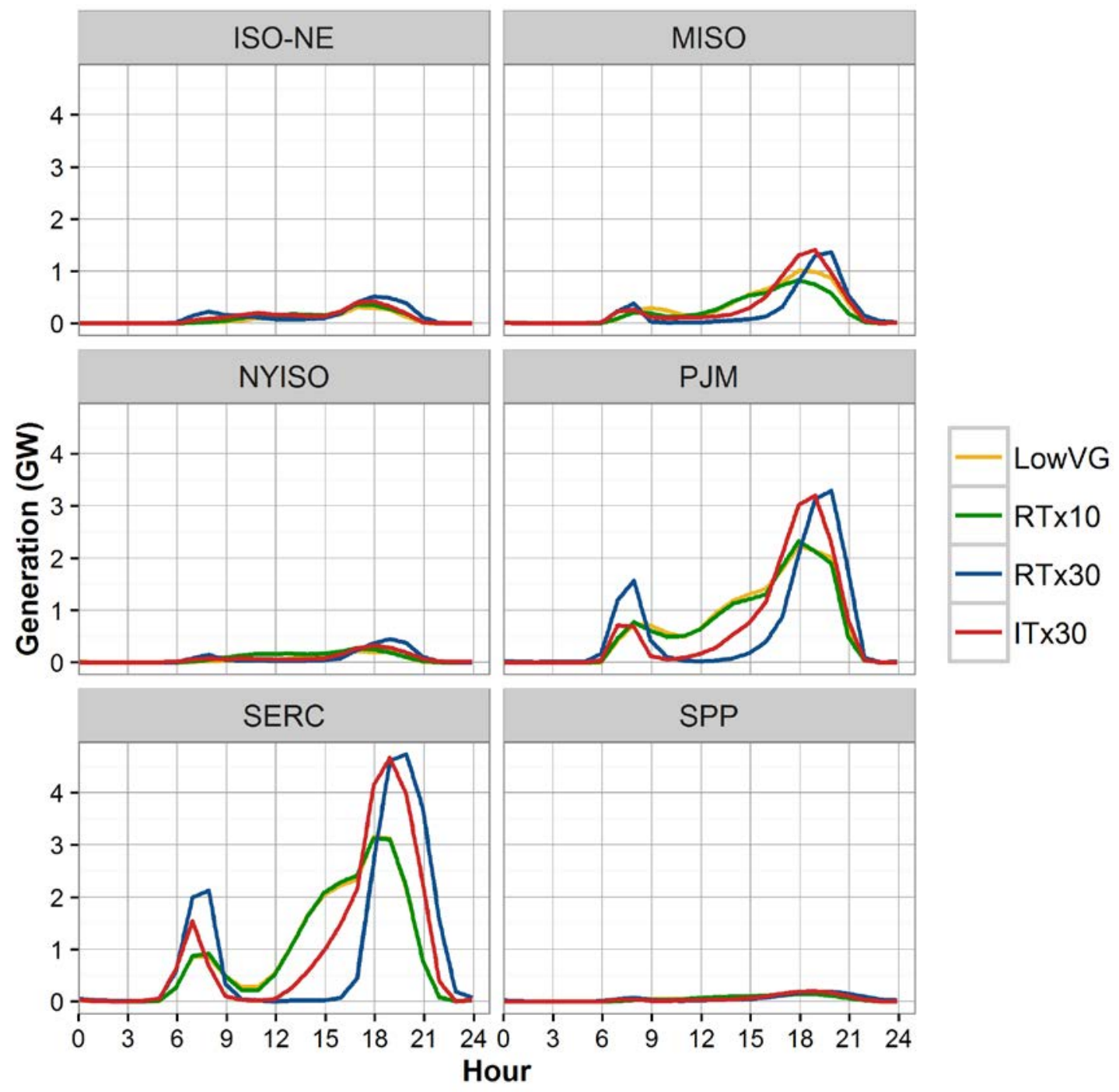

Figure 86. Average daily pumped hydro operation in all scenarios

On an average basis, hydro resources and pumped storage in the US contribute to balancing the diurnal variability of PV resources. As PV generation increases in FRCC and SERC, hydro generation and pumped storage operations in neighboring regions change. The interrelationship of hydro plant operations in the east and PV generation is an area that merits additional research. In contrast, diurnal patterns in wind generation are weaker and do not result in visible changes in daily hydro operations. Additional analysis of the impact of VG expansion in the eastern US on hydro operations in the Canadian provinces could be conducted in future work to understand broader impacts on hydro and pumped storage resources. 


\subsubsection{Wind and PV Curtailment}

VG curtailment is a reduction in the output of a wind or PV generator from what it could otherwise produce for given meteorological conditions. There can be a variety of compounding causes of wind and PV generation curtailment, including VG generator type and placement, available transmission capacity, thermal plant operating limits (primarily minimum stable operating levels), and reserve requirements.

Table 49 shows how much VG is curtailed as a percentage of the available energy, and Figure 90 shows the total available VG energy that is curtailed in each of the study scenarios. VG curtailment is not specifically assigned to wind or PV because they have the same marginal cost of zero. As described in Sections 4.3.2, 4.3.3, and 4.3.5, transmission expansion and VG capacity and siting are different between each scenario. Both of these assumptions drive differences in the curtailment results. The RTx30 has significantly higher VG curtailment than the other scenarios, however direct comparisons of the different VG curtailment levels are inappropriate because of the underlying differences in the scenarios' assumptions. This is because of differences in the transmission expansions and installed wind and PV capacities and locations.

Table 49. Available Wind and PV Curtailed

\begin{tabular}{|l|r|}
\hline Scenario & VG Curtailment (\%) \\
\hline LowVG & 0.1 \\
\hline RTx10 & 2.7 \\
\hline RTx30 & 6.2 \\
\hline ITx30 & 3.8 \\
\hline
\end{tabular}

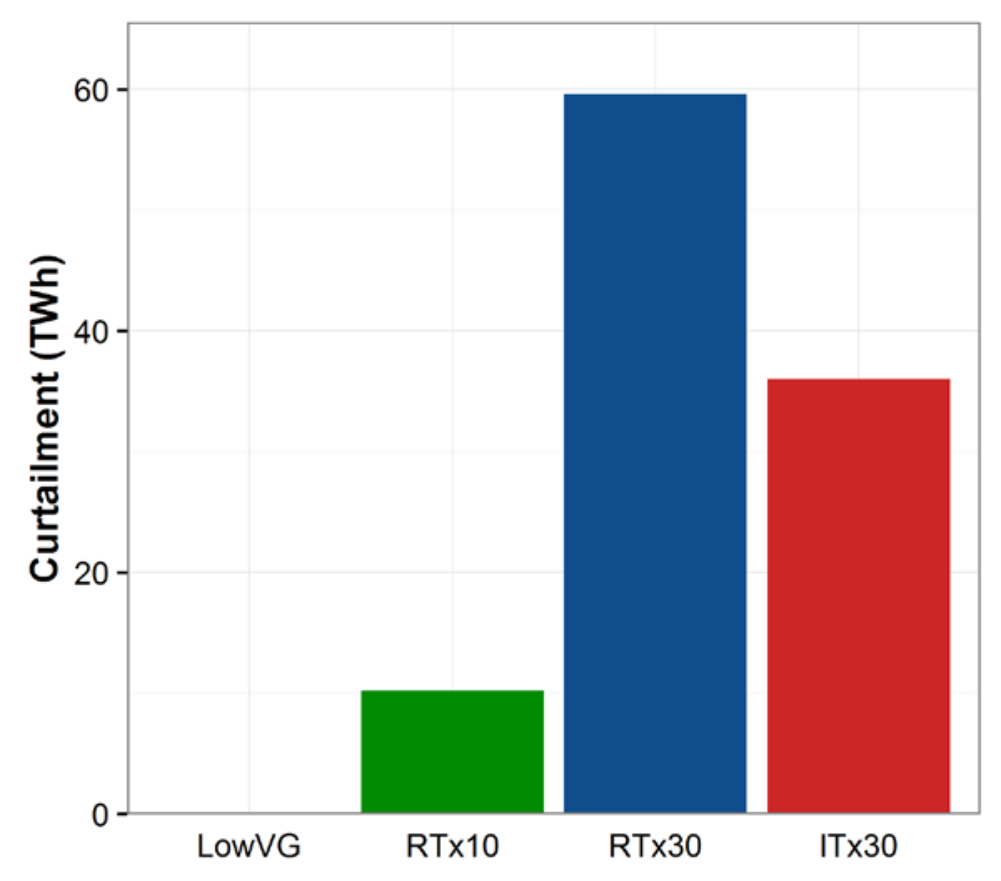

Figure 87. Curtailment of available wind and PV generation 
A duration curve of wind and PV curtailment (Figure 91) shows that the maximum hourly VG curtailment in the RTx30 is nearly $70 \mathrm{GW}$, and VG curtailment exceeds $40 \mathrm{GW}$ during numerous 5 -minute intervals. Most of the periods above $40 \mathrm{GW}$ are in March and April and are confined to 13 individual days. Maximum hourly VG curtailment in the ITx30 and RTx10 cases are lower, with peaks of approximately $40 \mathrm{GW}$ and $18 \mathrm{GW}$, respectively.

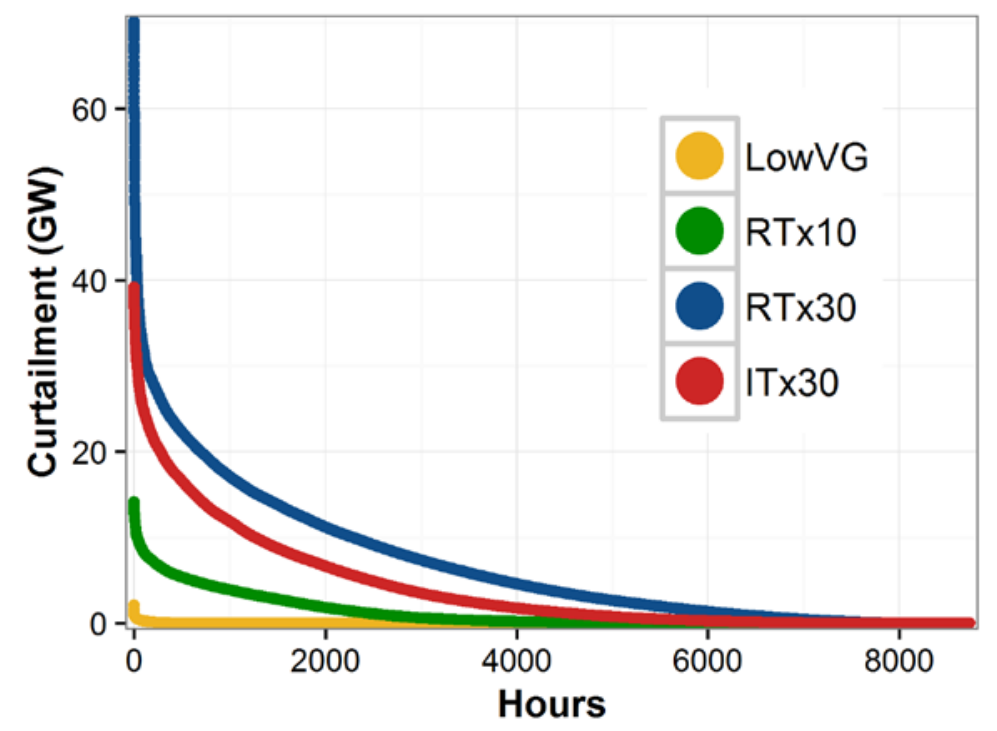

Figure 88. U.S. El duration curve for wind and PV curtailment

VG curtailment in all of the scenarios follows seasonal trends in wind and PV generation (Figure 92), which is anti-correlated to seasonal electricity demand. Peak load for the U.S. EI occurs in early August. The most VG curtailment, in all scenarios, occurs in the months of March and April; however VG curtailment in the RTx30 is much higher than the ITx30.

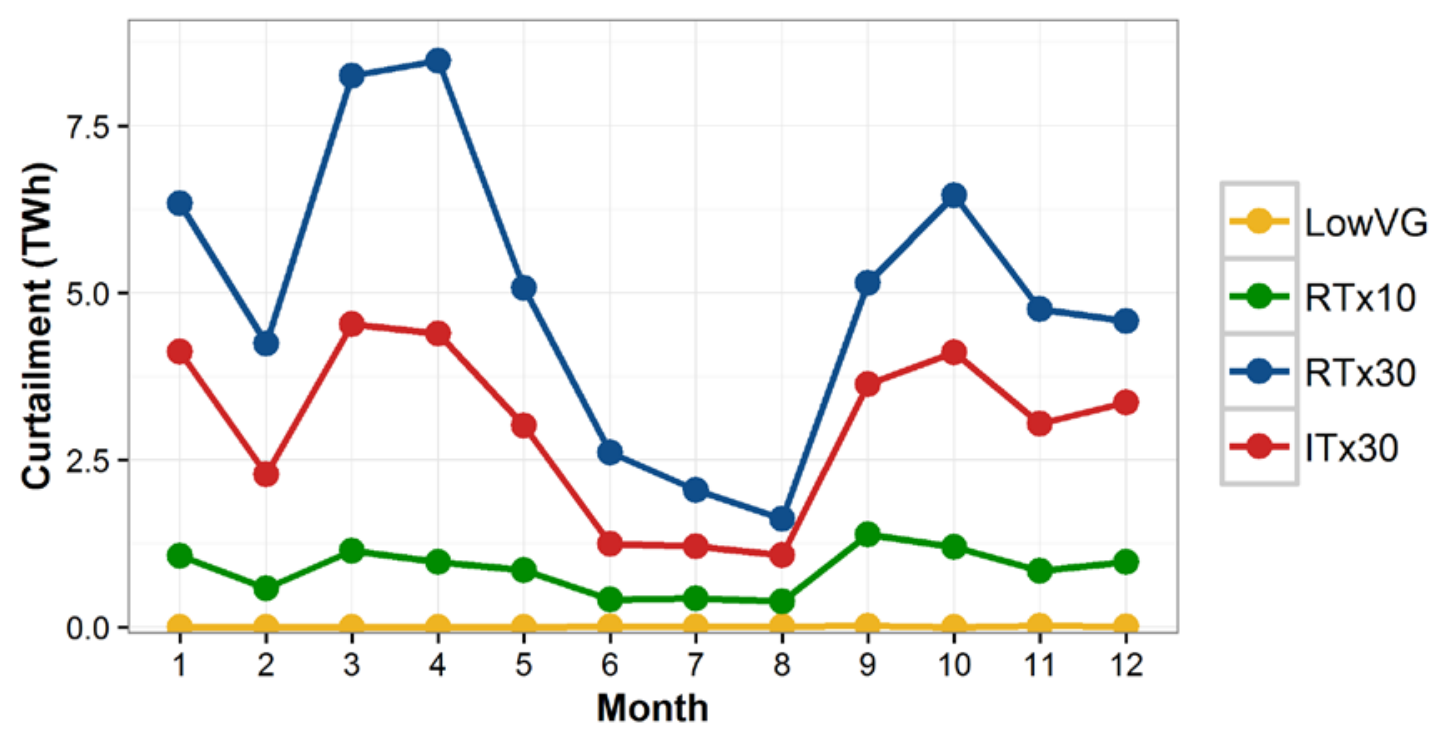

Figure 89. Monthly VG curtailment profiles for each scenario 


\subsubsection{Regional VG Curtailment Analysis}

Figure 93 shows the annual average daily profile of VG curtailment for the whole U.S. EI and for each U.S. EI region. For the whole EI, and most of the regions, the VG curtailment is highest in the RTx30 scenario, and a clear daily pattern of high VG curtailment at midday is visible. The ITx30 also shows significant VG curtailment and a similar pattern at night although lower VG curtailment during the day.

The U.S. EI daily pattern in the RTx30 shows two peaks. The first peak occurs in the early morning before load increases. This VG curtailment is entirely wind curtailment, as there is no PV generation on the system at this time. The VG curtailment decreases rapidly as the load picks up in the morning. A second peak occurs at mid-day as PV curtailment increases and peaks, and then curtailment decreases as PV generation decreases in the afternoon and the load generally remains high.

In contrast to the RTx30, the U.S. EI daily pattern in the ITx30 shows a single peak during the low-load early morning hours. VG curtailment decreases over the course of the day as load increases and remains high, and then begins increasing again when load begins decreasing around 21:00.

The daily pattern in the RTx10 follows a similar trend to the ITx30, as the VG in both is dominated by wind generation. The RTx10 has lower overall VG curtailment levels and a narrower peak than the ITx30.

Comparison of the RTx30 and ITx30 indicates that part of the VG curtailment can be attributed to the different transmission expansions used in the two scenarios. In the nighttime hours, PV generation is unavailable and the only resource that can be curtailed is wind. The difference in nighttime VG curtailment is primarily from SPP, where the RTx30 has higher VG curtailment than the ITx30 despite the fact that the RTx30 has lower installed wind capacity. The difference is the larger ITx30 transmission expansion, which allows more export to other regions.

A different component of the VG curtailment can be attributed to PV. FRCC shows significant VG curtailment in both the RTx30 and ITx30 scenarios. FRCC does not have any installed wind capacity, so all of the VG curtailment is PV. The RTx30 and ITx30 transmission expansions have the same amount of export capacity between FRCC and SERC, so the difference between the two is mainly due to the VG in the two scenarios. PV generation from FRCC and SERC is generally coincidental. This makes it particularly challenging for FRCC to export excess PV generation to SERC, thus increasing PV curtailment. 


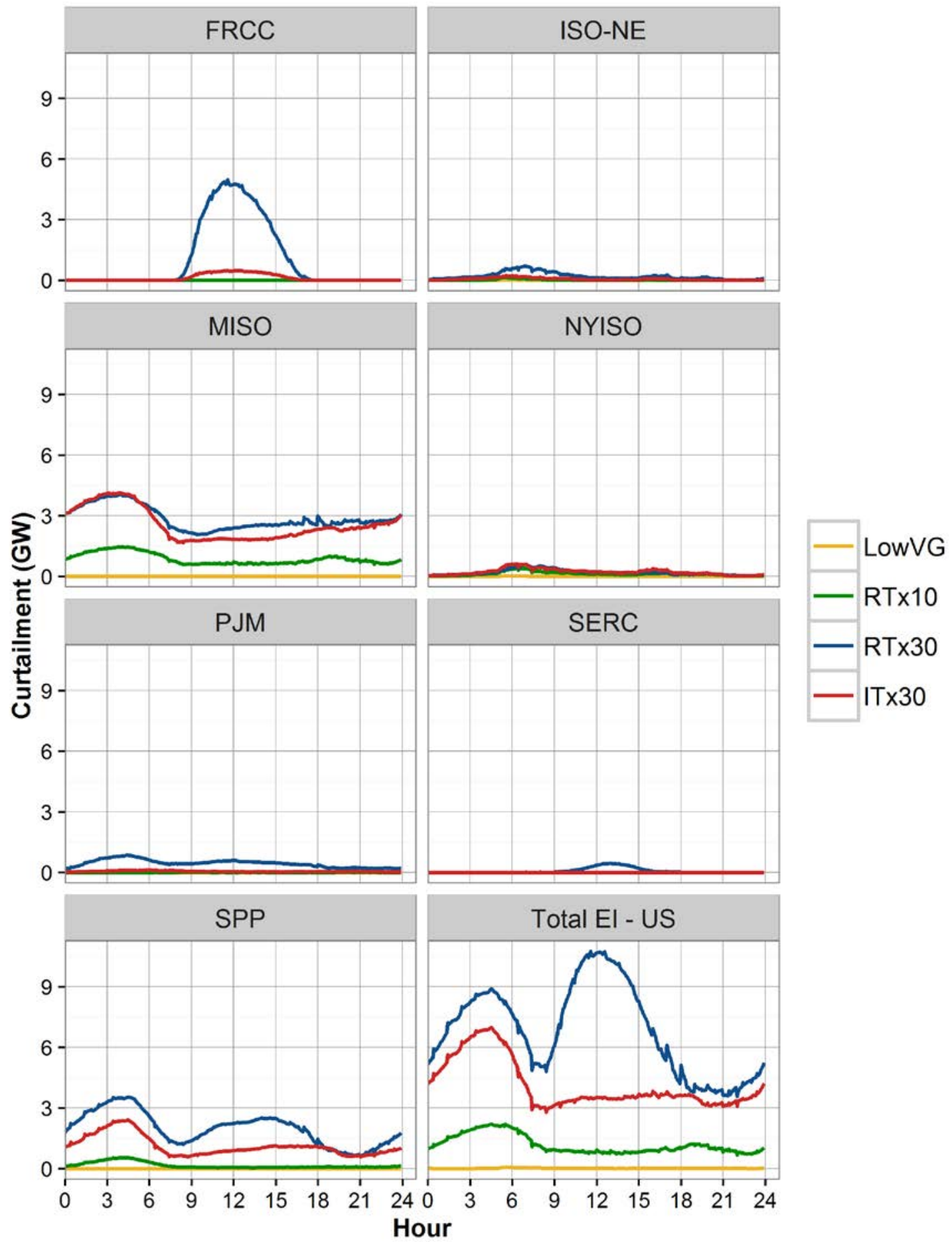

Figure 90. Annual average daily profiles of VG curtailment for the whole U.S. El and for each U.S. region

Figure 94 shows the seasonal average daily VG curtailment profiles for the whole U.S. EI and each of the U.S. regions. Most regions have low levels of VG curtailment. The VG curtailment in MISO for the RTx30 and ITx30 scenarios is very similar while SPP and FRCC both have much higher rates of VG curtailment in the RTx30. 


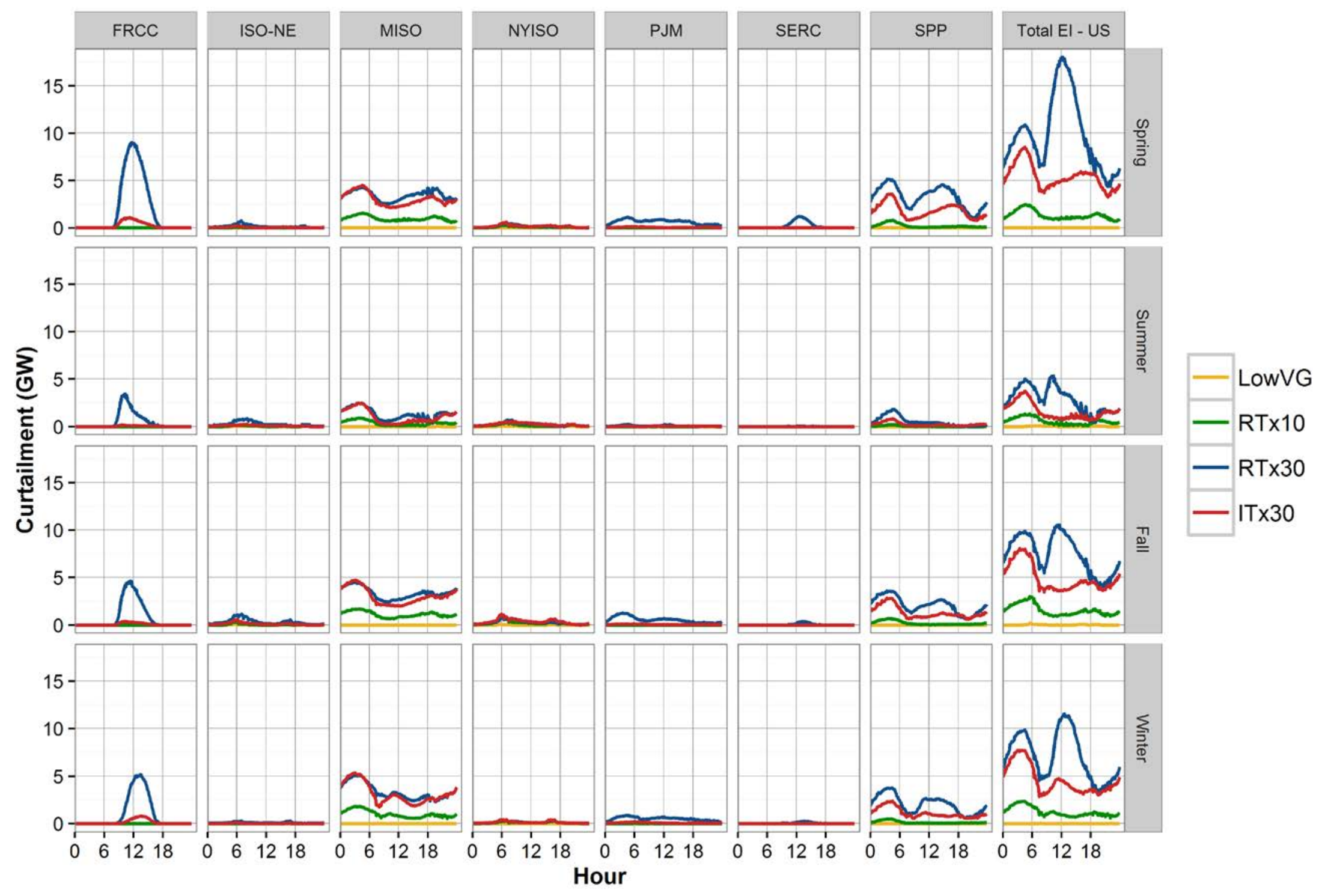

Figure 91. Seasonal average daily profiles of VG curtailment for the whole U.S. El and for each U.S. El region 


\subsection{Idle Thermal Capacity During Peak Demand}

All four scenarios used the same thermal fleet, which gives zero capacity value to the additional wind and PV resources in the RTx10, RTx30, and ITx30. To evaluate the extent to which using the same thermal fleet under-valued the capacity value of wind and PV, the amount of idle thermal (fossil- and nuclear-fueled) capacity during the peak demand for each of the scenarios was compared to the peak demand. This metric is similar to a traditional planning reserve margin, but only considers one year and disregards idle hydro capacity. Table 50 shows the U.S. EI coincident peak demand and the idle thermal capacity during the same time period, and the ratio of these two numbers. The LowVG has an idle ratio of 7\%, which is about half of the traditional $15 \%$ planning reserve margin target in many regions. Each of the higher-penetration scenarios have higher idle ratios, with the RTx30 having the highest at $30 \%$. These results suggest that the wind and PV capacity might displace a significant amount of thermal capacity without impacting reliability. However, multiple years of load and VG production data would be necessary to make this conclusion due to the possibility of differing VG production during peak demand.

Table 50. Idle Thermal Capacity During Peak Demand in Each Scenario

\begin{tabular}{|l|r|r|r|r|}
\hline Scenario & LowVG & RTx10 & RTx30 & ITx30 \\
\hline U.S. Peak Demand (GW) & 633 & 633 & 633 & 633 \\
\hline $\begin{array}{l}\text { U.S. Idle Thermal Capacity } \\
\text { During Peak Demand (GW) }\end{array}$ & 44 & 83 & 191 & 182 \\
\hline U.S. Idle Capacity Ratio & $7 \%$ & $13 \%$ & $30 \%$ & $29 \%$ \\
\hline
\end{tabular}

\subsection{Operating Costs and Emissions}

Operating costs - also called production costs or variable operations costs - are the costs associated with producing electricity and do not include capital costs. In ERGIS, operating costs include fuel, VO\&M, and startup costs. Wind and PV generation displace conventional thermal generation because of the low variable cost of VG. ${ }^{39}$ This displacement affects system operating costs and emissions.

Figure 95 shows the operating costs of the U.S. EI for each scenario, and Table 51 contains the numerical values. Fuel costs account for about $90 \%$ of the operating costs in each scenario. Increases in VG lead to lower operating costs in all scenarios, with the biggest component of savings coming from decreased fuel use. Total operating costs decreased by approximately $\$ 10$ billion (about 11\%) in the RTx10 and \$30-\$31 billion (about 31\%-32\%) in the ITx30 and RTx30 compared to the LowVG. Between the two $30 \%$ VG scenarios, the RTx30 had higher total operating costs by about $\$ 1$ billion. Fuel costs were lower in the RTx10, RTx30, and ITx30 by about $\$ 10$ billion, $\$ 29$ billion, and $\$ 30$ billion (11\%, 33\%, and 34\%), respectively. VO\&M costs followed the same trend and decreased by $7 \%, 22 \%$, and $22 \%$. In contrast, start costs were lower in the RTx 10 by $\$ 40$ million (3\%) but higher in the RTx30 and ITx30 by $\$ 400$ million $(30 \%)$ and $\$ 122$ million $(9 \%)$, respectively.

\footnotetext{
${ }^{39}$ Wind and PV were assumed to have no VO\&M costs, and no production tax credit (PTC) was assumed.
} 

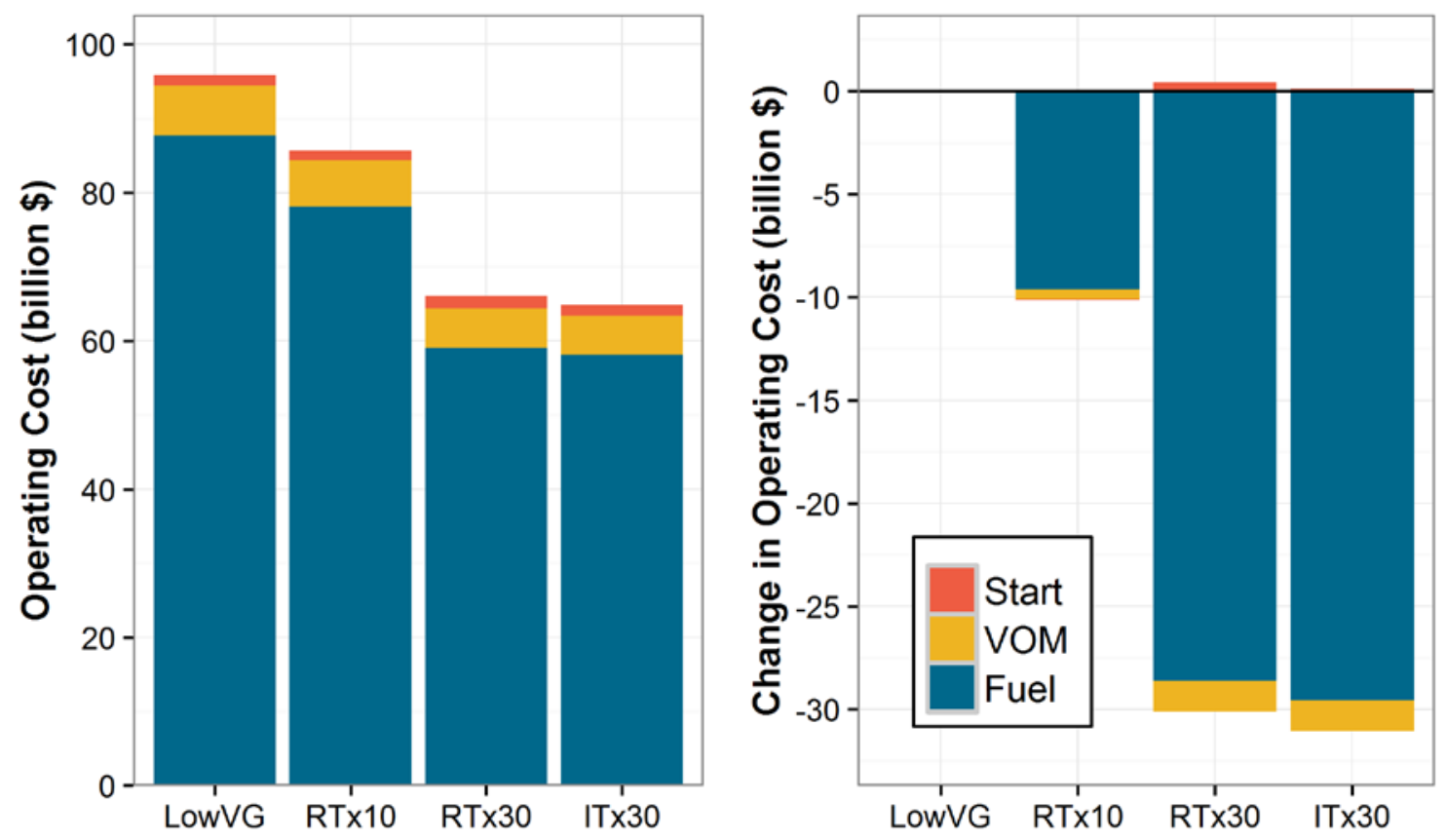

Figure 92. Annual operating costs for each of the scenarios

Note: Production costs do not include any fixed capital or PPA costs.

Figure 96 shows the carbon dioxide emission for each of the scenarios, and Table 51 contains the numerical values. No cost was assigned to carbon dioxide emissions. In the LowVG, almost two billion short tons of carbon dioxide were emitted by the U.S. EI. This was reduced to 1.3 billion tons in the $30 \%$ VG cases. Compared to the LowVG, the RTx10, RTx30, and ITx30 decreased emissions by about $11 \%, 31 \%$, and $33 \%$, respectively. 

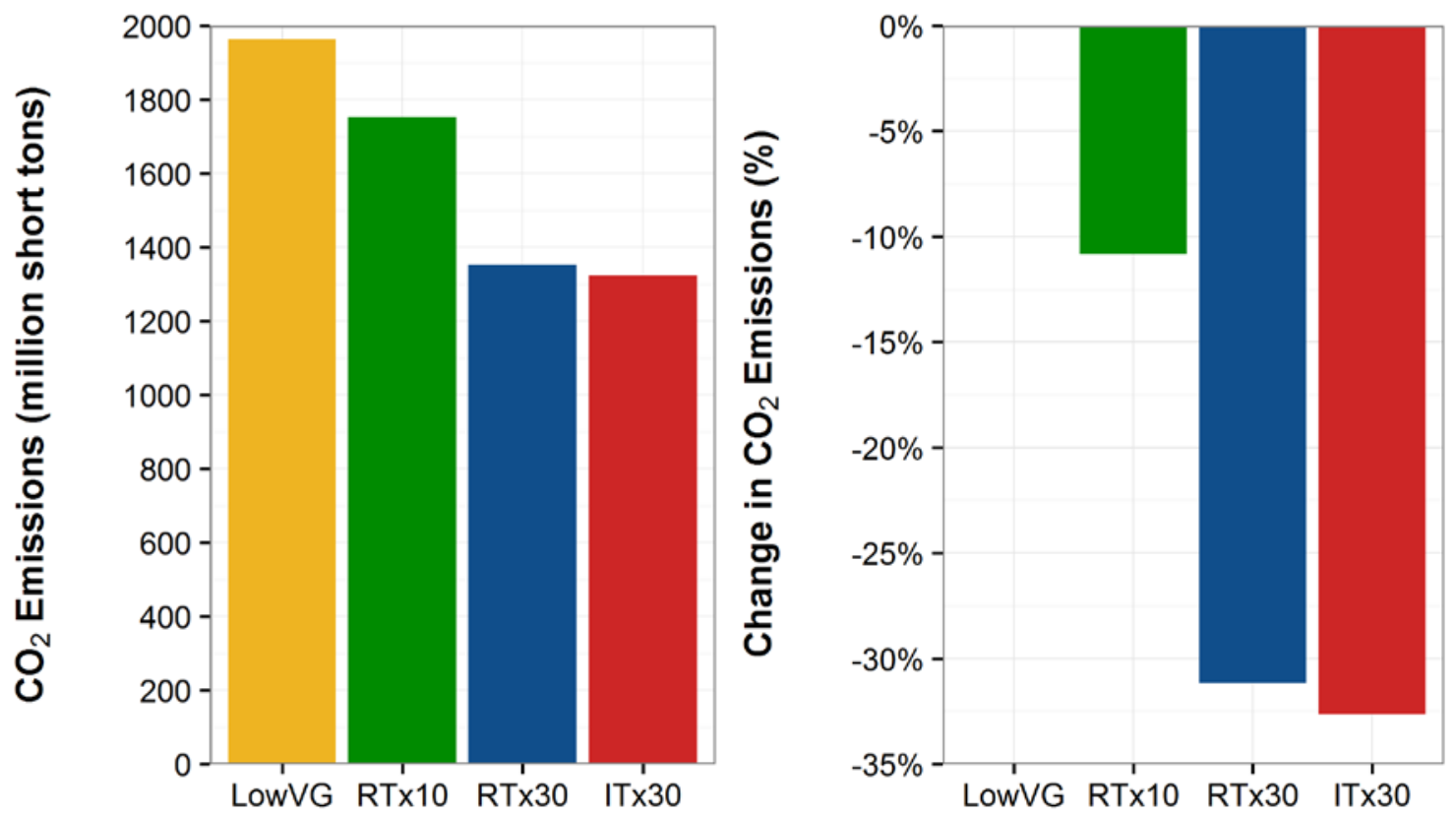

Figure 93. Carbon dioxide emissions for the U.S. El in each scenario (left), and change in carbon dioxide emission from the LowVG

Table 51 also shows the operating cost savings normalized by the increased amount of wind and PV generation in the RTx10, RTx30, and ITx30 scenarios over the LowVG scenario. The operating cost savings per additional potential wind and PV generation ${ }^{40}$ was $\$ 34.8 / \mathrm{MWh}$, \$34.2/MWh, and \$35.6/MWh in the RTx10, RTx30, and ITx30, respectively. With higher VG penetration these numbers typically decline, whereas in this case they actually increase. The increase in the ITx30 and the modest decrease in the RTx30, relative to the RTx10, is due to the differences in the types of VG (wind vs PV) and the different transmission expansions. The RTx10 is primarily wind, while the RTx30 incorporates much more PV. PV tends to offset more higher-cost gas generation than wind, which explains why the RTx30 per-unit savings does not decrease more than $\$ 0.8 / \mathrm{MWh}$ compared to the RTx10. The ITx30 contains a much larger transmission expansion, which allows more efficient use of all generator types (not just wind and PV). This explains why the ITx30 total and per-unit savings are slightly higher than the RTx30.

Finally, Table 51 shows the emissions reductions normalized by the amount of wind and PV generation in the RTx10, RTx30, and ITx30 scenarios. The ITx30 achieves slightly higher carbon dioxide emissions reductions than the RTx30 because it has higher VG penetrations in MISO and SPP, which include large amounts of coal generation.

\footnotetext{
${ }^{40}$ Potential generation is the sum of actual VG generation and VG curtailment.
} 
Table 51. Comparison of Operating Costs and Emissions with Increasing Wind and PV Generation

\begin{tabular}{|l|r|r|r|r|}
\hline Scenario & LowVG & RTx10 & RTx30 & ITx30 \\
\hline VG Generation (TWh) & 86 & 366 & 895 & 919 \\
\hline VG Penetration (\%) & 2.6 & 11.3 & 27.7 & 28.4 \\
\hline Fuel Costs (billion \$) & 6.8 & 6.3 & 5.3 & 5.3 \\
\hline VO\&M Costs (billion \$) & 1.4 & 1.3 & 1.8 & 1.5 \\
\hline Start Costs (billion \$) & 95.8 & 85.7 & 66.1 & 64.9 \\
\hline $\begin{array}{l}\text { Total Operating Costs } \\
\text { (billion \$) }\end{array}$ & NA & 34.8 & 34.2 & 35.6 \\
\hline $\begin{array}{l}\text { Operating Cost Savings per } \\
\text { Additional VG Potential } \\
\text { Generation 41 (\$/MWh) }\end{array}$ & & & & 58.1 \\
\hline $\begin{array}{l}\text { Carbon Dioxide Emissions } \\
\text { (million short tons) }\end{array}$ & 1,964 & 1,752 & 1,352 & 1,323 \\
\hline $\begin{array}{l}\text { Carbon Dioxide Emissions } \\
\text { Reductions per Additional } \\
\text { VG Potential Generation } \\
\text { (tons/MWh) }\end{array}$ & NA & 0.73 & 0.70 & 0.74 \\
\hline
\end{tabular}

\subsection{Operational Impact and Modeling Conclusions}

A variety of operational impacts were observed in the simulation of the EI under high penetrations of wind and PV. The introduction of VG alters the operation of thermal and hydro generating resources across all VG scenarios. While differences between operational impacts were observed at a regional level, interconnection-wide impacts were largely similar between scenarios, particularly with respect to annual metrics. Only selected operational periods in May, August, and November were presented because of their high penetrations of VG, high loads, and large net-load ramps. However the ERGIS simulations yielded detailed solution data for every time period simulated. Additional regional, 5-minute, and transmission analysis could be conducted to understand how interconnection-wide VG scenarios could impact other operational periods.

ERGIS modeling results are heavily influenced by the assumptions crafted with input from the ERGIS TRC. Taken as a whole, these assumptions helped the research team simulate the EI using realistic system operator practices based on 2014 operational practices. While this study does not capture all of the regional practices and individual resource parameters of the actual system, these assumptions represent a reasonable approach to simulating operations which is consistent with actual practice in many regions. This approach is in contrast to previous work where interregional trade was less constrained and new reserve products were included in simulations. As with other research of this scale, ERGIS includes centralized unit commitment and economic dispatch for the entire interconnection, a considerable assumption that could be improved in future work. ERGIS enforces significant market friction and does not allow wind, PV, or hydro to provide reserves. Decreasing market friction between regions and allowing wind,

\footnotetext{
${ }^{41}$ Potential generation is the sum of actual VG generation and VG curtailment.
} 
$\mathrm{PV}$, and hydro resources to provide reserves would likely impact production costs, operational impacts, and system-wide emissions. In future work, these assumptions could be tested to determine how the operational impacts change as a function of the operational practices used by the system operators. 


\section{Findings and Future Work}

We simulated four power system futures for the EI to determine the operational impact of integrating hundreds of gigawatts of wind and PV. In order to commit and dispatched the system to meet five-minute balancing requirements under a variety of wind and PV conditions our study investigated three power system futures that deviate from the current composition of the EI. The results of the production cost modeling indicate that the scenarios with high wind and PV have significantly different operational needs than our scenario with low wind and solar.

The first high-penetration scenario is the RTx10, which builds out a system that is designed to align with generally accepted builds necessary to meet state RPS in 2013. In the RTx30 and ITx30 scenarios, we build out additional wind and transmission to enable $30 \%$ of all load to be met with wind and PV. Our analysis at the 5-minute level indicates that the system can be balanced under a variety of operating conditions, including instantaneous generation from wind and PV in excess of 50\% of load. Our analysis shows that wind and PV resources are likely to reduce production costs and reduce system wide $\mathrm{CO}_{2}$ emissions by $\sim 30 \%$ when compared to the LowVG scenario. We used advanced modeling techniques and high performance computing systems to generate results at unprecedented fidelity and in ways that allowed us to eliminate many simplifying assumptions. Still, we did bound the scope of our analysis. This study did not investigate all aspects of the reliability problem faced by system planners and operators. It did not include an analysis of the capital costs for generation and transmission, or contingency analysis. Similarly, there is no analysis of the impact our natural gas expansions would have on natural gas infrastructure such as pipelines and gas storage.

These results were realized through a detailed representation of the EI and a balanced approach to modeling system flexibility. The model included 60,000 transmission lines, over 5,600 generator units, and all of the U.S. and Canadian portions of the EI and Québec Interconnection. Detailed generator constraints such as integer unit commitment and part-load inefficiencies were enforced to replicate actual UC\&ED practices used in many parts of the system. Current operational practices such as interregional friction, reserves and reserve sharing regions, and operational sequencing, were included to reflect the state of the present system. Additionally, flexibility from hydro resources was constrained as hydro units were unable to respond to wind and PV forecast errors. ERGIS did not include advanced flexibility options such as: new reserve products, an intra-day unit commitment, demand response, storage, or advanced thermal generator technology. However, the modeled system is more flexible due to an expanded transmission network, coincident retirement of coal and expansion of gas generators, and centralized UC\&ED.

Our analysis included the consideration of a variety of metrics including: starts, ramps, production costs, and VG curtailment. We also conducted a detailed analysis of five-minute operations for three challenging periods of systems operations, one for high load conditions, one for high VG generation, and one with a high net load ramp and forecast error. All load was served in all simulations and soft constraint violations such as reserves and interface limits were minimal. This analysis shows the importance of conducting annual simulations to understand seasonal operating conditions. It also highlights the importance of subhourly, five-minute analysis and need for additional research at this and shorter time domains. 
The results of this analysis led us to five conclusions with respect to the integration of wind and $\mathrm{PV}$ in the EI:

\subsection{The operation of thermal and hydro generation changes as wind and PV increase.}

The simulated wind and PV resources have seasonal and diurnal energy production patterns that impact how thermal and hydro resources behave in our model (Figure 94). Our simulations indicate that thermal plants will run fewer hours on an annual basis and cycle more frequently on a daily basis. Hydro and pumped storage resources shifted from a single peak per day to a morning and evening peak.
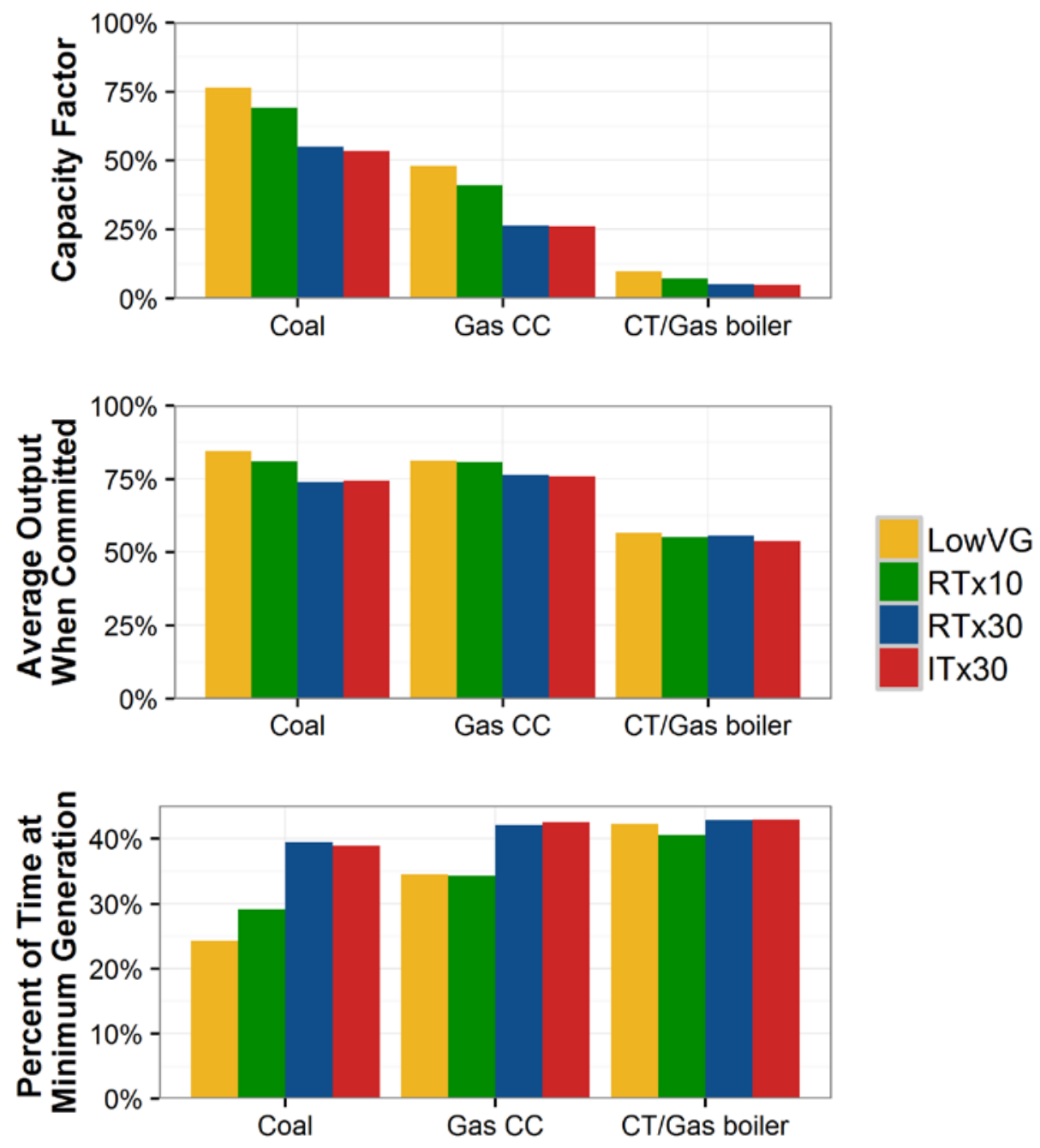

Figure 94. Capacity factors of thermal units for the total fleet in the U.S. El (top), Average output of committed units (middle) and percent of online time at minimum generation (bottom)

Capacity factors are calculated as the nameplate capacity of the generators by fuel type for all periods of the year divided by the total energy produced. 'Average output when committed' counts only committed units and calculates the output as a percentage of nameplate capacity of the unit. 
Wind and PV primarily displaced coal and combined cycle (CC) generation. ERGIS simulations show that annual wind and PV penetrations of $30 \%$ decrease coal, $\mathrm{CC}$, and combustion turbine (CT) capacity factors by $30 \%$ to $50 \%$.

Typical daily operational patterns also change in the $30 \%$ scenarios. Coal units increase time at minimum generation levels by $50 \%$ and $\mathrm{CC}$ units by $15 \%$. As wind and PV are added to the system, generation from thermal and hydro resources is shifted to different times of the day. Increased thermal and hydro utilization is observed in the hours before and after peak PV generation, see Figure ES-5. These changes in resource behavior are departures from the low renewable case, but can be accommodated with the modeled technologies and assumptions. Our findings on the operational patterns and ability to accommodate them are consistent with the broad consensus of renewable integration literature. Further analysis could help determine how these operational changes would impact the long-term financial viability of generators and fuel supply.

The variability of wind and PV cause other generators to ramp and start more frequently. In the $30 \%$ penetration scenarios, ramps per unit of energy by coal units increases by about a third and ramping by $\mathrm{CC}$ units increases by about a quarter. Starts for coal units increased by about $20 \%$, and starts for CC units increase by more than $40 \%$. CT starts decreased due to lower overall CT operation. While start costs reflected the additional wear and tear costs and emissions associated with cycling, they did not include other potential long-term impacts, such as increased forced outage rates due to increased wear and tear.

The regional impacts of high penetrations of $\mathrm{VG}$ varied due to regional differences in the thermal and hydro generation fleets and VG fleets. In general, the operational impacts tend to be greater when there is more installed PV in conjunction with less inter-regional transmission. These factors, coupled with hurdle rates ${ }^{42}$ between regions, cause some regions to have a greater reliance on balancing their own systems with local generation rather than using imports and exports.

\subsection{System operations at sunrise and sunset could follow different patterns.}

Our analysis shows that operations during the hours surrounding sunrise and sunset change more rapidly in the scenarios with higher renewables. The morning and evening load ramps have always been a challenging time for system operators and require expensive fast-starting, flexible resources before the addition of wind and PV to the system. After low load hours over night, morning load grows quickly in most parts of the country and typically stays high until peaking sometime in the afternoon or evening, depending on the season. Our simulations (Figure 95) indicate that the dispatch and commitment patterns of thermal and hydro resources will change as wind and PV are introduced to the system, creating a potential need for new operating rules, regulations, and practices to properly incentivize efficient operations. Around high wind and PV time periods, thermal and hydro plants tend to ramp faster and operate for shorter periods of time.

\footnotetext{
${ }^{42}$ Hurdle rates are an economic constraint in the model designed to approximate some of the economic inefficiencies, such as information asymmetry, that impact inter-regional electricity trade.
} 

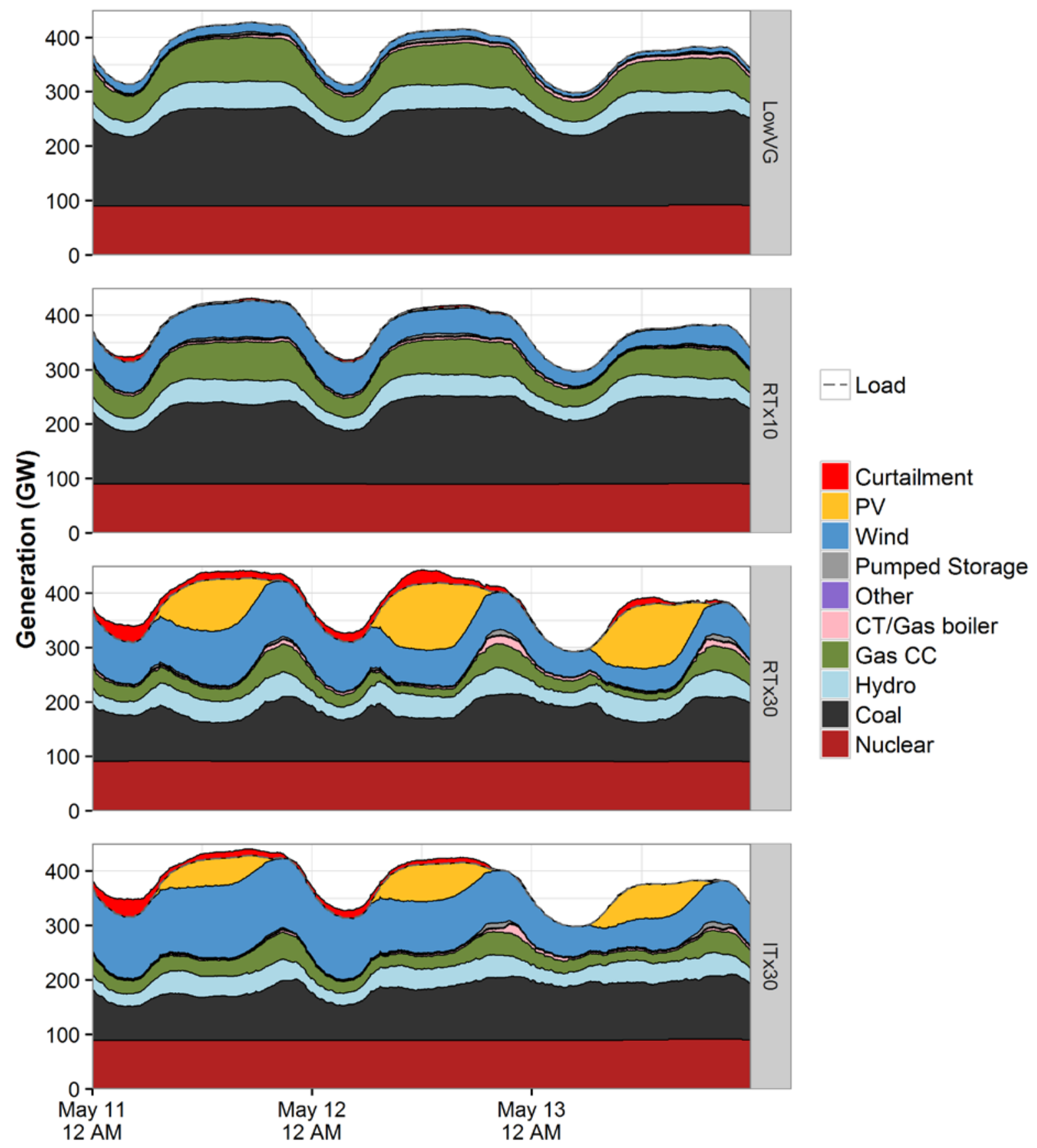

Figure 95. Dispatch for the U.S. El during high wind and PV conditions

Daily schedules for thermal and hydro units are quite different in the $30 \%$ cases than in the LowVG case. Instead of being committed and dispatched to meet a typical single peak load, our results indicate that thermal and hydro plants will be committed and dispatched to meet two netload peaks. The first occurs just before sunrise. The second occurs at sunset as PV generation decreases. 
Because our study was framed around annual average generation targets of $30 \%$ energy from wind and $\mathrm{PV}$, the modeled system experienced numerous periods of generation that were above and below the annual average. Peak penetration of VG approached $60 \%$ in some intervals while the minimum penetration from VG was approximately $10 \%$. During some periods of high wind and PV generation, two types of very low net load events may exist on the system. The first is a minimum net load at night, driven by wind generation. The second is a minimum net load during the day, driven by PV generation. The minimum net load levels are lower than normally observed by system operators and the addition of a minimum net load in the middle of the day is a significant difference from current system operating conditions.

\subsection{Transmission flows will likely change more rapidly and more frequently with higher penetrations of wind and PV.}

We observed that the average daily power flows between regions change substantially as more VG was added to the system. Some of the increased flows are due to the increased transmission buildout in the EI in our four scenarios, but changes due to the high penetration of VG are also evident. For example, in Figure 96 and Figure 97 we note the impact of PV on system-wide transmission flows. The PV in FRCC and SERC has a large impact on regional flows during the daylight hours that is not present in the LowVG. ${ }^{43}$ Not only do exports from the Southeast to the rest of the EI increase, but in the RTx30 (the scenario with the most PV capacity) exports from MISO and Hydro Québec to the high load regions on the U.S. East Coast decrease due to the cheaper power coming from SERC and FRCC.

Energy transfers between zones were limited based on hurdle rates and interface thermal limits. While the limits allowed us to simulate some of the economic and engineering constraints of inter-regional transmission utilization, this approach is not comprehensive. Our analysis relies on a single centralized UC\&ED, while actual UC\&ED is distributed across many entities in the EI. Furthermore, our model assumes a structure akin to an organized market. In reality, the EI features both organized and vertically integrated markets. This economic friction between markets is represented by hurdle rates and physical transmission limits are based on thermal constraints. However, no limitations on the ramp rates of flows between regions were modeled. In current operations, most regions have ramp rate limits for interchange schedules (PJM 2016). The exclusion of ramp rate increases the overall flexibility of the system.

\footnotetext{
${ }^{43}$ A video of these results is available on our website: www.nrel.gov/ERGIS
} 

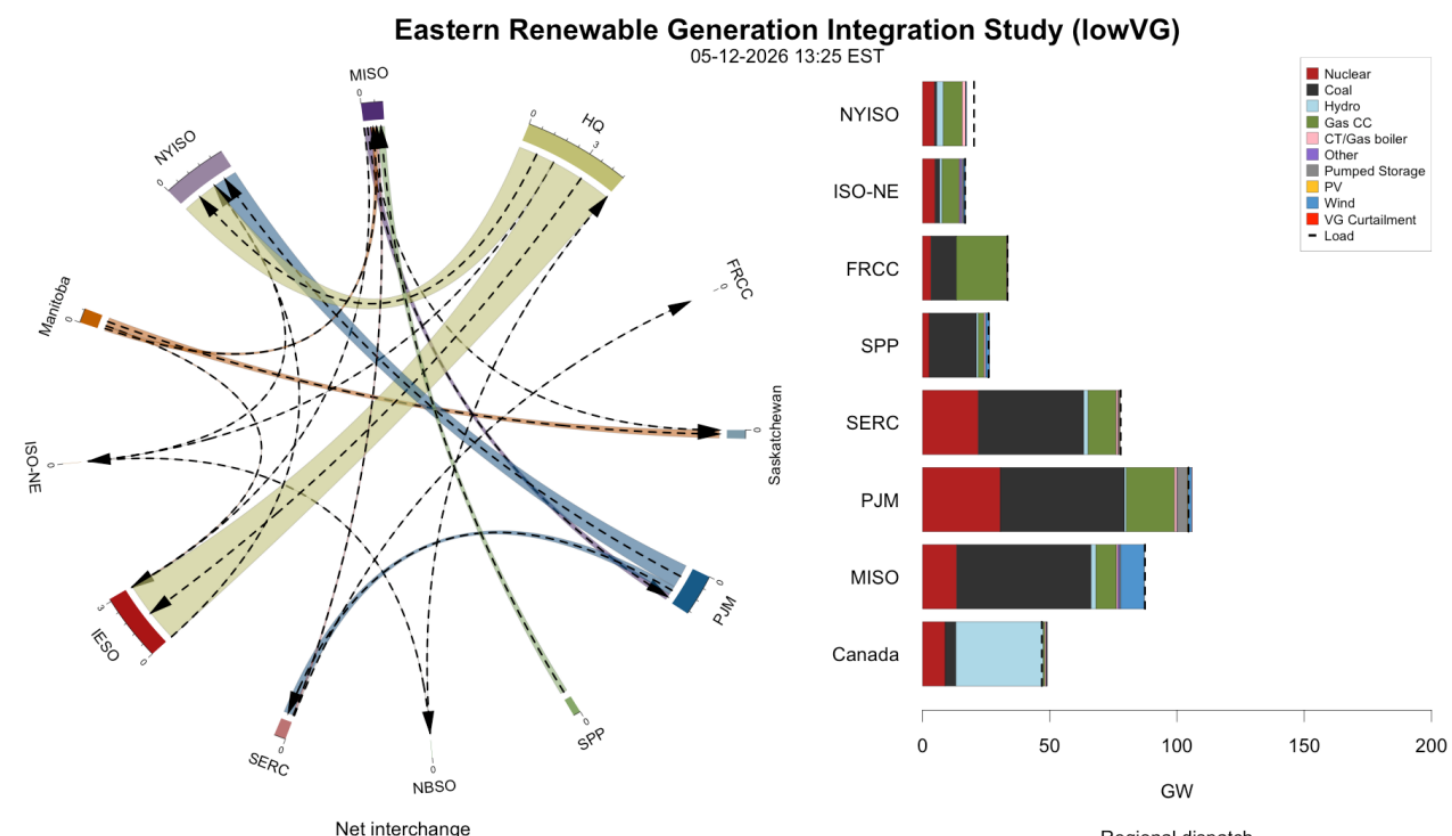

Regional dispatch

Figure 96. Net interchange in the LowVG on May 12, 2026 at 13:25

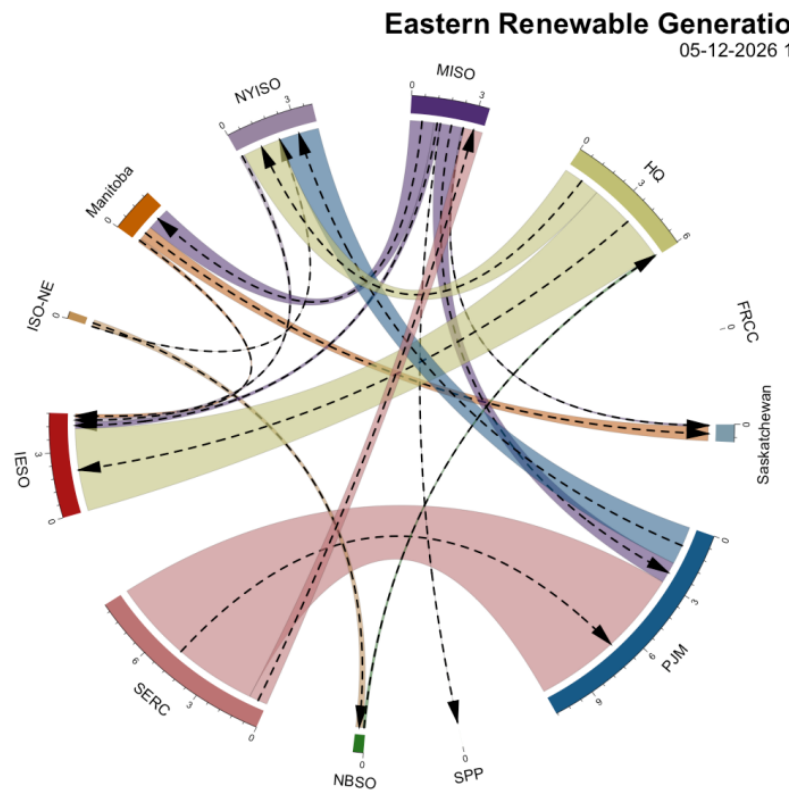

Net interchange

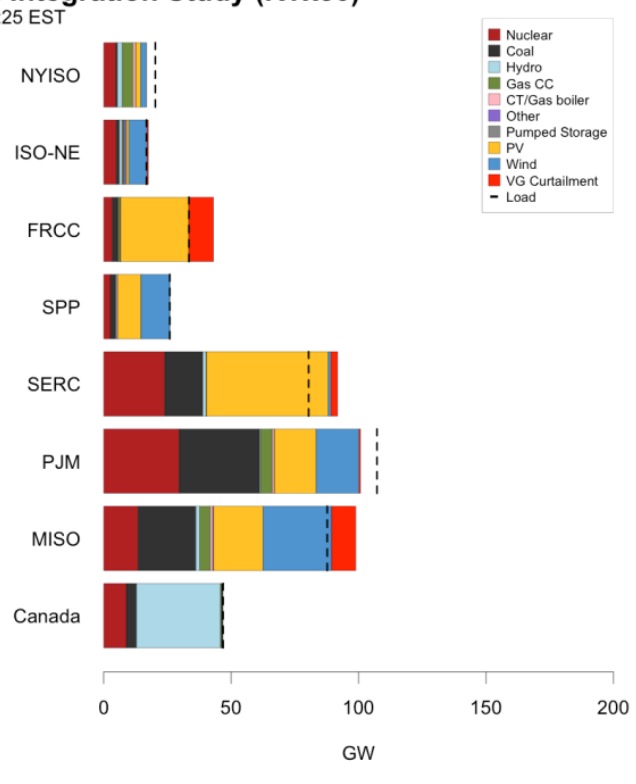

Regional dispatch

Figure 97. Net interchange in the RTx30 on May 12, 2026 at 13:25 
Daily patterns of power flow change from increased VG, and the rate at which the power flow changes is also impacted. However, both of the $30 \% \mathrm{VG}$ scenarios show a more rapid change in the power being transferred between regions than the lower penetration scenarios. This suggests that greater coordination between regions to handle faster changes on interface flows may have an economic benefit if large amounts of wind and PV are installed throughout the interconnection.

Additional analysis could more accurately represent economic friction between markets. Similarly, additional work on computational approaches and methods could enable an even more complete list of transmission constraints to be included in large network models such as the EI. Finally, ramp rate limits on transmission lines should also be considered in future work to determine if there are limitations on the ability of systems to use trading with neighboring regions to accommodate net load ramps. If the actual utilization of interregional transmission is more limited than allowed in this study, additional analysis could explore other possible approaches for accommodating high penetrations of VG. Solutions may include utilizing more flexibility in the thermal fleet (ramping or starting/shutting down local generators) or curtailing more of the VG.

\subsection{The operating practices of generators and transmission operators will be critical to realizing the total technical potential of the interconnection.}

The ability of the EI to integrate and balance hundreds of GW of wind and PV generation depends on generator and transmission operators offering their capabilities to the system operator. In ERGIS, we used detailed modeling assumptions and advanced methods that were vetted by a TRC to determine whether our model could commit and dispatch resources to balance load at a 5-minute intervals for an entire year of simulated operations. We show that the system can be committed and dispatched to balance the system in a variety of conditions, including high load, high VG, and during extreme ramping conditions. However, we did not investigate whether transmission and generation operators will have sufficient incentives to provide the necessary ramping, energy, and capacity services for futures like the ones we studied. While ERGIS shows it is technically possible to balance periods of instantaneous VG penetrations that exceed 50\% for the EI (Figure 98), the ability of the real system to realize these futures may depend more on regulatory policy, market design, and operating procedures. 


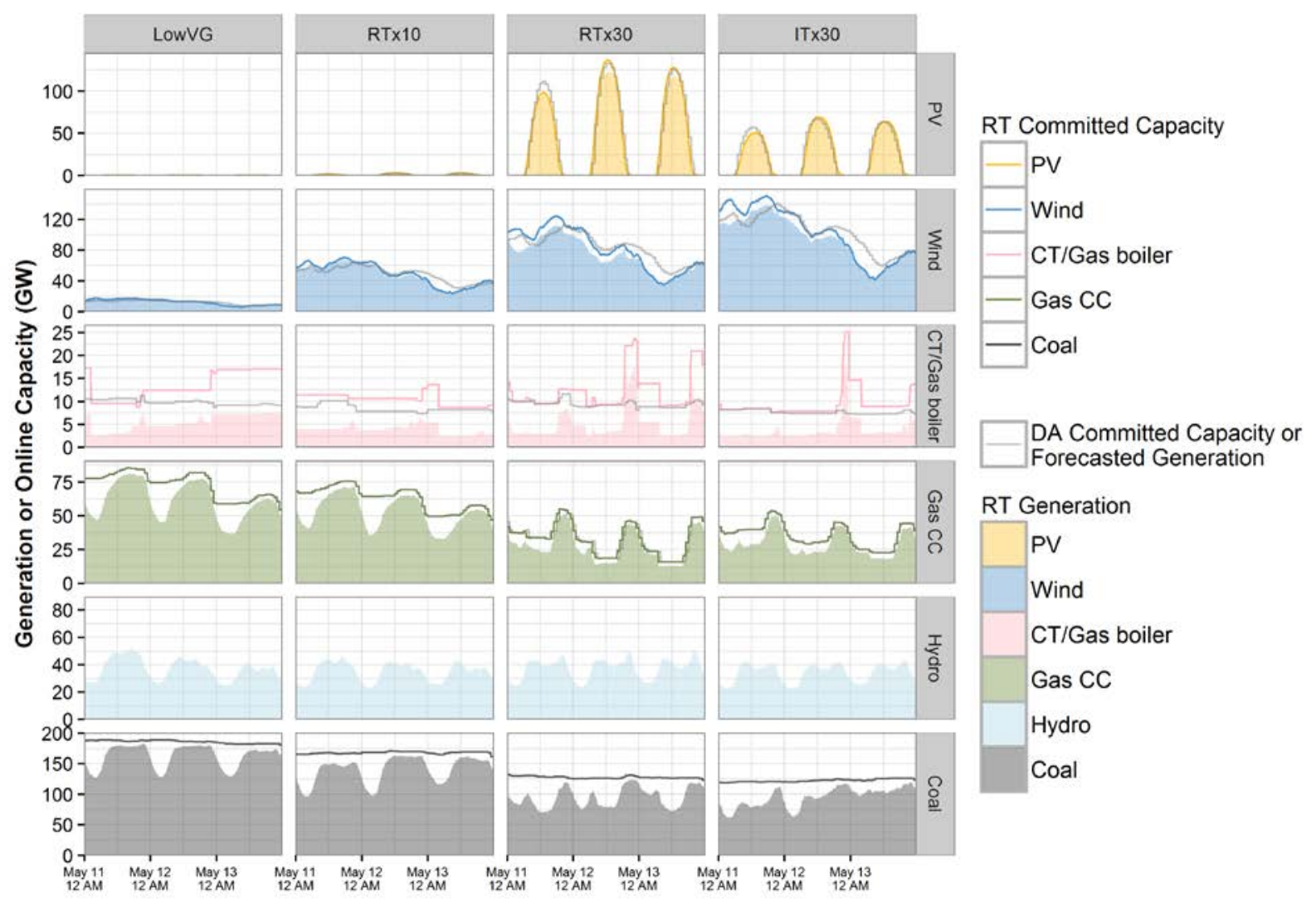

Figure 98. Day-ahead and real-time results for a period of high wind and PV generation

We assume hydro, pumped storage, and thermal resources are willing to offer their capabilities at any time. In reality, many of the operating practices of generators are based on historical behavior, equipment limitations, and regulatory structures focused on peak load conditions. In ERGIS we observed a shift in thermal, hydro, and pumped storage plant operations from a single peak to two shorter and steeper peaks during high VG conditions. This change has implications for system operating practices. In futures with high amounts of wind and PV, system and plant operators will need to focus their attention on different times of day and could expect to cycle or ramp their resources more frequently. If adequate short term opportunities and regulatory structures are not in place to incentivize this flexibility, resources may exit the market or prefer not to make their full flexibility available to the system operator, compromising the ability of the system operator to manage the types of conditions simulated in this study. These new flexibility requirements may also provide opportunities for technologies like demand response or energy storage to support the management of the conditions explored in this study.

Our analysis also indicates that futures with high amounts of VG will likely benefit from multiregional coordination. Our model assumes that there is a single mathematical formulation used to commit and dispatch the entire power system. As such, we observed instances where high levels of wind and PV appear to be transferred across more than one region. This is because the model saw economic value and the technical ability to transfer energy very long distances. In reality the operations of the EI and the market are currently managed by a variety of entities. While operations between those entities are coordinated to some extent, this coordination will likely 
become more important in the future. While we have modeled some of the economic and technical constraints on interchange between regions, market participants will likely require significant, additional coordination across multiple areas in order to act on resource availability that is multiple regions away.

Finally, our model assumed a common thermal generating fleet across scenarios regardless of renewable penetration. This means that in our high wind and PV cases, the RTx30 and ITx30, there is likely more thermal generating capacity in our model than would be expected on the actual system. The ability of resources to obtain sufficient revenues from the new operating patterns will likely impact resource adequacy. Given the reduced utilization of many generators, it is unclear whether energy market revenue alone will be sufficient to keep units from retiring. Additional analysis of these long-term economic impacts is essential to determine the true feasibility of the simulated scenarios.

\subsection{Advanced visualization tools are helpful for understanding spatially and temporally rich models.}

New visualization tools help to ensure model accuracy and provide analytical insights. Power system futures with high levels of wind and PV require simulations with large datasets featuring high spatial and temporal resolution. The large amounts of data create the need to programmatically create, manage, and analyze big data sets. We created two visualization tools in the statistical software package, $R$, to manage this data, ensure accuracy, and provide timely analysis (Figure 99). ${ }^{44}$
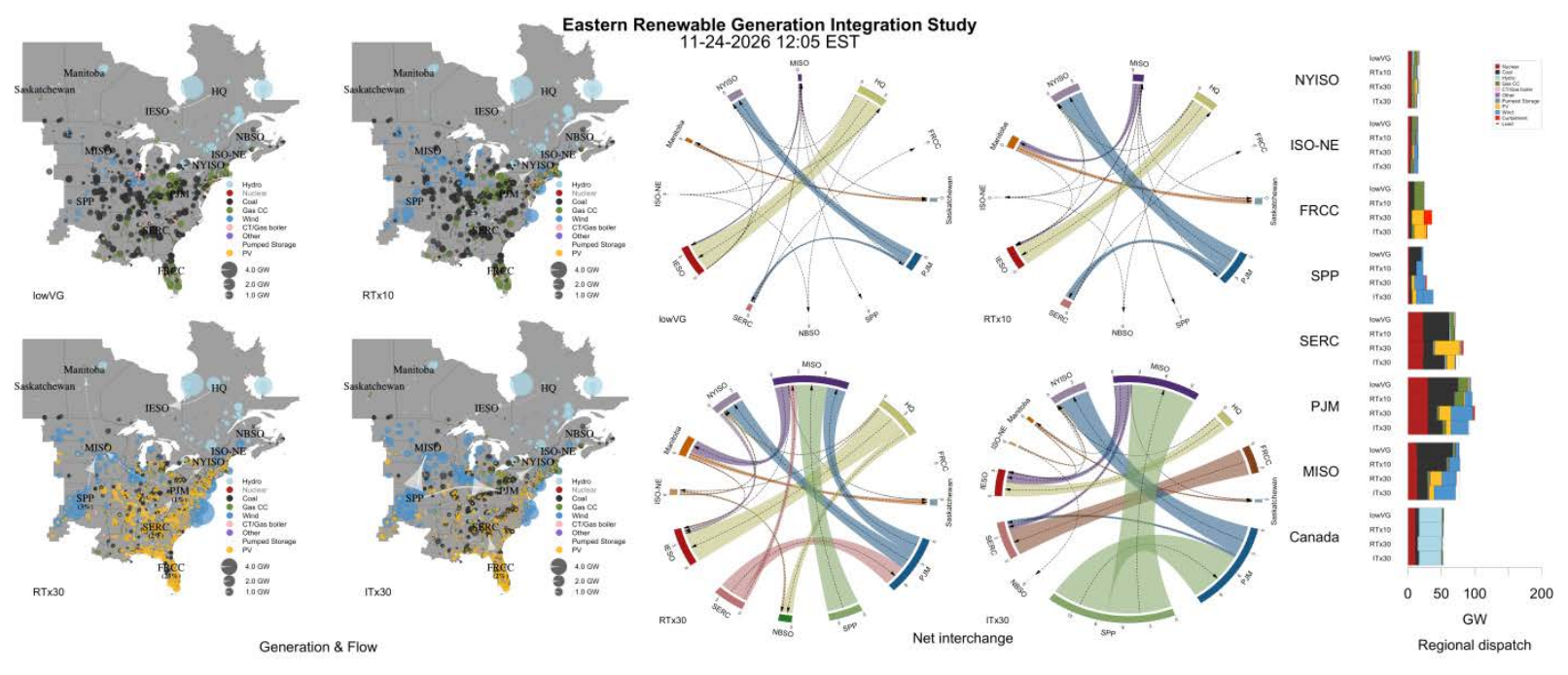

Figure 99. Sample screen from "kaleidoscope" visualization tool, one of two tools developed for ERGIS

These tools assisted us in two ways. First, they streamlined the analysis process by enabling the rapid visualization of numerous interim simulations conducted for the study. This enabled the team to identify anomalies and verify results. Second, these tools helped us understand how the model was using coordinated operations across multiple regions to manage the changing

\footnotetext{
${ }^{44}$ A sample of the kaleidoscope visualization for large displays is available at our website: www.nrel.gov/ERGIS
} 
conditions presented by wind and PV. In animations created using kaleidoscope, we observed how varying wind and solar generation drives interchange across multiple regions.

The broader research and industry community may use these tools and contribute improvements of these tools via the open source platform GitHub. ${ }^{45}$ We hope that others will be able to use these research grade tools to manage the results of production cost model simulations, visualize results, explore data and results in new ways, and reduce the amount of time necessary to validate and act on analysis.

\subsection{Opportunities for Future Work}

This study demonstrates technical potential for the Eastern Interconnection to balance supply and demand at 5-minute intervals with up to $400 \mathrm{GW}$ of wind and solar generation added to the system. Future work could help determine the economic and market potential for high VG operation of the EI. Potential areas of future work include

- Study of regional commitment and dispatch via geographic decomposition

- Further reduction in model run time via geographic decomposition

- Regional seams coordination

- Revenue sufficiency and resource adequacy

- Co-optimized thermal, renewable and transmission expansion

- Computationally-efficient representation of greater numbers of transmission constraints, including both flow and ramp rate constraints.

- Dynamic stability, frequency response and inertia

- Impact and value of enhanced system flexibility, such as:

○ Demand response

- Electricity storage

- Operational practices

o Optimization and deployment of reserves.

${ }^{45}$ Links to the GitHub repositories are available on our website: www.nrel.gov/ERGIS 


\section{Appendix A: Solar Irradiance Statistical Analysis}

There are four measurements we conduct on a finished data set: coherence, ramp distributions, capacity factor, and area aggregation of time series. This section describes the statistical analysis. Additional results from the statistical analysis are included in Appendices A through $\mathrm{C}$.

1. Coherence. As mentioned above, coherence is the measure of correlation of clearness index ci ramp (change in ci over time $\mathrm{t}$ ) between sites, across all time lags. Typically we look at 1, 5, 10, 30, 60, and 180 minute lags. The 60 and 180 minute ci ramps are determined by the satellite data since the algorithm is forced to go through each satellite data point at the top of the hour. The other time lags measure the coherence in the algorithm itself. Figure 99A shows a coherence measurement made by Andrew Mills and Ryan Wiser (2010) on a network of solar sensors in Southern Great Plains; Figure 99B shows a coherence measurement from the WWSIS II dataset generated by the sub-hour irradiance algorithm in Hummon et al. (2012). Figure 100 shows the locations of several regions in the ERGIS solar dataset. Figure 101 shows the coherence measurement for the ERGIS dataset generated by the sub-hour irradiance algorithm. Table 52. Fit parameters for the different regions mapped in Figure 10. Table 52 gives the fit parameters for the regions shown in Figure 100. 

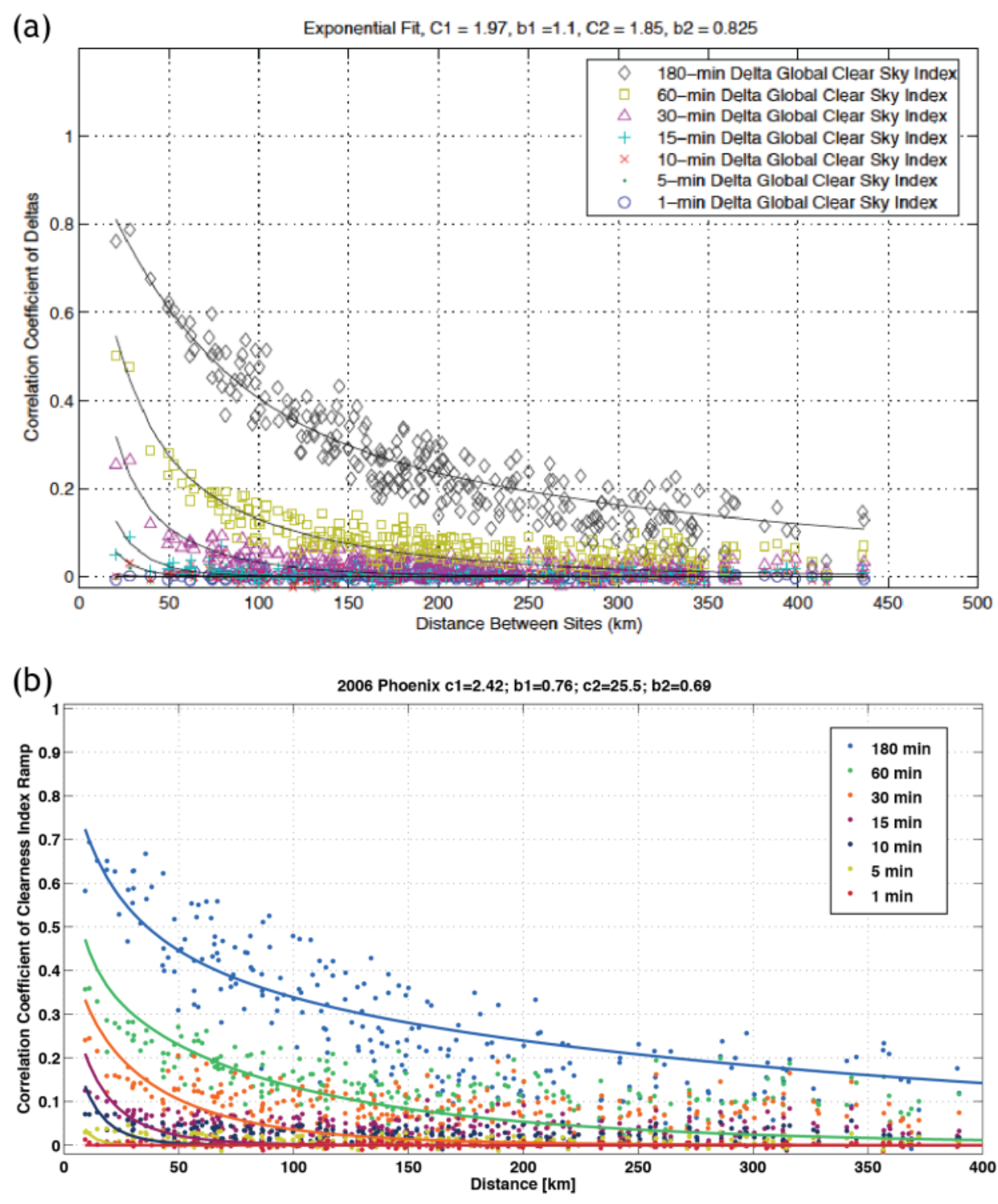

Figure 100. Coherence of dataset. (a) Mills et al. measured the coherence of the Southern Great Plain network (measured data). (b) WWSIS II modeled data near Phoenix, AZ. The coefficients of the exponential fit have been shown to be related to the mean size and speed of clouds in the region, thus different coefficients between sites are expected. 


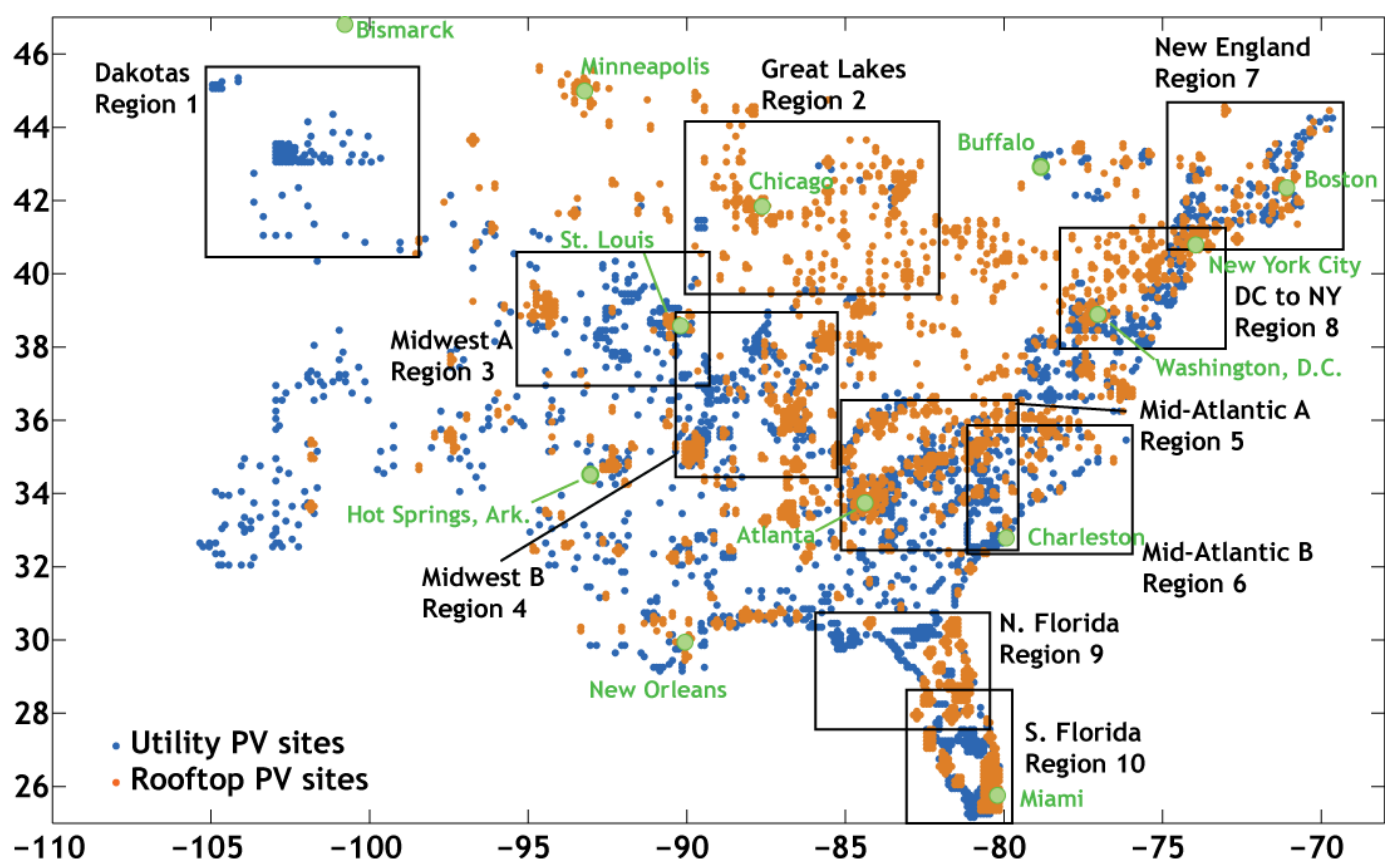

Figure 101. Utility and rooftop PV sites for ERGIS, grouped by region.

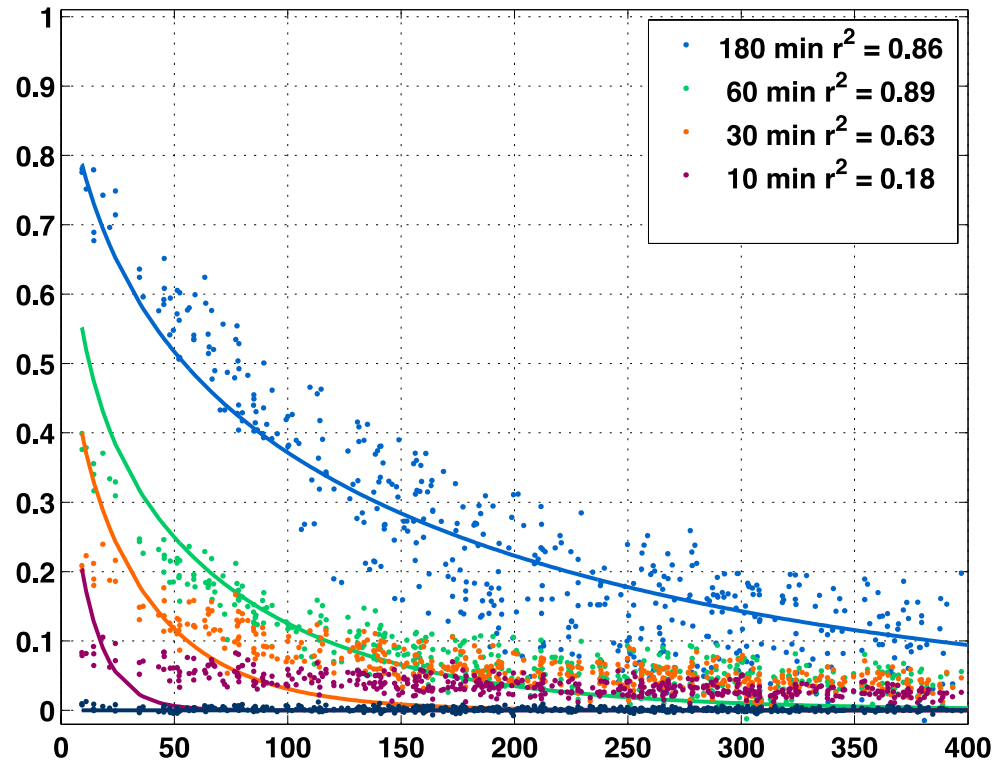

Figure 102. Coherence of ERGIS utility and distributed data for the Mid-Atlantic A region. The coefficients of the fits are shown in the figure legend. 
Table 52. Fit Parameters for the Regions Mapped in Figure 10.

\begin{tabular}{lcccc}
\hline Fit Parameters & C1 & C2 & B1 & B2 \\
\hline Dakotas & 1.703 & 14.363 & 0.825 & 0.696 \\
Great Lakes & 1.146 & 12.730 & 0.889 & 0.711 \\
Midwest A & 0.647 & 11.988 & 1.024 & 0.715 \\
Midwest B & 0.623 & 14.277 & 1.031 & 0.678 \\
Mid-Atlantic A & 1.115 & 20.400 & 0.937 & 0.641 \\
Mid-Atlantic B & 1.271 & 14.563 & 0.883 & 0.734 \\
New England & 1.698 & 6.664 & 0.752 & 0.879 \\
DC to NY & 1.121 & 19.352 & 0.923 & 0.633 \\
North Florida & 0.981 & 30.243 & 1.002 & 0.594 \\
South Florida & 1.681 & 26.752 & 0.911 & 0.653 \\
\hline
\end{tabular}

Correlation fit parameter are used with the equation $c_{1} \exp \left(-d^{-b 1 / t}\right)+c_{2} \exp \left(-d^{b 2 / t}\right)$ where $d$ is the distance between the sites in kilometers.

2. Ramp Distributions. A ramp is defined as the change in clearness index over a length of time. The algorithm randomly draws ramps from distributions derived from the groundbased measured sources. We check that individual sites of modeled data have a distribution of ramps that is appropriate for the types of clear sky behavior at that location. Sites that are heavily weighted by clear sky conditions have fewer large ramps, whereas sites with cloudy sky conditions have fewer small ramps. Figure 102 shows the histogram of Clearness Index (CI) for a single site at $(-80.95,26.95)$, a cluster of sites (S. Florida), and aggregation of Utility PV sites over the ERGIS region. Figure 103 shows the cluster regions used to find the distributions of ramps of utility PV data shown in Figure 102. Ramp distributions are best compared to measured data, but in the absence of measured data at specific location such as this case, an approach we might consider might be to "build" an annual distribution of ramps based on our database of satellite data and ground-measured data to compare to the simulated data.

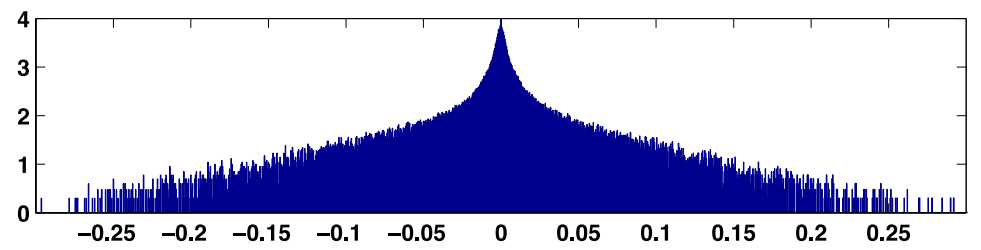

South Florida

cluster

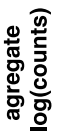

ci power ramps (unitless)

Figure 103. Histograms of Clearness Index (CI) for a single site at $(-80.95,26.95)$, a cluster of sites, and aggregation of the utility PV sites over the entire ERGIS region 


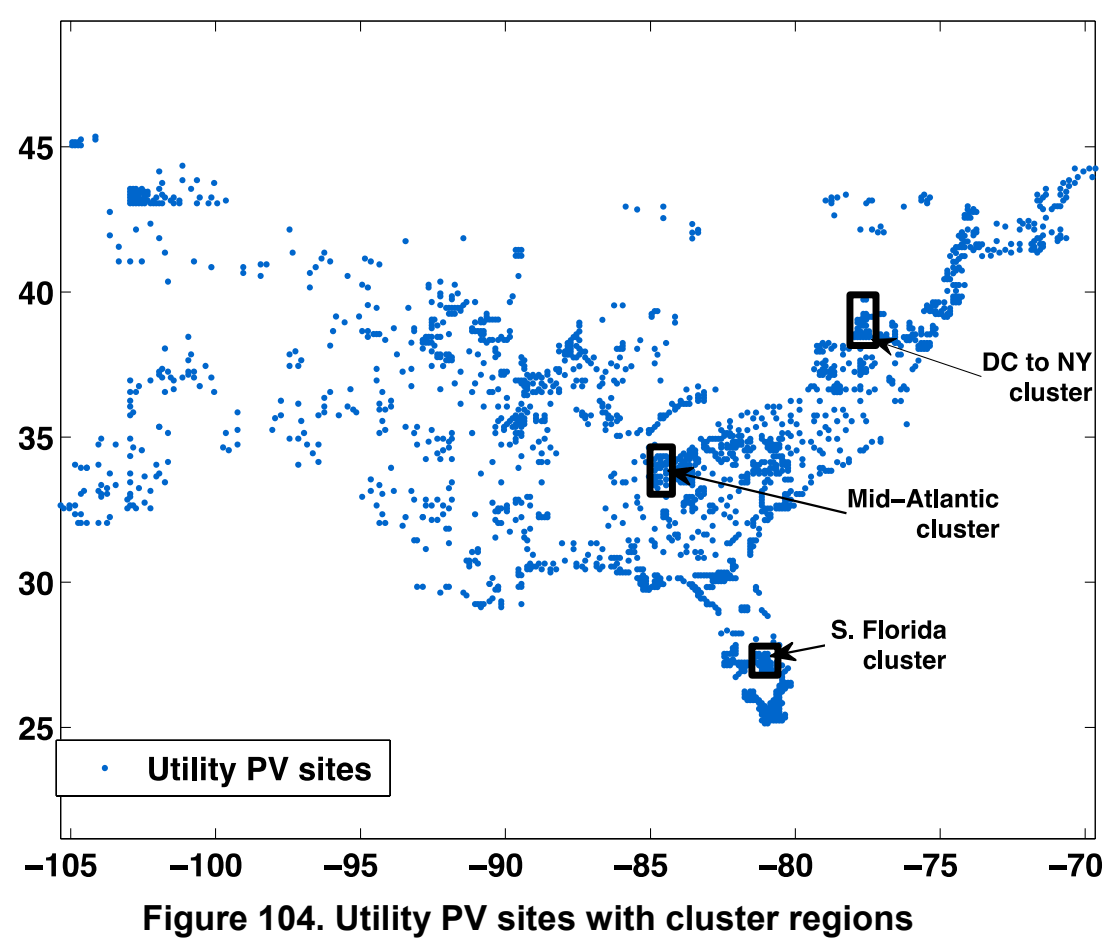

3. Capacity Factor. Capacity factor (CF) is the annual measure of energy produced divided by the theoretical energy produced (DC nameplate capacity times 8760 hours). We check that the integral of the 1-minute dataset is within $1 \%$ of the satellite data for that location, as well as checking the intermediate datasets (5-minute average, 30-minute average). Table 53 shows the capacity factor of selected DVP and UPV site clusters.

Table 53. Capacity Factor of Selected DPV and UPV Clusters

\begin{tabular}{lc}
\hline Cluster name & Capacity Factor \\
\hline DC to NY (utility) & 0.16 \\
Mid-Atlantic (utility) & 0.17 \\
South Florida (utility) & 0.19 \\
Midwest A (rooftop) & 0.08 \\
Midwest B (rooftop) & 0.12 \\
Midwest C (rooftop) & 0.11 \\
All ERGIS Utility PV & 0.17 \\
All ERGIS Rooftop PV & 0.11 \\
\hline
\end{tabular}

4. Time Series and Aggregation. Visual inspection of the simulated time series of solar power output is an important validation step. While not statistical in nature, the look of the time series is the first test the simulated data set must pass. In addition, when we aggregate (sum) solar power sites in a region, we expect that time series to look relatively smoother. The ramp distributions, as a function of aggregation, can confirm the reduction in variability. Figure 104 demonstrates an example of a 3-day time series of the power output for a single site at $(-80.95,26.95)$, a cluster of sites, and aggregation of utility PV data for sites over the ERGIS region. 

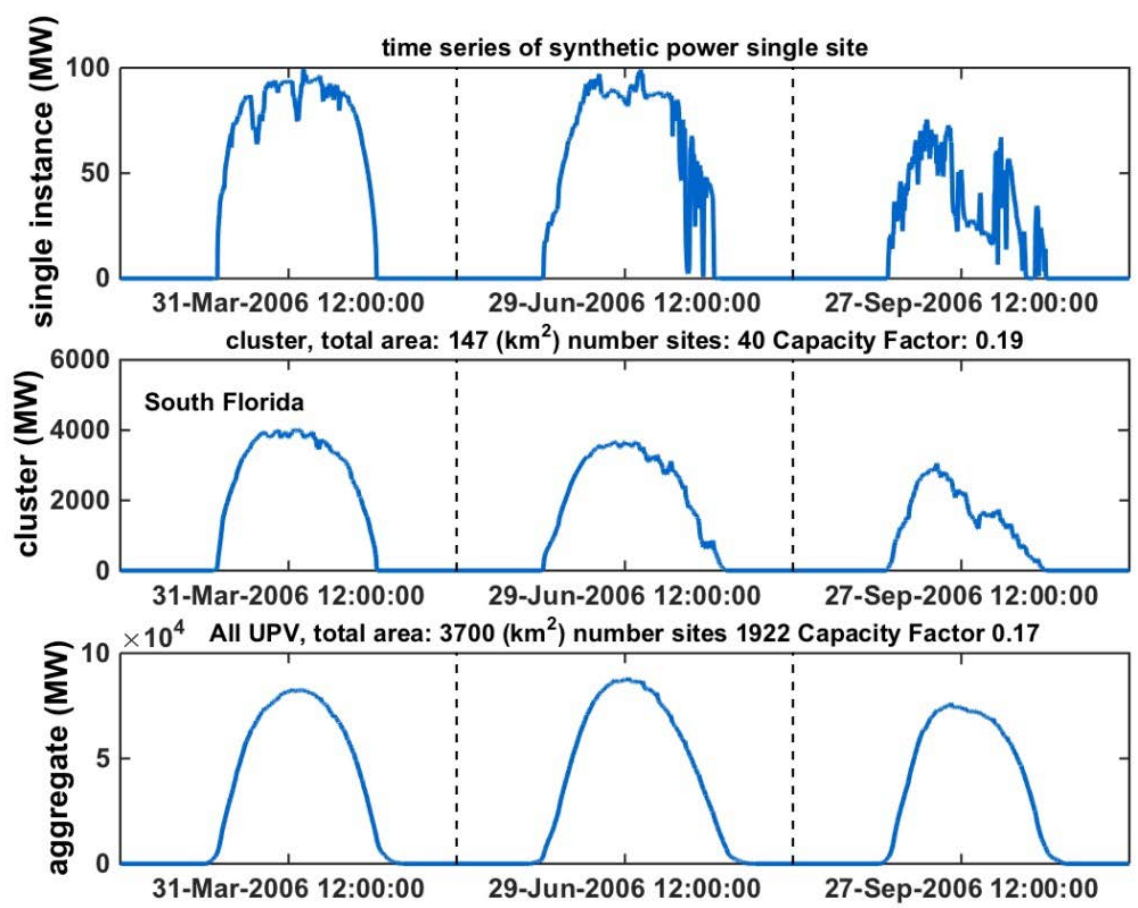

Figure 105. Example showing a 3-day time series of the power output for a single site at $(-80.95$, 26.95), a cluster of sites (South Florida - see Figure 103), and aggregation of utility scale sites over the ERGIS region.

The analysis of the coherence, ramp distributions, capacity factor, and area aggregation of time series indicated successful implementation of the sub-hourly irradiance algorithm for the ERGIS dataset. Additional results from the statistical analysis are included in Appendices A through C. 


\section{Appendix B: Regional Load Scale Factors}

Table 54. Final Load Scale Factors for Each of the ERGIS Sub-Regions.

\begin{tabular}{|l|r|}
\hline ERGIS Sub- & $\begin{array}{r}\text { 2006-2026 } \\
\text { Region } \\
\text { Increase }\end{array}$ \\
\hline FRCC & $11.08 \%$ \\
\hline HQ & $3.05 \%$ \\
\hline IESO & $3.05 \%$ \\
\hline ISO-NE & $3.05 \%$ \\
\hline Manitoba & $12.73 \%$ \\
\hline MISO1 & $12.73 \%$ \\
\hline MISO2 & $5.64 \%$ \\
\hline MISO3 & $5.64 \%$ \\
\hline MISO4 & $5.03 \%$ \\
\hline MISO5 & $18.10 \%$ \\
\hline MISO6 & $8.02 \%$ \\
\hline MISO7 & $2.55 \%$ \\
\hline MISO8 & $18.10 \%$ \\
\hline MISO9 & $18.10 \%$ \\
\hline NBSO & $3.05 \%$ \\
\hline MISO SD & $12.73 \%$ \\
\hline NYISO A-F & $4.10 \%$ \\
\hline NYISO G-I & $2.37 \%$ \\
\hline NYISO J-K & $0.86 \%$ \\
\hline PJM Chicago & $8.02 \%$ \\
\hline PJMEMAAC & $7.25 \%$ \\
\hline PJMMAAC & $7.25 \%$ \\
\hline PJMROR & $8.02 \%$ \\
\hline PJMSouthBend & $2.55 \%$ \\
\hline Saskatchewan & $12.73 \%$ \\
\hline SERC-Duke & $16.77 \%$ \\
\hline SERC-SOCOAL & $15.23 \%$ \\
\hline SERC-SOCOFL & $15.23 \%$ \\
\hline SERC-SOCOGA & $15.23 \%$ \\
\hline SERC-TVA & $13.77 \%$ \\
\hline SPP-N & $9.58 \%$ \\
\hline SPP-NE & $12.73 \%$ \\
\hline SPP-S & $20.80 \%$ \\
\hline
\end{tabular}




\section{Appendix C: Statewide Wind and PV Resources}

Table 55. Wind and PV Capacity by State and Scenario

\begin{tabular}{|c|c|c|c|c|c|c|c|c|}
\hline \multirow[b]{2}{*}{ State } & \multicolumn{2}{|c|}{ LowVG } & \multicolumn{2}{|l|}{ RTx10 } & \multicolumn{2}{|l|}{ RTx30 } & \multicolumn{2}{|l|}{ ITx30 } \\
\hline & Wind & PV & Wind & PV & Wind & PV & Wind & PV \\
\hline $\mathrm{AL}$ & 0 & 0 & 0 & 0 & 0 & 7,986 & 0 & 2,651 \\
\hline AR & 0 & 0 & 0 & 0 & 0 & 3,916 & 839 & 1,619 \\
\hline CT & 0 & 2 & 900 & 2 & 1,018 & 1,241 & 1,781 & 513 \\
\hline $\mathrm{DE}$ & 2 & 26 & 319 & 96 & 3,759 & 443 & 1,978 & 197 \\
\hline $\mathrm{FL}$ & 0 & 85 & 0 & 85 & 0 & 56,771 & 0 & 37,970 \\
\hline $\mathrm{GA}$ & 0 & 6 & 0 & 6 & 0 & 16,326 & 0 & 6,718 \\
\hline $\mathrm{IA}$ & 1,994 & 0 & 4,790 & 0 & 8,434 & 891 & 11,746 & 422 \\
\hline $\mathrm{IL}$ & 2,935 & 47 & 7,031 & 48 & 17,499 & 3,812 & 21,556 & 1,628 \\
\hline IN & 1,639 & 0 & 2,415 & 0 & 4,389 & 4,217 & 8,613 & 1,965 \\
\hline KS & 1,912 & 0 & 5,054 & 1 & 10,110 & 1,128 & 18,870 & 1,373 \\
\hline $\mathrm{KY}$ & 0 & 2 & 0 & 2 & 0 & 6,626 & 0 & 1,363 \\
\hline LA & 0 & 0 & 0 & 0 & 0 & 2,614 & 0 & 2,967 \\
\hline MA & 79 & 62 & 1,920 & 879 & 1,920 & 2,068 & 2,793 & 957 \\
\hline MD & 0 & 34 & 0 & 605 & 111 & 4,449 & 266 & 2,468 \\
\hline ME & 409 & 0 & 450 & 0 & 2,948 & 843 & 1,764 & 338 \\
\hline $\mathrm{Ml}$ & 816 & 0 & 4,961 & 0 & 7,026 & 3,113 & 17,234 & 1,369 \\
\hline $\mathrm{MN}$ & 3,745 & 2 & 12,556 & 2 & 14,628 & 1,296 & 20,095 & 651 \\
\hline $\mathrm{MO}$ & 363 & 0 & 2,429 & 122 & 3,402 & 12,064 & 2,851 & 4,650 \\
\hline MS & 0 & 0 & 0 & 0 & 0 & 8,184 & 0 & 3,050 \\
\hline MT & 22 & 0 & 22 & 0 & 338 & 0 & 338 & 0 \\
\hline $\mathrm{NC}$ & 0 & 189 & 3,760 & 290 & 14,860 & 8,191 & 7,637 & 2,626 \\
\hline ND & 1,285 & 0 & 4,788 & 0 & 8,876 & 342 & 7,790 & 285 \\
\hline $\mathrm{NE}$ & 176 & 0 & 4,234 & 0 & 6,249 & 2,566 & 6,104 & 1,969 \\
\hline $\mathrm{NH}$ & 222 & 0 & 420 & 39 & 2,340 & 837 & 1,169 & 203 \\
\hline NJ & 9 & 277 & 5,423 & 1,447 & 8,423 & 4,423 & 6,423 & 1,566 \\
\hline NM & 176 & 51 & 1,671 & 51 & 4,071 & 1,949 & 4,071 & 2,749 \\
\hline NY & 1,259 & 55 & 9,799 & 69 & 10,355 & 6,798 & 11,622 & 3,302 \\
\hline $\mathrm{OH}$ & 9 & 43 & 3,151 & 380 & 5,668 & 3,654 & 7,545 & 1,833 \\
\hline OK & 2,484 & 0 & 8,974 & 0 & 14,006 & 1,682 & 22,967 & 2,309 \\
\hline PA & 969 & 46 & 1,175 & 835 & 5,037 & 3,584 & 5,348 & 1,831 \\
\hline $\mathrm{RI}$ & 0 & 0 & 127 & 0 & 127 & 564 & 242 & 249 \\
\hline SC & 0 & 0 & 0 & 0 & 0 & 14,763 & 0 & 5,141 \\
\hline SD & 1,253 & 0 & 7,131 & 0 & 8,879 & 2,203 & 12,017 & 1,020 \\
\hline TN & 20 & 11 & 100 & 11 & 363 & 15,046 & 1,048 & 3,656 \\
\hline$T X$ & 744 & 0 & 5,159 & 0 & 5,159 & 1,917 & 5,159 & 2,640 \\
\hline $\mathrm{VA}$ & 97 & 0 & 1,404 & 10 & 6,705 & 10,362 & 4,804 & 4,658 \\
\hline$\overline{V T}$ & 59 & 19 & 207 & 19 & 1,701 & 103 & 1,151 & 41 \\
\hline WI & 476 & 4 & 1,814 & 4 & 1,934 & 1,094 & 3,529 & 549 \\
\hline WV & 625 & 10 & 2,113 & 13 & 2,857 & 621 & 2,857 & 311 \\
\hline Total & 23,778 & 970 & 104,299 & 5,016 & 183,191 & 218,687 & 222,206 & 109,806 \\
\hline
\end{tabular}




\section{Appendix D: Regional Dispatch Stacks}

Regional dispatch stacks for the periods of high load conditions, high VG conditions and the highest net-load ramp.

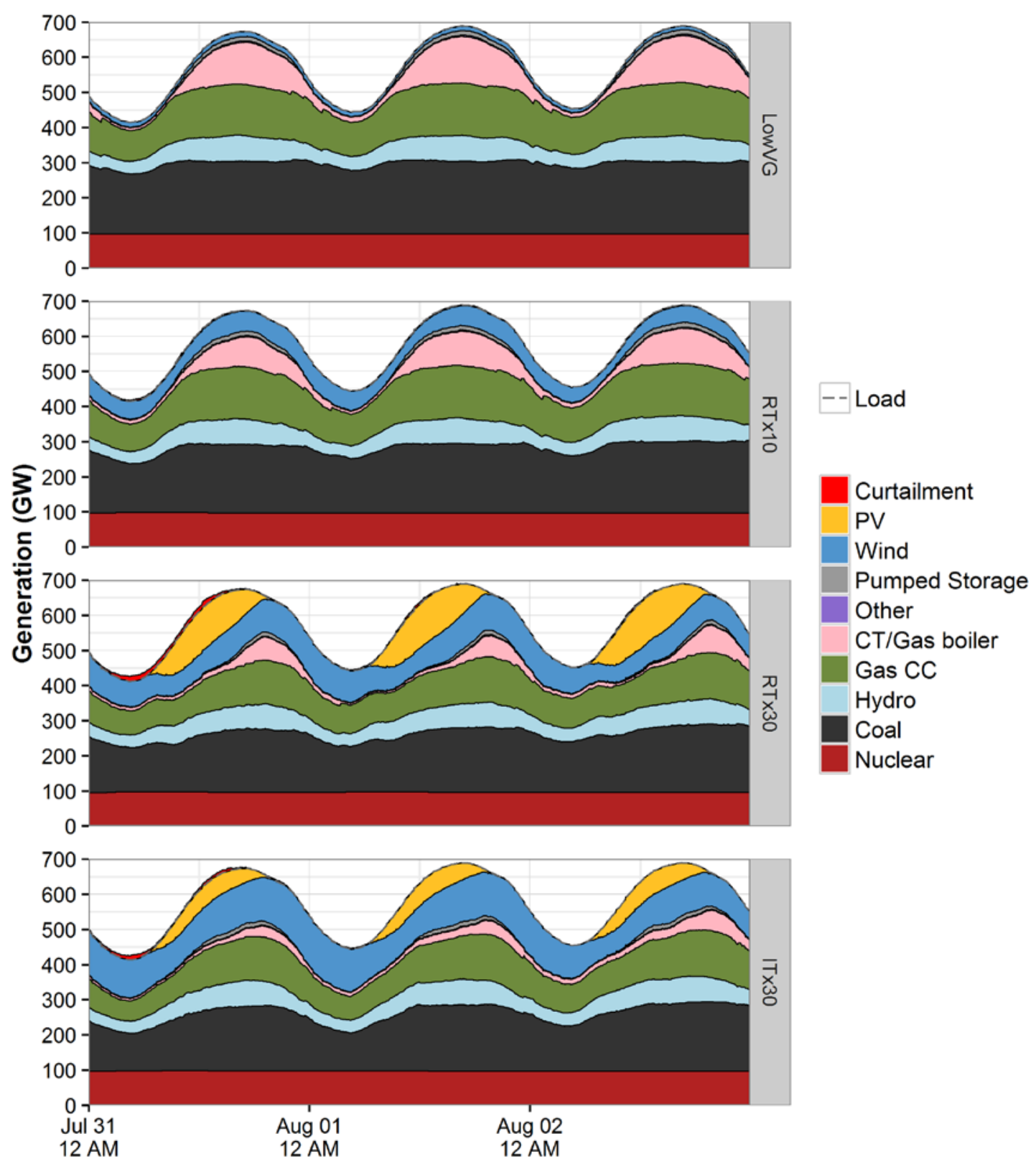

Figure 106. US E.I. generation during peak load period for all scenarios 

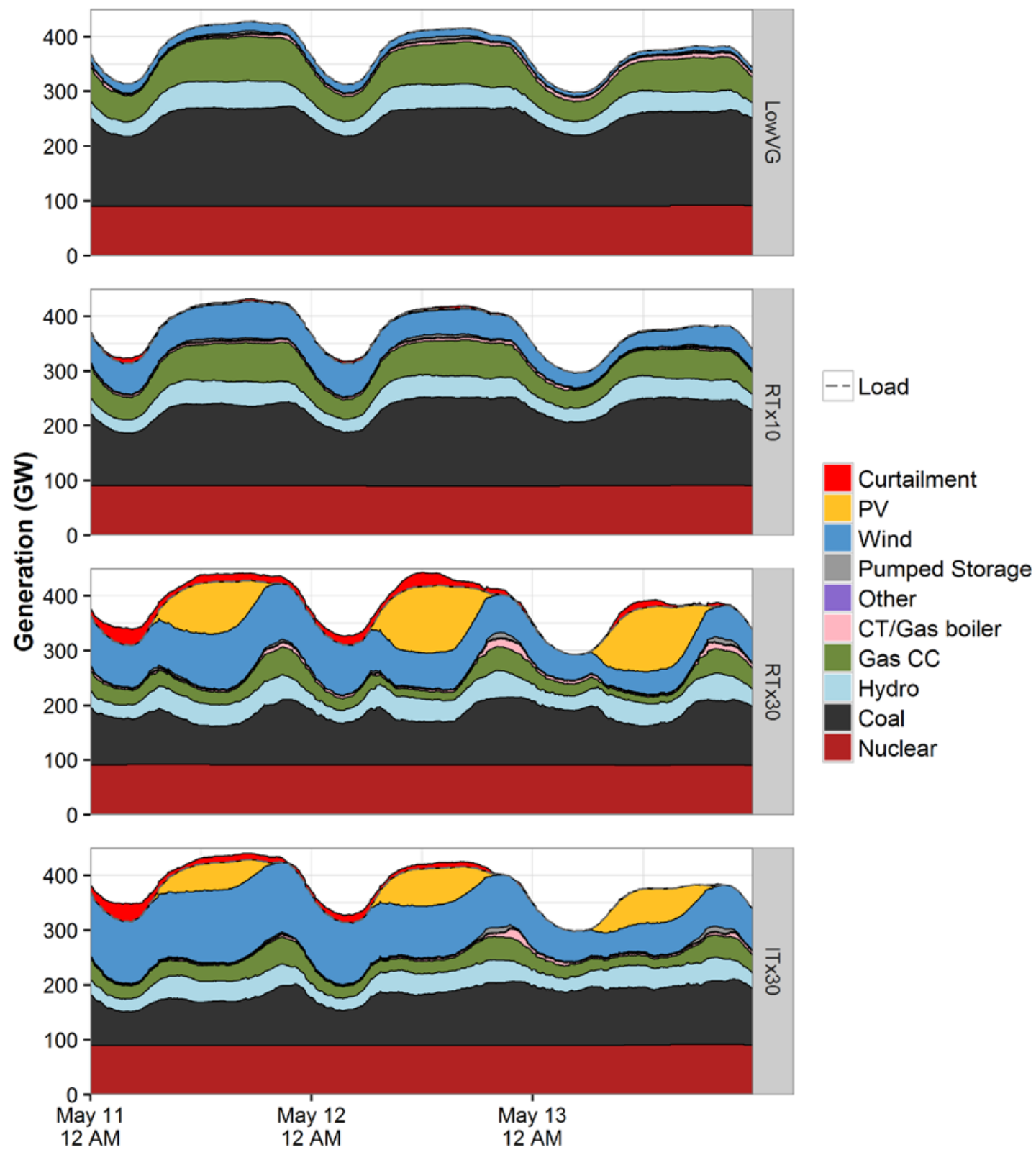

Figure 107. US E.I. generation during high VG period for all scenarios 

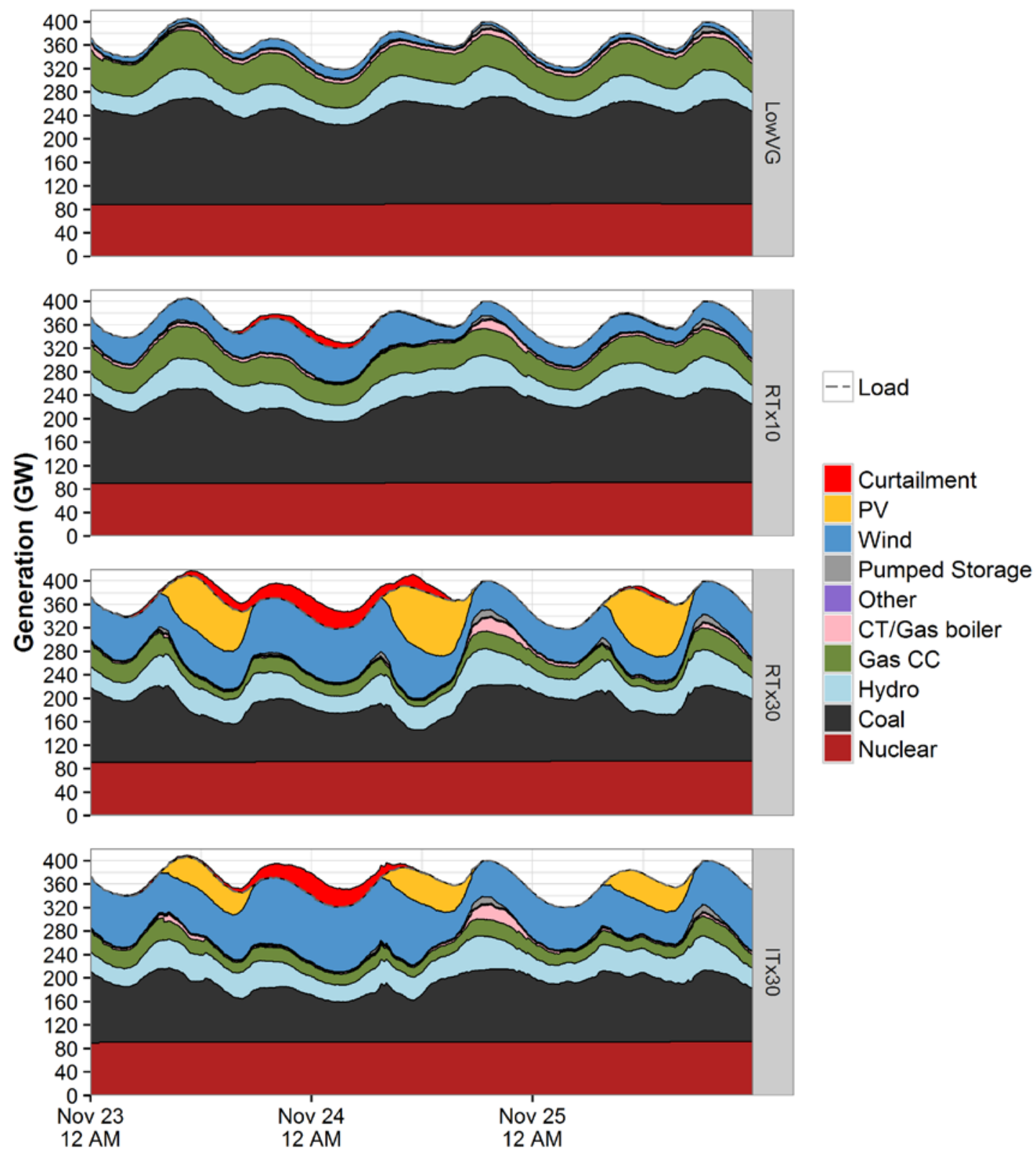

Figure 108. US E.I. generation during the highest net load ramp for all scenarios 


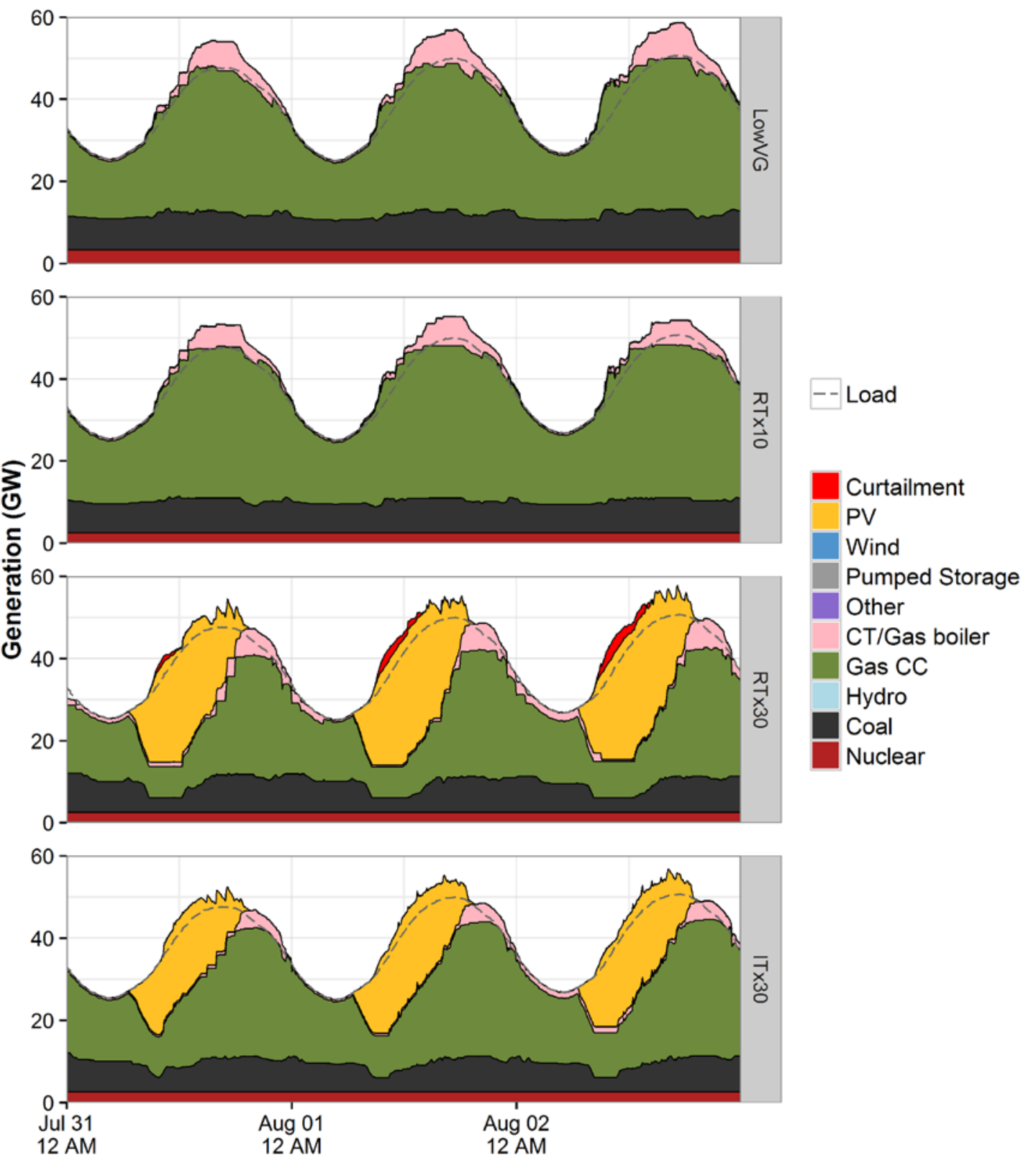

Figure 109. FRCC generation during peak load period for all scenarios 

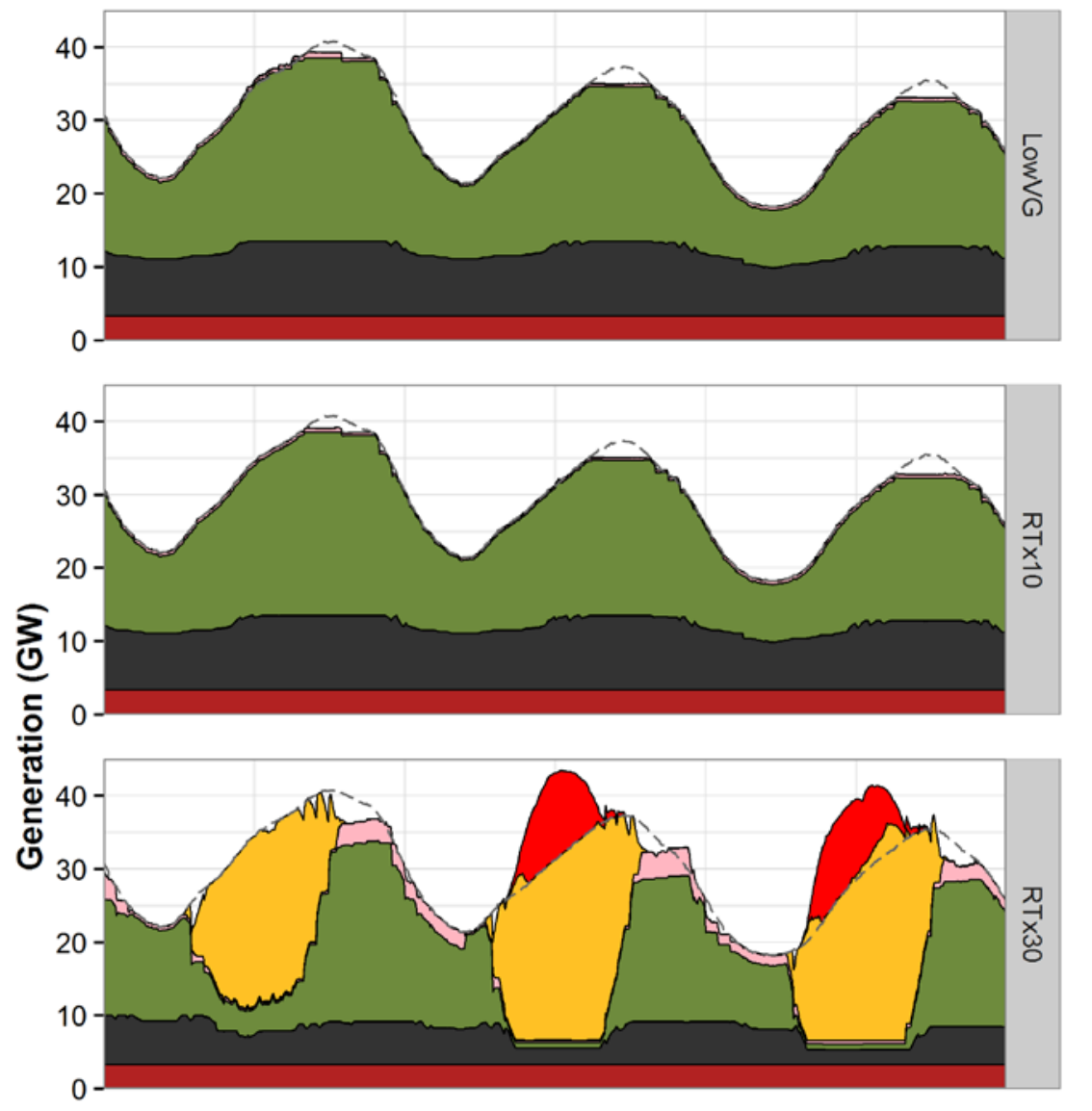

- Load

Curtailment

PV

Wind

Pumped Storage

Other

CT/Gas boiler

Gas CC

Hydro

Coal

Nuclear

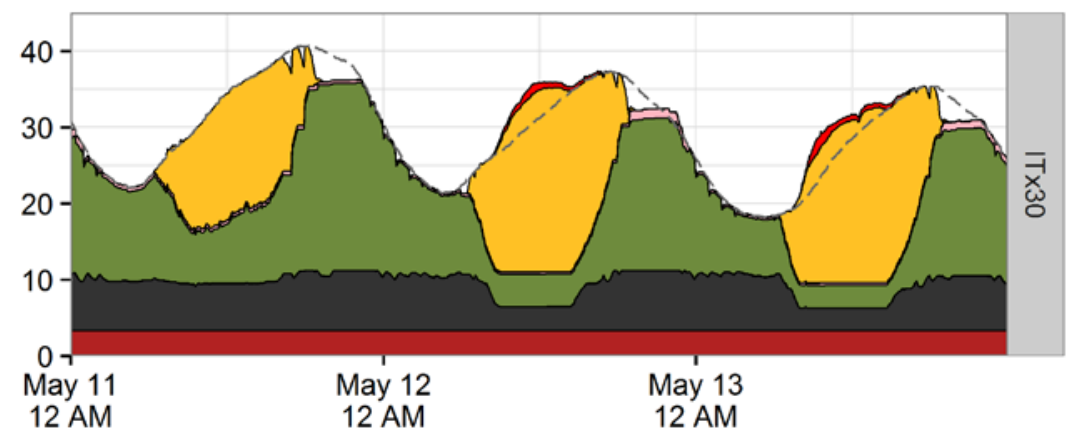

Figure 110. FRCC generation during the high VG period for all scenarios 

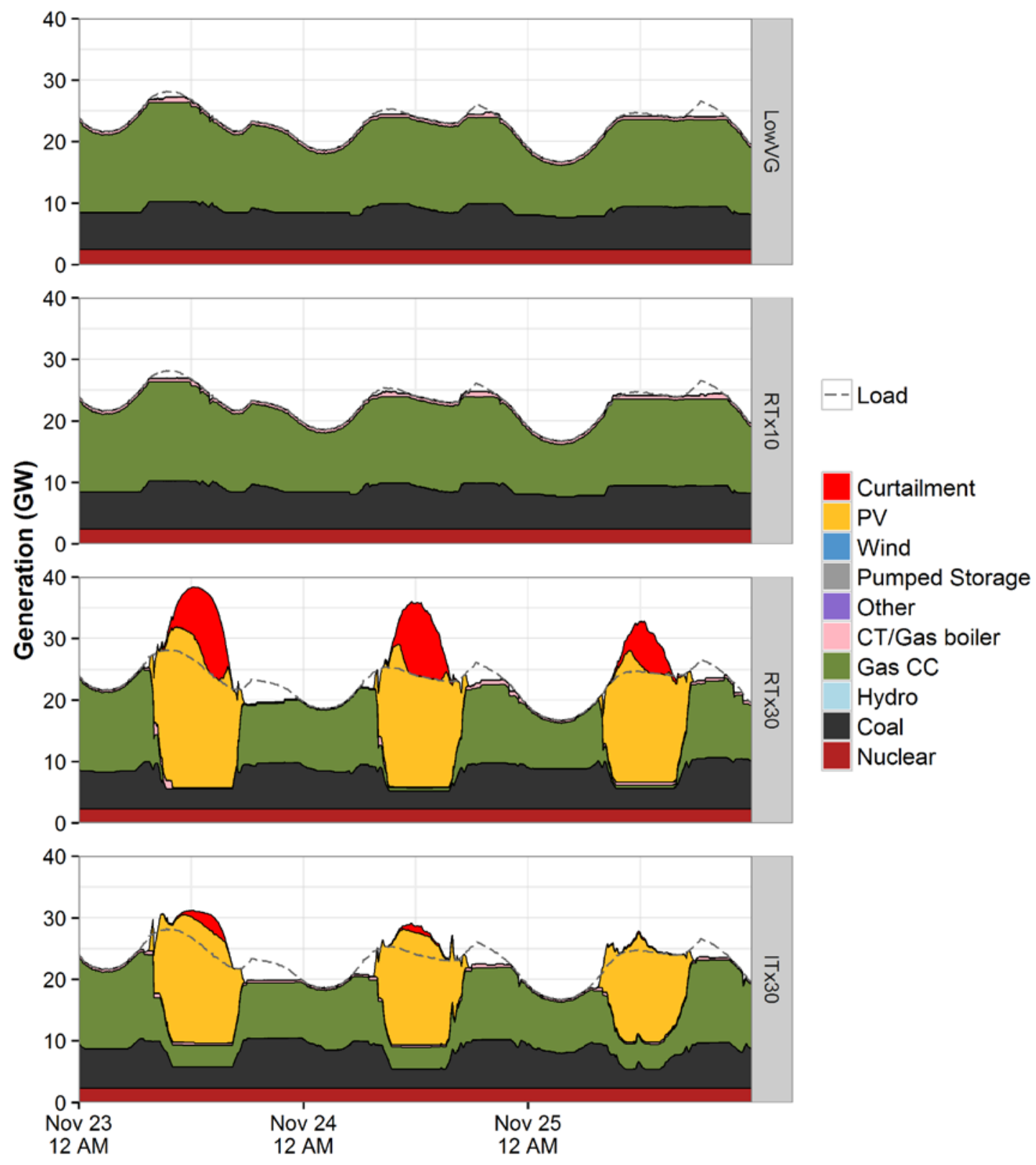

Figure 111. FRCC generation during the highest net load ramp for all scenarios 


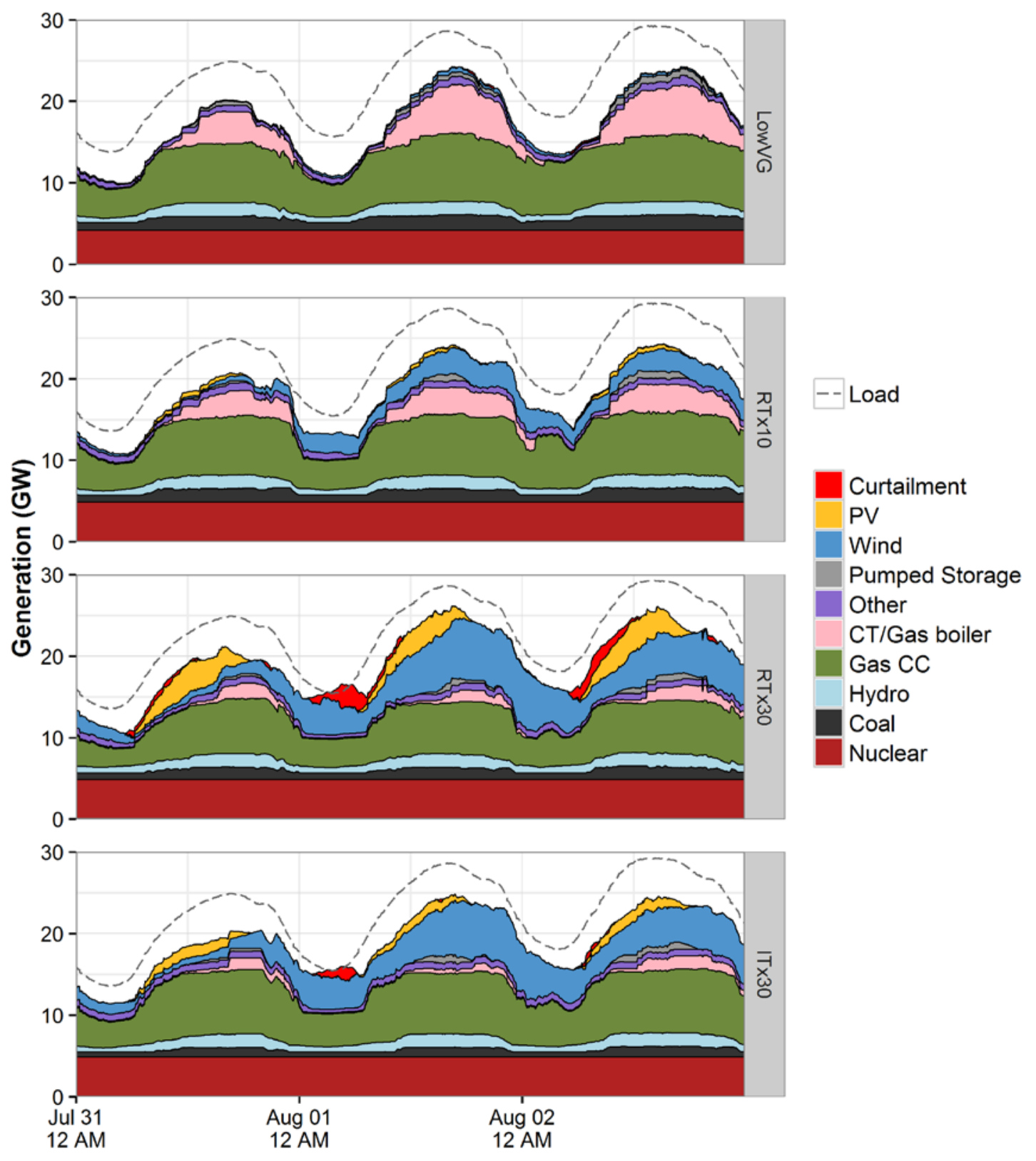

Figure 112. ISO-NE generation during peak load period for all scenarios 


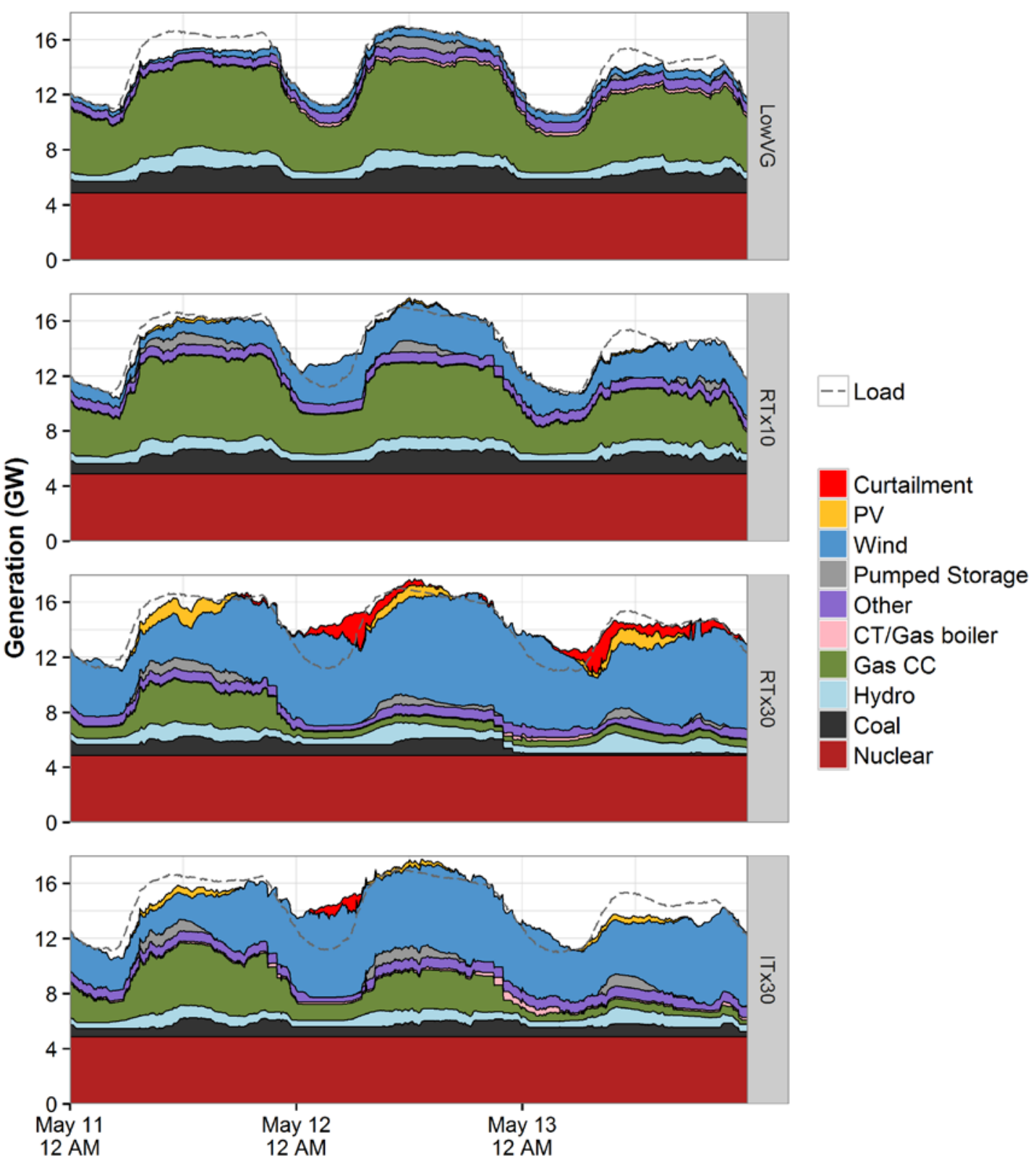

Figure 113. ISO-NE generation during high VG period for all scenarios 


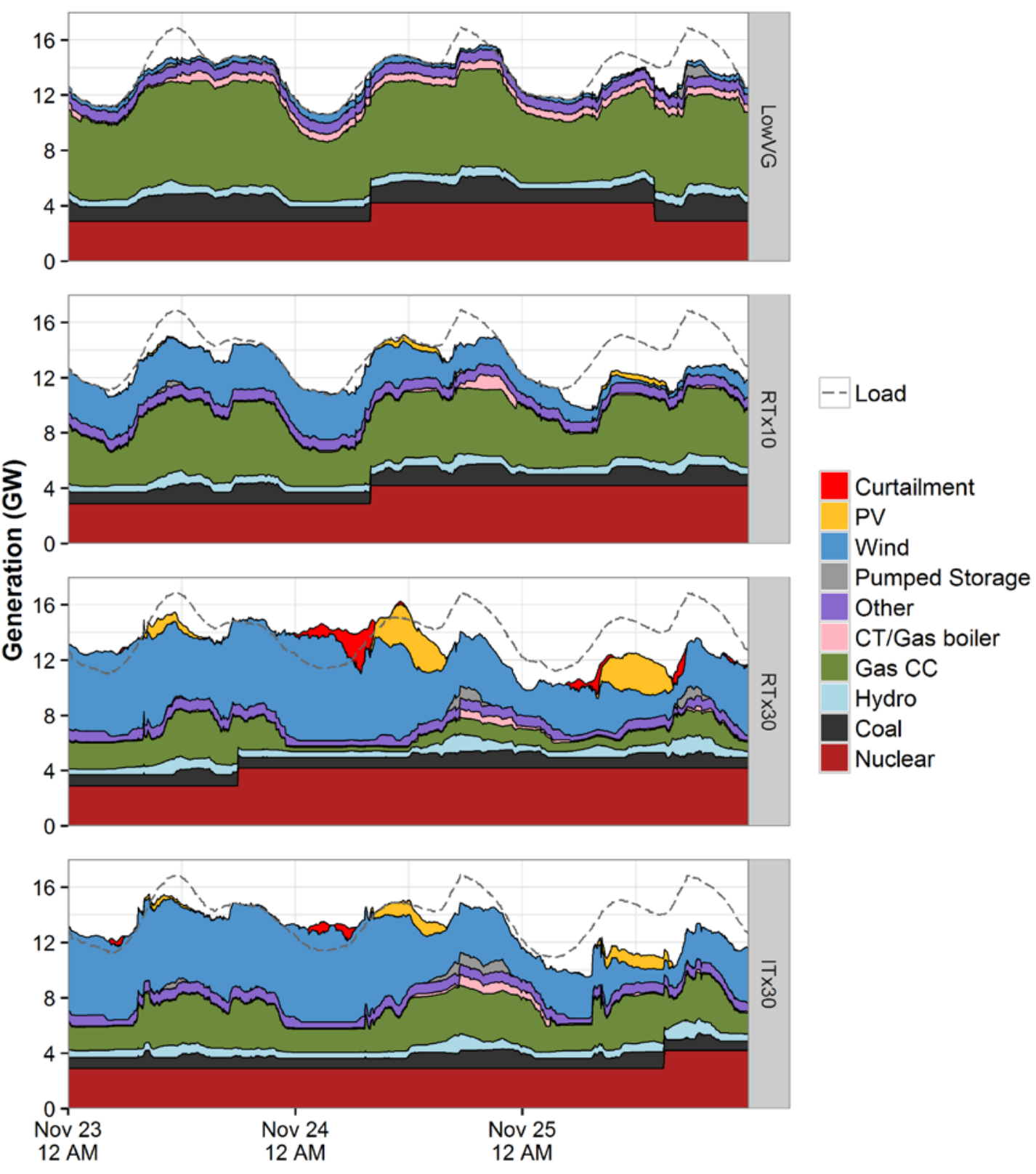

Figure 114. ISO-NE generation during the highest net load ramp for all scenarios 

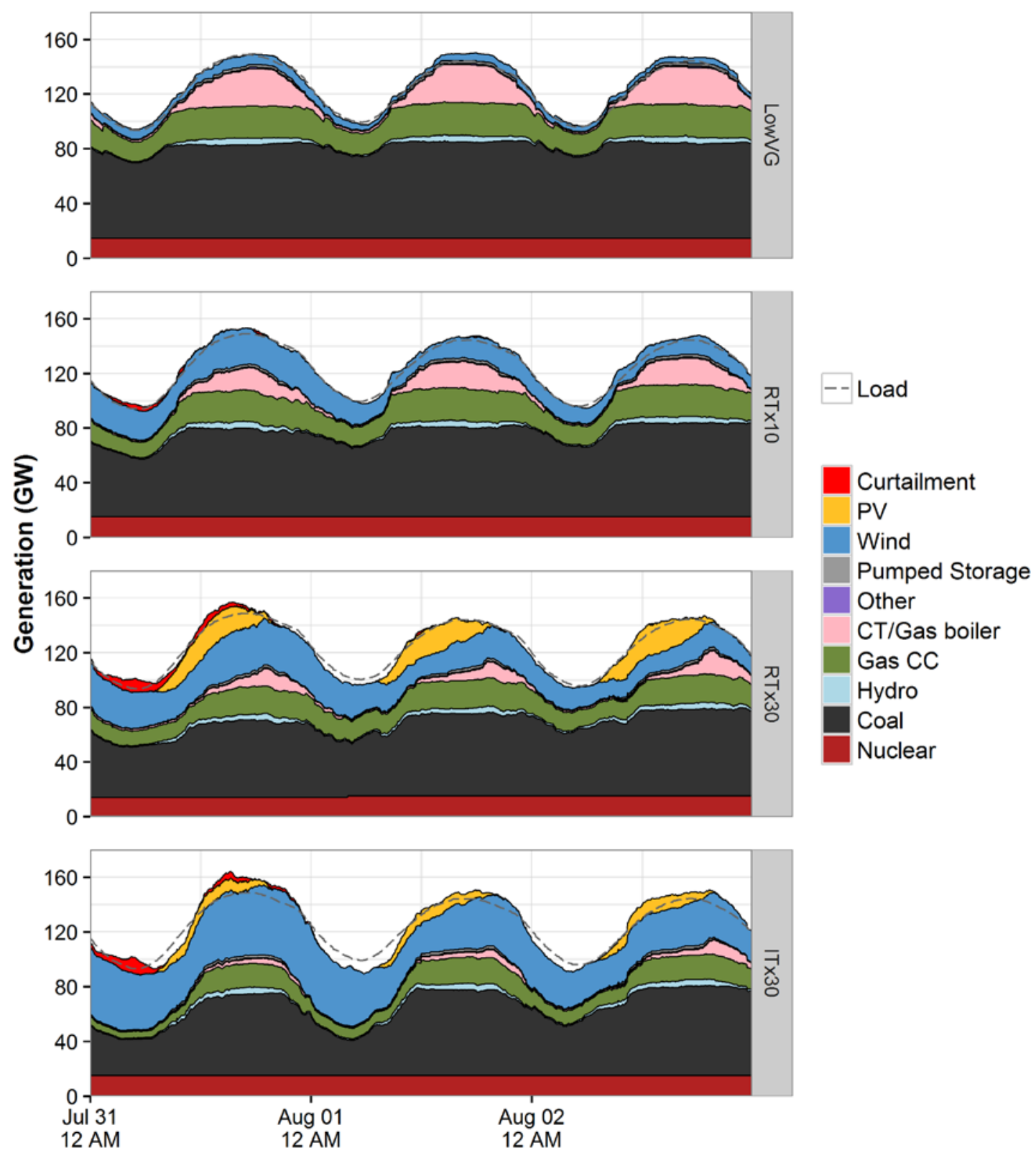

Figure 115. MISO generation during peak load period for all scenarios 

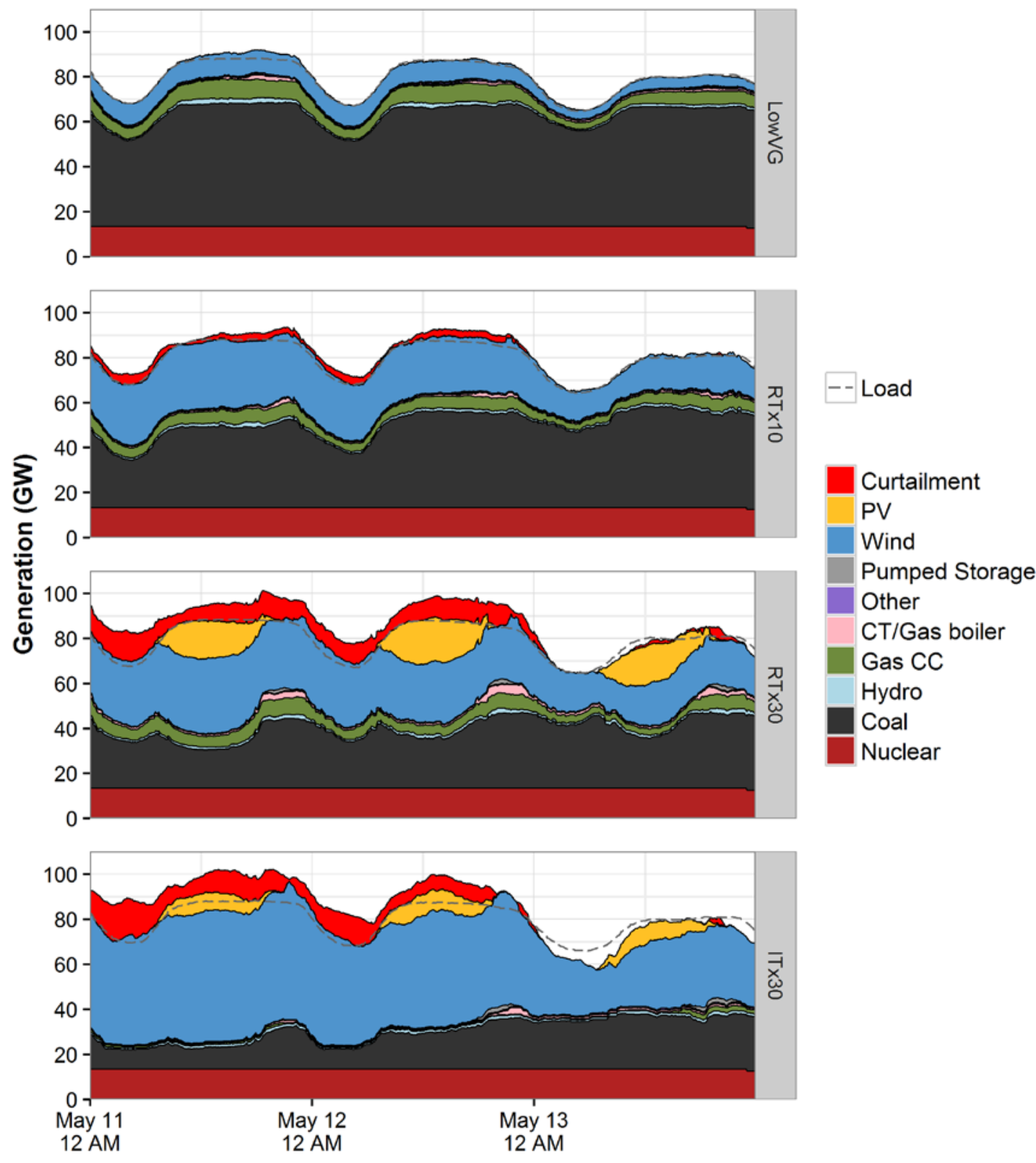

Figure 116. MISO generation during high VG period for all scenarios 


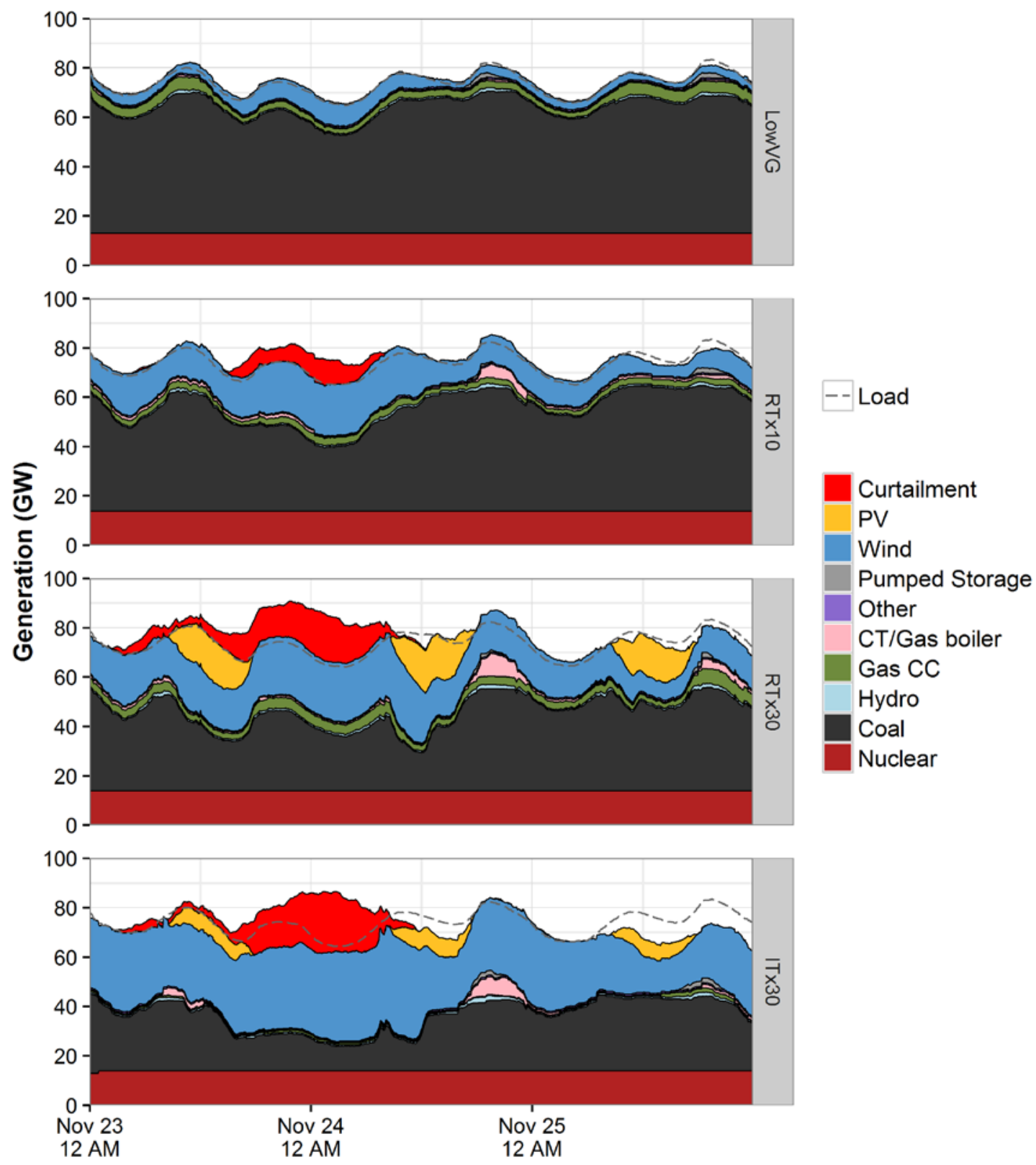

Figure 117. MISO generation during the highest net load ramp for all scenarios 


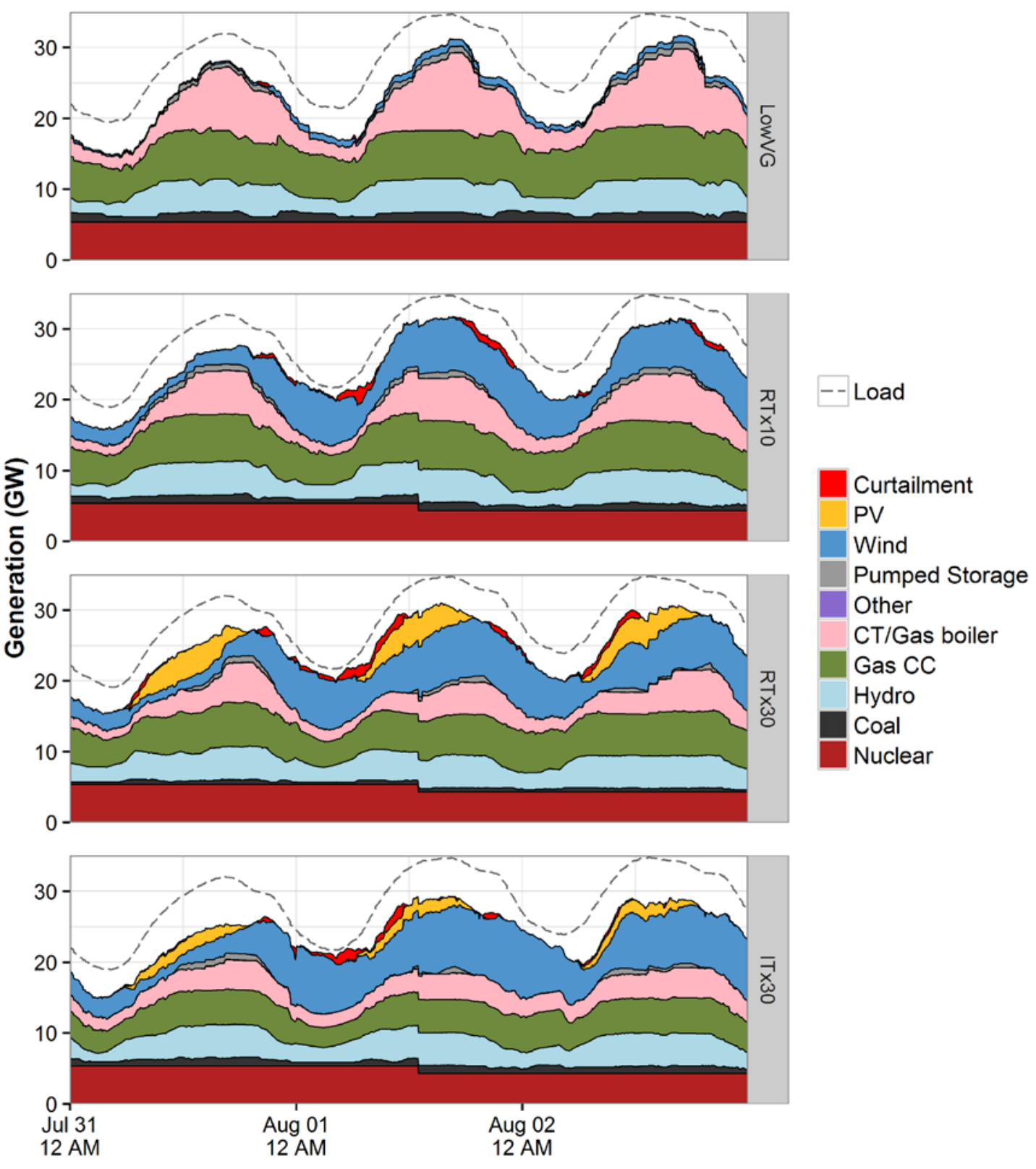

Figure 118. NYISO generation during peak load period for all scenarios 


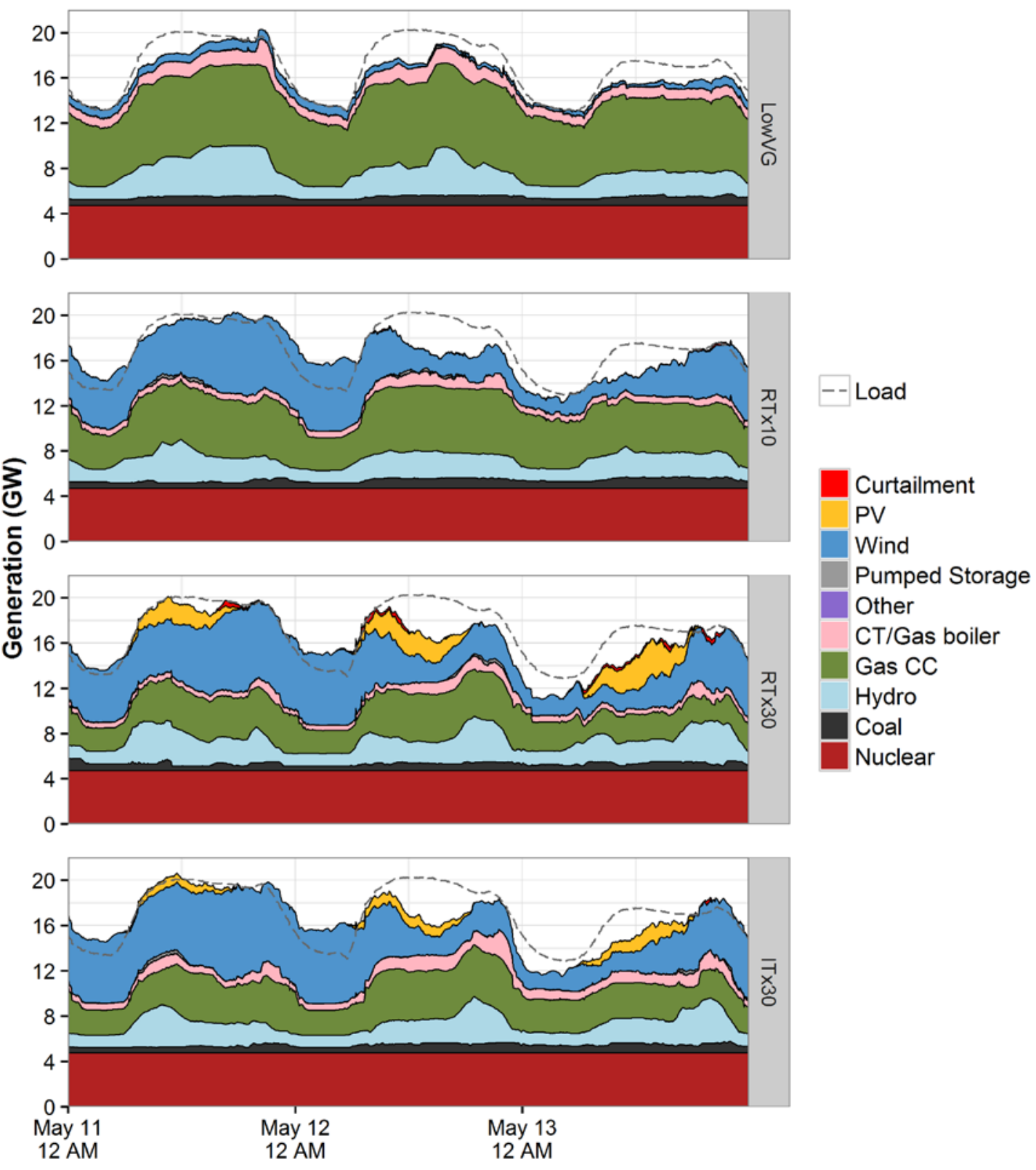

Figure 119. NYISO generation during high VG for all scenarios 


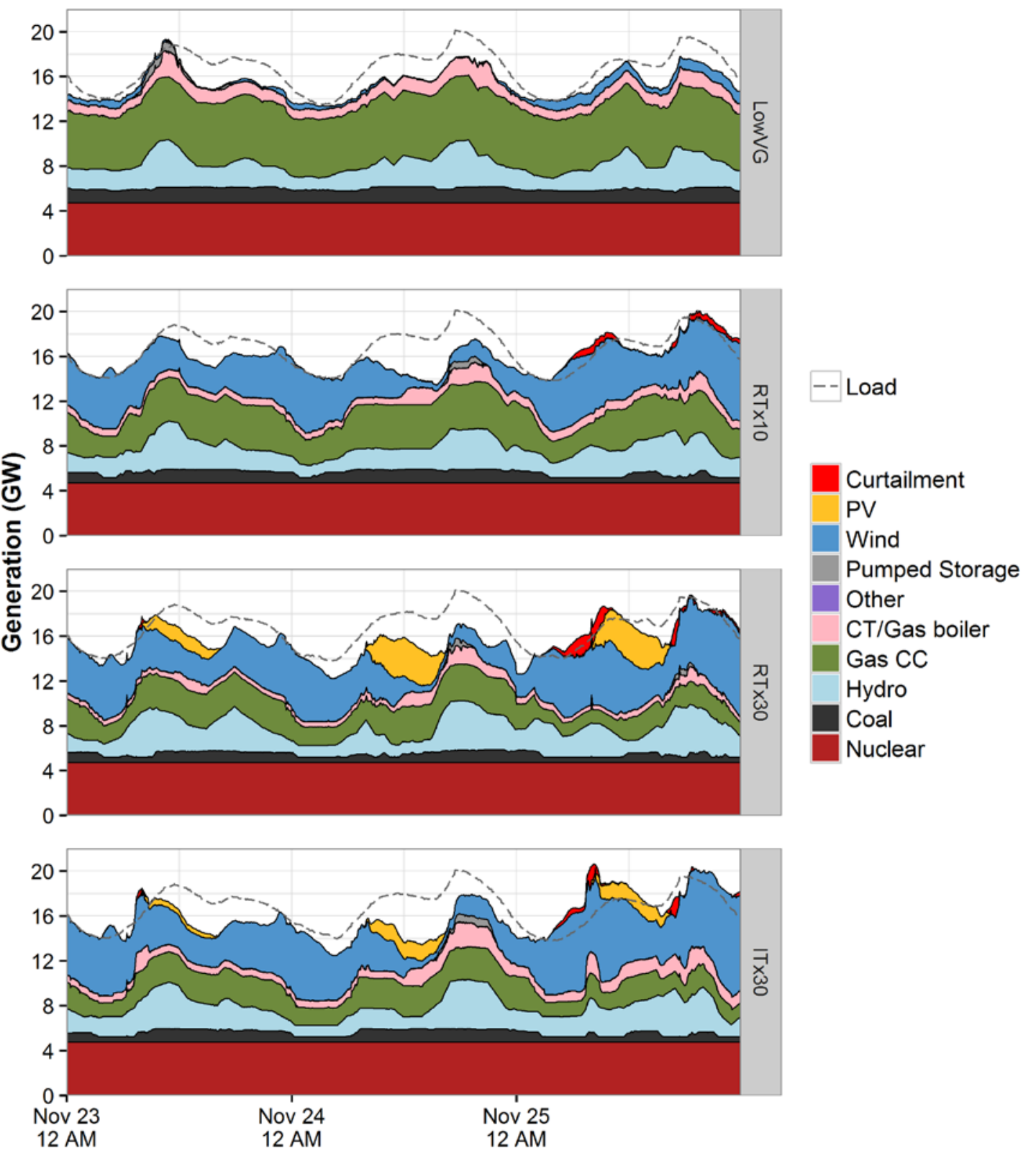

Figure 120. NYISO generation during the highest net load ramp for all scenarios 


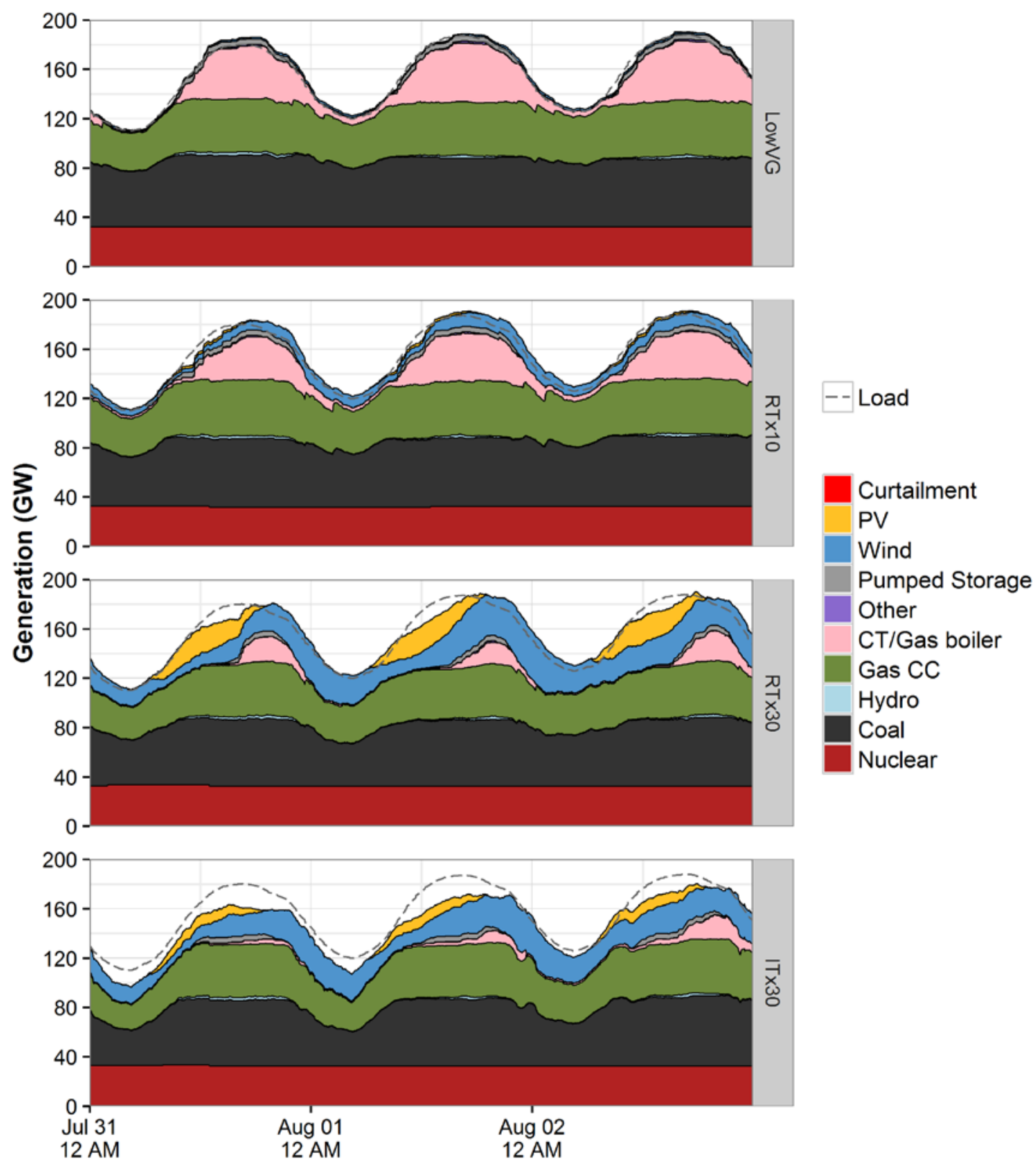

Figure 121. PJM generation during peak load period for all scenarios 

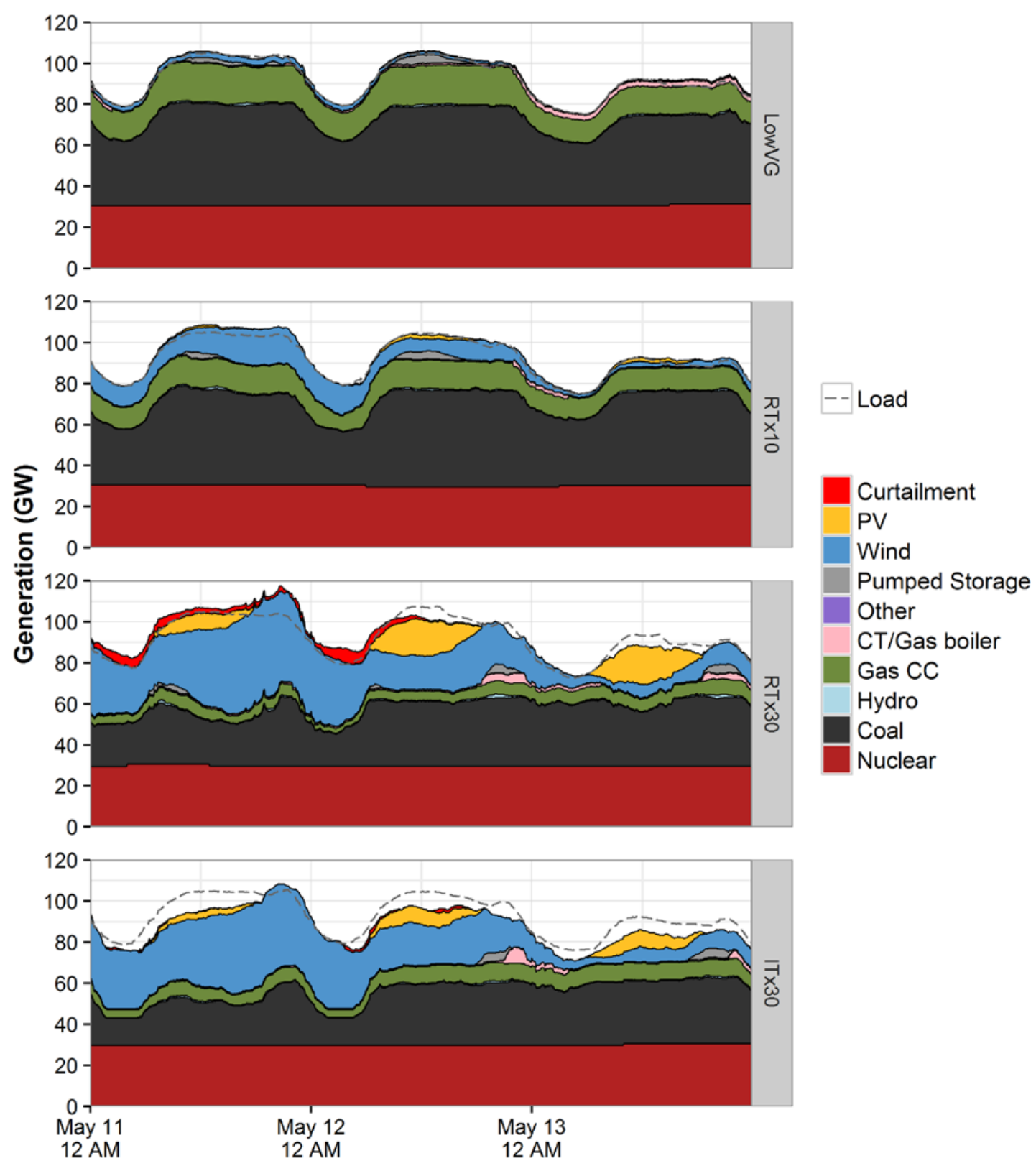

Figure 122. PJM generation during high VG for all scenarios 

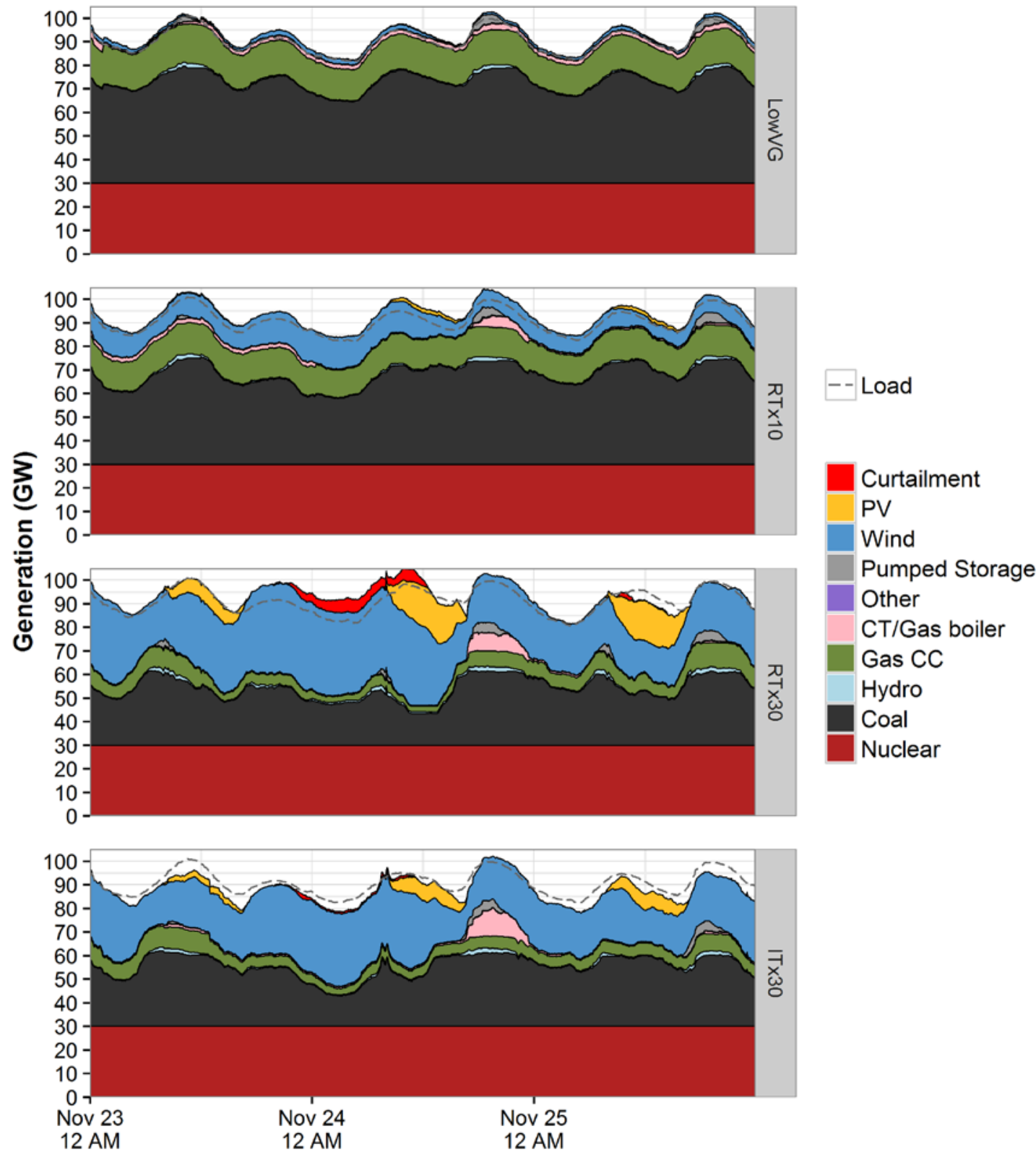

Figure 123. PJM generation during the highest net load ramp for all scenarios 


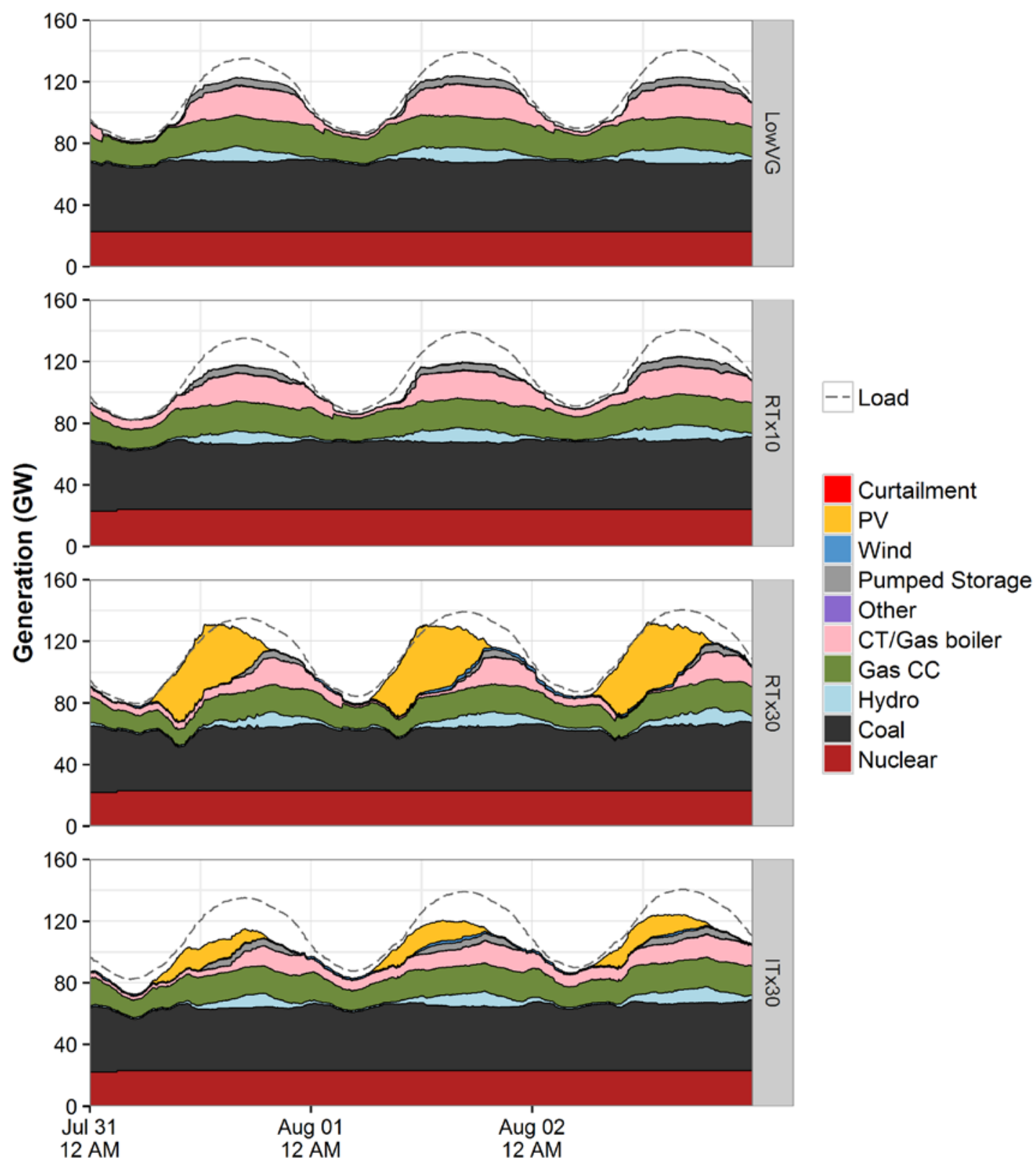

Figure 124. SERC generation during peak load period for all scenarios 


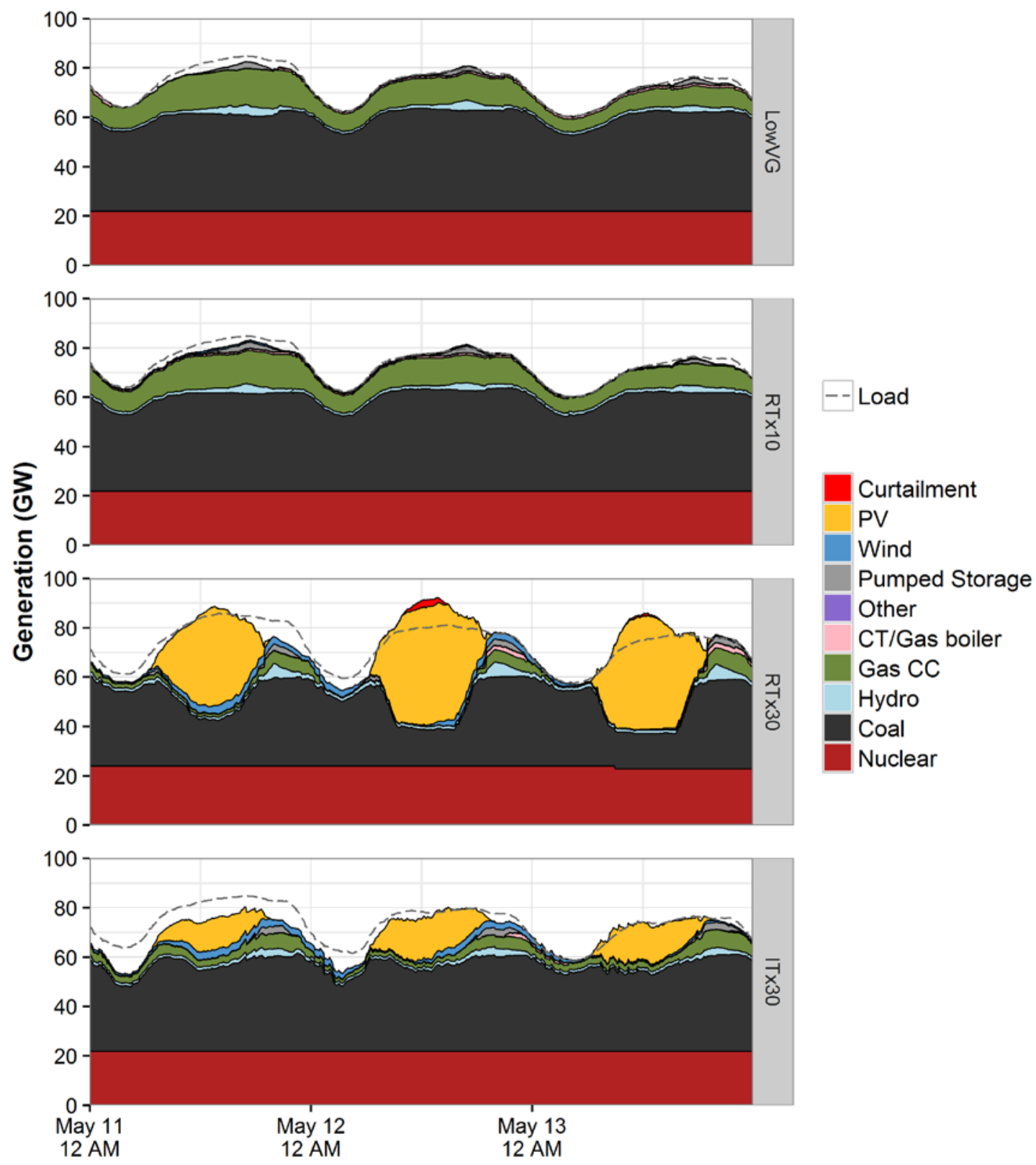

Figure 125. SERC generation during high VG for all scenarios 


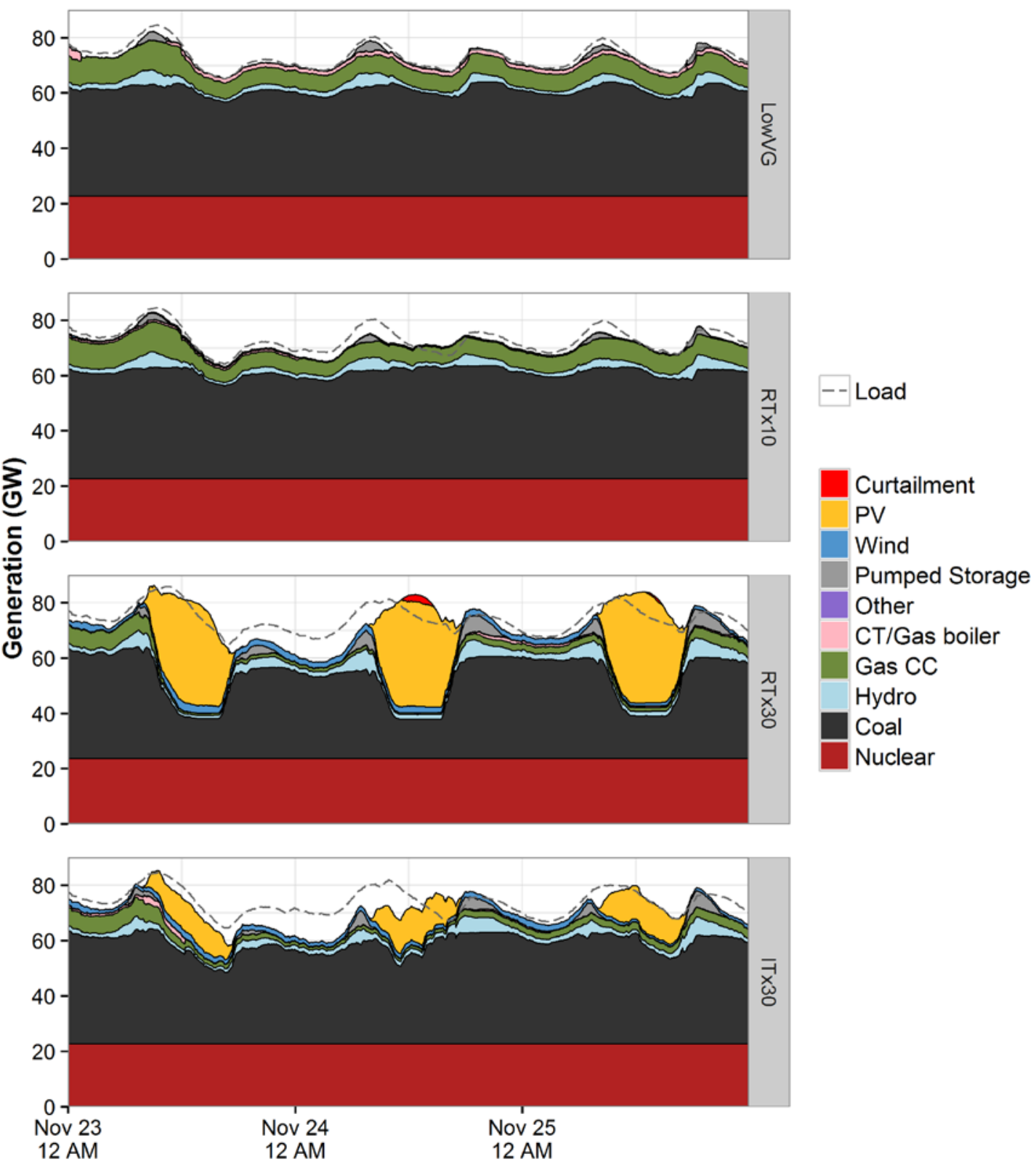

Figure 126. SERC generation during the highest net load ramp for all scenarios 

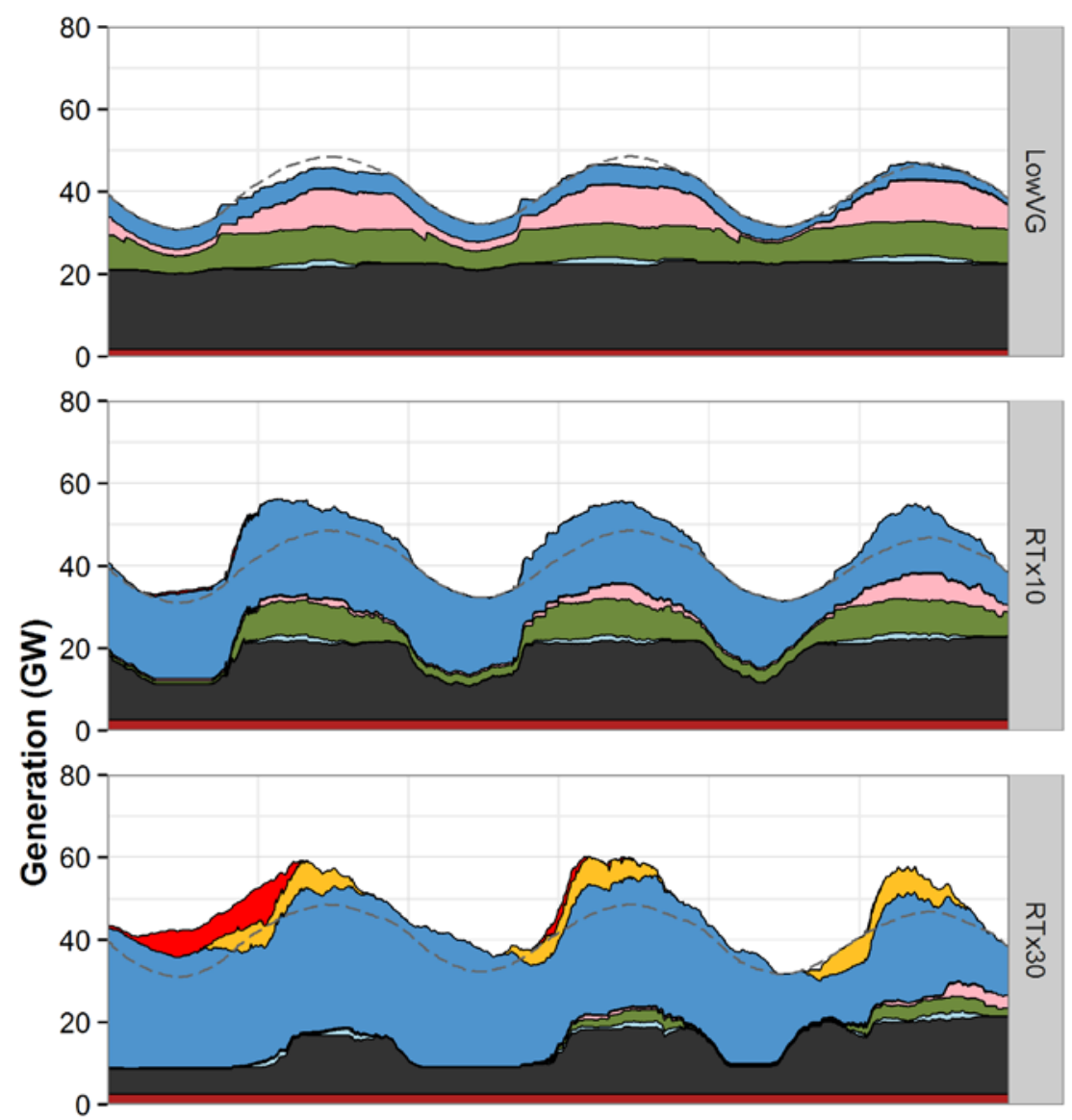

- Load

Curtailment

PV

Wind

Pumped Storage

Other

CT/Gas boiler

Gas CC

Hydro

Coal

Nuclear

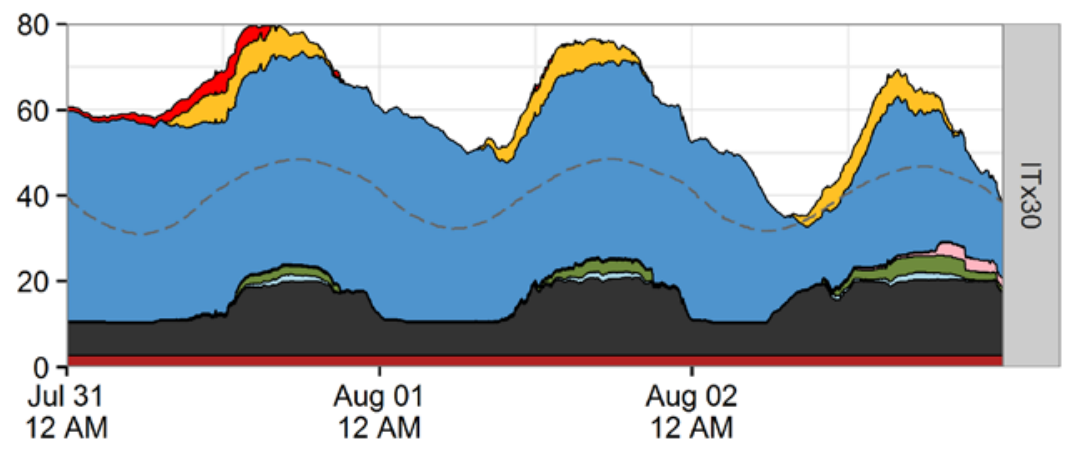

Figure 127. SPP generation during peak load period for all scenarios 


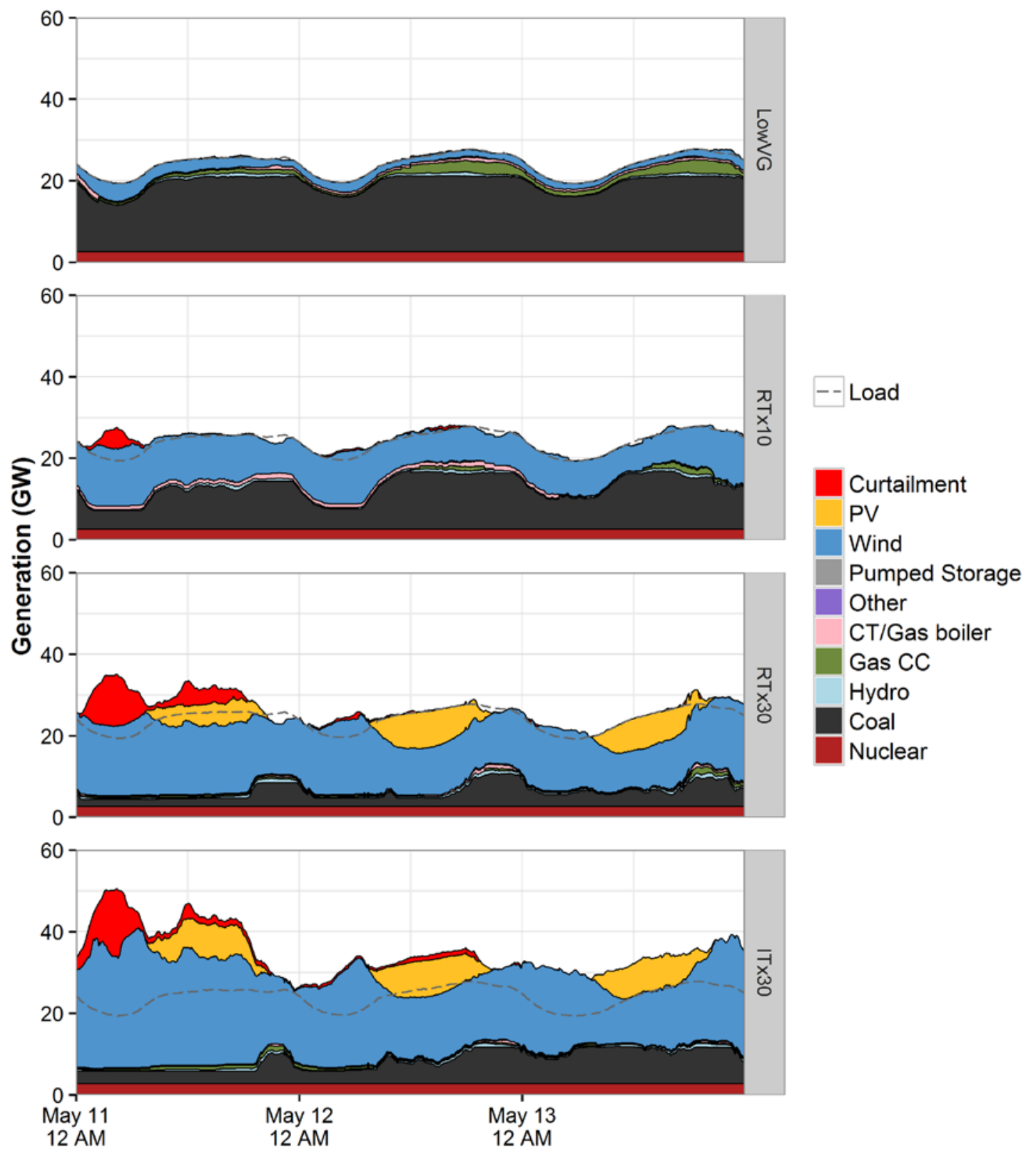

Figure 128. SPP generation during high VG for all scenarios 

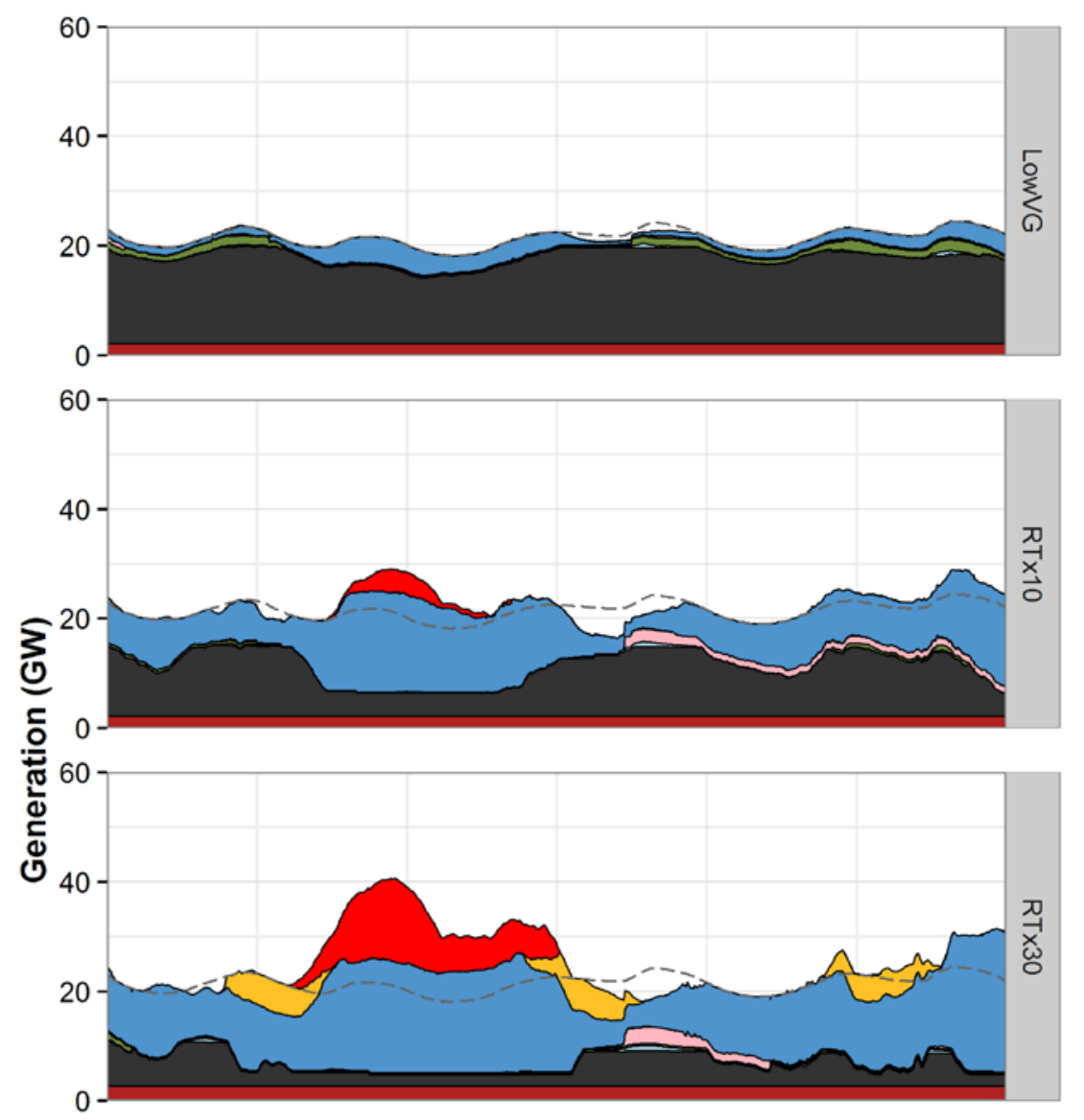

- Load

Curtailment

PV

Wind

Pumped Storage

Other

CT/Gas boiler

Gas CC

Hydro

Coal

Nuclear

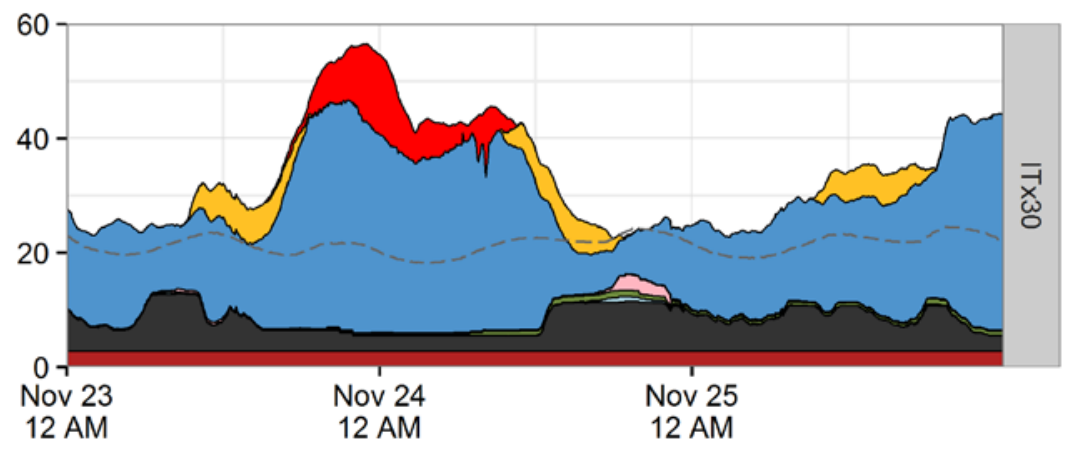

Figure 129. SPP generation during the highest net load ramp for all scenarios 


\section{Appendix E: Regional real-time and day-ahead Commitment and Dispatch}

Regional real-time and day-ahead unit commitment and dispatch.

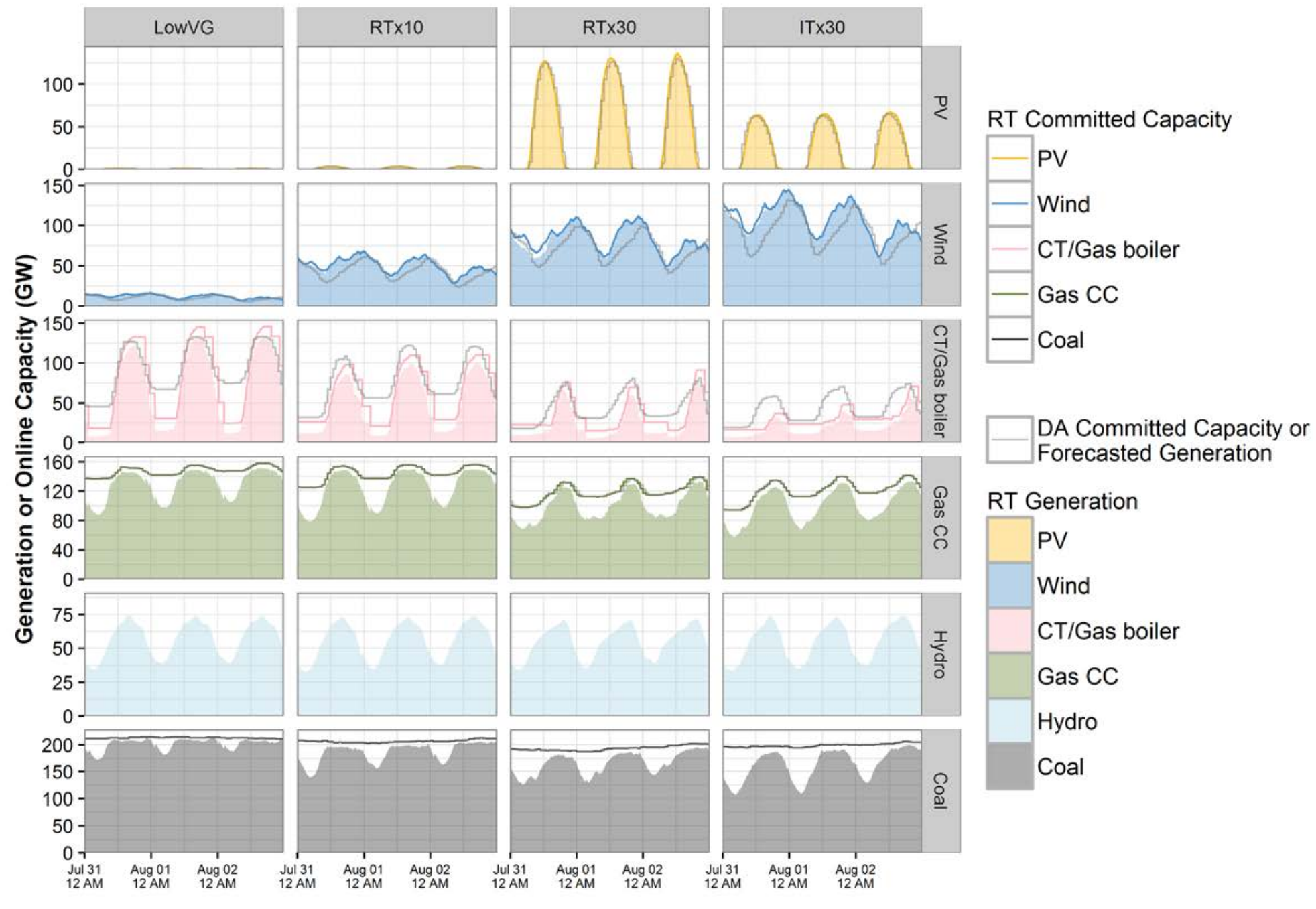

Figure 130. US E.I. generation during peak load period for all scenarios 


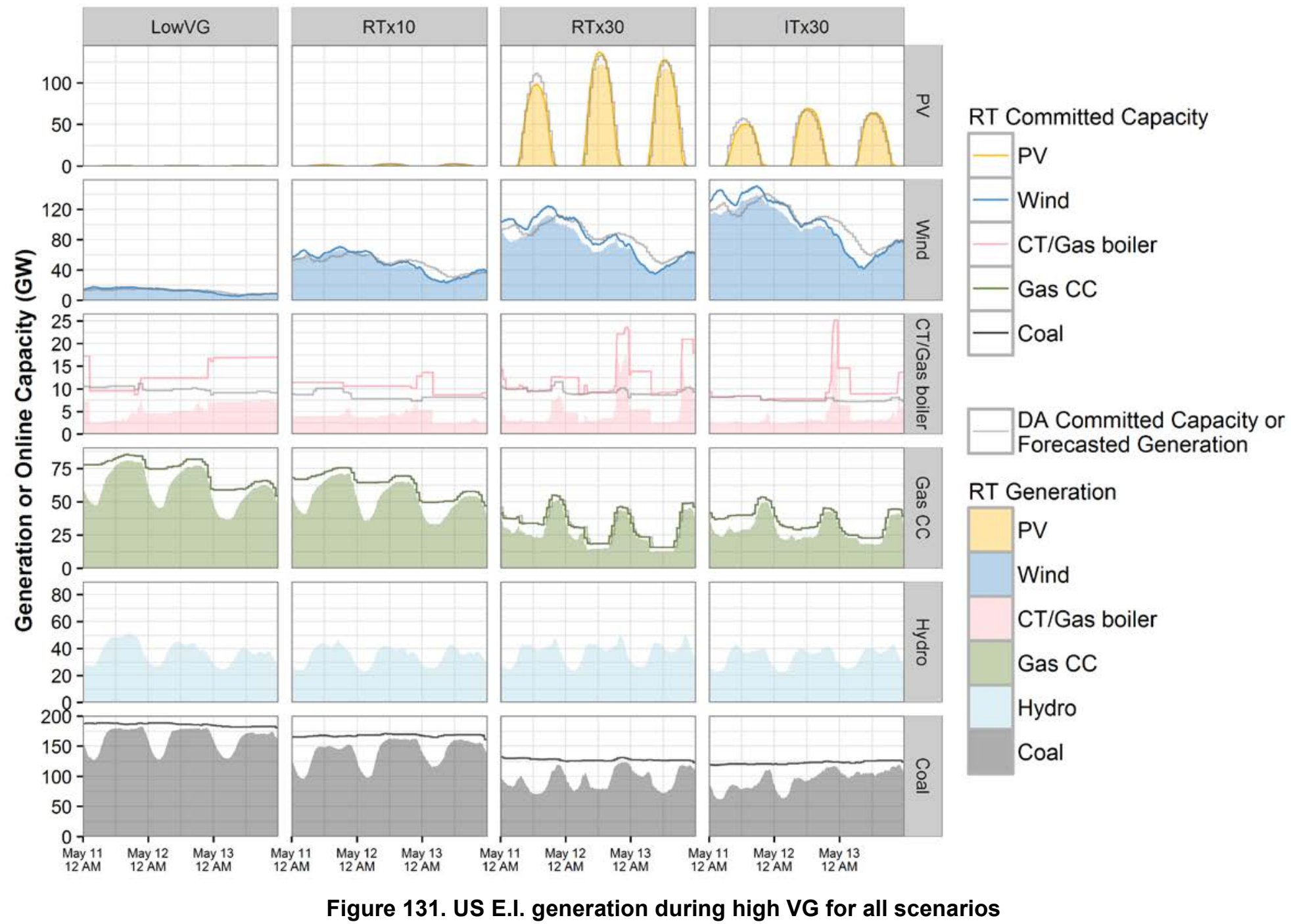




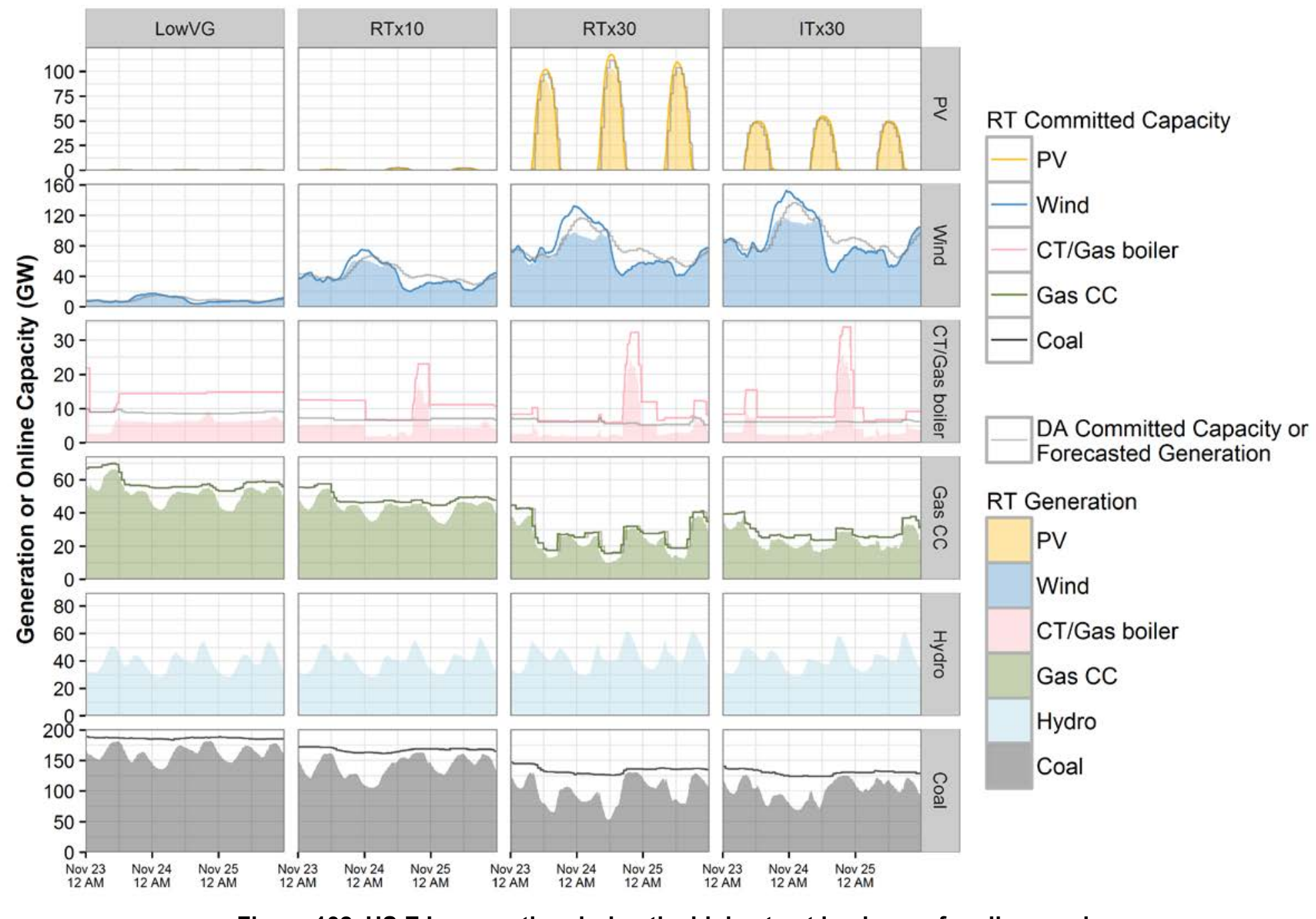

Figure 132. US E.I. generation during the highest net load ramp for all scenarios 




Figure 133. FRCC generation during peak load period for all scenarios 


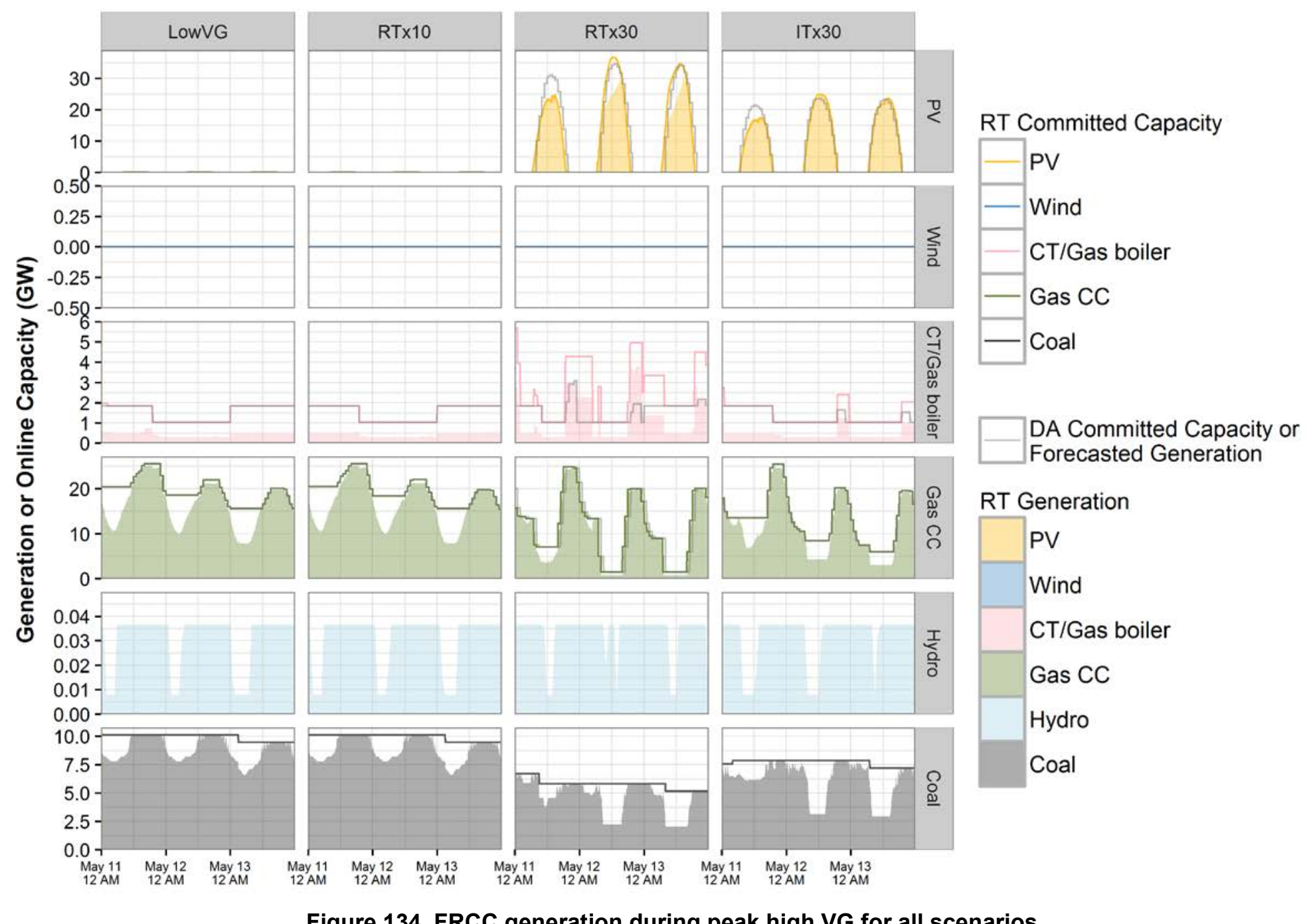

Figure 134. FRCC generation during peak high VG for all scenarios 


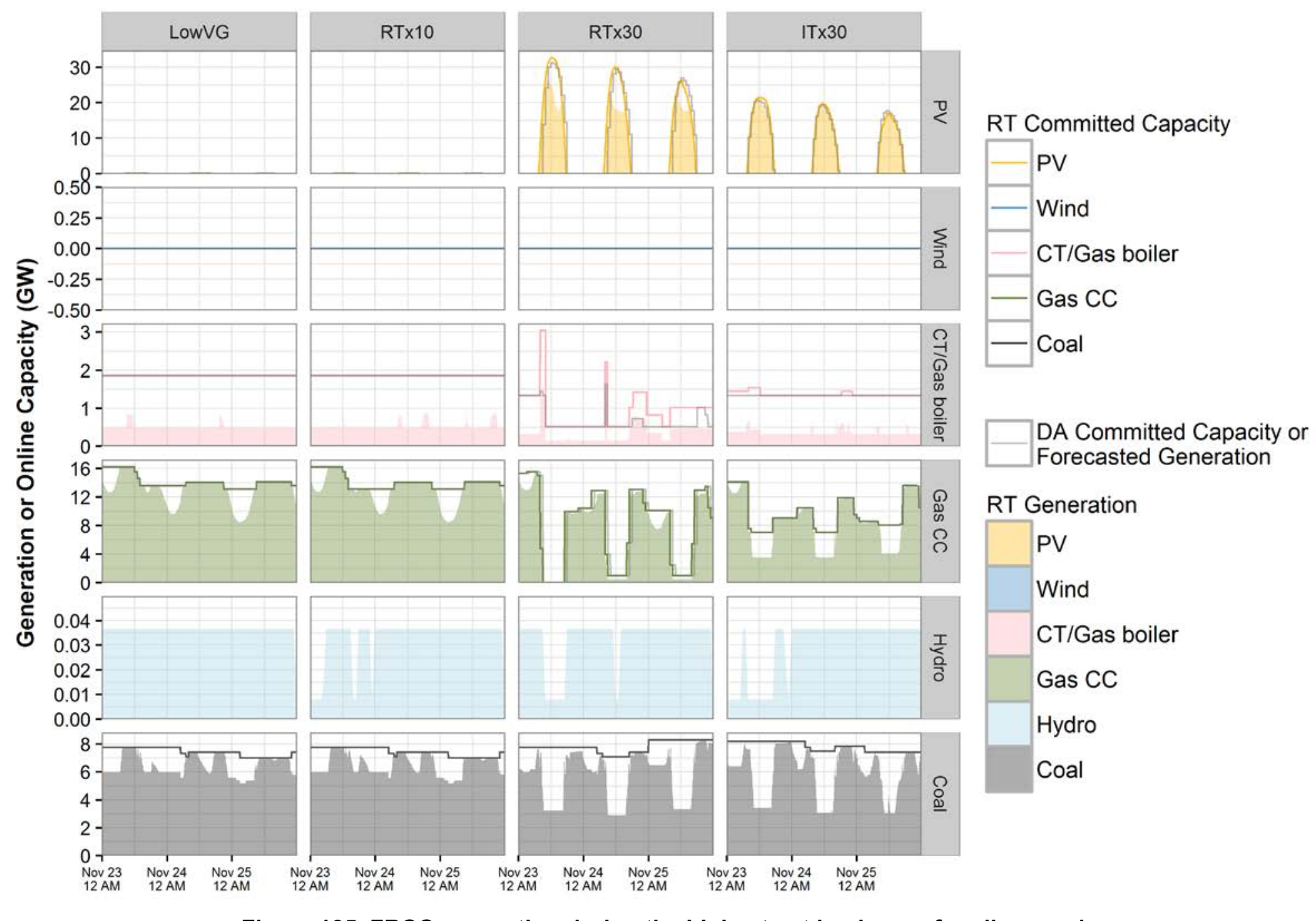

Figure 135. FRCC generation during the highest net load ramp for all scenarios 


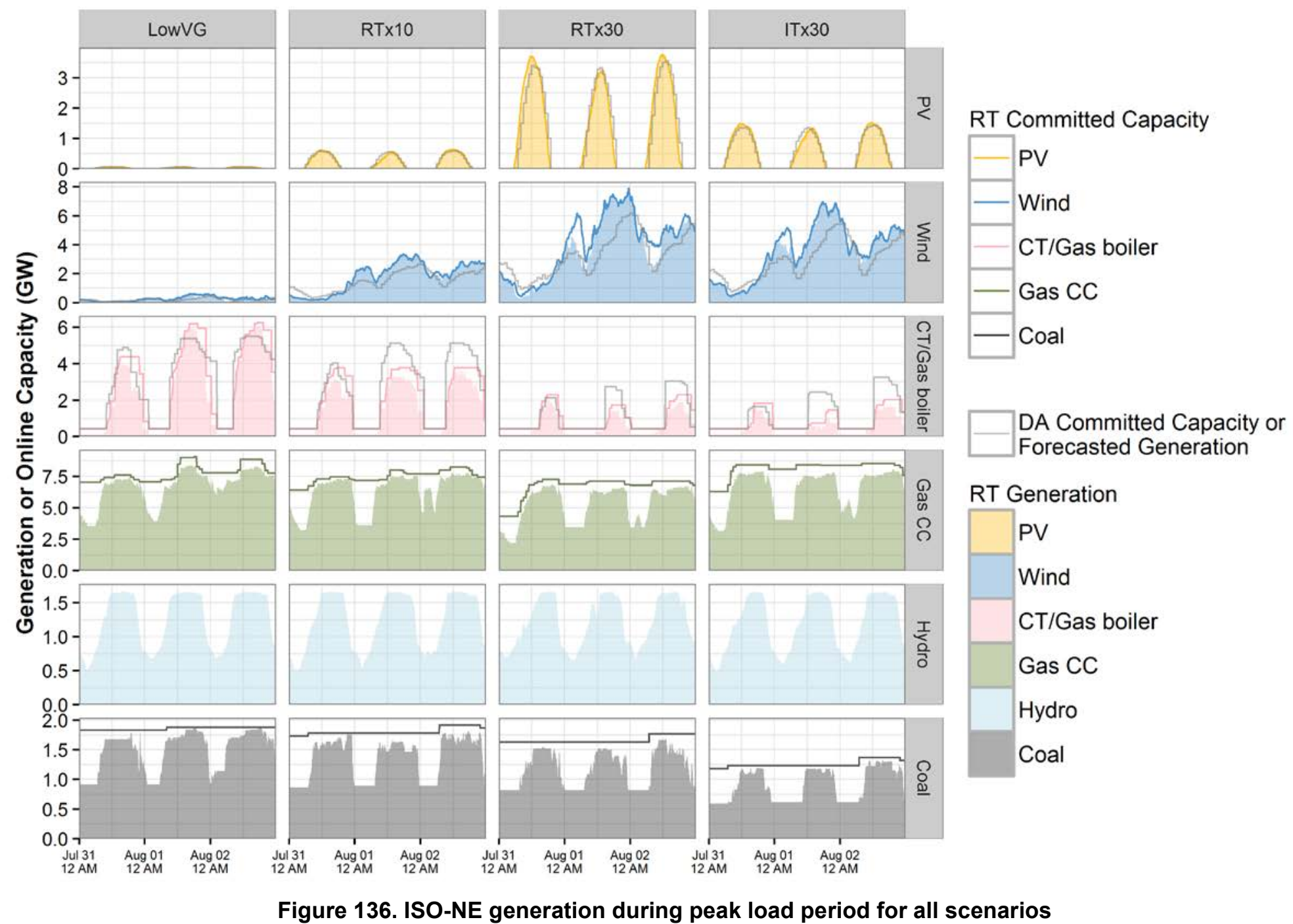




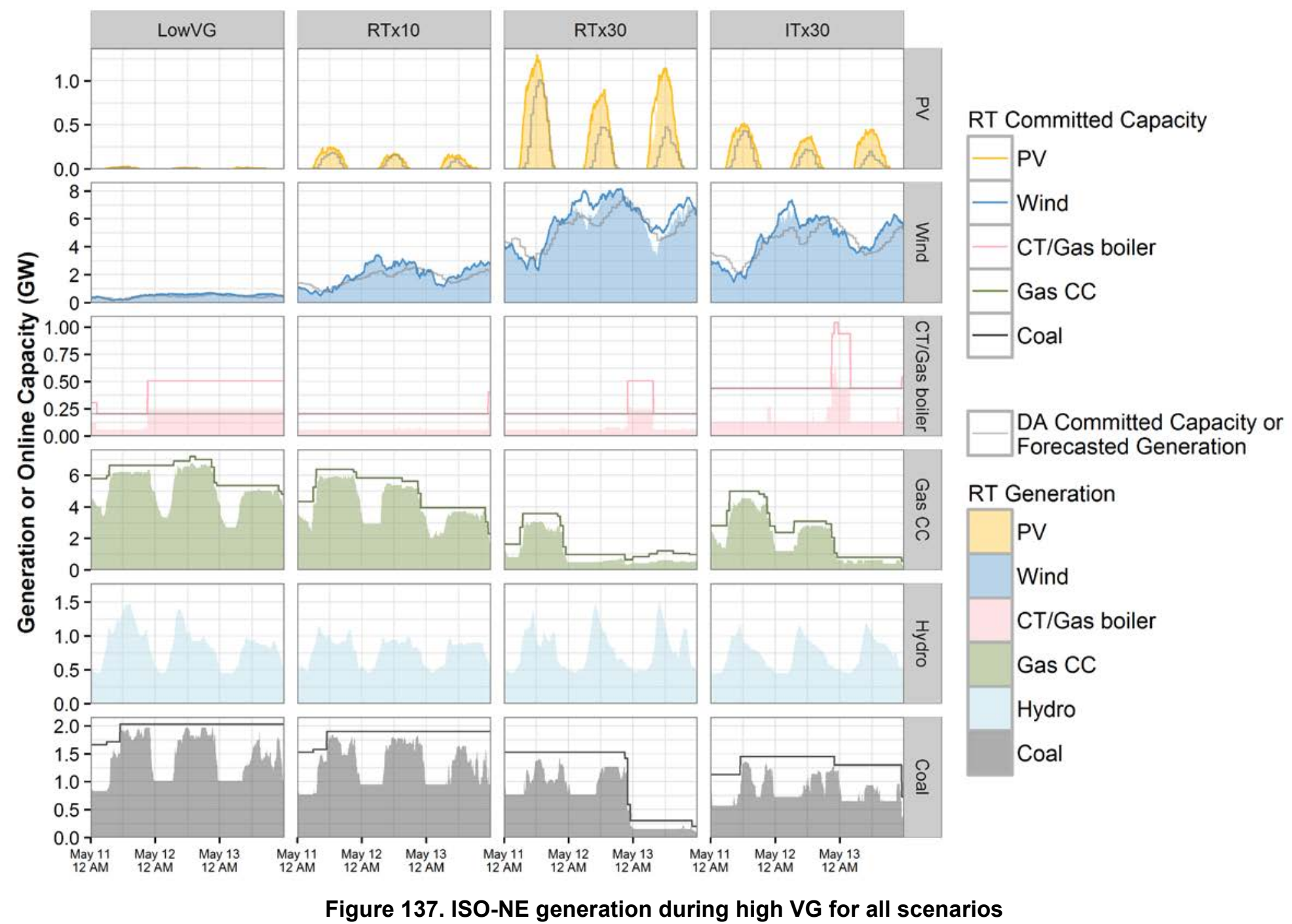




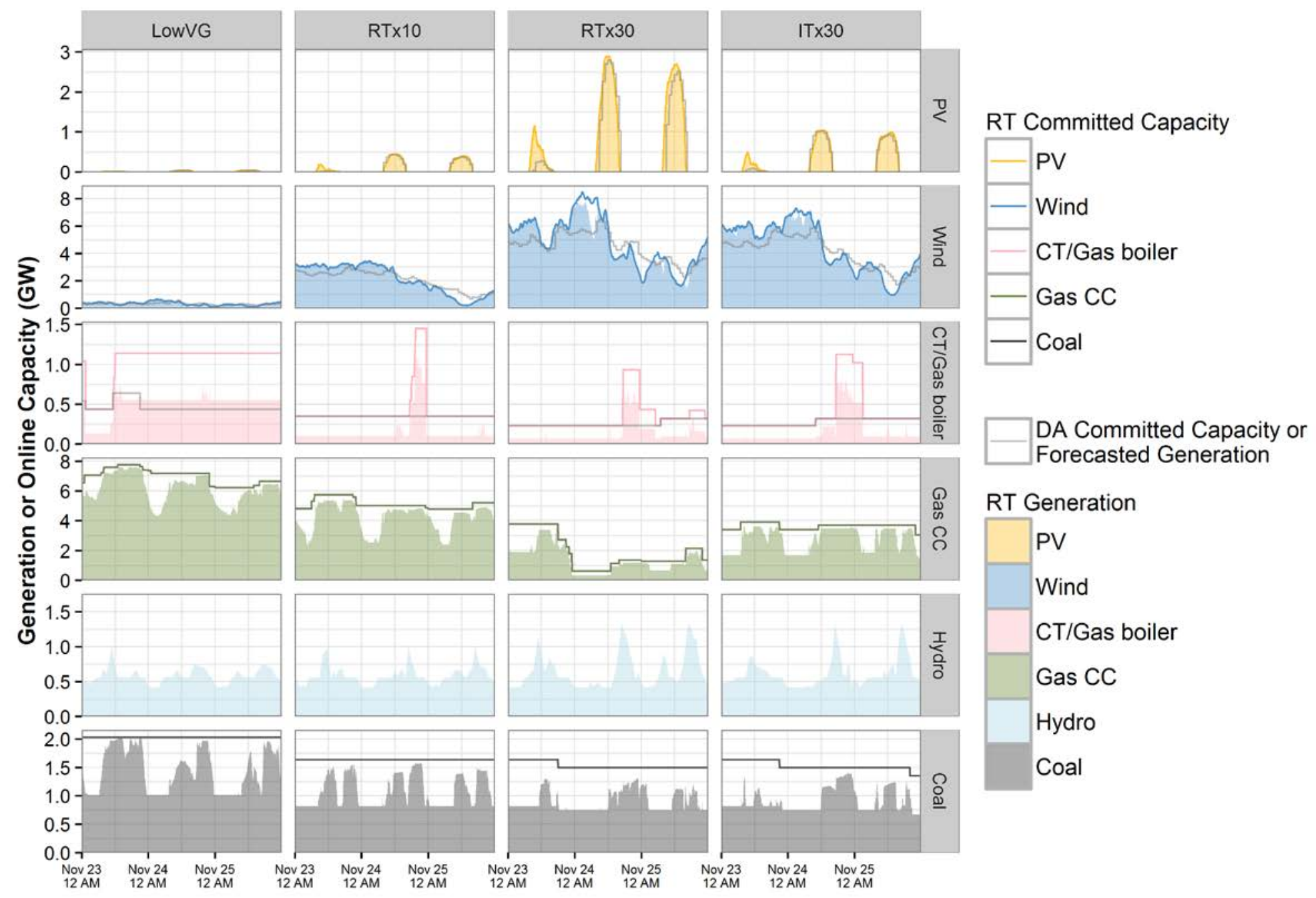

Figure 138. ISO-NE generation during the highest net load ramp for all scenarios 


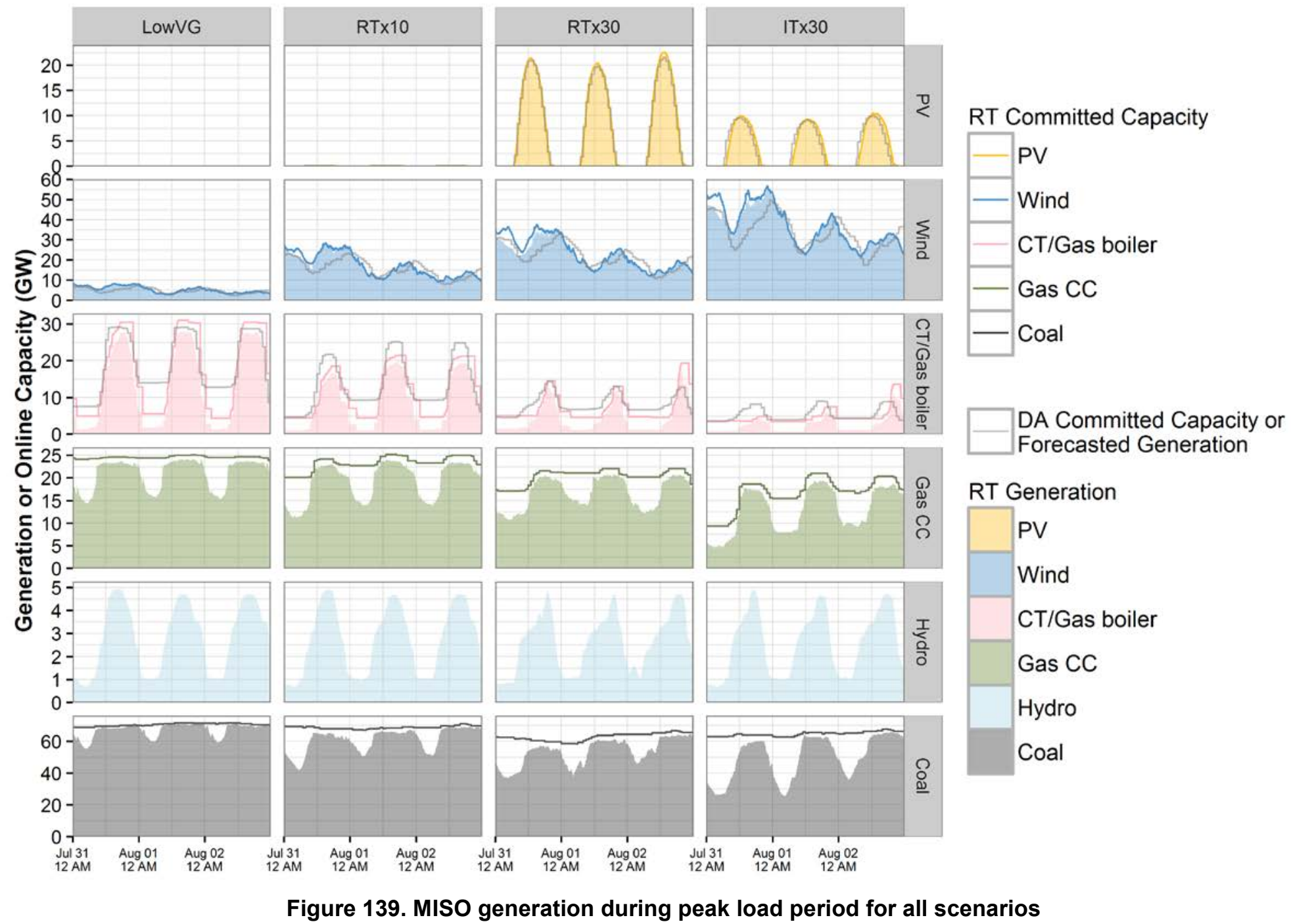




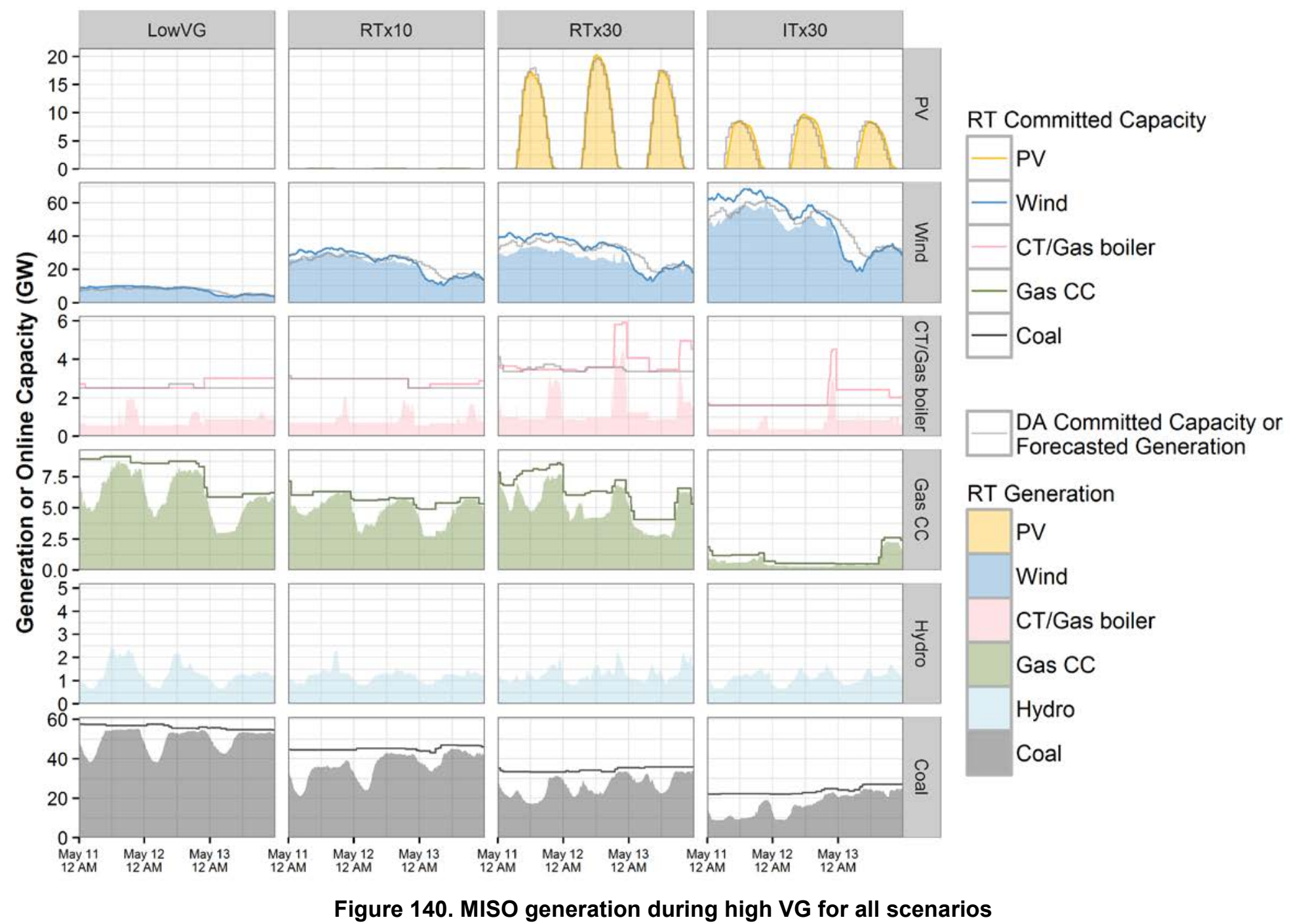




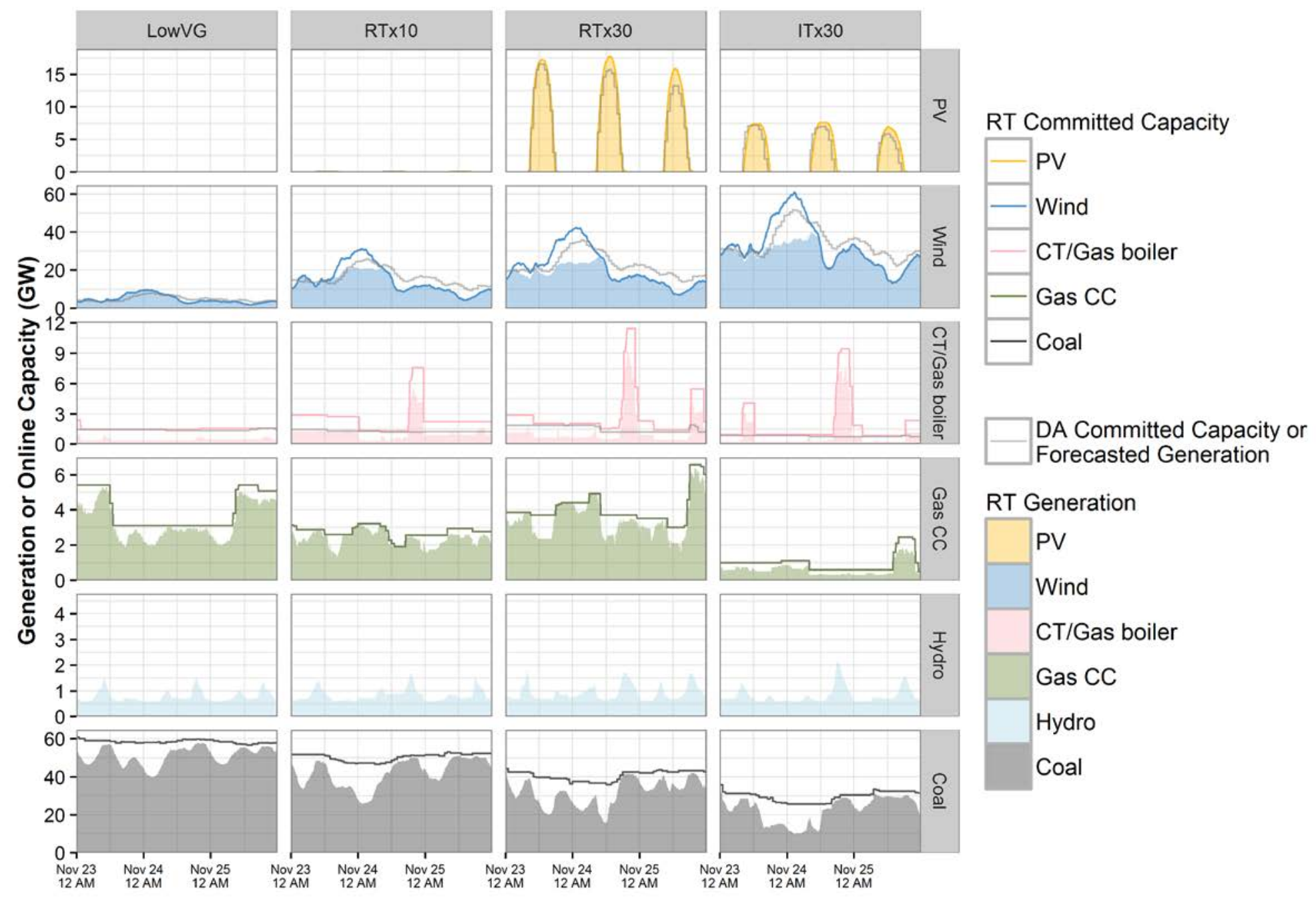

Figure 141. MISO generation during the highest net load ramp for all scenarios 


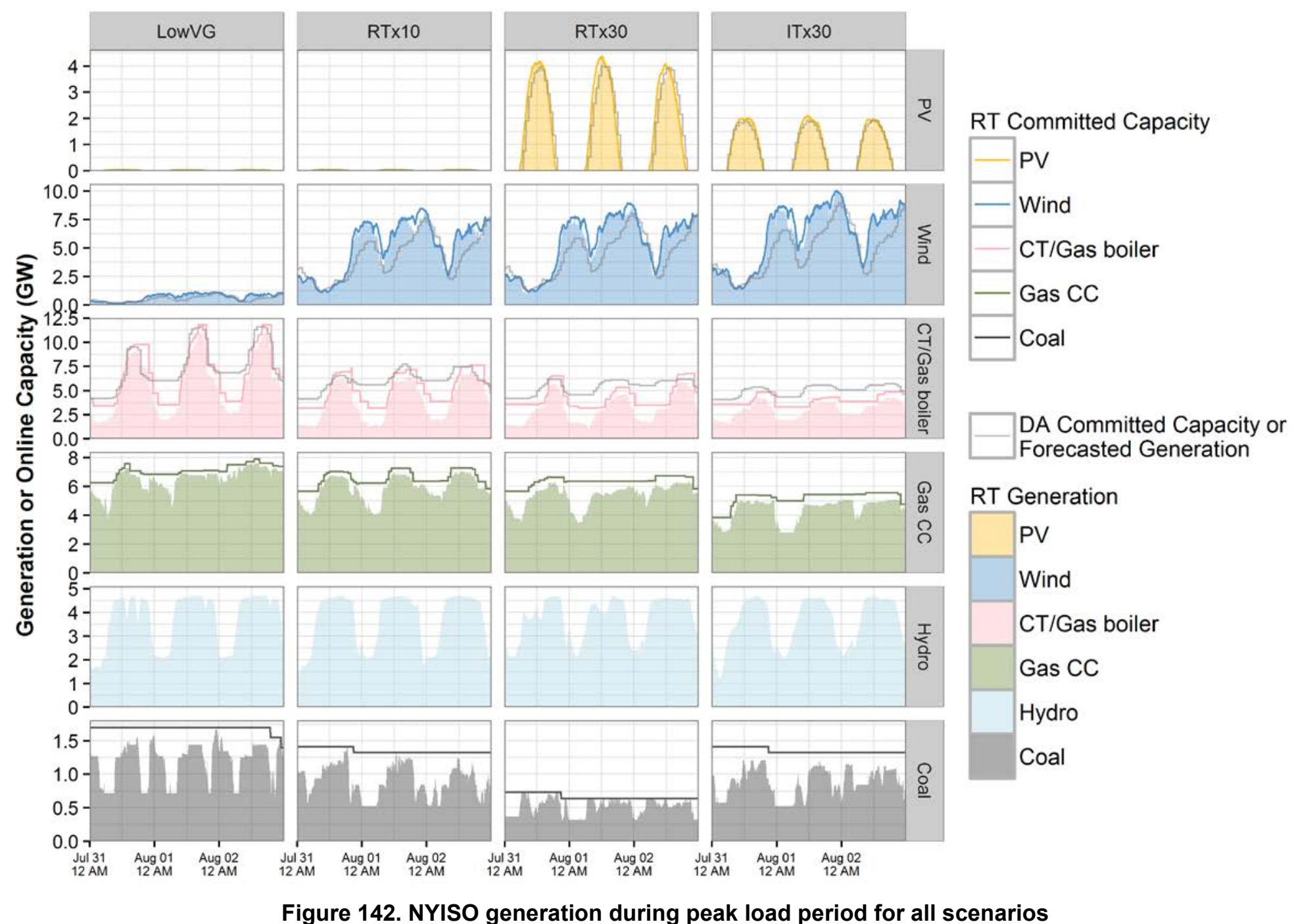




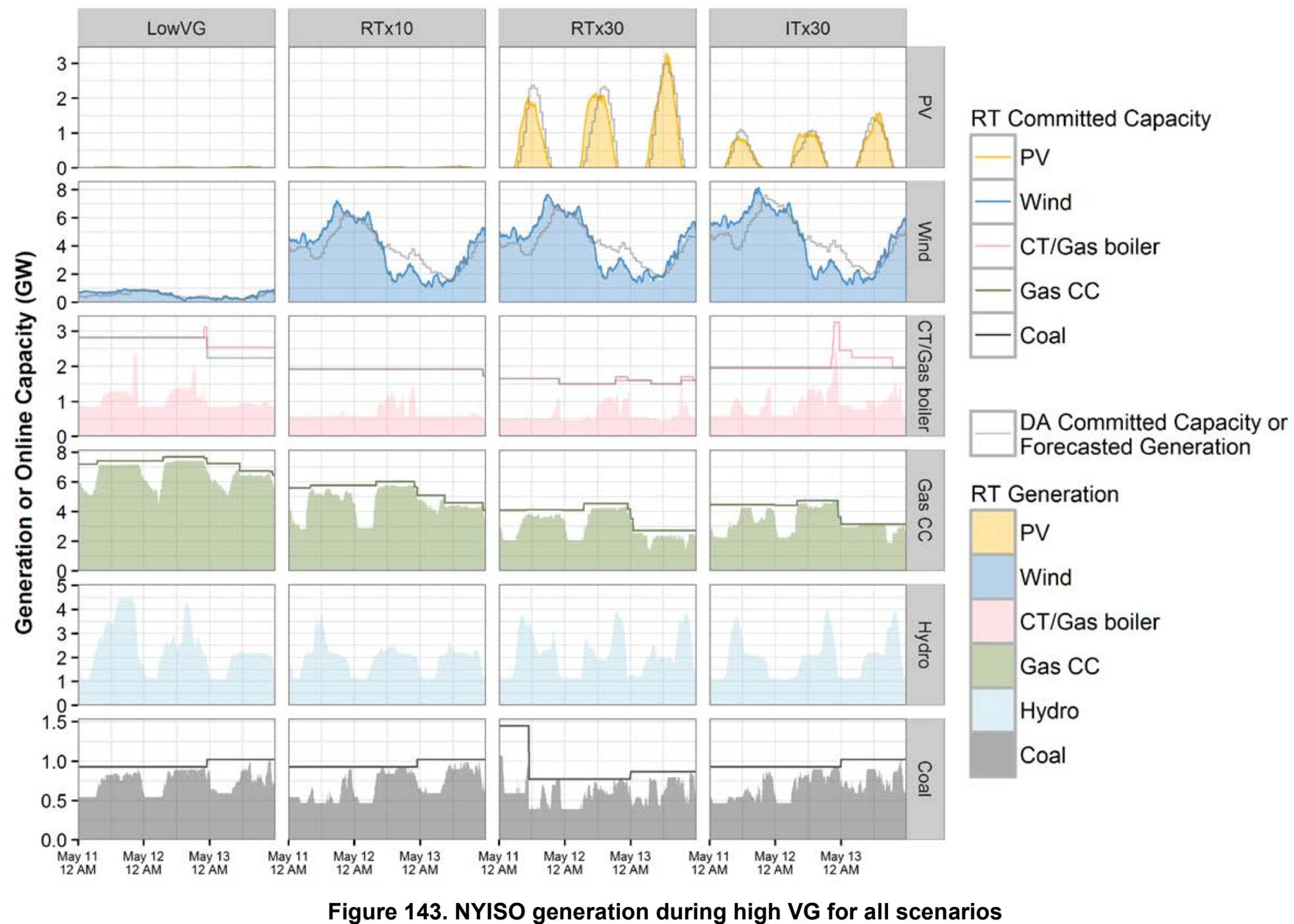




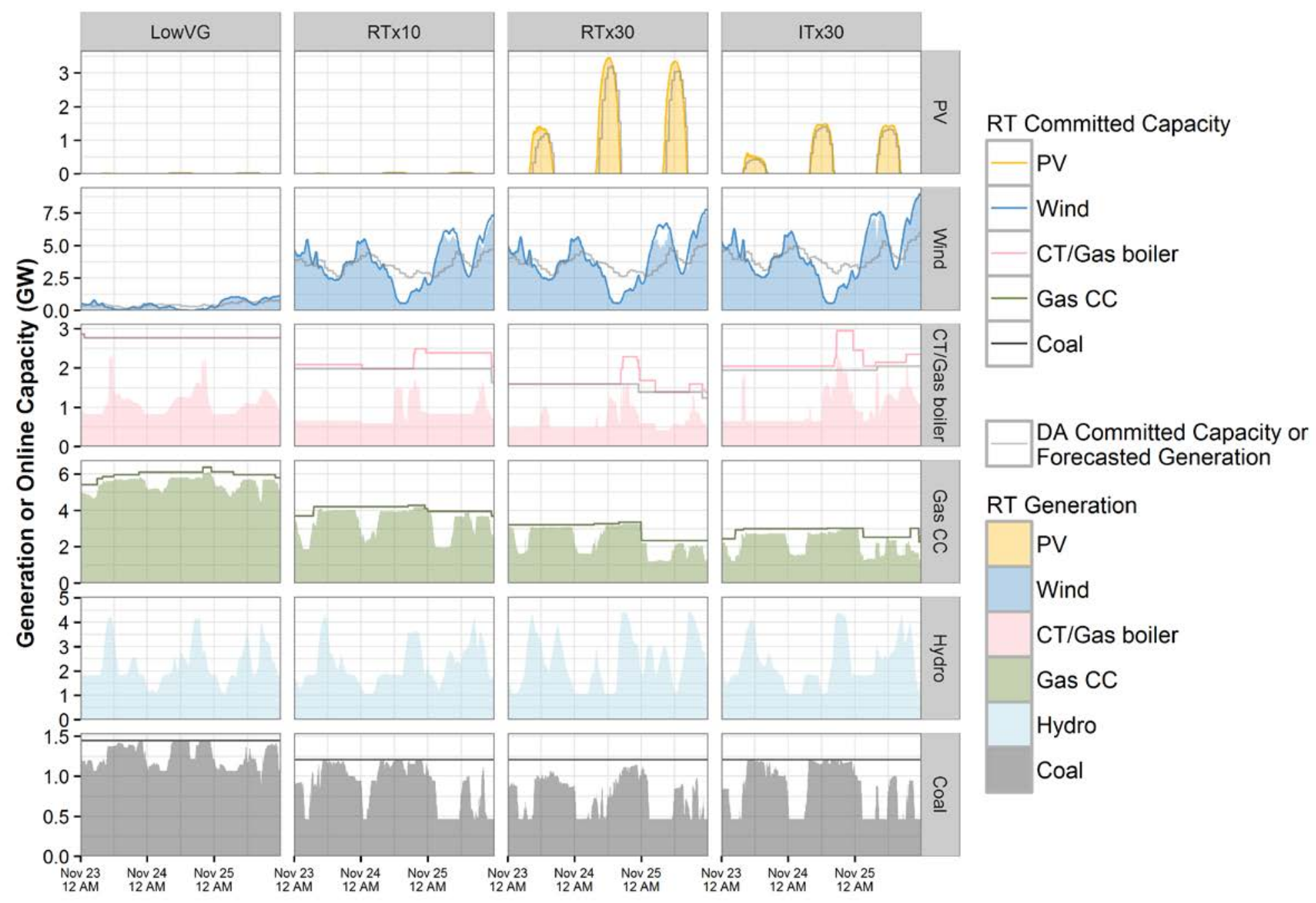

Figure 144. NYISO generation during the highest net load ramp for all scenarios 


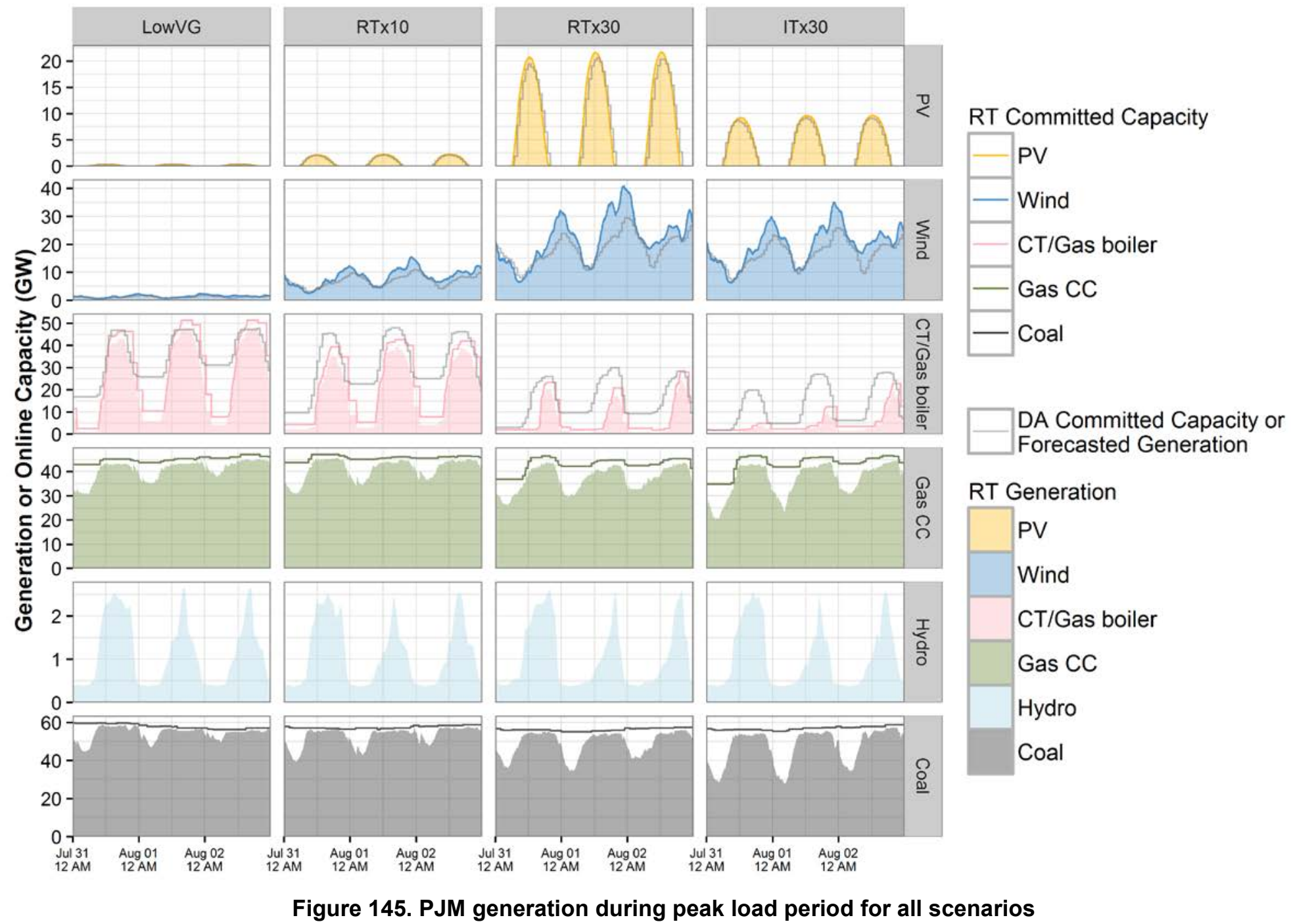




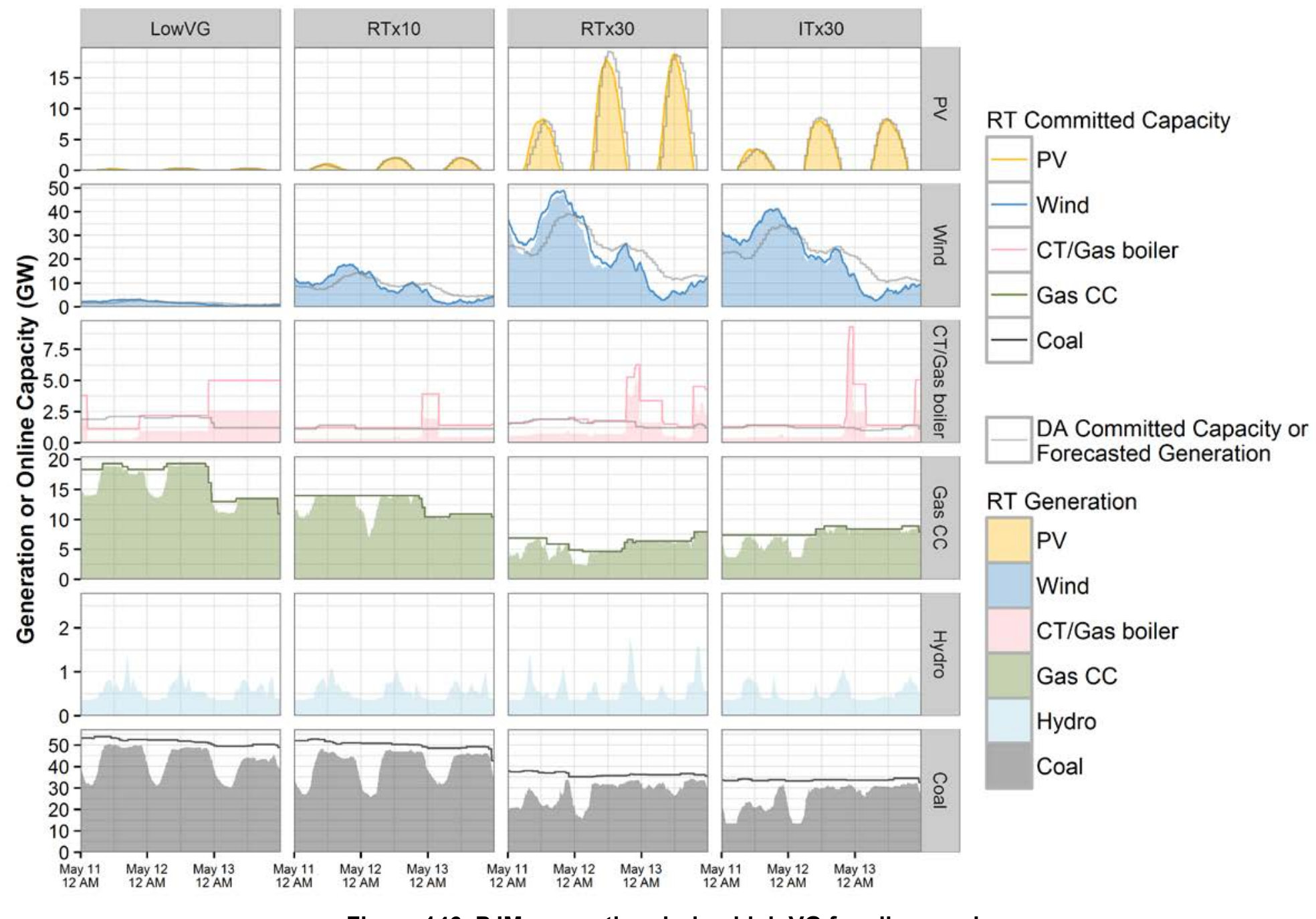

Figure 146. PJM generation during high VG for all scenarios 


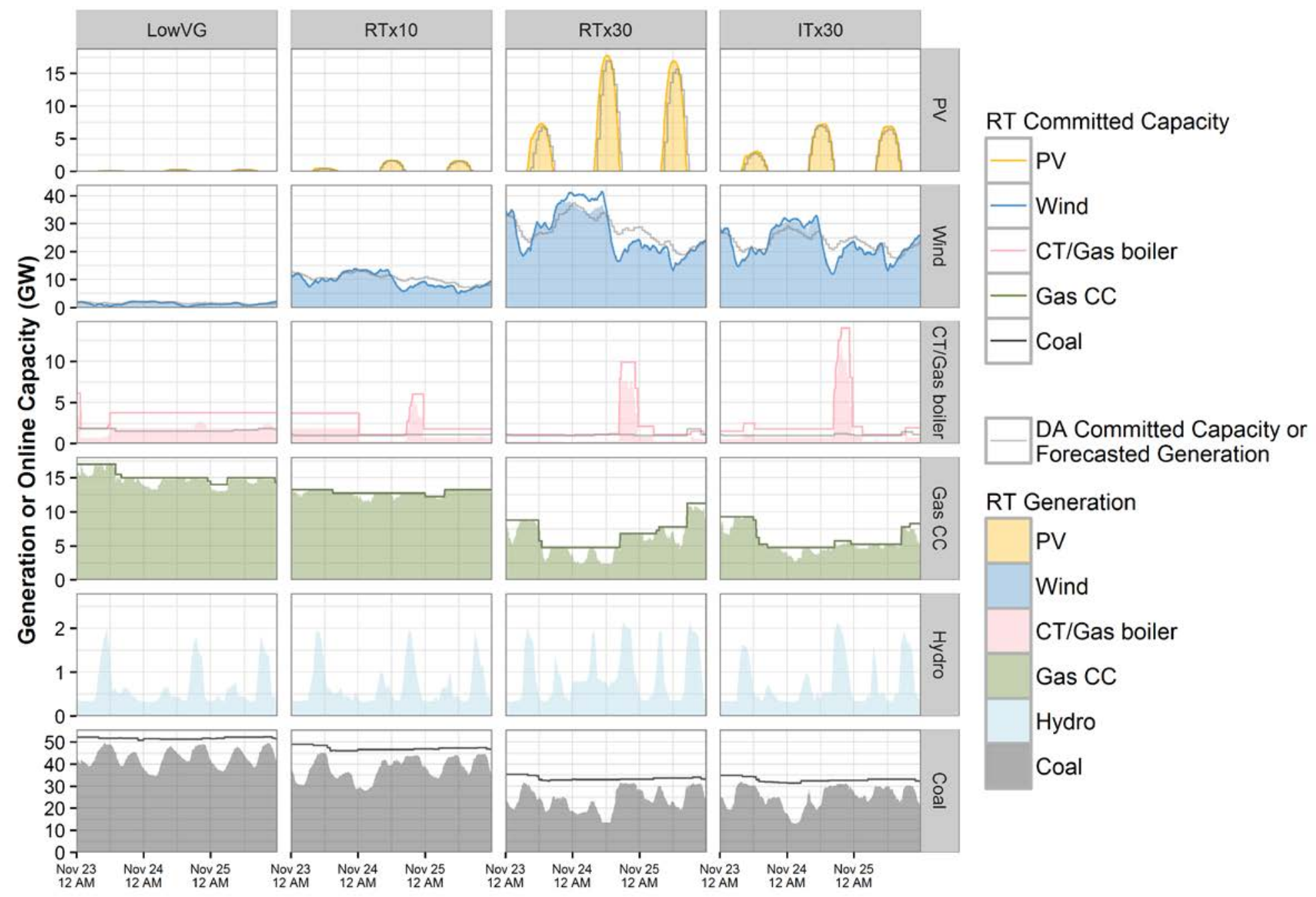

Figure 147. PJM generation during the highest net load ramp for all scenarios 


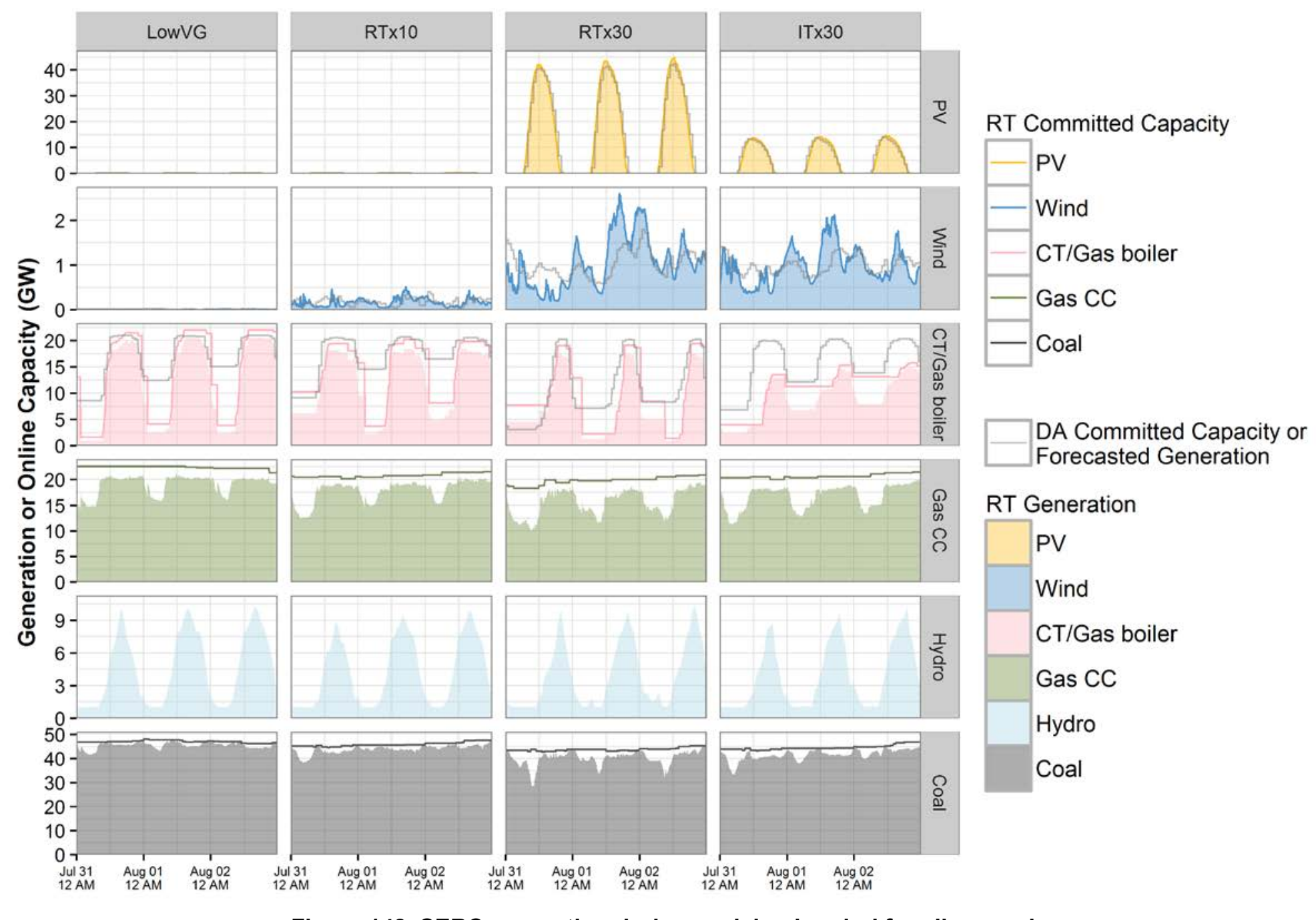

Figure 148. SERC generation during peak load period for all scenarios 


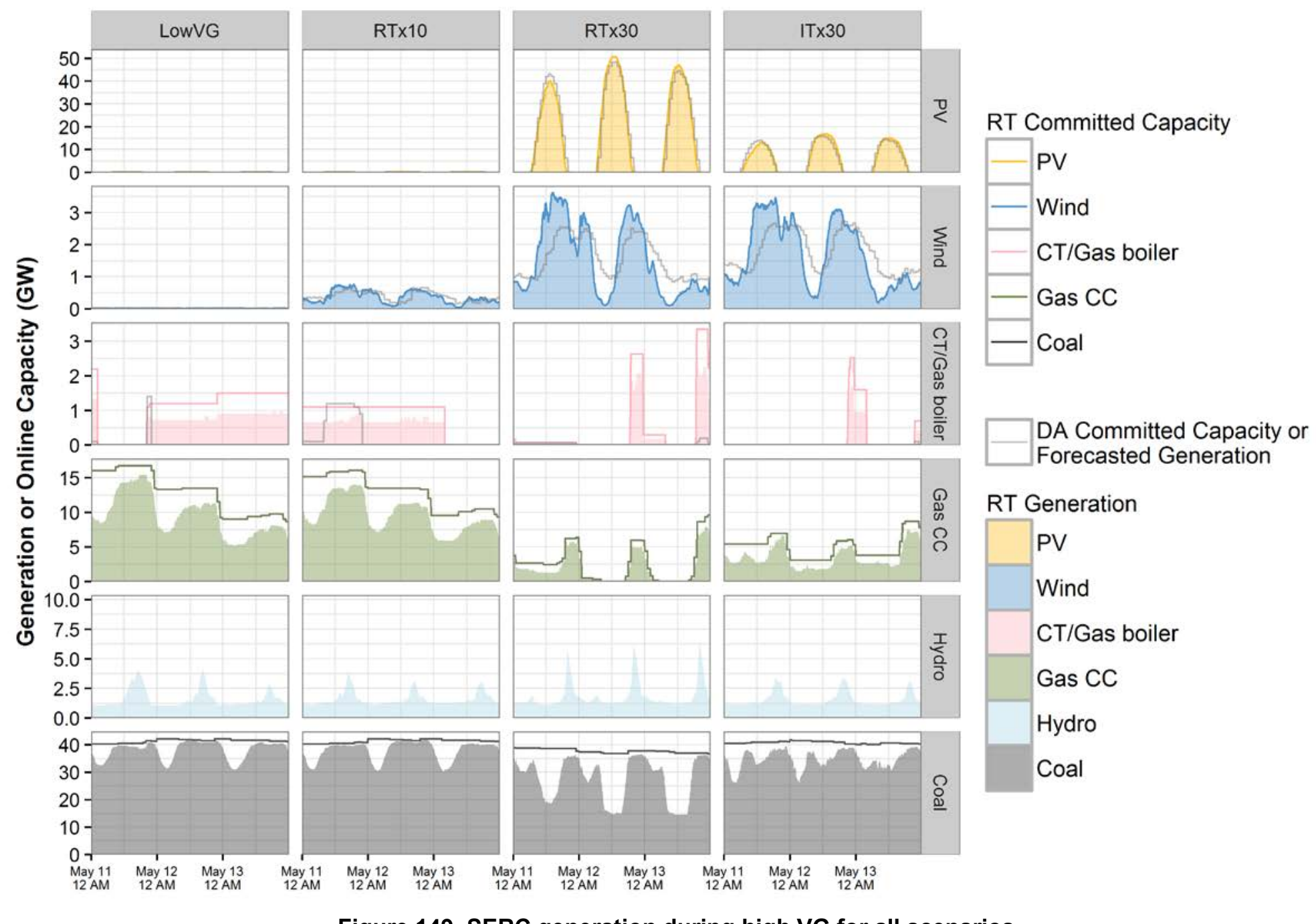

Figure 149. SERC generation during high VG for all scenarios 


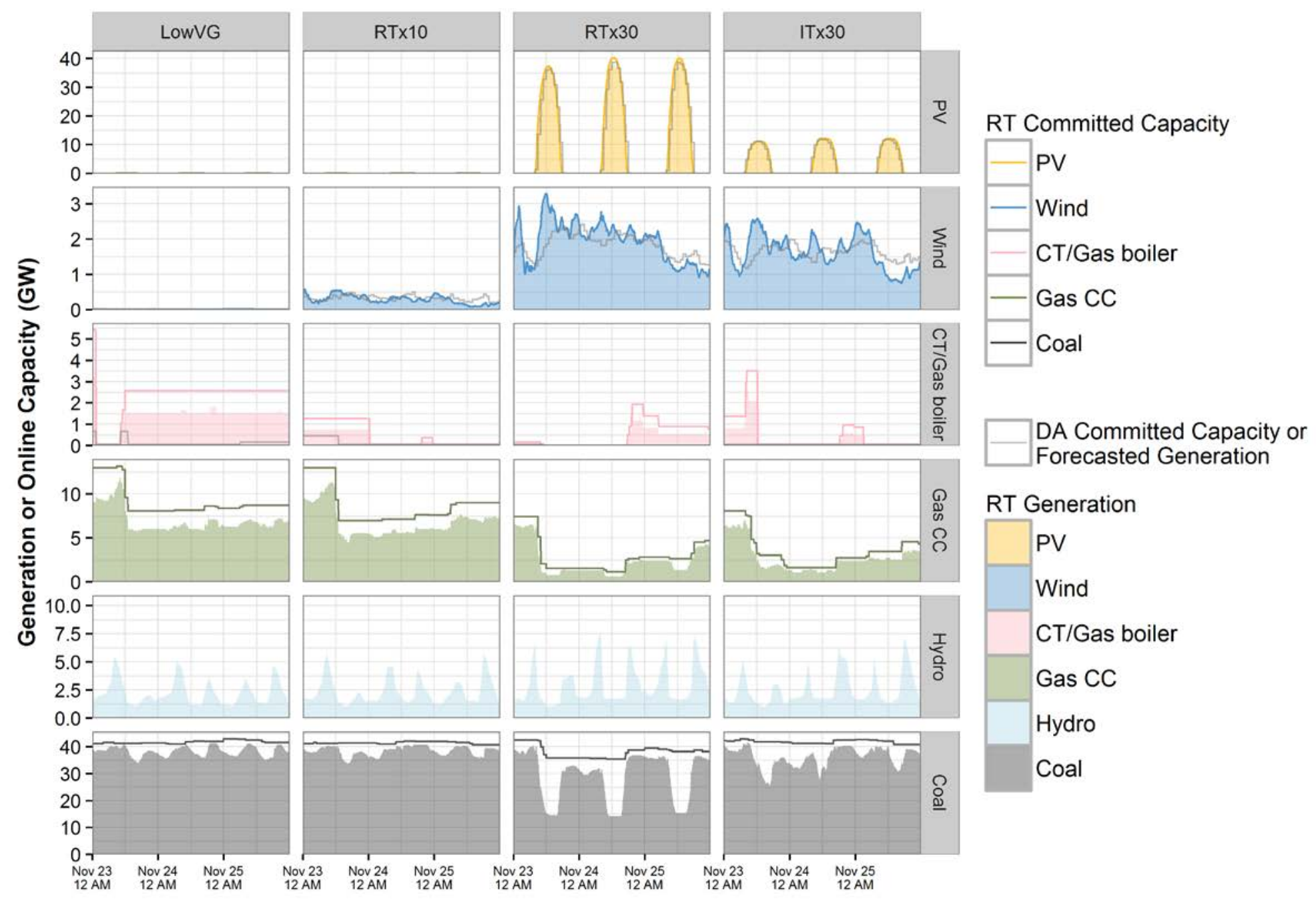

Figure 150. SERC generation during the highest net load ramp for all scenarios 


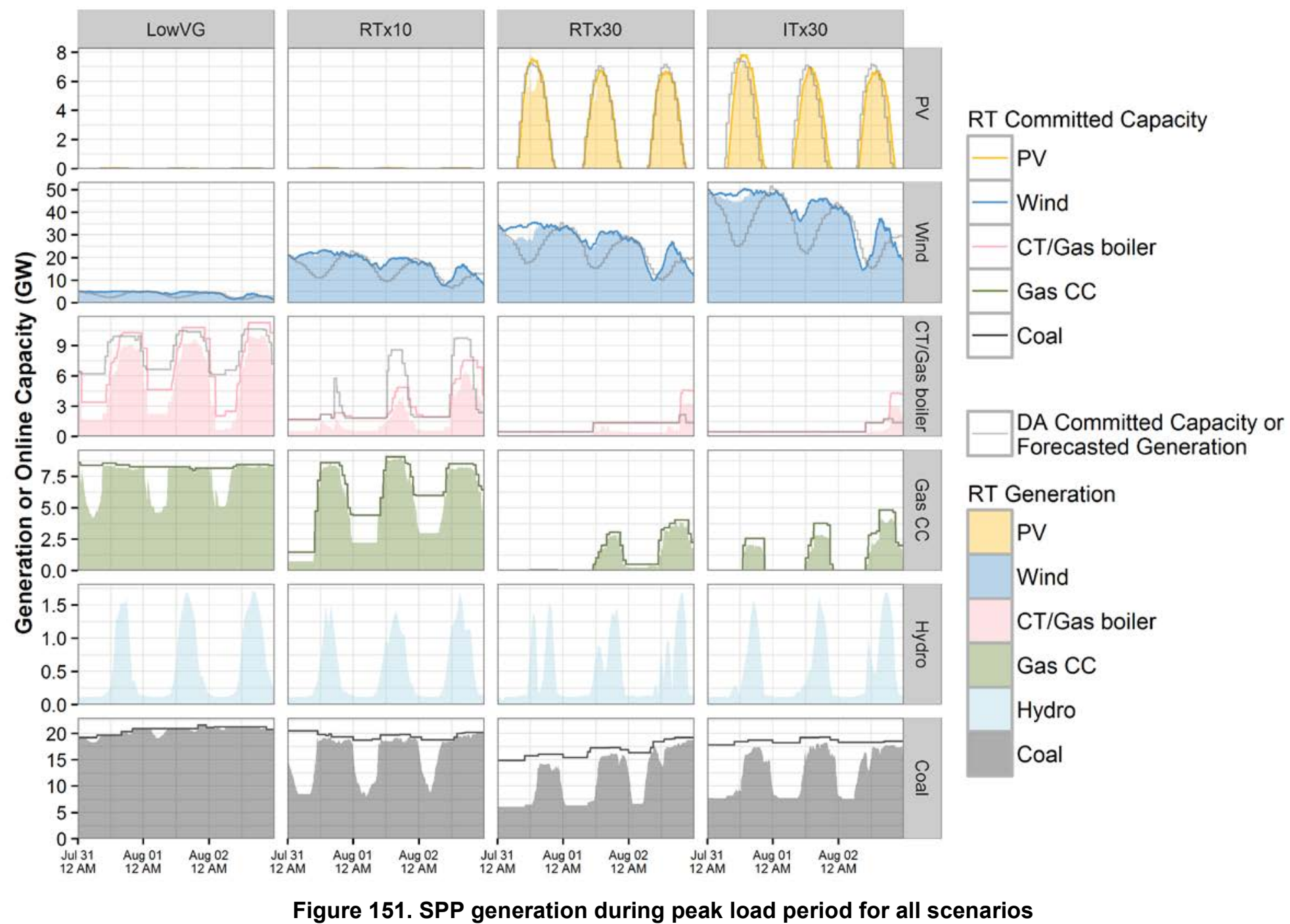




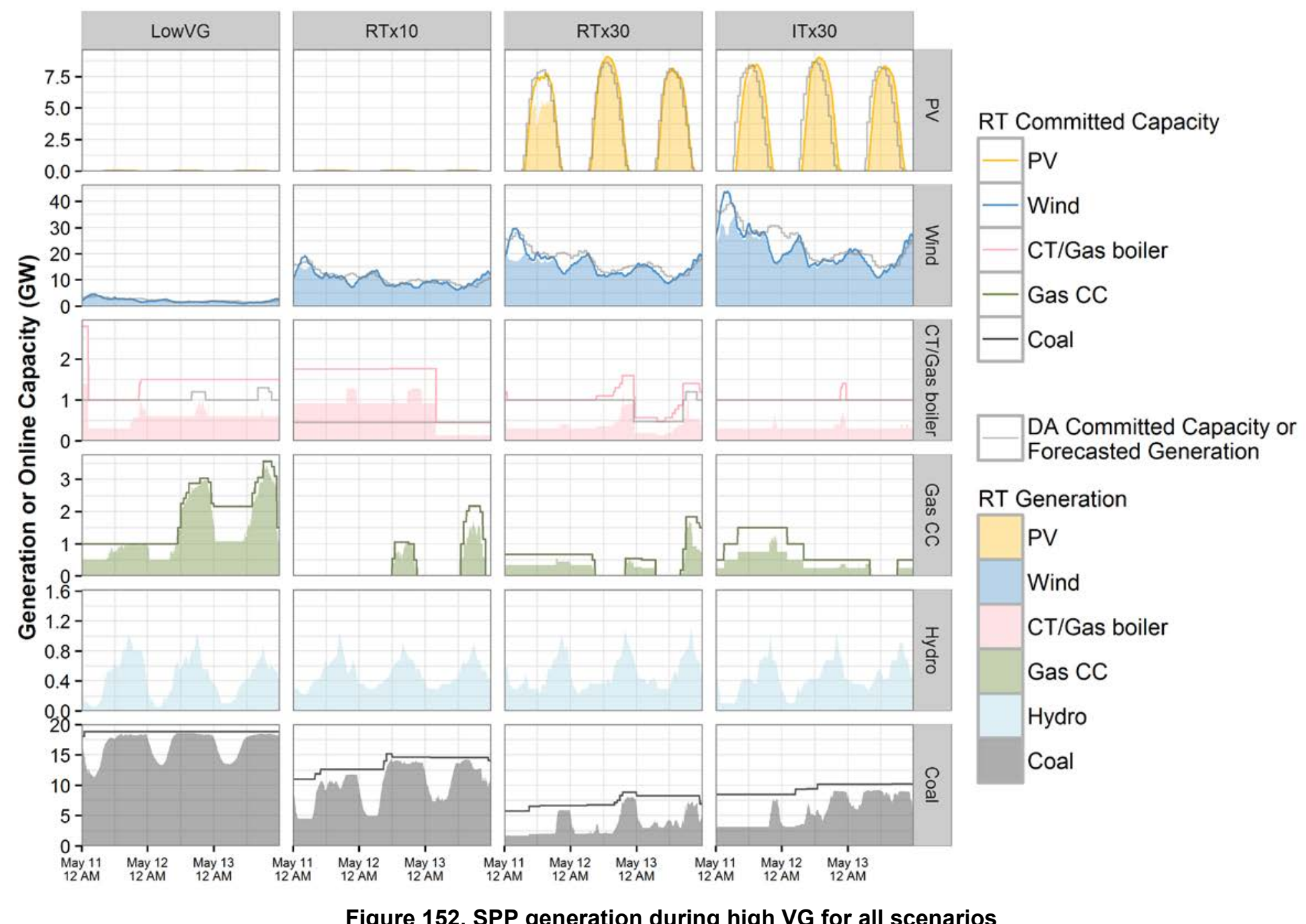

Figure 152. SPP generation during high VG for all scenarios 


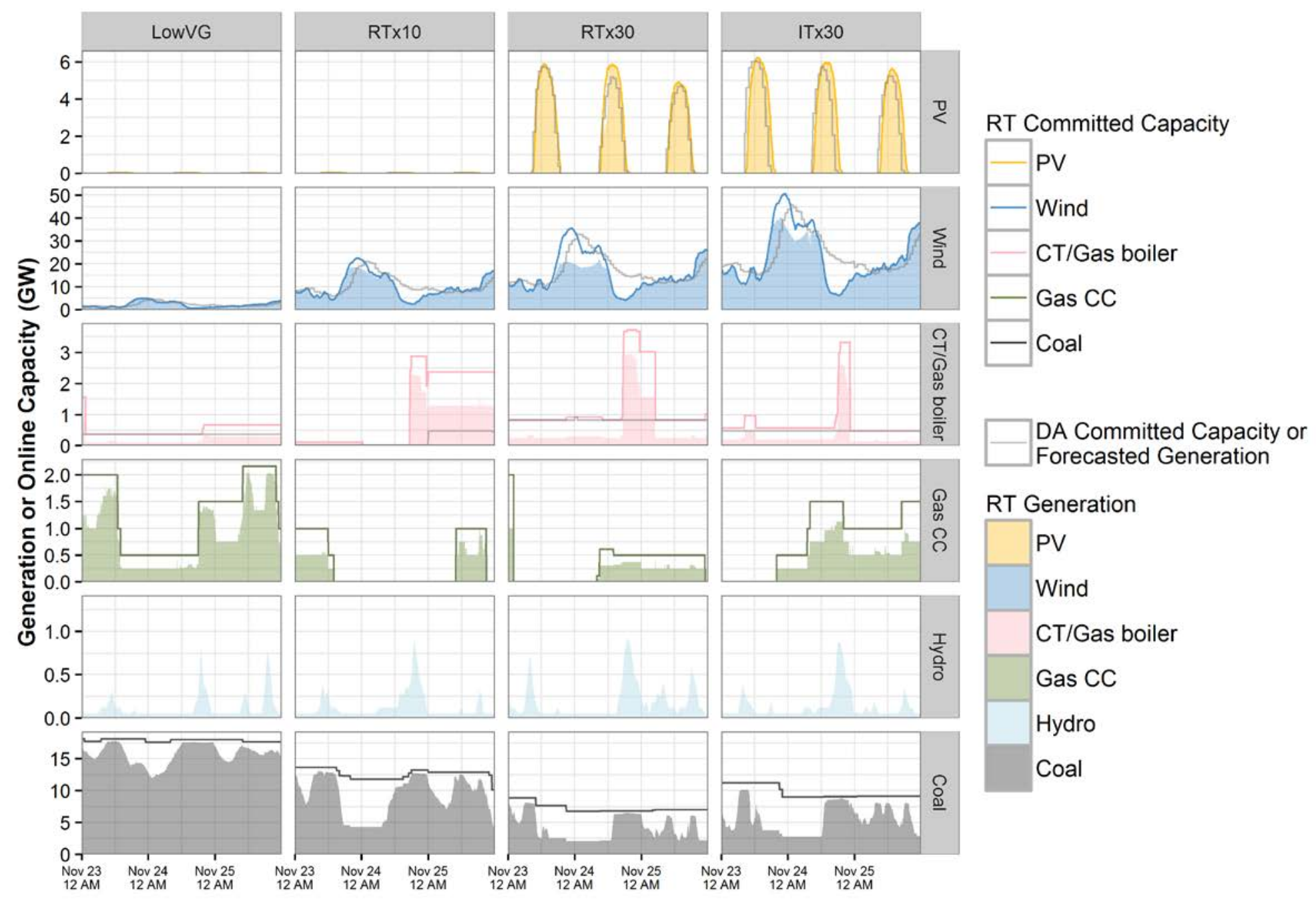

Figure 153. SPP generation during the highest net load ramp for all scenarios 


\section{Appendix F: Total Net Exports}

Table 56. Total net exports (TWh) between ERGIS macro-regions.

\begin{tabular}{|c|c|c|c|c|c|}
\hline Source & Sink & LowVG & RTx10 & RTX30 & ITx30 \\
\hline FRCC & SERC & 4.0 & 3.6 & 4.4 & 3.6 \\
\hline HQ & IESO & 15.4 & 13.2 & 15.1 & 10.2 \\
\hline HQ & ISO-NE & 13.5 & 14.4 & 12.9 & 13.0 \\
\hline HQ & NBSO & 12.5 & 14.7 & 13.0 & 11.5 \\
\hline HQ & NYISO & 9.8 & 7.2 & 7.2 & 15.1 \\
\hline IESO & HQ & -15.4 & -13.2 & -15.1 & -10.2 \\
\hline IESO & Manitoba & 0.4 & -0.2 & -0.6 & -0.3 \\
\hline IESO & MISO & 1.3 & 0.5 & 0.1 & -3.1 \\
\hline IESO & NYISO & 3.7 & -1.1 & -1.1 & -1.2 \\
\hline ISO-NE & $\mathrm{HQ}$ & -13.5 & -14.4 & -12.9 & -13.0 \\
\hline ISO-NE & NBSO & -6.7 & -7.4 & -4.1 & -3.7 \\
\hline ISO-NE & NYISO & -0.6 & -0.8 & -1.4 & -5.1 \\
\hline Manitoba & IESO & -0.4 & 0.2 & 0.6 & 0.3 \\
\hline Manitoba & MISO & 2.1 & -0.4 & -2.2 & 0.6 \\
\hline Manitoba & Saskatchewan & 1.9 & 3.5 & 4.5 & 2.4 \\
\hline MISO & IESO & -1.3 & -0.5 & -0.1 & 3.1 \\
\hline MISO & Manitoba & -2.1 & 0.4 & 2.2 & -0.6 \\
\hline MISO & PJM & 12.0 & 12.2 & 4.8 & 23.7 \\
\hline MISO & Saskatchewan & 0.1 & 0.4 & 0.7 & 0.6 \\
\hline MISO & SERC & 1.9 & 6.5 & 7.9 & 20.6 \\
\hline MISO & SPP & 1.2 & -8.6 & -19.8 & -67.0 \\
\hline NBSO & HQ & -12.5 & -14.7 & -13.0 & -11.5 \\
\hline NBSO & ISO-NE & 6.7 & 7.4 & 4.1 & 3.7 \\
\hline NYISO & HQ & -9.8 & -7.2 & -7.2 & -15.1 \\
\hline NYISO & IESO & -3.7 & 1.1 & 1.1 & 1.2 \\
\hline NYISO & ISO-NE & 0.6 & 0.8 & 1.4 & 5.1 \\
\hline NYISO & PJM & -9.3 & -7.9 & -8.5 & -7.0 \\
\hline PJM & MISO & -12.0 & -12.2 & -4.8 & -23.7 \\
\hline PJM & NYISO & 9.3 & 7.9 & 8.5 & 7.0 \\
\hline PJM & SERC & 15.4 & 17.4 & 10.2 & 16.6 \\
\hline PJM & SPP & NA & NA & NA & -35.8 \\
\hline Saskatchewan & Manitoba & -1.9 & -3.5 & -4.5 & -2.4 \\
\hline Saskatchewan & MISO & -0.1 & -0.4 & -0.7 & -0.6 \\
\hline SERC & FRCC & -4.0 & -3.6 & -4.4 & -3.6 \\
\hline SERC & MISO & -1.9 & -6.5 & -7.9 & -20.6 \\
\hline SERC & PJM & -15.4 & -17.4 & -10.2 & -16.6 \\
\hline SPP & MISO & -1.2 & 8.6 & 19.8 & 67.0 \\
\hline SPP & PJM & NA & NA & NA & 35.8 \\
\hline
\end{tabular}




\section{References}

Bakke, Jordan, Zheng Zhou, and Sumeet Mudgal. n.d. Manitoba Hydro Wind Synergy Study: Final Report. MISO.

Barrows, C.; Hummon, M.; Jones, W.; Hale, E. (2014). Time Domain Partitioning of Electricity Production Cost Simulations. TP-6A20-60969. Golden, CO: National Renewable Energy Laboratory.

Black and Veatch. (2012). "Cost and Performance Data for Power Generation Technologies." Prepared for the National Renewable Energy Laboratory. Accessed May

2015: http://bv.com/docs/reports-studies/nrel-cost-report.pdf.

California ISO, Integration of Renewable Sources: Operational Requirements and Generation Fleet Capacity at 20\% RPS, 2010. https://www.caiso.com/Documents/IntegrationRenewableResources-OperationalRequirementsandGenerationFleetCapabilityAt20PercRPS.pdf

California ISO, What the Duck Curve Tells Us About Managing a Green Grid, 2013. http://www.caiso.com/Documents/FlexibleResourcesHelpRenewables_FastFacts.pdf

CRA International Inc. (2011). “Appendix C: Further Detail Regarding State RPS Modeling by NEEM Region.” Accessed April 30, 2015: http://www.eipconline.com/uploads/2011.01.25_Appendix_C.pdf.

Deane, J.P., G. Drayton, and B.P. Ó Gallachóir. 2014. "The Impact of Sub-Hourly Modelling in Power Systems with Significant Levels of Renewable Generation." Applied Energy 113(2014):152-158.

Denholm, Paul, Joshua Eichman, Tony Markel, and Ookie Ma. 2015. Summary of Market Opportunities for Electric Vehicles and Dispatchable Load in Electrolyzers. Golden, CO: National Renewable Energy Laboratory. http://www.nrel.gov/docs/fy15osti/64172.pdf

Draxl, C.; Clifton, Andrew; Hodge, Bri-Mathias; McCaa, Jim. The Wind Integration National Dataset (WIND) Toolkit, Applied Energy, Volume 151, 1 August 2015, Pages 355-366, ISSN 0306-2619, http://dx.doi.org/10.1016/j.apenergy.2015.03.121.

Eastern Interconnection Planning Collaborative. (2012). "Phase 2 Report: Interregional Transmission Development and Analysis for Three Stakeholder Selected Scenarios.” DOE Award Project DE-OE0000343. Accessed May 2015: http://www.eipconline.com/uploads/20130103_Phase2Report_Part2_Final.pdf.

EirGrid and SONI, Ensuring a Secure, Reliable and Efficient Power System in a Changing Environment, 2011. http://www.eirgrid.com/media/Ensuring_a_Secure_Reliable_and_Efficient_Power_System Report.pdf 
Ela, E.; Milligan, M.; Kirby, B. (2011). Operating Reserves and Variable Generation. NREL/TP-5500-51978. Golden, CO: NREL. Accessed March 2015: http://www.nrel.gov/ docs/fy11osti/51978.pdf.

Ela, E., V. Gevorgian, P. Fleming, Y.C. Zhang, M. Singh, E. Muljadi, A. Scholbrook et al., Active Power Controls from Wind Power: Bridging the Gaps, (Technical Report) NREL/TP5D00-60574, Golden: National Renewable Energy Laboratory, 2014. http://www.nrel.gov/docs/fy14osti/60574.pdf

EnerNex Corporation. (2011). Eastern Wind Integration and Transmission Study. NREL/SR5500-47078. Golden, CO: National Renewable Energy Laboratory. Accessed March 2015: www.nrel.gov/docs/fy11osti/47078.pdf.

EPRI (Electric Power Research Institute). 2011. DOE: Integrating Southwest Power Pool Wind Energy into Southeast Electricity Markets. DE-EE0001377. Palo Alto, CA: EPRI.

EPRI (Electric Power Research Institute). 2014. Program on Technology Innovation: Approach to Transition Nuclear Power Plants to Flexible Power Operations. Palo Alto, CA: EPRI.

Eto, Joseph H., John Undrill, Peter Mackin, Ron Daschmans, Ben Williams, Brian Haney, Randall Hunt et al., Use of Frequency Response Metrics to Assess the Planning and Operating Requirements for Reliable Integration of Variable Renewable Generation, (Technical Report) LBNL-4142E. Berkeley: Lawrence Berkeley National Laboratory, 2010. http://www.ferc.gov/industries/electric/indus-act/reliability/frequencyresponsemetricsreport.pdf

Federal Register. (2014). "Carbon Pollution Emission Guidelines for Existing Stationary Sources: Electric Utility Generating Units."

Generator Availability Data System (accessed April 12, 2013).

http://www.nerc.com/pa/RAPA/gads/Pages/default.aspx

GE Energy. (2010). Western Wind and Solar Integration Study. NREL/SR-550-47434. Golden, CO: National Renewable Energy Laboratory. Accessed March

2015: http://www.nrel.gov/docs/fy10osti/47434.pdf.

GE Energy Consulting. 2014a. PJM Renewable Integration Study Executive Summary Report, Revision 05. GE Energy Consulting. http://www.pjm.com/ /media/committeesgroups/subcommittees/irs/postings/pris-executive-summary.ashx

GE Energy Consulting, 2014b. Minnesota Renewable Energy Integration and Transmission Study, (Final Report). https://mn.gov/commerce/energy/images/final-mrits-report-2014.pdf

Hand, M. M., S. Baldwin, E. DeMeo, J. M. Reilly, T. Mai, D. Arent, G. Porro, M. Meshek, and D. Sandor. (2012) Renewable Electricity Futures Study. Volume 1: Exploration of HighPenetration Renewable Electricity Futures. No. NREL/TP-6A20-52409-1. 
Hummon, M.; Ibanez, E.; Brinkman, G.; Lew, D. (2012). Sub-Hour Solar Data for Power System Modeling From Static Spatial Variability Analysis: Preprint. CP-6A20-56204. Golden, CO: National Renewable Energy Laboratory, 9 pp.

HydroQuébec. (2012.) "Hydroelectric Generating Stations." Accessed [date.] http://www.hydroquebec.com/generation/centrale-hydroelectrique.html.

Ibanez, E.; Milligan, M. (2012). Impact of Transmission on Resource Adequacy in Systems with Wind and Solar Power. IEEE Power\& Energy Society General Meeting, 22-26 July, 2012, San Diego, CA. http://www.nrel.gov/docs/fy12osti/53482.pdf.

Ibanez, E.; Brinkman, G.; Hummon, M.; Lew, D. (2013). "Solar Reserve Methodology for Renewable Energy Integration Studies Based on Sub-hourly Variability Analysis: Preprint." Prepared for the 2nd Annual International Workshop on Integration of Solar Power into Power Systems on November 12-13 in Lisbon, Portugal. NREL/CP-5500-56169. Golden, CO: National Renewable Energy Laboratory, 8 pp. Accessed January 2013: www.nrel.gov/docs/fy12osti/56169.pdf.

Independent Electricity System Operator (IESO). http://www.ieso.ca/imoweb/marketdata/genDisclosure.asp

Jiménez, P.A.; Dudhia, J. (2012). "Improving the Representation of Resolved and Unresolved Topographic Effects on Surface Wind in the WRF Model." J. Appl. Meteor. Climatol., (51); pp. 300-316. Accessed Feb. 26, 2014: http://dx.doi.org/10.1175/JAMC-D-11-084.1

King, J.; Kirby, B.; Milligan, M.; Beuning, S. (2011). Flexibility Reserve Reductions from an Energy Imbalance Market with High Levels of Wind Energy in the Western Interconnection. NREL/TP-5500-52330. Golden, CO: National Renewable Energy Laboratory. Accessed March 2015: http://www.nrel.gov/docs/fy12osti/52330.pdf.

King, J.; Kirby, B.; Milligan, M.; Beuning, S. (May 2012). Operating Reserve Reductions From a Proposed Energy Imbalance Market With Wind and Solar Generation in the Western Interconnection. NREL/TP-5500-54660. Golden, CO: National Renewable Energy Laboratory. Accessed May 27, 2015: http://www.nrel.gov/docs/fy12osti/54660.pdf.

Krzywinski, Martin, Jacqueline Schein, İnanç Birol, Joseph Connors, Randy Gascoyne, Doug Horsman, Steven J. Jones, and Marco A. Marra. 2009. "Circos: An Information Aesthetic for Comparative Genomics." Genome Res. 19: 1639-1645.

Kumar, N.; Besuner, P.; Lefton, S.; Agan, D.; Hilleman, D. (2012). Power Plant Cycling Costs. AES 12047831-2-1. Louisville, CO: Intertek, APTECH.

Lew, D.; Brinkman, G.; Kumar, N.; Besuner, P.; Agan, D.; Lefton, S. "Impacts of Wind and Solar on Fossil-Fueled Generators," Tech. Rep. August, National Renewable Energy Laboratory, Golden, CO, 2012. 
Lew, D.; Brinkman, G.; Ibanez, E.; Florita, A.; Heaney, M.; Hodge, B. M.; Hummon, M.; Stark, G.; King, J.; Lefton, S. A.; Kumar, N.; Agan, D.; Jordan, G.; Venkataraman, S. (2013). Western Wind and Solar Integration Study Phase 2. NREL/TP-5500-55588. Golden, CO: National Renewable Energy Laboratory. Accessed March

2015: http://www.nrel.gov/docs/fy13osti/55588.pdf.

Manitoba Hydro. (2014). Re-Powering Our Province: Annual Report 2013-14." Accessed [date.] http://www.hydro.mb.ca/corporate/ar/index.shtml

Miller, Nicholas W., Miaolei Shao, and Sundar Venkataraman, California ISO (CAISO)

Frequency Response Study (Report), GE Energy Consulting,

2011. http://www.caiso.com/Documents/Report-FrequencyResponseStudy.pdf

Miller, N.W., M. Shao, S. Pajic, and R. D'Aquila, Western Wind and Solar Integration Study Phase 3 -Frequency Response and Transient Stability, (Subcontract Report) NREL/SR-5D0062906. Golden: National Renewable Energy Laboratory,

2014. http://www.nrel.gov/docs/fy15osti/62906.pdf

Mills, A., Wiser, R. (2010). Implications of wide-area geographic diversity for short-term variability of solar power, Lawerence Berkeley National Laboratory, Tech. Rep. LBNL-3884E, 2010 .

National Renewable Energy Laboratory. (2012). Renewable Electricity Futures Study. Hand, M.M.; Baldwin, S.; DeMeo, E.; Reilly, J.M.; Mai, T.; Arent, D.; Porro, G.; Meshek, M.; Sandor, D. eds. 4 vols. NREL/TP-6A20-52409. Golden, CO: National Renewable Energy

Laboratory. http://www.nrel.gov/analysis/re_futures/.

National Renewable Energy Laboratory. (2015). High Performance Computing. Accessed June 1, 2015. https://hpc.nrel.gov/systems

Pennock, K. (2012). Updated Eastern Interconnection Wind Power Output and Forecasts for ERGIS. NREL/SR-5500-56616. Golden, CO: National Renewable Energy Laboratory.

PJM (2016). PJM Manual 12: Balancing Operations. (Revision 34).

http://www.pjm.com/ /media/documents/manuals/m12.ashx

PLEXOS Integrated Energy Model version 6.400 R02 x64 Edition [computer software]. (2014). Accessed May 20, 2015: http://energyexemplar.com/.

Saha, A. (2013). "Review of Coal Retirements." Concord, MA: MJ Bradley and Associates.

SaskPower. (2012). "SaskPower Annual Report 2012." http://www.saskpower.com/wpcontent/uploads/2012_saskpower_annual_report.pdf

Short, W.; Sullivan, P.; Mai, T.; Mowers, M.; Uriarte, C.; Blair, N.; Heimiller, D.; Martinez, A. (2011). Regional Energy Deployment System (ReEDS). TP-6A20-46534. Golden, CO: National Renewable Energy Laboratory, 94 pp. 
Skamarock, W.C.; Klemp, J.B.; Dudhia, J.; Gill, D.O.; Barker, D.M.; Duda, M.G.; Huang, X.Y.; Wang, W.; Powers, J.G. (2008). A description of the Advanced Research WRF Version 3. NCAR Tech Notes-475+STR. Boulder, CO: National Center for Atmospheric Research.

Stoll, Brady, Gregory Brinkman, Aaron Townsend, and Aaron Bloom. 2016. Analysis of Modeling Assumptions used in Production Cost Models for Renewable Integration Studies. Golden, CO: National Renewable Energy Laboratory.

Tegen, I.; Hollrig, P.; Chin, M.; Fung, I.; Jacob, D.; Penner, J. (1997). "Contribution of Different Aerosol Species to the Global Aerosol Extinction Optical Thickness: Estimates From Model Results." J. Geophys. Res.(102); pp. 23895-23915. doi:10.1029/97JD01864.

Tennessee Valley Authority. 2015. "TVA Reservoirs and Power Plants" (Interactive map.) Accessed [date]. http://www.tva.gov/sites/sites ie.htm.

U.S. Army Corps of Engineers Mobile District, Water Management Section. 2015a. ACF (Apalachicola-Chattahoochee-Flint) Historic Project Data." U.S. Army Corps of Engineers. Accessed [month/day, include year if not give after "author".] http://water.sam.usace.army.mil/gage/acfhist.htm.

U.S. Army Corps of Engineers Mobile District, Water Management Section. 2015b. "ACT (Alabama-Coosa-Tallapoosa) Historic Project Data." U.S. Army Corps of Engineers. Accessed [date.] http://water.sam.usace.army.mil/gage/acthist.htm.

U.S. Department of Energy (DOE) Office of Energy Efficiency and Renewable Energy. (2008). "20\% Wind Energy by 2030: Increasing Wind Energy Contribution to the U.S. Electricity Supply.” DOE/GO-102008-2567. Washington, DC: DOE.

U.S. Department of Energy (DOE) Office of Energy Efficiency and Renewable Energy. (2012). "SunShot Vision Study." DOE/GO-102012-3037. Washington, DC: DOE.

U.S. Department of Energy. (2015). "WindVision: A New Era for Wind Power in the United States." DOE/GO-102015-4557. Washington, DC: DOE.

U.S. Energy Information Administration. (2011a). "The Electricity Market Module of the National Energy Modeling System Model Documentation Report." Office of Integrated Analysis and Forecasting. July

2011. http://www.eia.gov/forecasts/aeo/nems/documentation/electricity/pdf/m068\%282011\%29. pdf

U.S. Energy Information Administration. (2011b). "Today in Energy: Electricity Tends to Flow South in North America." http://www.eia.gov/todayinenergy/detail.cfm?id=4270.

U.S. Energy Information Administration. (2013). “Annual Energy Outlook 2014 Early Release.” Washington, DC: U.S. Energy Information Administration. 
U.S. Energy Information Administration. (2015a). "Electric Power Annual 2013." Washington, DC: U.S. Energy Information Administration. http://www.eia.gov/electricity/data/eia923/U.S. Energy Information Administration. (2015b). "Retail Sales of Electricity by State by Sector by Provider (EIA-861)." April, 2005. http://www.eia.gov/electricity/data/state/.

U.S. Energy Information Administration. (2015c) "Electricity: Form EIA=923 Detailed Data." http://www.eia.gov/electricity/data/eia923/

Ontario Power Generation. (2015). "River System Data." http://www.opg.com/generating-power/hydro

ABB Energy. (2013). Velocity Suite. Data Product. Boulder, CO: ABB Energy.

Zhang, Y.C., V. Gevorgian, E. Ela, V. Singhvi, and P. Pourbeik, "Role of Wind Power in Primary Frequency Response of an Interconnection: Preprint," Paper presented at the International Workshop on Large-Scale Integration of Wind Power into Power Systems as Well as on Transmission Networks for Offshore Wind Power Plants, London, United Kingdom, October 22-24, 2013. NREL/CP-5D00-58995. http://www.nrel.gov/docs/fy13osti/58995.pdf 